\title{
Modeling and Controlling a Hybrid Multi-Agent based Microgrid in Presence of Different Physical and Cyber Components
}

\author{
Hessam Keshtkar
}

Follow this and additional works at: https://researchrepository.wvu.edu/etd

\footnotetext{
Recommended Citation

Keshtkar, Hessam, "Modeling and Controlling a Hybrid Multi-Agent based Microgrid in Presence of Different Physical and Cyber Components" (2015). Graduate Theses, Dissertations, and Problem Reports. 7320.

https://researchrepository.wvu.edu/etd/7320

This Dissertation is protected by copyright and/or related rights. It has been brought to you by the The Research Repository @ WVU with permission from the rights-holder(s). You are free to use this Dissertation in any way that is permitted by the copyright and related rights legislation that applies to your use. For other uses you must obtain permission from the rights-holder(s) directly, unless additional rights are indicated by a Creative Commons license in the record and/ or on the work itself. This Dissertation has been accepted for inclusion in WVU Graduate Theses, Dissertations, and Problem Reports collection by an authorized administrator of The Research Repository @ WVU. For more information, please contact researchrepository@mail.wvu.edu.
} 


\title{
Modeling and Controlling a Hybrid Multi-Agent based Microgrid in Presence of Different Physical and Cyber Components
}

\author{
Hessam Keshtkar \\ Dissertation submitted to the \\ Benjamin M. Statler College of Engineering and Mineral Resources \\ at West Virginia University \\ in partial fulfillment of the requirements for the degree of \\ Doctor of Philosophy in \\ Electrical Engineering \\ Dr. Jignesh Solanki, Chair. \\ Dr. Sarika Khushalani Solanki. \\ Dr. Ali Feliachi. \\ Dr. Afzal Noore. \\ Dr. Rong Luo.
}

Lane Department of Computer Science and Electrical Engineering

Morgantown, West Virginia

2015

Keywords: Impedance Calculation, Microgrid Control, Multi-Agent System, Hierarchical Structure, Adaptive Dynamic Programming, Stochastic Uncertainties, Cyber Intrusions 
ProQuest Number: 10110042

All rights reserved

INFORMATION TO ALL USERS

The quality of this reproduction is dependent upon the quality of the copy submitted.

In the unlikely event that the author did not send a complete manuscript and there are missing pages, these will be noted. Also, if material had to be removed, a note will indicate the deletion.

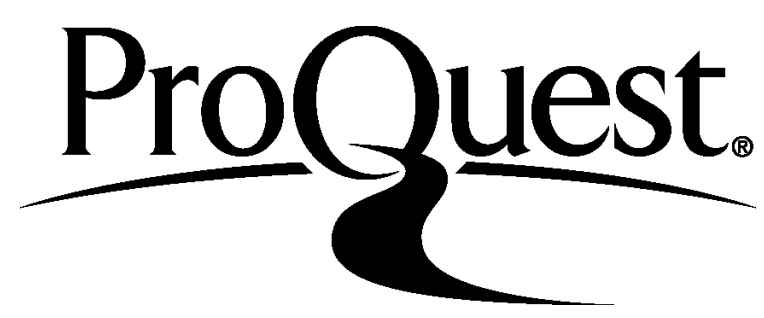

ProQuest 10110042

Published by ProQuest LLC (2016). Copyright of the Dissertation is held by the Author.

All rights reserved.

This work is protected against unauthorized copying under Title 17, United States Code Microform Edition (c) ProQuest LLC.

ProQuest LLC.

789 East Eisenhower Parkway

P.O. Box 1346

Ann Arbor, Ml 48106 - 1346 


\title{
Abstract \\ Modeling and Controlling a Hybrid Multi-Agent based Microgrid in Presence of Different Physical and Cyber Components
}

\author{
by \\ Hessam Keshtkar
}

\begin{abstract}
Modeling a power system and its components is very critical and first step to perform important analysis on the system, especially with penetration of Distributed Generation (DG) units. For instance, calculation of the line parameters or the location of DG units is very important for analyzing a distribution power system. The impedances are used for modeling underground/ overhead lines and cables of a distribution system whose accuracy defines the accuracy of unbalanced distribution power flow solutions. The complexities of the exact representation of the distribution line parameters have led investigators to propose approximate formulae. The accuracy of these approximation methods affects the results of fault analysis and designing aspects of protection devices such as fault clearing time of circuit breakers in a three-phase unbalanced distribution system. Therefore, investigation on accurate modeling of the distribution line impedances seems necessary. Also, depending on the location and steady state modeling of DG units, the results of the power flow analysis will be changed which results in different total line loss in the system. Hence it is important to model and site the DG units to optimally exploit their potential. One of the DG technologies is Photovoltaic (PV), with penetrations increasing from hundreds of kWs to MWs in LV network. Due to these increasing penetrations in distribution systems, the utilities and planning engineers are increasingly interested in determining the best model and locations to place these units.

The distribution power systems having small scale energy sources such as DGs, Renewable Energy Sources (RES), and Plug-in Hybrid Electric Vehicles (PHEVs) are called as microgrids. Modeling and controlling microgrids bring forth numerous challenges to system operation and design. The main aim of microgrid operation control is to reliably serve the loads while maintaining the security and stability of the system. Oscillatory stability, especially during rapid changes in load and generation, has become of the most important problems lately due to integration of intermittent renewable sources. Furthermore, after applying primary control on voltage and frequency of the microgrid system, there can still be small deviation from nominal value in steady state.Therefore, secondary control is employed in order to restore the DG voltages and frequencies to their nominal value. However, the conventional secondary controls for microgrids assume a centralized structure that requires a complex communication network and reduce the reliability of the system. Hence, proposing new structure for microgrid control along with advanced secondary control algorithm is very important. Additionally, microgrids equipped with cyber components and communication links can also be vulnerable to cyber attacks on critical signals transmitting between the agents. This fact has introduced the essence of investigating novel control approaches and defense strategies in order to increase the resiliency of microrgrid power systems.
\end{abstract}

In order to achieve solutions for the aforementioned challenges, this dissertation starts with modeling of two different and important parts of the distribution power systems, i.e. distribution line and PV systems. Firstly, it studies different approximation methods and develops a new approach for simplification of Carson's equations to model distribution lines for unbalanced power flow and short circuit analysis. The results of applying the proposed method on a three-phase unbalanced distribution system are compared with different existing methods as well as actual impedance values obtained from numerical integration method. Then steady state modeling and optimal placing of multiple PV system are investigated in order to reduce the total loss in the system. The results show the effectiveness of the proposed method in minimizing the total loss in a distribution power system.

The dissertation starts the discussion about microgrid modeling and control by implementing a novel frequency control approach in a microgrid. This study has been carried out step by step by modeling different part of the power system and proposing different algorithms. Firstly, the application of Renewable Energy Sources (RES) 
accompanied with Energy Storage Systems (ESS) in a hybrid system is studied in the presence of Distributed Generation (DG) resources in Load Frequency Control (LFC) problem of microgrid power system with significant penetration of wind speed disturbances. The next step is to investigate the effect of PHEVs in modelling and controlling the microgid. Therefore, system with different penetrations of PHEVs and different stochastic behaviors of PHEVs is modeled. Different kinds of control approaches, including PI control as conventional method and proposed optimal LQR and dynamic programming methods, have been utilized and the results have been compared with each other. Then, Multi Agent System (MAS) is utilized as a control solution which contributes the cyber aspects of microgrid system. The modeled microgrid along with dynamic models of different components is implemented in a centralized multi-agent based structure. The robustness of the proposed controller has been tested against different frequency changes including cyber attack implications with different timing and severity. New attack detection through learning method is also proposed and tested. The results show improvement in frequency response of the microgrid system using the proposed control method and defense strategy against cyber attacks.

Finally, a new multi-agent based control method along with an advanced secondary voltage and frequency control using Particle Swarm Optimization (PSO) and Adaptive Dynamic Programming (ADP) is proposed and tested in the modeled microgrid considering nonlinear heterogeneous dynamic models of DGs. The results are shown and compared with conventional control approaches and different multi-agent structures. It is observed that the results are improved by using the new multi-agent structure and secondary control method.

In summary, contributions of this dissertation center in three main topics. Firstly, new accurate methods for modeling the distribution line impedance and PV system is developed. Then advanced control and defense strategy method for frequency regulation against cyber intrusions and load changes in a microgrid is proposed. Finally, a new hierarchical multi-agent based control algorithm is designed for secondary voltage and frequency control of the microgrid. 


\section{ACKNOWLEDGMENT}

I would like to express my sincere gratitude to my advisor Prof. Jignesh Solanki for the continuous support of my Ph.D study and related research, for his patience, motivation, and immense knowledge. His guidance helped me in all the time of research and writing of this thesis. I could not have imagined having a better advisor and mentor for my Ph.D study.

Besides my advisor, I would like to thank the rest of my thesis committee: Prof. Sarika Khushalani Solanki, Prof. Ali Feliachi, and Prof. Afzel Noore, Prof. Rong luo, and Prof. Hong-Jian Lai for their insightful comments and encouragement, but also for the questions which incented me to widen my research from various perspectives.

I thank my fellow labmates in for the stimulating discussions, for the sleepless nights we were working together before deadlines, and for all the fun we have had in the last four years. Also I thank my friends in WVU.

Last but not the least, I would like to thank my family: my parents and to my brothers and sister for supporting me spiritually throughout writing this dissertation and my wife who has always supported me through this entire journey. 
To dry Eove ofarideh.. 


\section{TABLE OF CONTENTS}

\section{Chapter 1:}

Improving the Accuracy of Impedance Calculation and PV System Modeling for Distribution Power System .................................................................................... 1

Part 1. Improving Distribution Line Calculation ..................................................................... 2

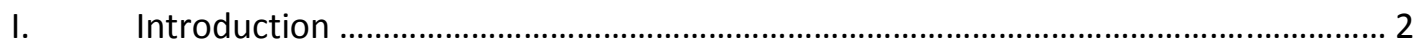

II. Problem Formulation and System Modeling …........................................................ 3

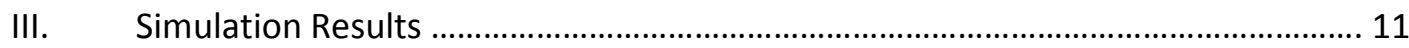

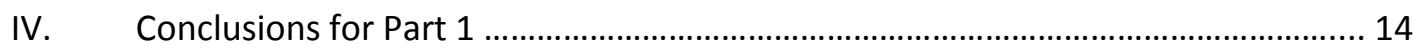

Part 2. Analyzing Multi-Microgrid with Stochastic Uncertainties including Optimal

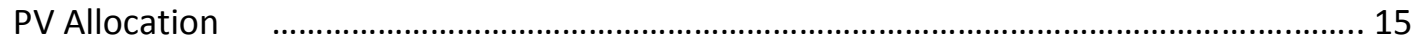

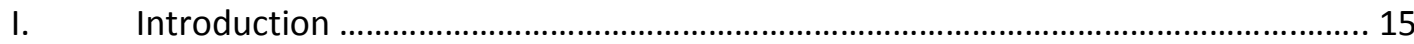

II. Modeling and Problem Formulation ……............................................................ 15

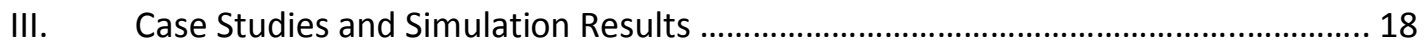

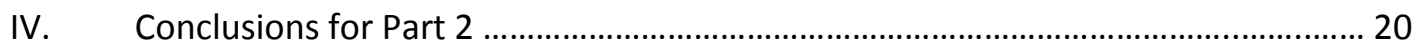

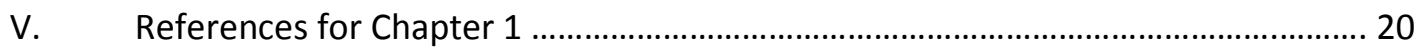

\section{Chapter 2:}

Novel Frequency Control and Defense Strategy against Cyber Intrusions in a Centralized Multiagent based Microgrid ............................................................ 22

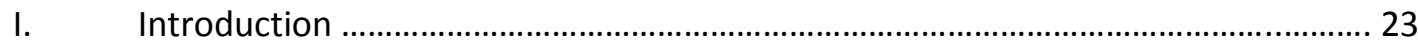

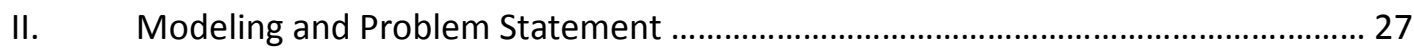

1. LFC for hybrid power generation/energy storage Microgrid system ..................... 27

2. Dynamic Model, Control and Stability Analysis of Microturbine in a Microgrid .... 28 


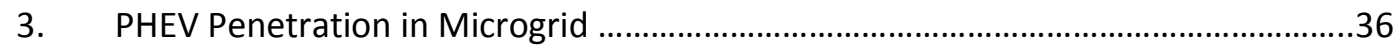

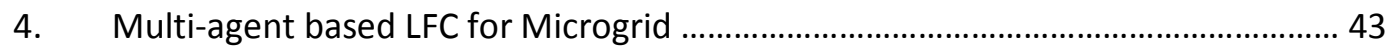

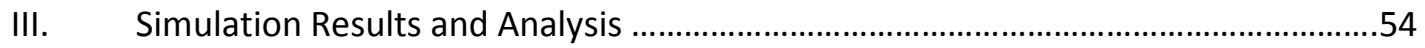

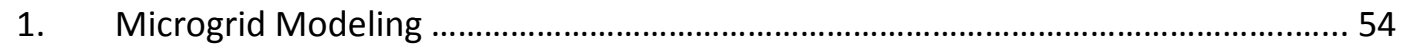

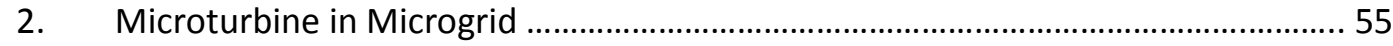

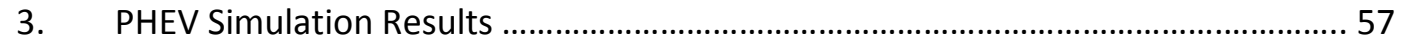

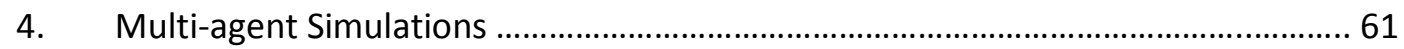

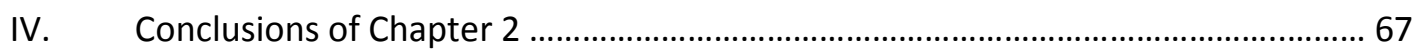

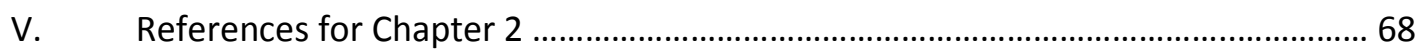

\section{Chapter 3:}

Hierarchical Multi-Agent based Voltage and Frequency Control for a Microgrid

Power System ................................................................... 72

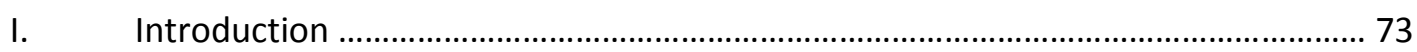

II. Small Signal Modeling and Stability Analysis of the Inverter-based Microgrid ...... 74

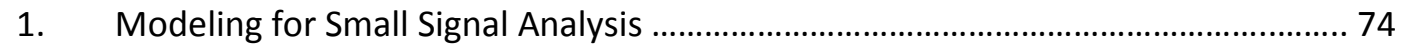

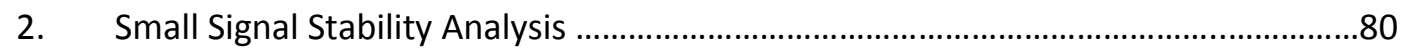

III. Modeling of Multi-Agent based Control of Microgrid .............................................. 84

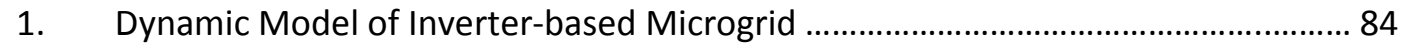

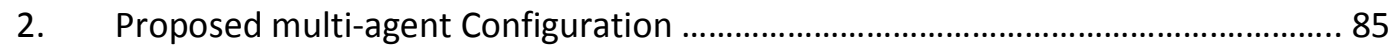

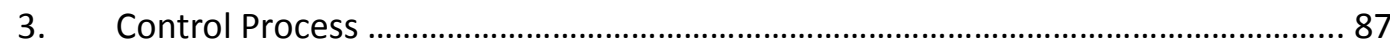

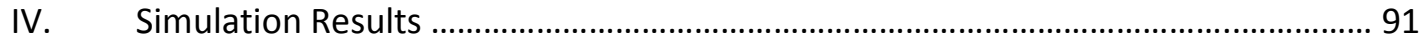

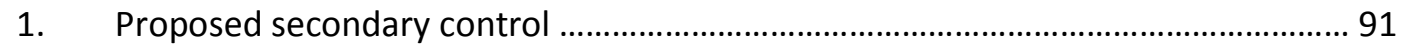

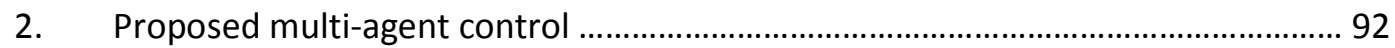

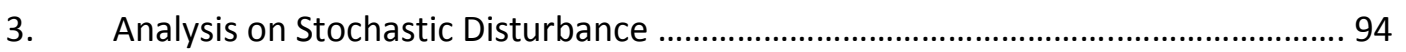

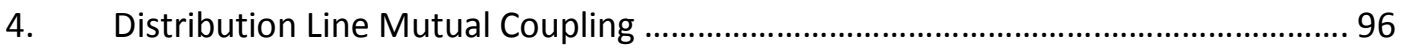


5. Evaluating the results with existing published method ........................................ 97

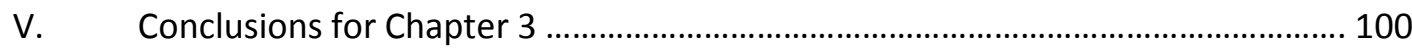

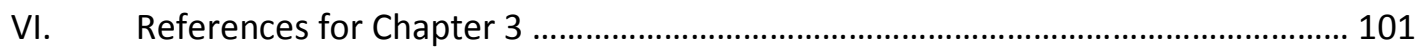

\section{Chapter 4:}

Challenges, Conclusions and Future Work ............................................ 104 


\section{Chapter 1}

\section{Improving the Accuracy of Impedance Calculation and PV System Modeling for Distribution Power System}


In the first part of this chapter, the modeling of the distribution line through approximating the nonlinear infinite integral form of Carson equation is studied and the proposed method is compared with the results of other approximation method as well as numerical integration. Next part of this chapter discusses the modeling of PV in a distribution power system and presents the effects of Photovoltaic (PV) location on losses of the distribution system. The proposed optimal location of PV is determined by using Particle Swarm Optimization (PSO) implemented in MATLAB-OpenDSS environment. IEEE 34-node test feeder is employed to verify the feasibility and effectiveness of the developed method.

\section{Part 1. Improving Distribution Line Calculation}

\section{I- INTRODUCTION}

Calculation of the line parameters, especially series impedance is very critical for analyzing a power system. The impedances are used for modeling underground/ overhead lines and cables of a distribution system and whose accuracy defines the accuracy of unbalanced distribution power flow solutions. The accuracy of fault analysis in time-domain calculations is also affected by the assumptions in the evaluation of the distribution line impedance [1]. The complexities of the exact representation of the distribution line parameters have led investigators to propose approximate formulae, and several of these are available in technical literature.

Initially, impedance calculations were based exclusively on the geometry. Later, skin and proximity effect were taken into account [2-4]. In 1926 Carson [5] proposed a method of calculating the influence of imperfect earth on transmission lines and showed that modeling ground returns affects the accuracy of impedances and hence the voltage rise and drop [6]. Several authors [7-10] have developed expressions for the ground return parameters, but most widely used are Carson's equations for overhead lines and Pollaczeck's [8] equations for underground cables. Carson's [7] equations were used for decades, before new approaches for these calculations were made available to the scientific community [11-14]. However, Carson's equations contain infinite integrals with complex arguments which make them difficult to be computationally analyzed. Hence, converging infinite series was used widely and most handbook formulas were derived from these series. The integral terms of Carson's equations can be evaluated by infinite series [15] that contains exponential terms which can be further approximated at low and high frequencies [16-19]. These approximations and impedance calculations are investigated and compared using unbalanced power flow results [42].

The organization of this chapter is as follows: Section II discusses different impedance calculations method used in simulation section. A new impedance calculation method is proposed in this section. In Section III, the application of approximation methods for calculating the impedance of the system, including longest distribution line and a load, along with power flow analysis, is investigated by simulations in MATLAB ${ }^{\mathrm{TM}}$ software. Application of different impedance calculation methods is investigated in impedance, power flow and short circuit results of 123 node distribution power system using OpenDSS software. The effect of ground resistivity and frequency in line impedance results are investigated in the next part of simulations. Finally, the chapter is concluded in Section IV. 


\section{KESHTKAR: MODELING AND CONTROLLING A HYBRID MULTI-AGENT BASED MICROGRID IN PRESENCE OF DIFFERENT PHYSICAL AND CYBER COMPONENTS}

\section{II- Problem Formulation ANd System Modeling}

The impedance calculation of distribution lines/cables affects the accuracy of steady state, transient and fault analysis of distributions systems. The impedance calculation has thus been studied for several decades; in particular, some methods are analyzed in this chapter, including a proposed method. Section A presents the integral terms in impedance calculation that needs to be approximated and section $\mathrm{B}$ presents the approximation of these integral by different methods. In section $\mathrm{C}$, there is an evaluation of different methods based on math formulation before simulation results in the next section.

\section{A. Integral mode of impedance calculation}

Impedance calculations include the computation of self and mutual impedance of a conductor. Equations (1.1) and (1.2) have been widely used in literature for calculating self and mutual impedances of ideal conductors.

$$
\begin{aligned}
& Z_{s}=j \omega \frac{\mu_{0}}{2 \pi} \ln \frac{2 h_{s}}{r}+j \omega \frac{\mu}{\pi} J_{s}(\Omega) \\
& Z_{m}=j \omega \frac{\mu_{0}}{2 \pi} \ln \frac{\sqrt{\left(h_{k}+h_{m}\right)^{2}+d^{2}}}{\sqrt{\left(h_{k}-h_{m}\right)^{2}+d^{2}}}+j \omega \frac{\mu}{\pi} J_{m}(\Omega)
\end{aligned}
$$

where

$$
\begin{aligned}
& J_{s}=P_{s}+j Q_{s}=\int_{0}^{\infty} \frac{e^{-2 h_{p} \lambda}}{\lambda+\sqrt{\lambda^{2}+j \omega \mu / \rho}} \cos (\lambda d) d \lambda \\
& J_{m}=P_{m}+j Q_{m}=\int_{0}^{\infty} \frac{e^{-\left(h_{k}+h_{m}\right) \lambda}}{\lambda+\sqrt{\lambda^{2}+j \omega \mu / \rho}} \cos (\lambda d) d \lambda
\end{aligned}
$$

where,

$\omega$ complex frequency, rad/s

$\mu_{r}$ relative magnetic permeability of the ground

$\rho$ resistivity of the ground,ohm-meter

$r \quad$ radius of the conductor, $m$ (self)

$h_{p}, h_{k}, h_{m}$ height of the conductors $p, k, m$

$d$ horizontal seperation of the conductor, $m$.

A new dimensionless complex integration variable $w$ is introduced, which is $w=\lambda \cdot p$. Substituting for $\lambda$ from equation (1.5), $\mathrm{p}$ from equation (1.6), and defining $\mathrm{h} / \mathrm{p}=\mathrm{q}$ in equation (1.3), we get equation (1.7):

$$
\begin{aligned}
& \lambda=\frac{w}{p} \\
& p=\frac{1}{\sqrt{j \omega \mu \sigma}} \\
& J_{s}(q)=\int_{0}^{\infty} \frac{2 w e^{-2 q w}}{w+\sqrt{w^{2}+1}} d w
\end{aligned}
$$

Using Substituting for $\mathrm{q}$ and $\beta$ from equations (1.8) and (1.9) in equation (1.4), we get equation (1.10)

$$
\begin{aligned}
& q=\frac{h_{k}+h_{l}}{2 p} \\
& \beta=\frac{d_{k l}}{h_{k}+h_{l}} \\
& J_{m}(q)=\frac{1}{2} \int_{0}^{\infty} \frac{e^{-2(1+j \beta) q w}+e^{-2(1-j \beta) q w}}{w+\sqrt{w^{2}+1}} d w
\end{aligned}
$$

Equations (1.3) and (1.4), or (1.7) and (1.10) are the infinite integral terms in self and mutual impedances equations. Different methods discussed in parts B1-B9 are used in order to approximate these infinite integral terms. 


\section{B.1 Full Carson}

Solution of equations (1.3) and (1.4) requires numerical methods that are computationally intensive and hence simplifications are needed. Carson's closed-form approximation to these integrals leads to equations (1.11)-(1.16) [20]:

$$
\begin{aligned}
X_{i} & =2 \omega G \cdot \ln \frac{R D_{i}}{G M R_{i}} \Omega / \text { mile } \\
P_{i j} & =\frac{\pi}{8}-\frac{1}{3 \sqrt{2}} k_{i j} \cos \left(\theta_{i j}\right) \cdot\left(0.6728+\ln \frac{2}{k_{i j}}\right) \\
Q_{i j} & =-0.0386+\frac{1}{2} \cdot \ln \frac{2}{k_{i j}}+\frac{1}{3 \sqrt{2}} k_{i j} \cos \left(\theta_{i j}\right) \\
k_{i j} & =8.565 \times 10^{-4} \times S_{i j} \times \sqrt{\frac{f}{\rho}} \\
\hat{Z}_{i i} & =r_{i}+4 \omega P_{i i} G+j\left(X_{i}+2 \omega G \cdot \ln \frac{S_{i i}}{R D_{i}}+4 \omega Q_{i i} G\right) \Omega / \text { mile } \\
\hat{Z}_{i j} & =4 \omega P_{i j} G+j\left(2 \omega G \cdot \ln \frac{S_{i j}}{R D_{i j}}+4 \omega Q_{i j} G\right) \Omega / \text { mile }
\end{aligned}
$$

where,

$Z_{i i}$ : self-impedance of conductor i in $\Omega /$ mile

$Z_{i j}$ : mutual-impedance between conductors $i$ and $j$ in $\Omega /$ mile

$R_{i}$ : Resistance of conductor i in $\Omega /$ mile

$\omega=2 \pi f:$ is system angular frequency in radians per second

G: $0.1609344 .10^{-3} \Omega /$ mile

$R D$ : radius of conductor $i$ in feet

$G M R_{i}:$ Geometric Mean Radius of conductor i in feet

$f:$ system frequency in hertz

$\rho:$ resistivity of earth in ohm - meter

$D_{i j}$ : distance between conductors $i$ and $j$ in feet

$S_{i j}:$ distance between conductors $i$ and image $j$ in feet

$\theta_{i j}$ : angle between a pair of lines drawn from conductor ito its

own image and to the image of conductor $j$

\section{B.2 Modified Carson}

$\mathrm{P}_{\mathrm{ij}}$ and $\mathrm{Q}_{\mathrm{ij}}$, shown in equations (1.12) and (1.13) were further approximated by Carson considering only first term in $\mathrm{P}_{\mathrm{ij}}$ and first two terms in $\mathrm{Q}_{\mathrm{ij}}$ as shown by equations (1.17) and (1.18).

$$
\begin{aligned}
& P_{i i}=P_{i j}=\pi / 8 \\
& Q_{i i}=Q_{i j}=-0.03860+\frac{1}{2} \ln \frac{2}{k_{i j}}
\end{aligned}
$$

In the above approximations, frequency is $60 \mathrm{Hertz}$ and Earth resistivity is $100 \mathrm{Ohm}$-meter.

Substituting $\mathrm{P}_{\mathrm{ij}}$ and $\mathrm{Q}_{\mathrm{ij}}$ from equations (1.17) and (1.18) in (1.15) and (1.16), the equations for self and mutual impedances are obtained as given by equations (1.19) and (1.20):

$$
\begin{aligned}
& \hat{Z}_{i i}=r_{i}+0.09530+j 0.12134\left(\ln \frac{1}{G M R_{i}}+7.93402\right) \Omega / \text { mile } \\
& \hat{Z}_{i j}=0.09530+j 0.12134\left(\ln \frac{1}{D_{i j}}+7.93402\right) \Omega / \text { mile }
\end{aligned}
$$

\section{B.3 Deri}

For modeling line impedances Deri introduces the concept of an ideal (superconducting) current return plane placed below the ground surface at complex distance $\rho$ equal to the complex penetration depth for plane waves. This "complex" plane appears as a mirroring surface, so that conductor images can be used to derive very simple formulae for self and mutual impedances. The 
KESHTKAR: MODELING AND CONTROLLING A HYBRID MULTI-AGENT BASED MICROGRID IN PRESENCE OF DIFFERENT PHYSICAL AND CYBER COMPONENTS

ideal current return plane at complex depth can also be used for multi-layer earth return path. Furthermore, Deri used equation (1.21) to approximate the integral expression in equation (1.7)

$$
\frac{2 w}{w+\sqrt{w^{2}+1}} \cong 1-e^{-2 w}
$$

Using ideal current return plane as the reference for the conductor distances and considering approximation in equation (1.21) the impedance equations (1.1), (1.2), can be written as shown in equations (1.22) and (1.23):

Self impedance:

$$
Z_{s} \cong \pi^{2} f 10^{-7}+j \omega \frac{\mu_{0}}{2 \pi} \ln \frac{711.8}{r \sqrt{f \sigma}}
$$

Mutual impedance:

$$
Z_{m}=j \omega \frac{\mu_{0}}{2 \pi} \ln \frac{\sqrt{\left(h_{k}+h_{m}\right)^{2}+d^{2}}}{\sqrt{\left(h_{k}-h_{m}\right)^{2}+d^{2}}}+j \omega \frac{\mu}{\pi} J_{m}
$$

$\mathrm{Z}_{\mathrm{m}}$ in equation (1.2) is similar to equation (1.23), however $\mathrm{J}_{\mathrm{m}}$ is different from equation (1.4) and it is given by equation (1.24)

$$
J_{m}(q) \cong \frac{j}{2} \ln \frac{\sqrt{(1+1 / q)^{2}+\beta^{2}}}{\sqrt{1+\beta^{2}}}, q=\frac{h_{k}-h_{l}}{2 \rho}, \quad \beta=\frac{d_{k l}}{h_{k}-h_{l}}
$$

\section{B.4 Alvarado}

Alvarado proved that Deri's equations particularly for large $\mathrm{d} / \mathrm{h}$, in equation (1.2) where $\mathrm{d}$ is horizontal distance between phases and $\mathrm{h}$ is the height of the conductor, does not provide accurate solutions of power flow analysis at certain frequencies. At higher frequencies $\mathrm{d} / \mathrm{h}$ is large and results in errors in impedances to be as high as 15 percent [21]. Unlike Deri who used equation (1.21) to approximate equation (1.7), Alvarado used equation (1.25) to approximate equation (1.7).

$$
\frac{2 w}{w+\sqrt{w^{2}+1}} \cong 1-e^{-2 w}-\frac{1}{3} w^{3} e^{-2 w}
$$

Approximation terms in equation (1.21) by Deri and (1.25) by Alvarado are compared to actual terms in equation (1.7) and are shown in Fig. 1.1. It can be seen from Fig. 1.1 that Alvarado's proposed approximation matches closely to the accurate value than Deri's method.

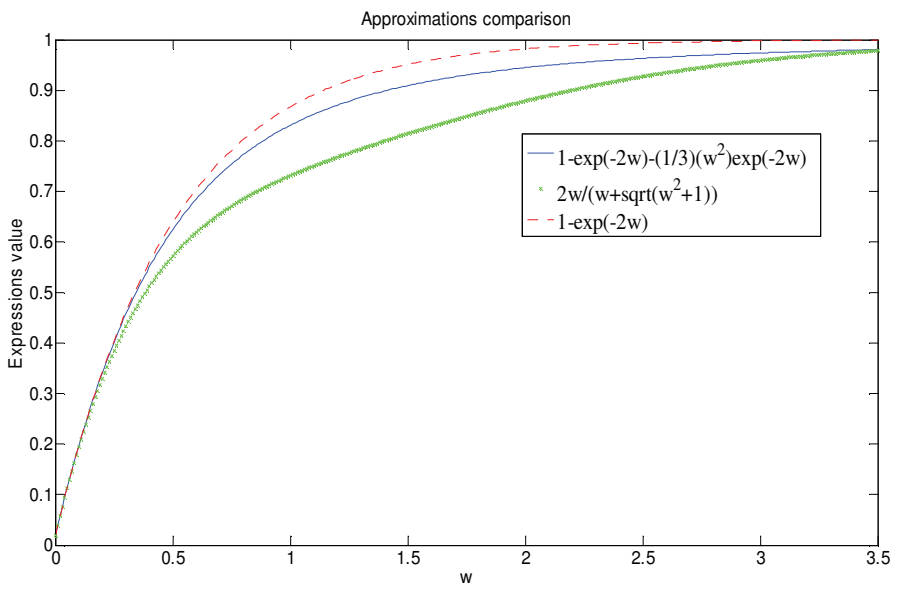

Fig. 1.1. Comparison of approximation used in Deri's and Alvarado's impedance calculation method

Substituting equation (1.25) in equations (1.7) and (1.10), we get equations (1.26) and (1.27): 


$$
\begin{gathered}
J_{s} \cong \frac{1}{2} \ln \left(\frac{p}{h_{s}}+1\right)-\frac{1}{24} \frac{1}{\left(1+\frac{h_{s}}{p}\right)^{3}} \\
J_{m}=\frac{1}{4} \ln \left(\frac{\left(1+\frac{p}{h}\right)^{2}+\beta^{2}}{1+\beta^{2}}\right)-\frac{1}{48}\left(\frac{1}{\left[\frac{h}{p}(1+j \beta)+1\right]^{3}}+\frac{1}{\left[\frac{h}{p}(1-j \beta)+1\right]^{3}}\right) \\
\text { where, } h=\frac{h_{k}+h_{m}}{2} \text { and } p=\frac{1}{\sqrt{j}} \sqrt{\frac{\rho}{\omega \mu}}
\end{gathered}
$$

Equations (1.26) and (1.27) can be substituted in equation (1.29) and (1.30), which are similar to equations (1.1) and (1.2), to get $\mathrm{Z}_{\mathrm{s}}$ and $\mathrm{Z}_{\mathrm{m}}$ :

$$
\begin{aligned}
& Z_{s}=j \omega \frac{\mu_{0}}{2 \pi} \ln \frac{2 h_{s}}{r}+j \omega \frac{\mu}{\pi} J_{s}(\Omega) \\
& Z_{m}=j \omega \frac{\mu_{0}}{2 \pi} \ln \frac{\sqrt{\left(h_{k}+h_{m}\right)^{2}+d^{2}}}{\sqrt{\left(h_{k}-h_{m}\right)^{2}+d^{2}}}+j \omega \frac{\mu}{\pi} J_{m}(\Omega)
\end{aligned}
$$

\section{B.5 Noda}

Noda's method is a simple closed-form approximate of the ground-return impedance of horizontal parallel wires above a lossy ground plane [22]. It is derived by a single logarithmic approximation of the equations with a semi-infinite interval in Carson's ground-return impedance formula. Unlike Deri and Alvarado [21] who approximate equations (1.7) and (1.10) for calculating impedance integral formulas, Noda approximates integral expression in equations (1.3) and (1.4), and substitutes them in equations (1.1) and (1.2) to get equations (1.31) and (1.32)

$$
\begin{aligned}
& Z_{s}=\frac{j \omega \mu_{0}}{2 \pi}\left[\ln \frac{2 h_{i}}{r_{i}}+I\right] \\
& Z_{m}=\frac{j \omega \mu_{0}}{2 \pi}\left[\ln \frac{\sqrt{\left(h_{i}+h_{j}\right)^{2}+x_{i j}^{2}}}{\sqrt{\left(h_{i}-h_{j}\right)^{2}+x_{i j}^{2}}}+I\right] \\
& \text { where, } I=\int_{0}^{\infty} \frac{2 e^{-\left(h_{i}+h_{j}\right) \lambda}}{\lambda+\sqrt{\lambda^{2}+j \Omega}} \cos \left(x_{i j} \lambda\right) d \lambda, \Omega=\omega \mu_{0} \sigma
\end{aligned}
$$

In these equations, $\omega$ is angular frequency, $\mathrm{r}_{\mathrm{i}}$ and $\mathrm{h}_{\mathrm{i}}$ are the radius and the height of the $\mathrm{i}^{\mathrm{ith}}$ conductor and $\mathrm{x}_{\mathrm{ij}}$ is the horizontal separation between the $\mathrm{i}^{\text {th }}$ and the $\mathrm{j}^{\text {th }}$ conductor.

Noda further uses the single logarithmic approximation for equations (1.31) and (1.32) to get equations (1.34) and (1.35).

$$
\begin{aligned}
Z_{s}= & j \omega \frac{\mu_{0}}{2 \pi}\left[A \ln \frac{\sqrt{\left(2 h_{i}+2 \alpha p\right)^{2}}}{r_{i}}+(1-A) \ln \frac{\sqrt{\left(2 h_{i}+2 \beta p\right)^{2}}}{r_{i}}\right] \\
Z_{m}= & j \omega \frac{\mu_{0}}{2 \pi}\left[A \ln \frac{\sqrt{\left(h_{i}+h_{j}+2 \alpha p\right)^{2}+x_{i j}{ }^{2}}}{\sqrt{\left(h_{i}-h_{j}\right)^{2}+x_{i j}{ }^{2}}}\right. \\
& \left.+(1-A) \ln \frac{\sqrt{\left(h_{i}+h_{j}+2 \beta p\right)^{2}+x_{i j}{ }^{2}}}{\sqrt{\left(h_{i}-h_{j}\right)^{2}+x_{i j}{ }^{2}}}\right]
\end{aligned}
$$


KESHTKAR: MODELING AND CONTROLLING A HYBRID MULTI-AGENT BASED MICROGRID IN PRESENCE OF DIFFERENT PHYSICAL AND CYBER COMPONENTS

$$
\begin{aligned}
& \text { where, } \theta=\tan ^{-1} \frac{x}{H} \quad \text { deg } \\
& A= \begin{cases}0.07360 & \left(\theta \leq 50.45^{\circ}\right) \\
0.002474 \theta-0.05127 & \left(\theta \succ 50.45^{\circ}\right)\end{cases} \\
& \alpha= \begin{cases}0.1500 & \left(\theta \leq 50.45^{\circ}\right) \\
0.004726 \theta-0.08852 & \left(\theta \succ 50.45^{\circ}\right)\end{cases} \\
& \beta=\frac{1-A \alpha}{1-A} \\
& p=\frac{1}{\sqrt{j \omega \mu_{0} \sigma}}
\end{aligned}
$$

\section{B.6 Pizarro and Eriksson:}

Unlike Noda that uses single logarithmic approximation to get equations (1.34) and (1.35), Pizzaro [23] and Eriksson proposed double logarithmic approximation which results in equations (1.37) and (1.38) [25]:

$$
\begin{aligned}
Z_{s}= & j \omega \frac{\mu_{0}}{2 \pi}\left[A \ln \frac{\sqrt{\left(2 h_{i}+2 \alpha p\right)^{2}}}{r_{i}}+B \ln \frac{\sqrt{\left(2 h_{i}+2 \beta p\right)^{2}}}{r_{i}}\right] \\
Z_{m}= & j \omega \frac{\mu_{0}}{2 \pi}\left[A \ln \frac{\sqrt{\left(h_{i}+h_{j}+2 \alpha p\right)^{2}+x_{i j}^{2}}}{\sqrt{\left(h_{i}-h_{j}\right)^{2}+x_{i j}^{2}}}\right. \\
& \left.+B \ln \frac{\sqrt{\left(h_{i}+h_{j}+2 \beta p\right)^{2}+x_{i j}^{2}}}{\sqrt{\left(h_{i}-h_{j}\right)^{2}+x_{i j}^{2}}}\right]
\end{aligned}
$$

where the constants $A=0.1159, B=0.8841, \alpha=0.2258$, and $\beta=1.1015$ are determined by a least-squares process using the Prony algorithm [24].

\section{B.7 Dubanton}

A new heuristic justification of the concept of a ground-current return plane placed at a complex depth has been suggested by Dubanton and proved by Gary. The method of the "complex ground return plane" is extended for the modeling of multi-layer ground return. Using this plane as the reference of conductor distances, unlike Deri who approximated equation (1.7) and (1.10), Dubanton approximated equations (1.3) and (1.4) and substituted in equations (1.1) and (1.2) to get equations (1.39) and (1.40) which were valid for the whole range of frequencies.

$$
\begin{aligned}
& Z_{s}=j \omega \frac{\mu_{0}}{2 \pi} \ln \frac{2(h+p)}{r} \\
& Z_{m}=j \omega \frac{\mu_{0}}{2 \pi} \ln \frac{\sqrt{\left(h_{k}+h_{l}+2 p\right)^{2}+d_{k l}^{2}}}{\sqrt{\left(h_{k}-h_{l}\right)^{2}+d_{k l}^{2}}}
\end{aligned}
$$

\section{B.8 Expanding of Full Carson method}

Full Carson method, inherently, is an infinite series formula. The complete formula for this method is shown in equation (1.41) and (1.42).

$$
\begin{aligned}
& P=\frac{\pi}{8}\left(1-s_{4}\right)+\frac{1}{2}\left(\log \frac{2}{\gamma}\right) s_{2}+\frac{1}{2} \theta \cdot s_{2}^{\prime}-\frac{1}{\sqrt{2}} \sigma_{1}+\frac{1}{2} \sigma_{2}+\frac{1}{\sqrt{2}} \sigma_{3} . \\
& Q=\frac{1}{4}+\frac{1}{2}\left(\log \frac{2}{\gamma}\right)\left(1-s_{4}\right)-\frac{1}{2} \theta \cdot s_{4}^{\prime}+\frac{1}{\sqrt{2}} \sigma_{1}-\frac{\pi}{8} s_{2}+\frac{1}{\sqrt{2}} \sigma_{3}-\frac{1}{2} \sigma_{4} .
\end{aligned}
$$




$$
\begin{aligned}
& \text { where, } \\
& \gamma=1.7811, \log \frac{2}{\gamma}=0.11593, \log \gamma=0.57722 \\
& s_{2}=\frac{1}{1 ! 2 !}\left(\frac{r}{2}\right)^{2} \cos 2 \theta-\frac{1}{3 ! 4 !}\left(\frac{r}{2}\right)^{6} \cos 6 \theta+\ldots \\
& s_{2}^{\prime}=\frac{1}{1 ! 2 !}\left(\frac{r}{2}\right)^{2} \sin 2 \theta-\frac{1}{3 ! 4 !}\left(\frac{r}{2}\right)^{6} \sin 6 \theta+\ldots \\
& s_{4}=\frac{1}{2 ! 3 !}\left(\frac{r}{2}\right)^{4} \cos 4 \theta-\frac{1}{4 ! 5 !}\left(\frac{r}{2}\right)^{8} \cos 8 \theta+\ldots \\
& s_{4}^{\prime}=\frac{1}{2 ! 3 !}\left(\frac{r}{2}\right)^{4} \sin 4 \theta-\frac{1}{4 ! 5 !}\left(\frac{r}{2}\right)^{8} \sin 8 \theta+\ldots \\
& \sigma_{1}=\frac{r \cos \theta}{3}-\frac{r^{5} \cos 5 \theta}{3^{2} \cdot 5^{2} \cdot 7}+\frac{r^{9} \cos 9 \theta}{3^{2} \cdot 5^{2} \cdot 7^{2} \cdot 9^{2} \cdot 11}-\ldots \\
& \sigma_{2}=\left(1+\frac{1}{2}-\frac{1}{4}\right) \frac{1}{1 ! 2 !}\left(\frac{r}{2}\right)^{2} \cos 2 \theta-\ldots=\frac{5}{4} s_{2} \text { approximately. }
\end{aligned}
$$

Using three terms in Full Carson method has been shown in (1.11) - (1.16). Using six terms in this calculation we will have equations (1.44) and (1.45) instead of (1.12) and (1.13)

$$
\begin{aligned}
P_{i j}= & \frac{\pi}{8}-\frac{1}{3 \sqrt{2}} k_{i j} \cos \left(\theta_{i j}\right)+\frac{k_{i j}{ }^{2} \cos \left(2 \theta_{i j}\right)}{16} \cdot\left(0.6728+\ln \frac{2}{k_{i j}}\right) \\
& +\frac{k_{i j}{ }^{2}}{16} \cdot \theta_{i j} \sin \left(\theta_{i j}\right)+\frac{k_{i j}{ }^{3}}{\sqrt{2} \cdot 45} \cdot \cos \left(3 \theta_{i j}\right)-\frac{\pi \cdot k_{i j}{ }^{4}}{1536} \cdot \cos \left(4 \theta_{i j}\right) \\
Q_{i j}= & -0.0386+\frac{1}{2} \cdot \ln \frac{2}{k_{i j}}+\frac{1}{3 \sqrt{2}} k_{i j} \theta_{i j}-\frac{\pi \cdot k_{i j}{ }^{2}}{64} \cdot \cos \left(2 \theta_{i j}\right) \\
+ & \frac{k_{i j}{ }^{3}}{\sqrt{2} \cdot 45} \cdot \cos \left(3 \theta_{i j}\right)-\frac{k_{i j}{ }^{4}}{384} \cdot \theta_{i j} \cdot \sin \left(4 \theta_{i j}\right)-\frac{k_{i j}{ }^{4} \cos \left(4 \theta_{i j}\right)}{384} \cdot\left(1.0895+\ln \frac{2}{k_{i j}}\right)
\end{aligned}
$$

Keeping the impedance calculated by Full Carson with six terms as reference the error in impedance calculated with three terms of Full Carson is shown in Table 1.I.

It can be seen that if the accuracy of the results are important, using more accurate version of Full Carson method seems necessary.

Table 1.I. Percentage deviation of three terms Full Carson with six terms as reference
\begin{tabular}{|l|l|l|l|}
\hline Zi1 & Zi2 & Zi3 & Zi4 \\
\hline $0.1506-0.3919 \mathrm{i}$ & $0.1728-0.3966 \mathrm{i}$ & $0.1861-0.3983 \mathrm{i}$ & $0.0512-0.3003 \mathrm{i}$ \\
\hline $0.1512-0.3917 \mathrm{i}$ & $0.1768-0.3990 \mathrm{i}$ & $0.1809-0.3933 \mathrm{i}$ & $0.0499-0.2984 \mathrm{i}$ \\
\hline $0.1483-0.3888 \mathrm{i}$ & $0.1642-0.3888 \mathrm{i}$ & $0.1943-0.4040 \mathrm{i}$ & $0.0520-0.3002 \mathrm{i}$ \\
\hline $0.1320-0.3403 \mathrm{i}$ & $0.1488-0.3426 \mathrm{i}$ & $0.1657-0.3482 \mathrm{i}$ & $0.0386-0.2556 \mathrm{i}$ \\
\hline
\end{tabular}

\section{B.9 Proposed method for solving integral form of Carson method}

Using some calculations and variable changes integral equations (1.1)-(1.4) can be expressed by equation (1.46).

$$
\begin{aligned}
& f(\varepsilon)=-2 j \int_{0}^{\infty} \sqrt{\left(1-\frac{t^{2}}{4}\right)} e^{-j t \varepsilon} d t-\int_{0}^{\infty} \sqrt{\left(1+\frac{t^{2}}{4}\right)-\frac{t}{2}} e^{-t \varepsilon} d t \\
& \text { where, } \\
& \varepsilon_{1,2}=\left(\frac{-h_{k}+h_{l}}{2 \delta} \pm j \frac{H_{k l}}{2 \delta}\right)(1+j)
\end{aligned}
$$

After using infinite series to solve this new integral form, equations (1.48)-(1.50) are obtained. 
KESHTKAR: MODELING AND CONTROLLING A HYBRID MULTI-AGENT BASED MICROGRID IN PRESENCE OF DIFFERENT PHYSICAL AND CYBER COMPONENTS

$$
\begin{aligned}
& Z_{m}=\frac{1}{2} j \omega\left(g\left(\varepsilon_{1}\right)+g\left(\varepsilon_{2}\right)\right) \\
& g(\varepsilon)=X+\sqrt{\pi j} \frac{e^{-2 j \varepsilon}}{\varepsilon \sqrt{\varepsilon}}\left(1-j \frac{3}{16 \varepsilon}+\frac{5}{512 \varepsilon^{2}}+\ldots\right) \\
& X=-2\left[\frac{1}{(2 \varepsilon)^{2}}+\frac{1}{2 \varepsilon}+\frac{1}{(2 \varepsilon)^{3}}-\frac{3}{(2 \varepsilon)^{5}}+\frac{3^{2} \cdot 5}{(2 \varepsilon)^{7}}-\frac{3^{2} \cdot 5^{2} \cdot 7}{(2 \varepsilon)^{9}}+\ldots\right]
\end{aligned}
$$

These equations can be used in computational calculation of line impedances. The accuracy of this proposed method is investigated in the next section.

\section{Evaluation of the methods based on math formulation}

Carson methods and the proposed method have the most accurate results based on using infinite series solutions for integral form of impedance formula. Among other approximation methods, Alvarado and Deri for having more terms in the approximations, Noda for using adjustable parameters for different configurations and Pizzaro for using optimized parameters in calculation have the next accuracies. These claims will be investigated in simulation results section.

In continue of math evaluation of the methods, a comparison between the approximation methods and the exact impedance value of one sample line obtaining from numerical integration has been made in section III.D for different range of frequencies.

In order to make a comparison between the approximation methods and the exact values, a mathematical formulation has been used to calculate the exact value of integral form of line impedance presented in (1.3) and (1.4). This numerical integration approach is discussed in the rest of this section.

\section{Evaluating the approximation methods with numerical integration}

To evaluate the integral in equation (1.3) using numerical method the higher limit of integral is changed to $\lambda_{\max }$ and the integral with the new finite higher bound is shown in equation (1.51). The truncated part of the integral is estimated as shown in equation (1.52). Starting with $\lambda_{\max }=1$, in an iterative approach $\lambda_{\max }$ is increased until the relative-error in (1.53) is less than a given tolerance.

$$
\begin{aligned}
& I^{\prime}=\int_{0}^{\lambda_{\max }} \frac{2 e^{-2 h_{p} \lambda}}{\lambda+\sqrt{\lambda^{2}+j \omega \mu / \rho}} \cos (\lambda d) d \lambda \\
& I_{\text {res }}=\left|\frac{2}{\lambda_{\text {max }}+\sqrt{\lambda_{\text {max }}^{2}+j \omega \mu / \rho}}\right| \int_{\lambda_{\text {max }}}^{\infty} e^{-2 h_{p} \lambda} d \lambda \\
&=\frac{1}{2 h_{p}}\left|\frac{2}{\lambda_{\text {max }}+\sqrt{\lambda_{\text {max }}^{2}+j \omega \mu / \rho}}\right| e^{-2 h_{p} \lambda_{\text {max }}} \\
& \mid I_{\text {res }} / I^{\prime}
\end{aligned}
$$

For evaluating (1.51) an adaptive Gauss/Lobatto quadrature rule is used [26]. The adaptive Gauss/Lobatto quadrature rule requires an absolute tolerance rather than a relative one, and absolute tolerance is obtained by multiplying $\varepsilon$ by $\left|I^{\prime}\right|$ calculated at the previous iteration step. All the calculations here use $\varepsilon=10^{-6}$.

The results for three different IEEE line configurations, obtained by different approximation methods and the exact numerical integration method in $\mathrm{f}=60 \mathrm{~Hz}$, are shown in Table 1.II. Configuration 1 is related to a long distribution line presented in section 
III.A, configuration 2 is one of the IEEE 123 node line configurations used in section III.B, and configuration 3 is taken from IEEE 13 node system.

Table 1.II. Maximum errors in different line impedances for different impedance calculation methods

\begin{tabular}{|c|c|c|c|}
\hline \multirow{2}{*}{ Impedance } & \multicolumn{3}{|c|}{ Max error in line impedances (\%) } \\
\cline { 2 - 4 } & Config.1 & Config.2 & Config.3 \\
\hline $\begin{array}{c}\text { Numerical } \\
\text { Integration }\end{array}$ & $0.0000+0.0000 \mathrm{i}$ & $0.0000+0.0000 \mathrm{i}$ & $0.0000+0.0000 \mathrm{i}$ \\
\hline Proposed Method & $0.0096+0.0045 \mathrm{i}$ & $0.0182+0.0047 \mathrm{i}$ & $0.0083+0.0039 \mathrm{i}$ \\
\hline Deri & $0.6962-0.3391 \mathrm{i}$ & $0.7125-0.3738 \mathrm{i}$ & $0.5347-0.2604 \mathrm{i}$ \\
\hline Alvarado & $0.6193+0.0643 \mathrm{i}$ & $0.6433+0.0612 \mathrm{i}$ & $0.4793+0.04471 \mathrm{i}$ \\
\hline Noda & $0.7296+0.0011 \mathrm{i}$ & $0.7439+0.0012 \mathrm{i}$ & $0.5603+0.0009 \mathrm{i}$ \\
\hline Pizzaro & $0.6621-0.0793 \mathrm{i}$ & $0.6984-0.0765 \mathrm{i}$ & $\begin{array}{c}0.5288- \\
0.05827 \mathrm{i}\end{array}$ \\
\hline Dubanton & $0.6082-0.0741 \mathrm{i}$ & $0.6648-0.0749 \mathrm{i}$ & $\begin{array}{c}0.4602- \\
0.05581 \mathrm{i}\end{array}$ \\
\hline
\end{tabular}

By using numerical integration method as reference the comparison between the maximum errors of different approximation methods has been made in Table 1.II. As it is mathematically proved in this section, proposed method has the most accurate results among impedance approximation methods. In the next section this claim will be investigated by different simulations.

Table 1.III. The results of Carson's full and modified methods, proposed method, and five different approximation methods

\begin{tabular}{|l|}
\hline Z_Full_Carson_6 term: \\
$8.617003+11.5171 \mathrm{i} 0.662456+6.0628 \mathrm{i} 0.662453+5.5563 \mathrm{i}$ \\
$0.662456+6.0628 \mathrm{i} 8.617003+11.5171 \mathrm{i} 0.662448+5.1755 \mathrm{i}$ \\
$0.662453+5.5563 \mathrm{i} 0.662448+5.1755 \mathrm{i} 8.617003+11.5171 \mathrm{i}$ \\
\hline Z_Full_Carson_3 term: \\
8.617002 + 11.5172i $0.662455+6.0629 \mathrm{i} 0.662451+5.5564 \mathrm{i}$ \\
$0.662455+6.0629 \mathrm{i} 8.617002+11.5172 \mathrm{i} 0.662444+5.1756 \mathrm{i}$ \\
$0.662451+5.5564 \mathrm{i} 0.662444+5.1756 \mathrm{i} 8.617002+11.5172 \mathrm{i}$ \\
\hline Z_Modified_Carson: \\
8.631392+11.5019i $0.676846+6.0478 \mathrm{i} 0.676846+5.5412 \mathrm{i}$ \\
$0.676846+6.0478 \mathrm{i} 8.631392+11.5019 \mathrm{i} 0.676846+5.1604 \mathrm{i}$ \\
$0.676846+5.5412 \mathrm{i} 0.676846+5.1604 \mathrm{i} 8.631392+11.5019 \mathrm{i}$ \\
\hline Z_Proposed_Method: \\
8.406975 + 11.2029i0.659248 + 5.8905i $0.659248+5.3971 \mathrm{i}$ \\
$0.659248+5.8905 \mathrm{i} 8.406975+11.2029 \mathrm{i} 0.659248+5.0263 \mathrm{i}$ \\
$0.659248+5.3971 \mathrm{i} 0.659248+5.0263 \mathrm{i} 8.406975+11.2029 \mathrm{i}$ \\
\hline Z_Deri: \\
$7.390430+7.6672 \mathrm{i} 0.780574+6.6913 \mathrm{i} 0.779930+5.6789 \mathrm{i}$ \\
$0.780574+6.6913 \mathrm{i} 7.390430+7.6672 \mathrm{i} 0.778610+4.9185 \mathrm{i}$ \\
$0.779930+5.6789 \mathrm{i} 0.778610+4.9185 \mathrm{i} 7.390430+7.6672 \mathrm{i}$ \\
\hline Z_Alvarado: \\
$7.657390+6.6245 \mathrm{i} 0.751964+6.6729 \mathrm{i} 0.751339+5.6606 \mathrm{i}$ \\
$0.751964+6.6729 \mathrm{i} 7.657390+6.6245 \mathrm{i} 0.750059+4.9007 \mathrm{i}$ \\
$0.751339+5.6606 \mathrm{i} 0.750059+4.9007 \mathrm{i} 7.657390+6.6245 \mathrm{i}$ \\
\hline Z_Noda: \\
$7.106818+7.1219 \mathrm{i} 0.114285+6.6749 \mathrm{i} 0.114175+5.6628 \mathrm{i}$ \\
$0.114285+6.6749 \mathrm{i} 7.106818+7.1219 \mathrm{i} 0.113949+4.9031 \mathrm{i}$ \\
$0.114175+5.6628 \mathrm{i} 0.113949+4.9031 \mathrm{i} 7.106818+7.1219 \mathrm{i}$ \\
\hline Z_Pizzaro: \\
$7.338788+9.5015 \mathrm{i} 0.780574+6.6913 \mathrm{i} 0.779930+5.6789 \mathrm{i}$ \\
$0.780574+6.6913 \mathrm{i} 7.338788+9.5015 \mathrm{i} 0.778610+4.9185 \mathrm{i}$ \\
$0.779930+5.6789 \mathrm{i} 0.778610+4.9185 \mathrm{i} 7.338788+9.5015 \mathrm{i}$ \\
\hline Z_Dubanton: \\
$7.479160+8.1232 \mathrm{i} 0.759264+6.5782 \mathrm{i} 0.754280+5.6409 \mathrm{i}$ \\
$0.759264+6.5782 \mathrm{i} 7.479160+8.1232 \mathrm{i} 0.756509+4.9735 \mathrm{i}$ \\
$0.754280+5.6409 \mathrm{i} 0.756509+4.9735 \mathrm{i} \quad 7.479160+8.1232 \mathrm{i}$ \\
\hline
\end{tabular}



KESHTKAR: MODELING AND CONTROLLING A HYBRID MULTI-AGENT BASED MICROGRID IN PRESENCE
OF DIFFERENT PHYSICAL AND CYBER COMPONENTS

Table 1.IV. Comparison of proposed methods for increasing length of long distribution line.

\begin{tabular}{|l|l|l|l|l|l|}
\hline Impedance (ohms) & Original & $5 \%$ & $15 \%$ & $20 \%$ & $28 \%$ \\
\hline \multirow{2}{*}{ Modified Carson } & $\begin{array}{l}8.6313+ \\
11.5019 \mathrm{i}\end{array}$ & $\begin{array}{l}9.0630 \\
+12.0771 \mathrm{i}\end{array}$ & $\begin{array}{l}9.9261 \\
+13.2273 \mathrm{i}\end{array}$ & $\begin{array}{l}10.3577 \\
+13.8024 \mathrm{i}\end{array}$ & $\begin{array}{l}11.0482 \\
+14.7225 \mathrm{i}\end{array}$ \\
\hline \multirow{2}{*}{ Proposed Method } & $\begin{array}{l}8.4069+ \\
11.2029 \mathrm{i}\end{array}$ & $\begin{array}{l}9.0012 \\
+12.0146 \mathrm{i}\end{array}$ & $\begin{array}{l}9.8953 \\
+13.1734 \mathrm{i}\end{array}$ & $\begin{array}{l}10.3154 \\
+13.8017 \mathrm{i}\end{array}$ & $\begin{array}{l}11.0121 \\
+14.7003 \mathrm{i}\end{array}$ \\
\hline \multirow{2}{*}{ Full Carson_3 term } & $\begin{array}{l}8.6170+ \\
\text { Full Carson_6 term }\end{array}$ & $\begin{array}{l}9.0479 \\
+11.5172 \mathrm{i}\end{array}$ & $\begin{array}{l}9.9096 \\
+12.0930 \mathrm{i}\end{array}$ & $\begin{array}{l}10.3404 \\
+13.2447 \mathrm{i}\end{array}$ & $\begin{array}{l}11.0298 \\
+13.8205 \mathrm{i}\end{array}$ \\
& $11.5171 \mathrm{i}$ & $\begin{array}{l}9.0479 \\
+12.0931 \mathrm{i}\end{array}$ & $\begin{array}{l}9.9097 \\
+13.2448 \mathrm{i}\end{array}$ & $\begin{array}{l}10.3405 \\
+13.8207 \mathrm{i}\end{array}$ & $\begin{array}{l}11.0301 \\
+14.7424 \mathrm{i}\end{array}$ \\
\hline
\end{tabular}

Table 1.V. Voltage, current and power flow results for a system with a long line and load

\begin{tabular}{|l|l|l|l|l|l|}
\hline \multirow{2}{*}{ Power flow outputs } & Bus 808 & Bus 812 & \multicolumn{2}{l|}{ Line 808-812 } & \multicolumn{2}{|c|}{$\begin{array}{l}\text { Reactive } \\
\text { power }\end{array}$} \\
\cline { 2 - 6 } & Voltage pu & Voltage pu & Current & $\begin{array}{l}\text { Active } \\
\text { power }\end{array}$ & 429.6 \\
\hline Full Carson_6 term & 0.99396 & 0.90247 & 110.417 & 664.8 & 429.6 \\
\hline Full Carson_3 term & 0.99396 & 0.90247 & 110.417 & 664.8 & 430.4 \\
\hline Modified Carson & 0.99393 & 0.90245 & 110.395 & 664.1 & 467.2 \\
\hline Proposed Method & 0.99640 & 0.91318 & 123.126 & 714.2 & 824.2 \\
\hline Deri & 1.02 & 0.92902 & 180.322 & 1070.3 & 737.8 \\
\hline Alvarado & 1.0214 & 0.95404 & 182.975 & 1131.5 & 771.5 \\
\hline Noda & 1.0201 & 0.92746 & 180.61 & 1108.9 & 917.3 \\
\hline Pizzaro & 1.0217 & 0.85981 & 182.629 & 947.2 & 776.2 \\
\hline Dubanton & 1.019 & 0.92672 & 178.93 & 986.0 & \\
\hline
\end{tabular}

Table 1.VI. Impedance for one configuration of 123 node system (ohm/mile)

\begin{tabular}{|lll|}
\hline Z_IEEE Test System: & & \\
$0.4576+1.0780 \mathrm{j}$ & $0.1560+0.5017 \mathrm{j}$ & $0.1535+0.3849 \mathrm{j}$ \\
$0.1560+0.5017 \mathrm{j}$ & $0.4666+1.0482 \mathrm{j}$ & $0.1580+0.4236 \mathrm{j}$ \\
$0.1535+0.3849 \mathrm{j}$ & $0.1580+0.4236 \mathrm{j}$ & $0.4615+1.0651 \mathrm{j}$ \\
\hline Z_Modified_Carson: & & \\
$0.457551+1.0780 \mathrm{i}$ & $0.155950+0.5016 \mathrm{i}$ & $0.153484+0.3849 \mathrm{i}$ \\
$0.155950+0.5016 \mathrm{i}$ & $0.466627+1.0481 \mathrm{i}$ & $0.158006+0.4236 \mathrm{i}$ \\
$0.153484+0.3849 \mathrm{i}$ & $0.158006+0.4236 \mathrm{i}$ & $0.461472+1.0650 \mathrm{i}$ \\
\hline Z_Proposed: & & \\
$0.457427+1.0774 \mathrm{i}$ & $0.155846+0.5005 \mathrm{i}$ & $0.153383+0.3837 \mathrm{i}$ \\
$0.155846+0.5005 \mathrm{i}$ & $0.466497+1.0471 \mathrm{i}$ & $0.157952+0.4229 \mathrm{i}$ \\
$0.153383+0.3837 \mathrm{i}$ & $0.157952+0.4229 \mathrm{i}$ & $0.461290+1.0644 \mathrm{i}$ \\
\hline Z_Full_Carson_3 terms: & \\
$0.457161+1.0790 \mathrm{i}$ & $0.155588+0.5026 \mathrm{i}$ & $0.153106+0.3859 \mathrm{i}$ \\
$0.155588+0.5026 \mathrm{i}$ & $0.466293+1.0491 \mathrm{i}$ & $0.157656+0.4246 \mathrm{i}$ \\
$0.153106+0.3859 \mathrm{i}$ & $0.157656+0.4246 \mathrm{i}$ & $0.461107+1.0660 \mathrm{i}$ \\
\hline Z_Full_Carson_6 terms: & \\
$0.457159+1.0790 \mathrm{i}$ & $0.155586+0.5026 \mathrm{i}$ & $0.153104+0.3859 \mathrm{i}$ \\
$0.155586+0.5026 \mathrm{i}$ & $0.466290+1.0491 \mathrm{i} 0.157653+0.4246 \mathrm{i}$ \\
$0.153104+0.3859 \mathrm{i}$ & $0.157653+0.4246 \mathrm{i}$ & $0.461105+1.0660$ \\
\hline
\end{tabular}

\section{III- Simulation Results}

In this section the effect of different impedance calculation methods is investigated by comparing impedances for a long distribution line. The long distribution line is selected from one of the longest lines in all IEEE Distribution Test Systems. Power flow solutions also compared for a system including this long line and a load. Comparisons are also made for impedance, power flow and short circuit calculations of IEEE 123 node system. Furthermore, the impact of changes in ground resistivity and frequency on impedance calculation results is analyzed.

\section{A. Impedance calculation and power flow analysis of long distribution line}

In this section, impedances calculated using methods discussed in section II are compared for a long distribution line from IEEE 34 node system. Power flow results using these impedances are obtained and compared for voltage magnitude, current and active power flow. 
Table 1.VII. Maximum Percentage error in power flow results of IEEE 123 node system by different impedance methods

\begin{tabular}{|l|l|l|l|l|}
\hline \multirow{2}{*}{ Results } & \multicolumn{4}{l|}{ Power flow analysis } \\
\cline { 2 - 5 } & voltage & current & active power & loss \\
\hline Full Carson_6term & 0.00993937 & 0.065539652 & 0.0235294118 & 0.0290 \\
\hline Full Carson_3term & 0.00993937 & 0.065539651 & 0.023529412 & 0.0291 \\
\hline Modified Carson & 0.339608 & 0.33458216 & 0.71090047 & 0.1240 \\
\hline Proposed Method & 0.0218709 & 0.0248382 & 0.0730714 & 0.0427 \\
\hline Deri & 1.961245 & 1.876326 & 3.035197 & 0.1818 \\
\hline Alvarado & 0.829104 & 0.766931 & 0.936519 & 0.1182 \\
\hline Noda & 1.831582 & 0.736194 & 1.917385 & 1.8166 \\
\hline Pizzaro & 2.183501 & 1.957928 & 1.057261 & 2.9522 \\
\hline Dubanton & 2.668132 & 2.063801 & 3.092869 & 0.3340 \\
\hline
\end{tabular}

Table 1.VIII. Maximum Percentage error in faults analysis results by different impedance methods

\begin{tabular}{|l|l|l|l|c|}
\hline \multirow{2}{*}{ Results } & \multicolumn{4}{l}{ Short circuit analysis } \\
\cline { 2 - 5 } & voltage & current & active power & loss \\
\hline Full Carson_6term & 0.026829 & 0.091264 & 0.051846 & 0.0394 \\
\hline Full Carson_3term & 0.026829 & 0.091263 & 0.051846 & 0.0397 \\
\hline Modified Carson & 0.319159 & 0.309318 & 0.693751 & 0.0847 \\
\hline Proposed Method & 0.0900148 & 0.101482 & 0.0720058 & 0.0438 \\
\hline Deri & 1.936501 & 1.896023 & 2.970351 & 1.8441 \\
\hline Alvarado & 0.840265 & 0.769252 & 1.236158 & 0.8904 \\
\hline Noda & 1.860014 & 0.696051 & 1.893401 & 1.7950 \\
\hline Pizzaro & 1.920024 & 1.829008 & 0.972201 & 1.2556 \\
\hline Dubanton & 2.438109 & 1.830917 & 2.780155 & 1.6841 \\
\hline
\end{tabular}

\section{A.1 Comparison of impedances of long distribution line}

The line between nodes 808 and 812 has a length of 37500 feet and is selected for the analysis as it is a long distribution line.

The conductor is 1/0 ACSR for phase and neutral with configuration as shown in Fig. 1.2. Impedances are calculated using the methods in section II and are as shown in Table 1.III.

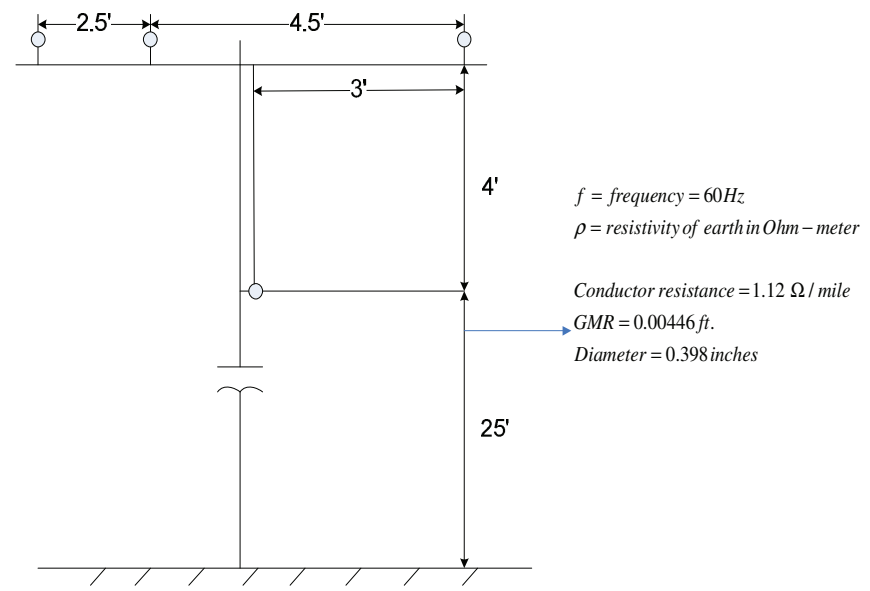

Fig. 1.2. Long distribution line configuration and parameters

The impedances obtained using various methods are compared for the maximum \% error in impedance elements with reference to numerical integration method, introduced in section II.C, as shown in configuration 1 of Table 1.II. It is observed that the proposed method has least error in calculation of real term of impedances whereas Noda's method has least error in calculation of imaginary term of impedances. Totally, the impedance error of the proposed method is less than other approximate methods.

It is concluded that proposed method can be a good replacement for most of the approximation methods. As it can be seen from Table 1.III, there is little difference in results of Carson's methods. By increasing the length of this long line we can distinguish the difference between these methods. These errors can increase based on the length of the line and can affect our impedance calculation results. The results for one of the self-impedances of the three-phase line $\left(\mathrm{Z}_{11}\right)$ are shown in Table 1.IV.

\section{A.2 Power flow analysis of long distribution line}

A $115 \mathrm{KW}$ load is added to the line at node 812. Using the impedances calculated in section A.1, power flow results are obtained. The results obtained for per unit voltages, currents and power flows at the two nodes where the line is connected are shown in Table 1.V. The results of proposed method are closer to Full Carson method than other approximate methods. It can also be seen from Table 1.V that the various forms of Carson's equations provide similar results.

\section{B. Impedance calculation, power flow and short circuit analysis of 123 node distribution system}

In this section, impedances calculated using methods discussed in section II are compared for IEEE 123 node system. Power flow and short circuit results using these impedances are obtained and compared for voltage magnitude, current and active power flow. 


\section{KESHTKAR: MODELING AND CONTROLLING A HYBRID MULTI-AGENT BASED MICROGRID IN PRESENCE OF DIFFERENT PHYSICAL AND CYBER COMPONENTS \\ B.1 Comparison of impedance of 123 node distribution system}

One of the configurations of IEEE 123 node system is selected for impedance comparisons. The conductor is 336,400 26/7 ACSR for phase and 4/0 6/1 ACSR neutral with configuration as shown in Fig. 1.2. The parameters of this line are presented in equation (1.54). Impedances are calculated using the methods in section II and are as shown in Table 1.VI.

$$
\begin{aligned}
& \text { phase }: \quad \text { Conductor resistance }=0.306 \Omega / \text { mile } \\
& \text { GMR }=0.0244 \mathrm{ft} . \quad \text { Diameter }=0.721 \text { inches } \\
& \text { neutral }: \text { Conductor resistance }=0.592 \Omega / \text { mile } \\
& \text { GMR }=0.00814 \mathrm{ft} . \quad \text { Diameter }=0.563 \text { inches }
\end{aligned}
$$

The impedances obtained using various methods are compared for the maximum \% error in impedance elements with reference to numerical integration method, introduced in section II.C, as shown in configuration 2 of Table 1.II. It is observed that proposed method has least error in calculation of real term of impedances whereas Noda's method has least error in calculation of imaginary term of impedances. Totally, the impedance error of proposed method is less than other approximate methods.

It can also be seen from Table 1.II that the errors in impedances for one of the configurations of IEEE 123 node system are less than the errors in impedances of long distribution line because of the increase in impedance due to the length of the line. The long distribution feeder shows the effects of only impedances on the errors however the 123 node system may introduce errors due to other components in the system as well.

\section{B.2 Power flow analysis of 123 node distribution power system}

Using the impedances calculated in section B.1, power flow results are obtained for per unit voltages, currents and power flows as shown in Table 1.VII. Fig. 1.3 plots the voltages at selected nodes and Fig. 1.4 plots power flow in selected lines. The results of proposed method are closer to Full Carson method than other approximate methods. It can also be seen from Table 1.VII that the various forms of Carson's equations provide similar results.

\section{B.3 Short circuit analysis of 123 node distribution power system}

Differences in voltages and currents calculated using different methods as seen in section B.2 can cause breaker misoperations. This section presents results for short circuit analysis of 123 node system.

Using the impedances calculated in section B.1, short circuit results are obtained for per unit voltages, currents and power flows as shown in Table 1.VIII. The results of proposed method are closer to Full Carson method than other approximate methods. It can also be seen from Table 1.VIII that the various forms of Carson's equation provide similar results. Since the protection settings are based on short circuit results, it is critical to use an accurate method for impedance modeling as it can result in considerable

differences as seen in Table 1.VIII.

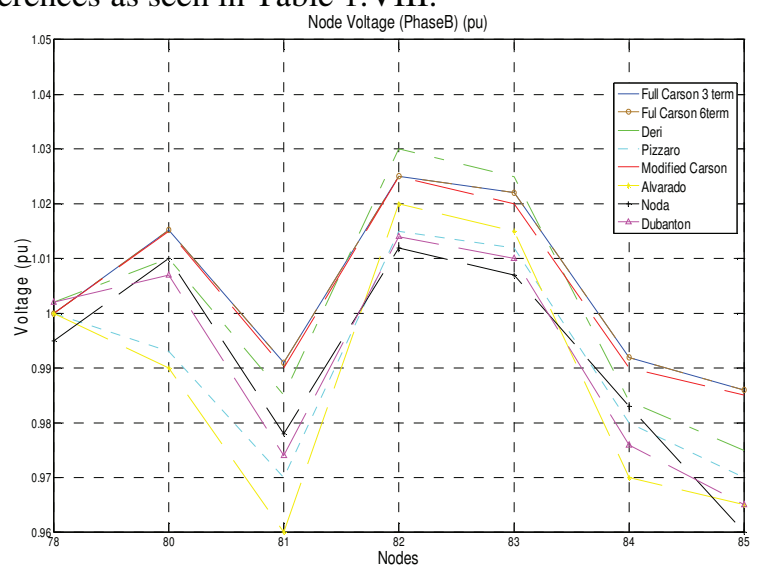

Fig. 1.3. Voltage (pu) for selected IEEE 123 node system using different methods

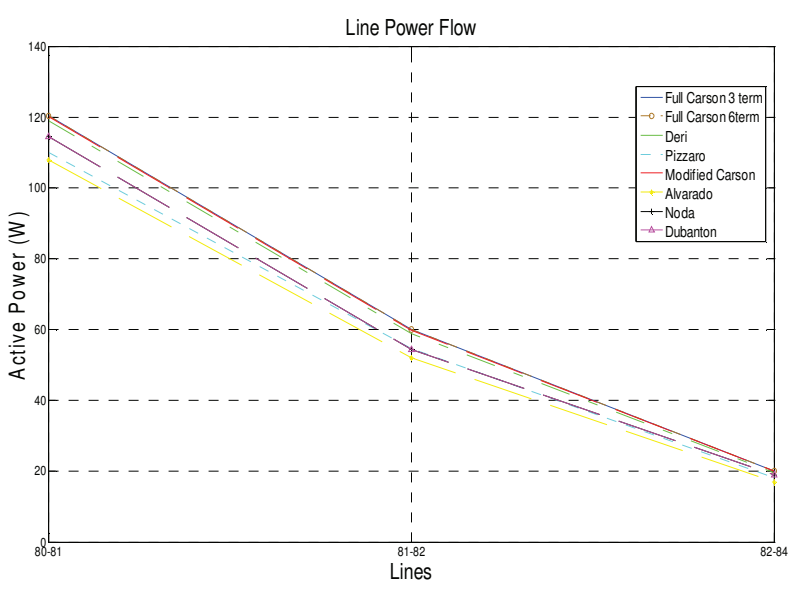

Fig. 1.4. Active power (W) for selected IEEE 123 node system lines using different methods 


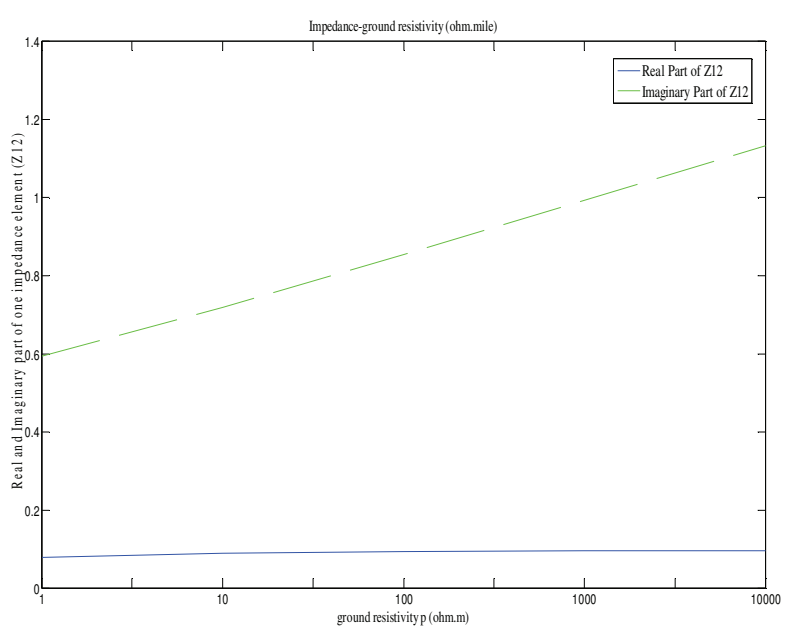

Fig. 1.5. Real and imaginary part of impedance respect to ground resistivity

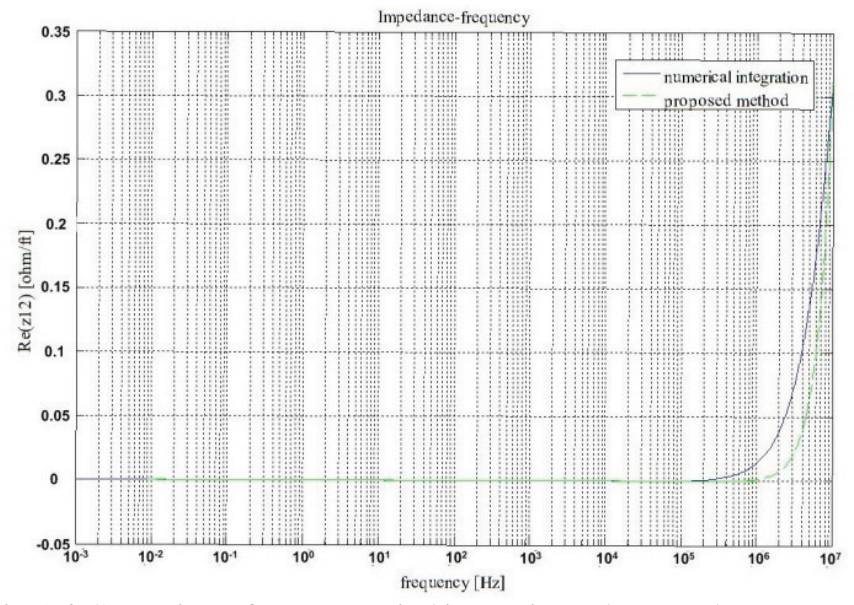

Fig. 1.6. Comparison of exact numerical integration and proposed approximation method of impedance calculations for different frequencies

Based on the observations on Table 1.VI-1.VIII, as it was expected from the discussion on math formulation in section II.C, the proposed method along with Carson's methods have the most accurate results in impedance calculation and consequently in power flow and short circuit analysis.

\section{Ground Resistivity Effect on Impedance Calculation Results}

In the methods discussed in section II the ground resistivity is considered as a constant (100 $\Omega \mathrm{m})$ while the impedances as seen by equations (1.1)-(1.4) can vary with ground resistivity. Ground resistivity is not constant and changes with the type of the ground and can vary from $1 \Omega \mathrm{m}$ for soil soaked with sea water to $10 \mathrm{k} \Omega \mathrm{m}$ for very rocky ground. Using six terms in Full Carson and considering the changing ground resistivity, the real and imaginary terms of one of the mutual impedances of the long distribution line considered in section III.A, are plotted as shown in Fig. 1.5. It is seen that if varying ground resistivity is considered there can be large difference in impedances especially the imaginary terms.

\section{Frequency Effect on Impedance Calculation Results}

In the methods discussed in section II the frequency is considered as a constant $(60 \mathrm{~Hz})$. In this section a numerical integration method described in section II.C is used to calculate the exact impedance values. A comparison has been made between proposed method and the exact results to study the impact of frequency on impedance calculations by approximation methods.

Change in impedance by frequency using proposed method as compared to numerical integration

Fig. 1.6 shows comparison of one mutual impedances of a line in section III.A using proposed method and numerical integration method for frequency ranges from $\mathrm{mHz}$ to $\mathrm{MHz}$.

The comparisons show impedances calculated by proposed method match closely with those calculated by numerical integration however differ slightly at higher frequencies.

\section{IV- CONClusions For PART 1}

In this part of the chapter, different methods for approximating the integral terms in Carson's equations have been investigated. The impedances are compared for long distribution line and IEEE 123 node system and percentage errors in impedances are calculated with Full Carson method as reference. The impedances are utilized for power flow analysis and tabulated for node voltages, currents and power flows. The impedances are also utilized for short circuit analysis and it is concluded that the variation in flows and voltages can lead to change in planning and designing aspects of protection devices such as fault clearing time of circuit breakers and impedance seen by distance relays. The results of proposed method are closer to the exact values by numerical 
KESHTKAR: MODELING AND CONTROLLING A HYBRID MULTI-AGENT BASED MICROGRID IN PRESENCE OF DIFFERENT PHYSICAL AND CYBER COMPONENTS

integration and Full Carson method than other approximate methods. It was also observed that the various forms of Carson's equation provide similar results. The proposed method is easier to implement as it uses infinite series with less complicated functions. Finally, it is observed and analyzed that the ground resistivity and frequency had pronounced effects on impedance calculations.

\section{Part 2. Analyzing Multi-Microgrid with Stochastic Uncertainties including Optimal PV Allocation}

\section{I- INTRODUCTION}

Electric energy is produced in large power plants and transmitted through High Voltage (HV) transmission systems to be distributed to consumers through Low Voltage (LV) distribution networks. Distribution system dynamics are changing with the siting of electricity generation closer to the loads and these are called Distributed Generation (DG) units [27]. These units have less environmental impact, easy siting, high efficiency, enhanced system reliability and security, improved power quality, lower operating costs due to peak shaving, and relieved transmission and distribution congestion.

However, depending on the location of DG units, some of the problems may be more pronounced and hence it is important to site the DG units to optimally exploit their potential. This part, therefore, develops algorithms to optimally place the distributed generator considering the changing demand and generation conditions over a day. With distributed generators the distribution network can work in isolation being separated from the feeder network to form a micro-grid without affecting the transmission grid's integrity. One of the DG technologies is Photovoltaic (PV), with penetrations increasing from hundreds of kWs to MWs in LV network. Due to these increasing penetrations in distribution systems, the utilities and planning engineers are increasingly interested in determining the best locations to place these units [28].

Much of the research work on PV allocation assumes a constant generation making the problem deterministic [29]-[35]. For example, in [35], analytical methods are presented to determine the optimal location of PV with constant generation to improve the power quality. In reality however the PV output has variations and hence an optimal location profiling the daily irradiation and energy production is necessary [36]. These units can be installed near load centers or at remote nodes to avoid large power transfers. In this part we develop an optimal PV location algorithm and analyze the effects of the multiple PV generations on total power loss of the system.

The organization of this part is as follows: Section II discusses about modelling of the distribution and microgrid system. PSO algorithm for optimal allocation of PV is discussed in Section III. Section IV describes the case studies and also presents the results of simulations. This research is concluded in Section V.

\section{II- MODELING AND PROBLEM FORMULATION}

In distribution systems, losses can increase operation cost and therefore it is essential to determine the optimal placement of the distributed generators to minimize the total losses. Here an optimal power flow type of problem is formulated and heuristic algorithm such as particle swarm optimization is selected. 


\subsection{Optimization algorithm}

In this part we will consider the placement of solar generation on $\mathrm{S} \subset \mathrm{N}$ nodes of distribution power system due to restriction imposed by distribution network operators. We assume that solar generation contributes majorly to the active power of the system thereby reducing the problem to minimization of active power losses. The methodology proposed here is described in three basic steps:

1) A constrained non-linear optimization problem is formulated to minimize the real power losses. Equality constraints related to distribution power flow equations and inequality constraints related to node voltage limits, generation capacity constraints and feeder current constraints are considered.

2) An intelligent computational technique like PSO is employed with reduced computational complexity due to reduction in search space from NPr to SPr where $r$ is the number of PV units to be placed. Since the order of units also matters a permutations calculator rather than a combinations calculator is employed.

3) PSO is combined with three phase distribution power flow computed using backward forward sweep algorithm while the PSO globally optimizes to find the optimal DG placements and the distribution power flow determines the constraints violations. In backward forward sweep method, Kirchhoff's Current Law and Kirchhoff's Voltage Law are used to compute the bus voltage from farthest node in the backward sweep. Then in forward sweep, downstream bus voltage is updated starting from source node. The procedure stops after the mismatch of the calculated and the specified voltages at the substation is less than a convergence tolerance.

4) If the optimal values of two consecutive iterations are same with all constraints satisfied the algorithm is deemed to have converged. If not, the process continues until the criterion is satisfied.

The minimization objective function is formulated as shown in (1.55).

$$
F=\sum_{i=1}^{N} \sum_{k=1}^{N} P_{\text {loss }_{i k}}
$$

Considering the conductor current $I_{i k}=\left(\frac{P_{i}+j Q_{i}}{V_{i}}\right)^{*}$ and current contribution from the solar generator as $I_{i}=\left(\frac{P_{i}}{V_{i}}\right)^{*}$, the losses of line ik can be expressed as in (1.56).

$$
\begin{aligned}
& P_{\text {loss }_{i k}}=3 .\left|I_{\text {loss }_{i k}}\right|^{2} \cdot R_{i k} \\
& =3 . \mid \begin{array}{l}
\left.\frac{P_{i} \cos \varphi_{i}+Q_{i} \sin \varphi_{i}}{\sqrt{3} V_{i}}-\frac{P_{D G_{i}}}{V_{i}^{*}}\right)^{2} \mid \\
+\left(\frac{Q_{k} \cos \varphi_{k}-P_{k} \sin \varphi_{k}}{\sqrt{3} V_{k}}-\frac{P_{D G_{k}}}{V_{k}^{*}}\right)^{2} \mid \cdot R_{i k}
\end{array}
\end{aligned}
$$

The losses over a period of one day continuously change due to changes in active power injections of the solar generation and changes in load consumption patterns. However, the location of PV once determined cannot change, so an optimal location should consider these variations in losses. The optimization algorithm is subjected to the following constraints.

(i) Generator rating constraint: Based on peak power generation, the minimum and maximum limits have been imposed on the generation capacity as

$$
P_{g_{i} \min } \leq P_{g_{i}} \leq P_{g_{i} \max }
$$

(ii) Voltage constraint: The optimal siting has to be obtained such that there are no bus voltages limit violations.

$$
V_{\text {min }} \leq V_{i} \leq V_{\text {max }}
$$


KESHTKAR: MODELING AND CONTROLLING A HYBRID MULTI-AGENT BASED MICROGRID IN PRESENCE OF DIFFERENT PHYSICAL AND CYBER COMPONENTS

(iii) Power balance constraint: The total power demand should be less than or equal to total power generation.

$$
\begin{gathered}
P_{d} \leq \sum_{i=1}^{r} P_{g_{i}} \\
Q_{d} \leq \sum_{i=1}^{r} Q_{g_{i}}
\end{gathered}
$$

(iv) Feeder current constraint: The feeder, current flowing through the feeder should be less than its thermal limit.

$$
I_{i k} \leq I_{t h i k}
$$

An unconstrained formulation considering both objectives and constraints from (1.55-1.60) is then given in (1.61). Typical operations constrain the voltage to be around the nominal node voltages of the distribution system whereas the lines have to be limited to their thermal values.

$$
\begin{aligned}
& F_{x}=\sum_{i=1}^{N} \sum_{k=1}^{N} P_{l_{\text {loss }}}+\sum_{i=1}^{r}\left\{\left\|P_{g_{i}}-P_{d}\right\|_{2}\right. \\
& \left.+\left\|Q_{g_{i}}-Q_{d}\right\|_{2}\right\}+\sum_{i=1}^{N}\left\|V_{i}-V_{i}^{N o m}\right\|_{2} \\
& +\sum_{i=1}^{N} \sum_{k=1}^{N}\left(I_{t h_{i k}}-I_{i k}\right)^{2}
\end{aligned}
$$

\subsection{Power Flow Equations}

The power balance constraints include the power flow equations that are solved iteratively and expressed as

$$
\begin{aligned}
& V^{i}(i)=a V^{k}(i)+b I^{k}(i) \\
& I^{i}(i)=c V^{k}(i)+d I^{k}(i)
\end{aligned}
$$

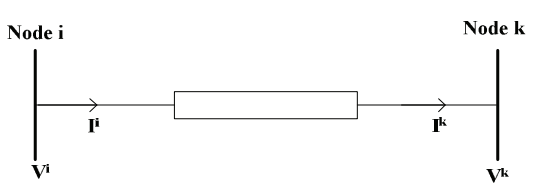

Fig. 1.7. Current flowing on line connecting node $i$ and $j$

The currents are those flowing on lines connecting nodes $\mathrm{i}$ and $\mathrm{j}$ as shown in Fig. 1.7 and a,b,c,d are matrix constants for the models of different components of the distribution network. Here loads are modelled as constant current injections, $I_{s}=\left(\frac{S_{s}(i)}{V_{s}(i)}\right)^{*}$, and distributed generations as negative active power loads. The generators are modelled using (1.63) that relates the voltage $u_{N}$ and the current $i_{N}$

$$
u_{N} \overline{i_{N}}=s_{N}\left|\frac{u_{N}}{U_{N}}\right|^{\eta_{N}}
$$

where $s_{N}$ is the nominal complex power and $\eta_{N}$ is a characteristic parameter of the node $\mathrm{N}$. The model (1.63) is called exponential model [37] and is widely adopted in the literature on power flow analysis [38]. Notice that $S_{N}$ is the complex power that the node would inject into the grid, if the voltage at its point of connection were the nominal voltage $U_{N}$. 


\subsection{Particle Swarm Optimization (PSO)}

The locations of the PV systems are optimized by Particle Swarm Optimization (PSO) Algorithm to minimize the total losses in the system presented in (1.61). PSO is a multi-agent search approach, which traces its evolution to the motion of a flock of birds searching for food [39]. It uses a number of particles that are called a swarm. Each particle traverses the search space searching for the global minimum (or maximum). In a PSO system, particles fly within a multidimensional search space. During flight, each particle sets its position based on its own experience and the experience of neighboring particles. Hence, it makes use of the best position encountered by itself and its neighbors. Similarly, the swarm direction and speed of a particle is determined by the history experience obtained by itself as well as a set of its neighboring particles [40-41].

Each particle is a representative of PV locations that are variables that affect the total losses in each iteration. Let us consider $\mathrm{p}$ and $\mathrm{s}$ as particle position and flight speed in a search space, respectively. The best position of a particle in each step is recorded and represented as $\mathrm{P}_{\text {best }}$. The best particle's index among all the particles in the group is considered as $\mathrm{G}_{\mathrm{best}}$. The convergence of PSO is ensured by use of a constriction function. Finally, the modified velocity and position of each particle can be calculated as shown in (1.64) and (1.65):

$$
\begin{gathered}
s_{d+1}=k^{*}\left(\gamma^{*} v_{d}+a c_{1}, \operatorname{rand}\left(0 *\left(P_{\text {best }}-P_{d}\right)+a c_{2} * \operatorname{rand}(0)\left(G_{\text {best }}-P_{d}\right)\right)\right. \\
P_{d+1}=P_{d}+s_{d+1}
\end{gathered}
$$

Here $d$ is the index of iteration, $P_{d}$ is the current particle's position at the $d$-th iteration, $s_{d}$ is the particle's speed of at $d$-th iteration, $\gamma$ is inertia weight factor, $\mathrm{ac}_{1}$ and $\mathrm{ac}_{2}$ are acceleration constants, $\mathrm{rand}()$ is a uniform random value in the range [0,1], and $\mathrm{k}$ is the constriction factor which is a function of $\mathrm{ac}_{1}$ and $\mathrm{ac}_{2}$ according to (1.66):

$$
k=\frac{2}{\mid 2-a c-\sqrt{a c_{2}-4 a c \mid}}
$$

Where $\mathrm{ac}=\mathrm{ac}_{1}+\mathrm{ac}_{2}$ and $\mathrm{ac}>4$. Appropriate choice of inertia weight, $\gamma$, makes a balance between global and local explorations. In general, $\gamma$ is calculated according to (1.67) [41]:

$$
\gamma=\gamma_{\max }-\frac{\gamma_{\max }-\gamma_{\min }}{\text { iter }_{\max }} \times \text { iter }
$$

Where iter $_{\max }$ is the maximum number of iterations, and iter is the number of the iterations up to current stage. The iterations continue until it reaches the iter ${ }_{\max }$ or the difference between the losses calculated by best particles of the last two iterations is less than a predefined threshold.

\section{III- Case Studies and Simulation Results}

The optimal PV locations are determined using the formulations discussed and the PSO technique. It is shown that losses are minimized under varying daily load consumptions. The PSO method described in section III was implemented in MATLAB programming language and the unbalanced power flow solution is obtained using OpenDSS.

Table 1.IX Total daily loss for different PV locations

\begin{tabular}{|c|c|c|c|c|c|c|c|c|c|c|}
\hline PV Bus & 808 & 814 & 816 & 828 & 832 & 834 & 840 & 848 & 860 & 890 \\
\hline Total daily Loss (MWh) & 3.8129 & 3.6524 & 3.6513 & 3.6182 & 3.4299 & 3.4457 & 3.4300 & 3.4294 & 3.4295 & 3.8688 \\
\hline
\end{tabular}

\subsection{Optimal PV allocation}

As a preliminary analysis a $0.5 \mathrm{MW}$ of PV generation is considered with an unknown optimal location that would result in minimum line losses. IEEE 34 node benchmark distribution system as shown in Fig. 1.9 is considered that is inherently unbalanced 
KESHTKAR: MODELING AND CONTROLLING A HYBRID MULTI-AGENT BASED MICROGRID IN PRESENCE OF DIFFERENT PHYSICAL AND CYBER COMPONENTS

with three phase cables and conductors and three phase, two phase and single phase loads. The characteristic curves of the PV are as shown in Fig. 1.8 (a)-(d).
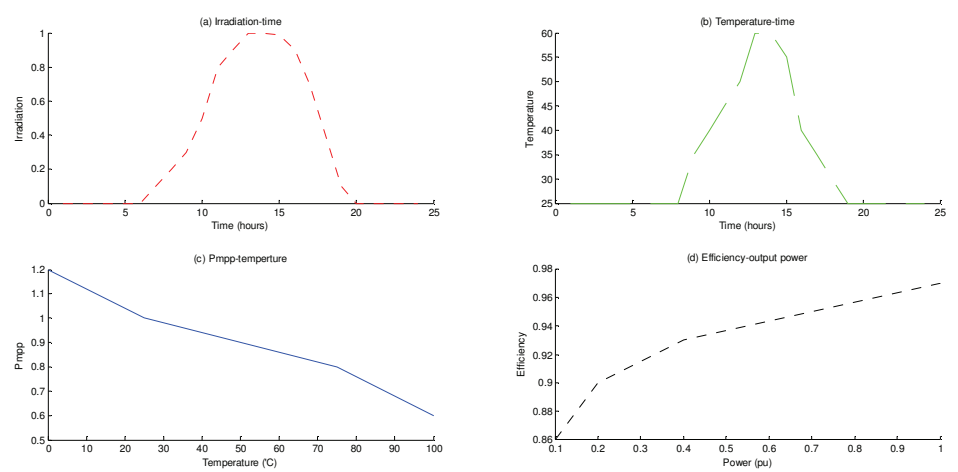

Fig. 1.8. PV Characteristic curves; (a) Irradiation-time, (b) Temperature-time, (c) $\mathrm{P}_{\mathrm{mp}}$-temperature, (d) Efficiency-power.

Initially losses are evaluated with single PV integration at node 848 of the IEEE 34 node system. Losses for an entire day are plotted as shown in Fig. 1.10 as the PV generation and loads vary throughout the day.

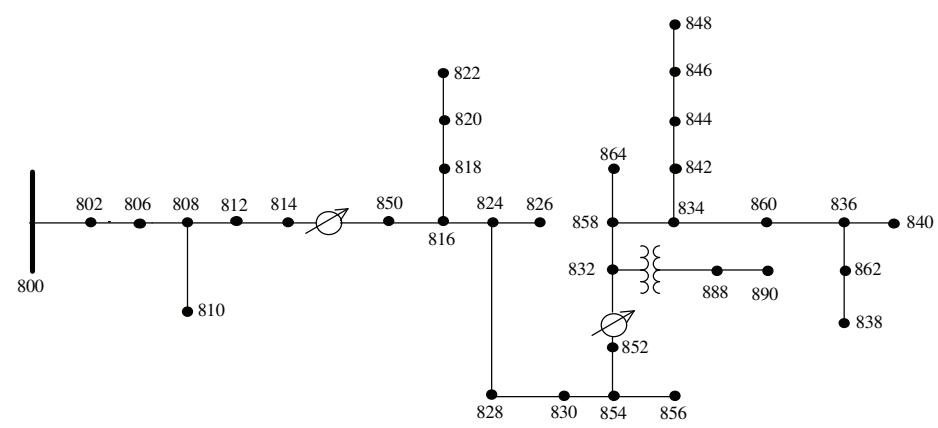

Fig. 1.9. IEEE 34 node test feeder configuration

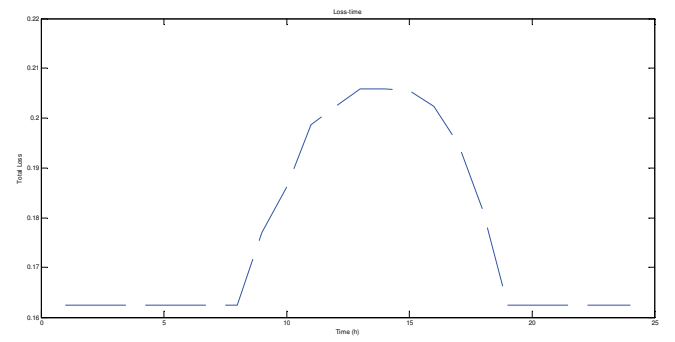

Fig. 1.10. Daily losses with PV placement at node 848.

The active power losses are low at night and in early morning time periods, when loading is less. However, when the loading increases and active power generations from PV source increases, the losses increase peaking from 15:00 - 16:00 hours. Table 1.IX summarizes the total daily losses with PV placements at different nodes. It is seen the best location is node 848 which is located further from the main grid and close to high-demand consumers. Similar results are obtained using the developed PSO algorithm and it is seen that losses are reduced by $29 \%$ as compared to no PV installation and $13 \%$ as compared to worst PV installation. 
Table 1.X Total daily loss for best and worst multi-PV locations

\begin{tabular}{|c|c|c|}
\hline & Best PV locations & Worst PV locations \\
\hline PV Nodes & $832,848,860$ & $808,814,890$ \\
\hline $\begin{array}{c}\text { Total daily Loss } \\
\text { (MWh) }\end{array}$ & 3.5620 & 3.8408 \\
\hline
\end{tabular}

\subsection{Optimal multi-PV allocation}

Optimal locations for three PVs are obtained for IEEE 34 node test feeder by optimization algorithm (PSO) to achieve the minimum total daily losses. As seen in Table 1.X the optimal node locations are 832, 848 and 860 . They show $8 \%$ improvement in losses as compared to the worst locations found heuristically.

\section{IV- CONCLUSIONS FOR PART 2}

In this section a method for determining the optimal placement of a PV system in distribution network based on daily power consumption/production fluctuations is described to minimize the total daily losses. The PSO optimization algorithm shows fast and accurate performance in calculating the optimal position of a PV system. Therefore, it can also serve as a tool in calculating the optimal placement of any number and kind of DG units with a specific daily production curve such as wind turbine systems, fuel cells, microturbines with a goal of optimizing distribution power system performance. The results show the effectiveness of the proposed approach in optimal placing of multiple DGs in a distribution power system to minimize the total loss.

\section{V- REFERENCES FOR CHAPTER 1}

[1] A. Deri, G. Tevan, A. Semlyen, A. Castanheira, "The Complex Ground Return Plane, a Simplified Model for Homogeneous and Multi-Layer Earth Return", IEEE Trans. on Power Apparatus and Systems, vol. PAS-I 00, August 1981, pp. 3686-3693.

[2] H W Dommel, "Overhead Line Parameters from Handbook Formulas and Computer Programs," IEEE Transactions on Power Apparatus and Systems, vol PAS-104, no 2, February 1985,pp. 366-372.

[3] H. Dwight, "Proximity Effect in Wires and Thin Tubes," IEEE Transactions, vol 42, 1923, pp 850-859.

[4] A P SakisMeliopoulos, Power System Grounding and Transients An Introducbon, New York, NY Marcel Dekker Inc , 1988.

[5] J R Carson "Wave Propagation in Overhead Wires with Ground Return," System Technical Journal, vol 5, October 1926,pp. 539-554.

[6] Adam Semlyen. "Accuracy Limits in the Computed Transients on Overhead Lines Due to InaccurateGround Return Modeling”, IEEE Trans. on Power Delivery, Vol. 17, No. 3, July 2002.

[7] P. Pettersson, "Image representation of wave propagation on wires above, on and under ground," IEEE Trans. on Power Del., vol. 9, no. 2, pp. 1049-1055, Apr. 1994.

[8] F. Pollaczek, "Sur le champ produit par un simple infiniment long parcouru par un altematif", Reme Gen. Elec, 1931, 29 , PP. $851-867$.

[9] W.H. Wise, "Effect of Ground Permeability on Ground Return Circuits", Bell Syst. Tech. J., 1931, vol.10, pp.472-484.

[10] L.M. Wedepohl, D.J. Wilcox, "Transit analysis of underground power-transmission systems", Proc. IEE, 1973, 120, pp. $253-260$.

[11] L. M. Wedepohl, "Application of Matrix Methods to the Solution of Travelling-Wave Phenomena in Polyphase Systems," Proc. IEE, vol. 110, December 1963, pp. 2200-2212

[12] R. H. Galloway, W. B. Shorrocks and L. M. Wedepohl, "Calculation of Electrical Parameters for Short and Long Polyphase Transmission Lines," Proc /E€, vol. 11 1, December 1964,

[13] L. M. Wedepohl and D. J. Wilcox, "Transient Analysis of Underground Power-Transmission Systems System-Model and Wave-Propagation Characteristics", Proc. IEE, vol. 120, February 1973,

[14] L. M. Wedepohl, H. V. Nguyen and G. D. Irwin, "Frequency-Dependent Transformation Matrices for Untransposed Transmission Lines using NewtonRaphson Method," IEEE Trans. on Power Systems, vol. PWRS-11, August 1996, pp. 1538-1546.

[15] O. Saad, G. Gaba, M. Giroux, “A Closed-Form Approximation for Ground Return Impedance of Underground Cables”, IEEE Trans. on Power Delivery, vol. 11, No. 3, July 1996.

[16] C. Gary, "Approche Complete de la Propagation Multifilaire en Haute Frequence par Utilisation des Matrices Complexes", EDF Bulletin de la Direction des Etudes et Recherches-Serie B, No. 3/4, 1976, pp. 5-20.

[17] E. Clarks,"Circuit Analysis of A.C. Power Systems", Vol. 1, John Wiley, New York, 1943. 
KESHTKAR: MODELING AND CONTROLLING A HYBRID MULTI-AGENT BASED MICROGRID IN PRESENCE

OF DIFFERENT PHYSICAL AND CYBER COMPONENTS

[18] G.O. Calabrese, "Symmetrical Components", Ronald Press, NY, 1959.

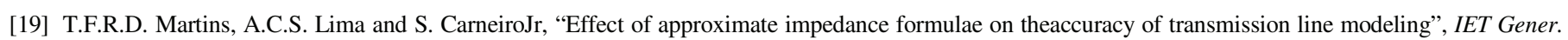
Transm. Distrib., 2007, 1, (4), pp. 534-539.

[20] W. H. Kersting, and R. K. Green, “Application of Carson's Equation to the Steady-State Analysis of Distribution Feeders”, IEEE, 2011.

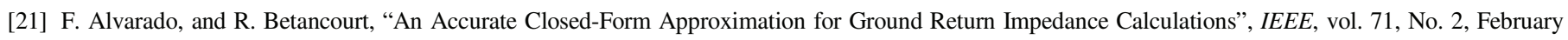
1983.

[22] T. Noda, “A Double Logarithmic Approximation of Carson's Ground-Return Impedance”, IEEE Trans. on Power Delivery, Vol.21, 2005.

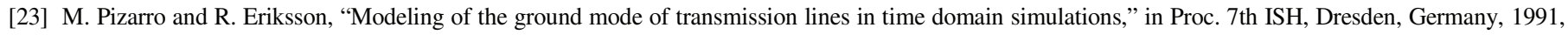
pp. 179-182.

[24] F.Pollaczek, “UberdasFeldeinerunendlichlangenwechselstromdurchflossenenEinfachleitung,” E.N.T., Band 3 (Heft 9), pp. 339-360, 1926.

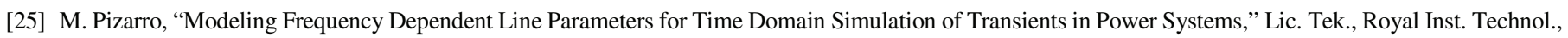
Stockholm, Sweden, 1991.

[26] W. Gander and W. Gautschi, "Adaptive quadrature-Revisited,” BITNumer. Math., vol. 40, pp. 84-101, 2000.

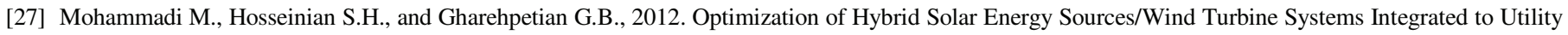
Grids as Microgrid (MG) Under Pool/Bilateral/Hybrid Electricity Market Using PSO. Solar Energy, vol. 86, pp. 112-125.

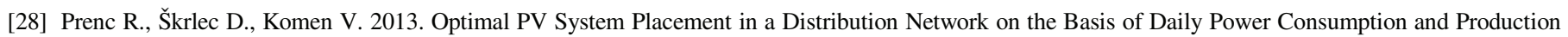
Fluctuation EuroCon, Zagreb, Croatia.

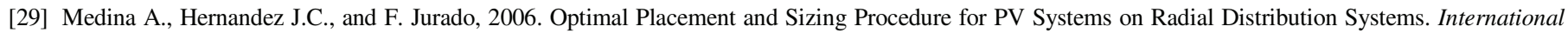
Conference on Power System Technology.

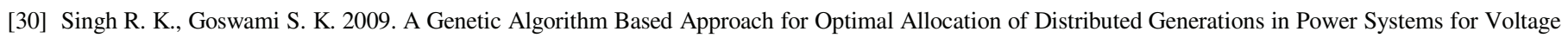
Sensitive Loads. ARPN Journal of Engineering and Applied Sciences.

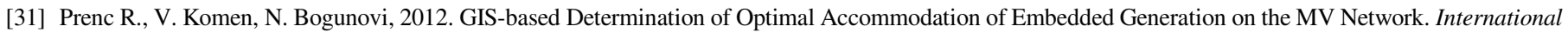
Journal of Communications Antenna and Propagation.

[32] Costa P. M., M. A. Matos, 2004. Loss Allocation in Distribution Networks with Embedded Generation. IEEE Transaction on Power System.

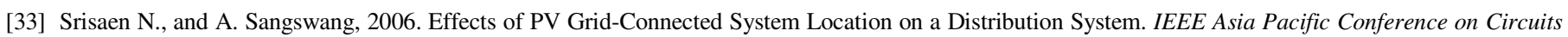
and Systems.

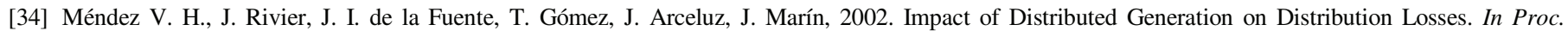
Mediterranean Power, Athens, Greece.

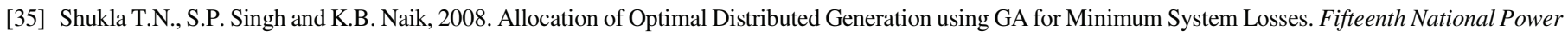
Systems Conference (NPSC), IIT Bombay.

[36] Ackermann T., G. Andersson, L. Söder, 2001. Distributed generation: a definition. Electric Power Systems Research, 2001.

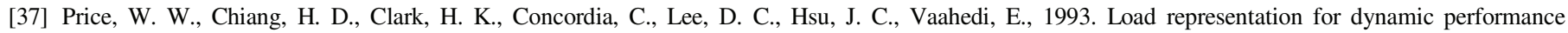
analysis. IEEE Transactions on Power Systems, 8(2), 472-482.

[38] Haque M. H., 1996. Load flow solution of distribution systems with voltage dependent load models. Elect. Pow. Syst. Res., vol. 36, pp. 151-156.

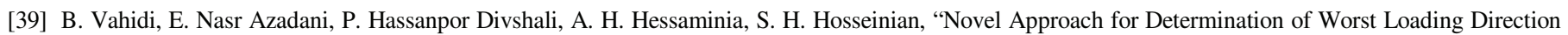
and Fast Prediction of Stability Margin in Power Systems", Simulation, Vol. 86, No. 1, 2010.

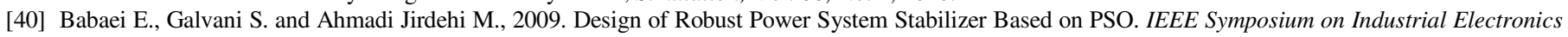
and Applications, Malaysia.

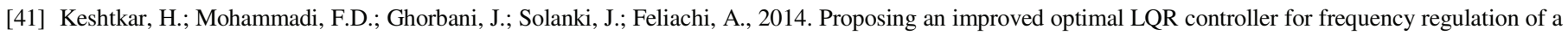
smart microgrid in case of cyber intrusions. IEEE 27th Canadian Conference on Electrical and Computer Engineering (CCECE).

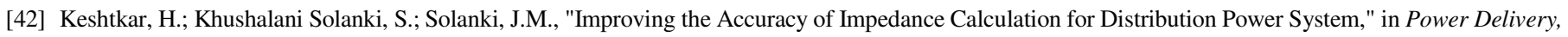
IEEE Transactions on , vol.29, no.2, pp.570-579, April 2014 


\section{Chapter 2}

\section{Novel Frequency Control and Defense Strategy against Cyber Intrusions in a Centralized Multi-agent based Microgrid}




\section{KESHTKAR: MODELING AND CONTROLLING A HYBRID MULTI-AGENT BASED MICROGRID IN PRESENCE OF DIFFERENT PHYSICAL AND CYBER COMPONENTS}

Previously, the challenges in modeling different parts of a distribution power system was discussed. In this chapter the distribution power system is equipped with different small scale energy sources to model a smart hybrid microgrid in order to implement novel control strategies against existing disturbances in the power system. The study starts with modeling different load and generating sources and continues with implementing control methods in a centralized multi-agent structure for frequency regulations against cyber intrusions on the communication signals. Simulation results show effectiveness of the proposed control and defense strategy in mitigating the frequency oscillations and improving the reliability and stability of the microgrid system.

\section{I- INTRODUCTION}

\section{- Literature Survey}

\section{A. Microgrid \& Frequency Control}

The distribution power systems having small scale energy sources such as Distributed Generators (DGs) and RES are called as microgrids [1]. These small-capacity sources are mostly connected at the distribution level. So by shortage in line length, transmission and distribution losses are reduces as the sources are close to the load. The main aim of microgrid operation control is to reliably serve the loads while maintaining the security and stability of the system. Oscillatory stability, especially while rapid changes in load and generation are faced, is one of the most important problems involved in these criteria. Under rapid changes of load and generation system frequency can deviate considerably from the normal operating state leading to stability issues especially when the frequency of load variation is near the inter-area oscillation modes $(0.2-0.8 \mathrm{~Hz})$.

Microgrids mostly operate in an on-grid mode. However, contingencies such as fault, voltage sag and large frequency oscillations in the main grid may force the active distribution network to become off the grid and operate in an isolated mode [2]. During this isolation there will be change in power output of the controllable microsources, which are used to regulate the power flows to have a stable operation related to power balance and system frequency. One solution is to have a diesel generator unit as a DG alternator to fit the mismatch between the power produced by the RESs and loads [3].

The frequency instability under normal operation, contingencies, and islanding results in need of Load Frequency Control (LFC) [4], where power system frequency is controlled to nominal value. The main effect of LFC is to keep the system frequency and steady state of the system at desired values and to ensure some equity in the participation of the various generation units to the control action.

As a result of small and sudden disturbances of power demands in distribution power systems, deviation in frequency and tieline power flow is created. As a matter of fact, a small load change results in changes in the generator torque which causes imbalance between electrical and mechanical torque and hence change in output speed and frequency. Furthermore in recent years, distributed and renewable generation penetrated in micro grid beside demand side response method in Smart Grids environment has increased the importance of frequency stabilization. As a result, different control methods associated with power flow control are proposed in different literatures [5-6].

Load frequency control in microgrids with nominal frequency of $50 \mathrm{~Hz}$ is designed to maintain frequency within 49.9 and 50.1 in normal condition by controlling tie-line flows and generator load sharing [7-9]. The control strategy should damp the frequency oscillation in steady state and minimize them in transient state while maintaining stability. Frequency in rated and 
acceptable range, to load shedding among generators, and to control tie-line power flow are the main application of LFC systems. LFC in various distribution power systems with different generation units and energy storage systems have been analyzed in recent years [10-14].

\section{B. Microgrids and RES, DG, ESS}

Today one of the concerns of the world is to reduce green-house gas emission from the thermal power plants, as cutting down emissions from transport and heating sector may not be probable in the near future. To reduce pollution from power sources, the world is now looking for usage of renewable energy sources (RESs) [15-17]. Recently, micro grid is proliferated with Distributed Generation (DG) units and renewable energy sources. Some renewable resources such as wind generation units have a few deviations in their outputs. Fast-response Energy Storage Systems (ESSs) are used for damping oscillations in frequency of distribution power systems [18].

Fast-response ESSs can effectively damp electromechanical oscillations in a power system, because they provide storage capacity in addition to the kinetic energy of the generator rotors which can share sudden changes in power requirement [19]. The problems like low discharge rate, increased time required for power flow reversal and maintenance requirements have led to the evolution of HVDC links and ESSs such as BESS and Superconducting Magnetic Energy Storage (SMES) devices for their applications as load frequency stabilizers. The application of SMES for load frequency control is discussed in [20]. Effectiveness of SMES as an ESS device in frequency stabilization is investigated in [21].

\section{Microgrids and Microrurbine}

With growing power demand and increasing concern about the depletion of fossil fuel, use of distributed generation (DG) resources such as solar photovoltaic, wind turbines, fuel cell, microturbine, etc are spreading and are going to have greater commercial and technical importance across the world [22-23].

DG sources could have positive impact on the operation of power systems to which they are connected. In Smart Grid environment, DG source is one of the main parts of the demand-side power system [24]. To study their operation and impact on power systems, proper modeling of DGs is needed. Improving the power system stability and reliability, providing the power supply economically, energy storage and environmental protective consideration increase the attention to DG [25].

High energy efficiency, low environmental impact, and the applicability as uninterruptible power supplies are some of the advantages of DG. It has several benefits to the customers, utilities, and the environment [26-30]. Distributed resources are widely used in Smart Grids and their control design is one of the main necessities of this application. Thus study of operation and control of distributed generators in power islands in Smart Grid environment is necessary. Without consideration of the technology and cost of the control system, several reliability and stability problems will appear.

Distributed generation can be used in renewable technologies such as wind turbine, photovoltaic or recent promising nonrenewable technologies such as microturbine and fuel cell. It is widely accepted that microturbine play an important role in power generation [25]. If it connects to power distribution systems, these generators will affect the dynamics of the system. The transient behavior of microturbine-based system can be assessed only if a detailed nonlinear dynamics model is used. Thus an accurate model of the microturbine is needed to analyze factors such as power quality like harmonics, voltage regulations when connected to distribution network [31].

Microturbine powered generators rating from $25 \mathrm{~kW}$ to $250 \mathrm{~kW}$ provide a variety of opportunities to meet the applications of distributed power systems [32-36]. The applications of MT in the network are including peak saving, co-generation, remote power and premium power. It is composed of compressor, recuperator, control system and drive system. 
KESHTKAR: MODELING AND CONTROLLING A HYBRID MULTI-AGENT BASED MICROGRID IN PRESENCE OF DIFFERENT PHYSICAL AND CYBER COMPONENTS

As study about distributed generation require appropriate and accurate modeling of power distribution system and the mathematical model of MT is complex and very strong nonlinear, and there is strong interference due to existing of high frequency converter, it is vital to find the parameters of the controllers exactly. One part of dissertation, therefore, proposes a method to design suitable control parameters of speed governor of MT system. Then this model has been added to a hybrid Microgrid to study its participation in frequency control purpose.

\section{Microgrid and PHEV}

Plug-in hybrid electric vehicle (PHEV) technology is a promising solution that can lead to decrease greenhouse gas emissions, air pollution in urban areas and totally it can be considered as environmentally clean. Recent studies have shown the effect of the PHEVs on the grid. In literatures, vehicle to grid (V2G) is considered to evaluate the amount of real power transmitted between vehicle and grid in charge and discharge modes [37-39].

Recently PHEVs are being emphasized as a means of transportation to achieve fossil fuel independence and solve the problem of greenhouse emission. PHEVs can be connected to grid for charging and can operate independent of grid for transportation needs. When connected to the grid the PHEVs can charge or discharge to act as demand or generation [40-43].

However, there are several obstacles to the proliferation of PHEV including short driving range and long battery charging time. The increasing number of PHEVs is expected to add an additional $18 \%$ load to existing power grids and this may impact the grid reliability due to less predictable overloads [44-45]. These additional loading will occur during afternoon and evening hours when people arrive home from their daily work commutes and connect their vehicles to charging station which will be located at residences rather than businesses. This additional loading will affect the dynamic aspects of microgrid. For instance, extra load comparing to generation can drop the frequency of the system instantly.

Since no simulation work has been carried out on a microgrid to actually observe how different PHEV penetration levels will impact the dynamic aspects of the electrical system, the grid will be used for analysis in order to obtain power flow data and frequency response for several loading variation due to various PHEV charging scenarios and wind speed deviation. Additionally, the effect of controlling PHEV as an Energy Storage of the system for frequency control can also be investigated. These scenarios are conducted with variations in the number of total vehicles.

Furthermore, the behavior of PHEV is in a stochastic manner. To study its behavior needs information on vehicles' driving behavior, such as departure and arrival time, parking location and energy consumption, none of which can be perfectly forecasted. One solution is that different possible patterns of PHEV behavior will be modeled with random scenarios, and modeling individual driving behavior with Markov or non-Markov chains [46-47].

There are different types of random distributions [48]:

1) First type is the random number generators such as Uniform distribution, Zero-one distribution, Binomial distribution, Normal distribution, Exponential distribution, Geometric distribution, Poisson distribution, Stationary distribution, etc.

2) Second type is random processes such as random walk, Brownian motion, Poisson processes, Higher-dimensional versions of random processes, etc.

3) Third type is Markov processes such as Queuing systems, Birth-and-death processes, Branching processes.

These behaviors in the output of PHEVs are existed and influence different aspects of the power system. Therefore, PHEVs stochastic behavior modeling and their effect on the frequency control of microgrid are studied in the next sections of this dissertation. 


\section{E. Multi agent system and Cyber physical system}

By introducing multi-agent system in microgrid power systems and using different kinds of communication between the agents, the possibility of occurring cyber attacks is increased and investigated in literatures [49-52]. Different control approaches and defense strategies have been proposed in order to increase the resiliency of the system against these contingencies which usually happen on the measurement signals of the power system [53-56]. These kinds of works are considered as building module for future works in cyber-physical energy systems area in smart grids.

\section{- Chapter Content}

This Chapter is discussing about implementing frequency control of a centralized multi-agent based microgrid. This implementation is carried out step by step and starts from modeling the microgrid power system and analyzing the effect of different generation and demand devices. Then, implementation of a centralized multi agent system and novel frequency control is investigated in this power system. The resiliency of the model microgrid is tested and improved in presence of different cyber attack by proposing defense strategy based on detection and learning algorithm.

In the first part of the second chapter, the objective is to model and control the frequency oscillations of hybrid microgrid distribution power system including DGs, renewable resources, and ESSs due to sudden changes in load and in wind speed by optimal employment of FC, AE, WTG, DEG, various ESSs. The application of SMES as an ESS and three configurations of WTG and PV, is analyzed and compared with each other. A novel comparative assessment for deviation in frequency profile for these different configurations is made. The simulation model and results are introduced in the next sections.

In the second part of this chapter, the main aspect of the research is to use PHEV as loads/generations to analyze how the additional power demand from the PHEVs would affect the dynamic aspects of the microgrid. Firstly, this section reviews the modeling of the studied power system including wind generators, photovoltaic, diesel generators, PHEV. Moreover, modeling of the distribution power system, Load Frequency Control Problem in microgrids and the applied optimization algorithm to the problem are also presented in this section. The main aspect of this part of research is to use a microgrid system and the PHEV loads to analyze how the additional power demand from the PHEVs would affect the dynamic aspect of the system. In dynamic point of view, they can have both advantages and disadvantages for the system which they are using for. As an advantage, PHEVs can perform as energy storage for the system that their output can be useful in the case of deviation in system response. However, uncontrolled use of PHEVs can act as a load disturbance for the system and make the outputs more oscillatory [57]. Secondly, in order to model the stochastic behavior of the PHEVs different kinds of random distributions have been implemented and studied and four different types of these processes have been chosen to be modeled as stochastic behavior of PHEVs in the power system.

Third part of this chapter proposes a method to design suitable control parameters of speed governor of MT system. Then this model has been added to a hybrid Microgrid to study its participation in frequency control purpose.

In the last part of the chapter, the modeling of a smart microgrid is carried out along with a central control for frequency response. The communication between the power system part and the controllers is implemented using centralized multi-agent concept. The objective is to control the frequency oscillations of a multi-agent based smart microgrid system including DGs, renewable resources, and ESSs due to load changes and cyber attacks. The application of the proposed control methods including Optimal LQR and Adaptive Dynamic Programming in the modeled microgrid are also studied in presence of different cyber attacks including replay attack and data loss and compared with using conventional PI controllers. Detection and learning method as a defense strategy against the attacks on the modeled microgrid is also proposed and simulated. Particle Swarm Optimization algorithm is used to determine the control parameters for minimizing the frequency oscillations due to cyber intrusions. 
KESHTKAR: MODELING AND CONTROLLING A HYBRID MULTI-AGENT BASED MICROGRID IN PRESENCE OF DIFFERENT PHYSICAL AND CYBER COMPONENTS

The rest of this chapter is organized as follows. Next section deals with the modeling and system configuration, problem formulation for each section, control and optimization algorithms, and introducing case studies which is used in the simulation analysis section. Simulation results and analysis are shown in Section III. The chapter is concluded in the last section.

\section{II- Modeling And Problem Statement}

\section{LFC FOR HYBRID POWER GENERATION/ENERGY STORAGE MICROGRID SYSTEM}

\subsection{System Configuration and Simulation Model}

The hybrid power generation/energy storage system considered in this part is shown in Fig. 2.1. This system consists of wind generators, photovoltaic, diesel generators, fuel-cell generators, aqua electrolyzers, battery energy storage, and superconductive magnetic energy storage [58]. This microgrid will be used as the base for the next parts of this document for proposing different device modeling and control approaches. Power supplied to the load $\mathrm{P}_{\mathrm{s}}$ is the sum of output power from wind turbine generators $\mathrm{P}_{\mathrm{w}}$, diesel generators $\mathrm{P}_{\mathrm{g}}$, photovoltaic generation $\mathrm{P}_{\mathrm{pv}}$, fuel-cell generators $\mathrm{P}_{\mathrm{fc}}$, and negative input power to aqua electrolyzers $\mathrm{P}_{\mathrm{r}}$ given by

$$
P_{s}=P_{w}+P_{g}+P_{p v}+P_{f c}-P_{r}
$$

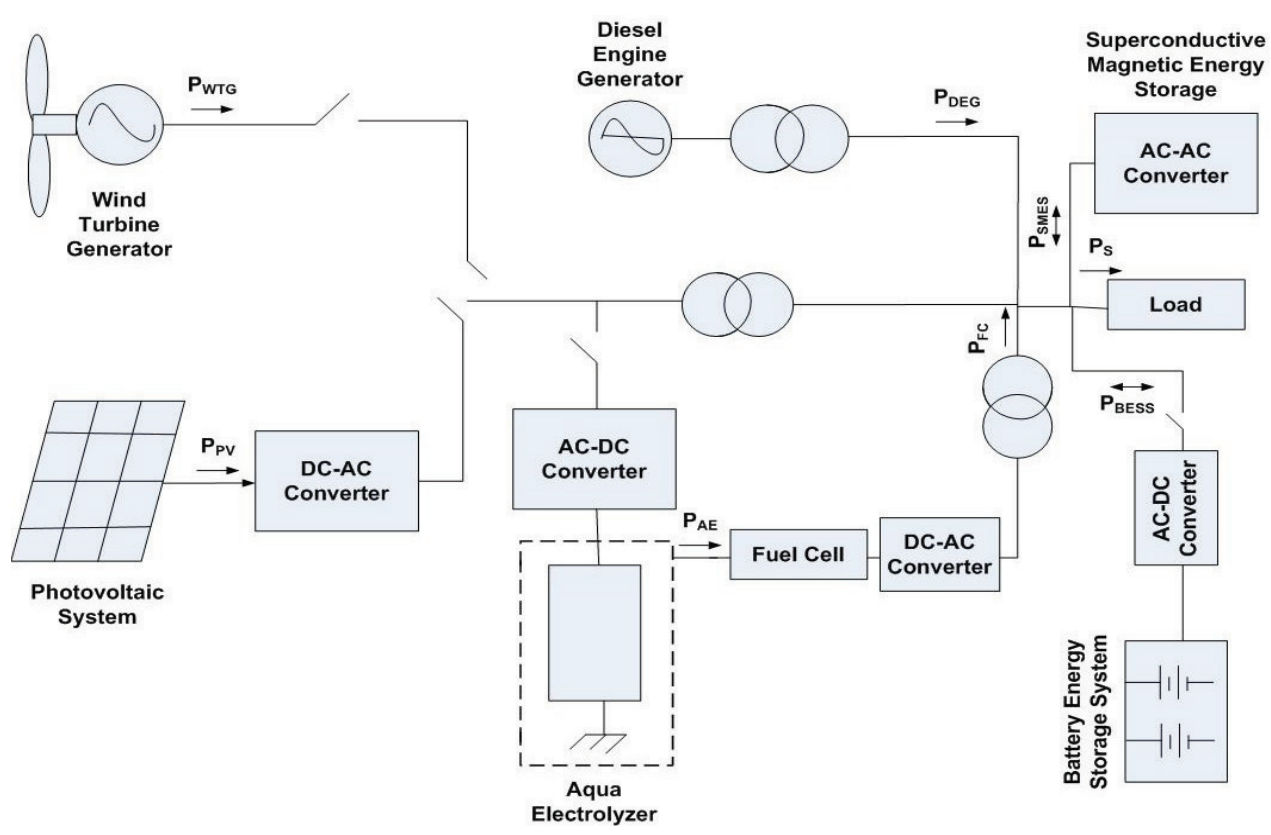

Fig. 2.1. Configuration of the model microgrid

The used devices are simulated in transfer function models [59]. The parameters of the considered system including generating units and microgrid system are shown in Table 2.I [58].

Table 2.I. Parameters of the microgrid system

\begin{tabular}{|c|c|c|c|}
\hline $\begin{array}{c}\text { Kae=1, } \\
\text { Tae=1 }\end{array}$ & $\begin{array}{c}\text { Kfc=1, } \\
\mathrm{Tfc}=4\end{array}$ & $\begin{array}{c}\mathrm{Kpv}=1, \\
\mathrm{Tpv}=1.8\end{array}$ & Kbess=1, Tbess=0.1 \\
\hline $\begin{array}{c}\mathrm{Kdeg}=1, \\
\mathrm{Tdeg}=2\end{array}$ & $\begin{array}{c}\mathrm{Kwtg}=1, \\
\mathrm{Twtg}=1.5\end{array}$ & $\begin{array}{c}\mathrm{M}=10, \\
\mathrm{D}=1\end{array}$ & $\begin{array}{r}\mathrm{Kn}=0.4, \mathrm{Khvdc}=0.005, \text { Thvdc }=0.7 \\
\text { Ksmes }=0.09, \mathrm{~T} \text { smes }=0.024, \\
\mathrm{~T} 1 \mathrm{smes}=0.645, \mathrm{~T} 2 \mathrm{smes}=0.25, \\
\mathrm{~T} 3 \mathrm{smes}=0.129, \mathrm{~T} 4 \mathrm{smes}=0.027\end{array}$ \\
\hline
\end{tabular}




\section{A. Controllable Sources Transfer Functions}

The transfer functions of governor and turbine of the diesel generator is used based on the transient response of these sources. So these functions relate frequency change and output power changes.

$$
G_{d g g}(s)=\frac{K_{d g g}}{\left(1+s T_{d g g}\right)} ; G_{d g t}(s)=\frac{K_{d g t}}{\left(1+s T_{d g t}\right)}
$$

Where $\mathrm{K}_{\mathrm{dgg}}, \mathrm{K}_{\mathrm{dgt}}$ are gain constant and $\mathrm{T}_{\mathrm{dgg}}, \mathrm{T}_{\mathrm{dgt}}$ are time constant of diesel generator governor and turbine, respectively. The transfer function which is used for fuel cell is also shown in below

$$
G_{f c}(s)=\frac{K_{f c}}{\left(1+s T_{f c}\right)}
$$

Where $\mathrm{K}_{\mathrm{fc}}, \mathrm{T}_{\mathrm{fc}}$ are gain and time constant of fuel cell. The transfer functions of aqua electrolyzer which shows the relation between input changes and output is shown in Eq. (2.4).

$$
G_{a e}(s)=\frac{K_{a e}}{\left(1+s T_{a e}\right)}
$$

Where $\mathrm{K}_{\mathrm{ae}}, \mathrm{T}_{\mathrm{ae}}$ are gain and time constant of aqua electrolyzer.

\section{B. Power system modeling:}

For modeling of the power system we have to come up with the relationship between the power mismatch deviation and related frequency changes. On the other hand, for a power system having one synchronous generator we can write the swing equation based on Eq. (2.5).

$$
\begin{aligned}
& \Delta f=\frac{f_{s y s}}{2 H s}\left[\Delta P_{G}-\Delta P_{e}\right] \\
& \text { where } \\
& P_{G}=P_{W}+P_{s}+P_{d g}+P_{f c}-P_{a e} \\
& \Delta P_{e}=\Delta P_{L}+D \Delta f
\end{aligned}
$$

where $\mathrm{P}_{\mathrm{W}}, \mathrm{P}_{\mathrm{s}}, \mathrm{P}_{\mathrm{dg}}, \mathrm{P}_{\mathrm{fc}}, \mathrm{P}_{\mathrm{ae}}$ are generating power by different generating units, $\mathrm{f}_{\mathrm{sys}}$ is the steady state frequency of the system, $\mathrm{D}$ is the load damping factor, and $\mathrm{H}$ is machine inertia. After some calculations, the transfer function model of the power system is

$$
G_{s y s}(s)=\frac{\Delta f}{\left(\Delta P_{G}-\Delta P_{L}\right)}=\frac{1}{\left[D+\left(\frac{2 H}{f_{s y s}}\right) s\right]}=\frac{K_{p s}}{1+s T_{p s}}
$$

Where $\mathrm{K}_{\mathrm{ps}}$ is $1 / \mathrm{D}$ and $\mathrm{T}_{\mathrm{ps}}$ is $2 \mathrm{H} /\left(\mathrm{Df}_{\mathrm{sys}}\right)$.

The parameters of the sources and power system model are obtained from [13]. This model is used in Section III of this chapter as the case study for simulation analysis.

\section{Dynamic Modeling, Control and Stability Analysis of Microturbine in a MICROGRID}

In this part modeling and controlling a MT in the microgrid system is investigated. Part 2.1 reviews the modeling of a MT and its control schemes. Moreover, modeling of PM generator, power conditioning and voltage control system are also presented in this part. Then, the problem corresponding to the transient response of the speed and fuel consumption of MT in 24 hours of daily load variations is defined. Next, the multi-objective optimization procedure including normalizing technique and Differential 

KESHTKAR: MODELING AND CONTROLLING A HYBRID MULTI-AGENT BASED MICROGRID IN PRESENCE
OF DIFFERENT PHYSICAL AND CYBER COMPONENTS

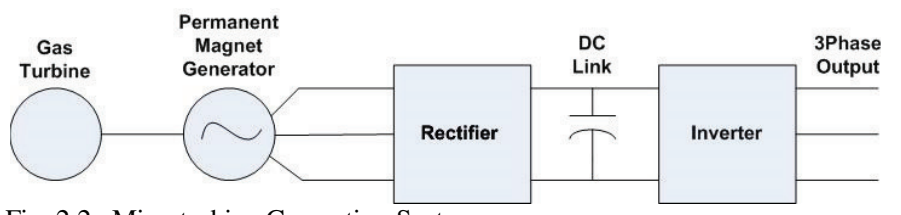

Fig. 2.2. Microturbine Generation System

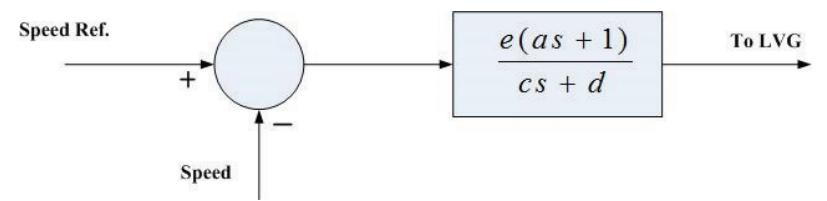

Fig. 2.3. Speed Control System

Evolutionary algorithm for speed governor problem is presented.

Case studies and simulations are performed in the simulation results section (section III of this chapter) which shows the effectiveness of the proposed method for modeling of MT as well as for the purpose of frequency control in a Microgrid.

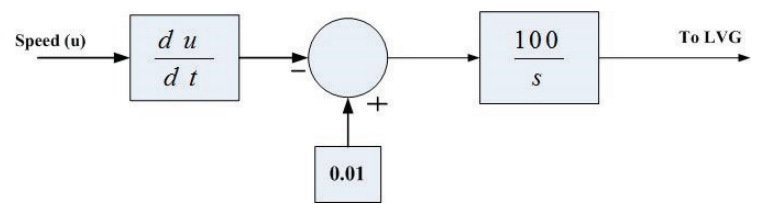

Fig. 2.4. Acceleration Control System

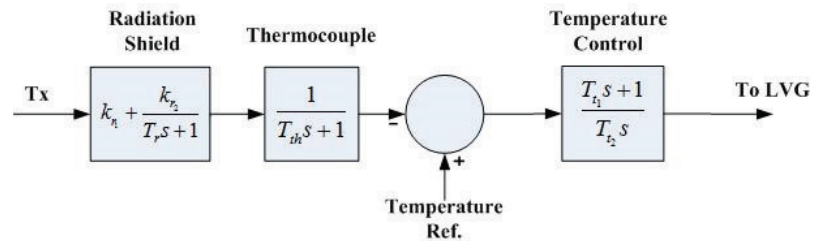

Fig. 2.5. Temperature Control System

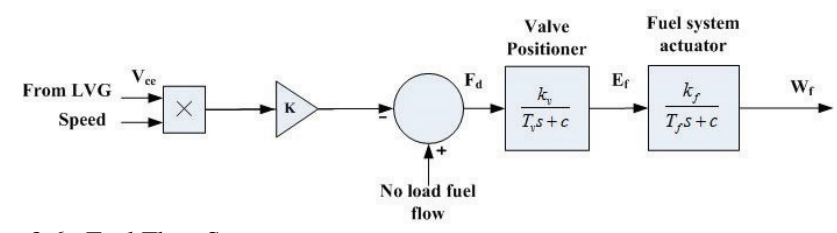

Fig. 2.6. Fuel Flow System

\subsection{Microturbine system and permanent magnet generator modeling}

In this part, the modeling of the MT is discussed. It is assumed that the system is operating under normal operating conditions. Thus fast dynamics of the microturbine including start-up, shut down, internal faults and loss of power, are neglected.

There are two basic models of MT design. One of them is a single-shaft design that the compressor, microturbine and permanent magnet generator are mounted on one shaft. In this design the frequency of the generator is very high. Rectifier and inverter are essential for changing the high frequency voltage to normal 50 or $60 \mathrm{~Hz}$ voltage. Another model of MT design is a split or twin shaft design. The compressor and the turbine are mounted on one shaft and the power turbine is mounted on the same shaft as the generator. The power turbine is rotating at $3600 \mathrm{rpm}$ and the generator connects it via a gearbox. The power inverters are not necessary in this design. The first aforementioned model, single shaft design, is used in this section. Microturbine, the PM generator, and the power conditioning system are shown in Fig. 2.2.

\section{A Modeling of MT}

Turbine has different control systems including speed control, acceleration control, temperature control, fuel flow control. These control systems are introduced as follows:

\section{1) speed control}

The speed control operates on the difference between a reference speed that is one per-unit and the synchronous generator rotor speed. In this part a lead-lag transfer function has been used for modeling the speed control. It is shown in Fig. 2.3. LVG is the low value gain which selects the least value of its inputs.

In this figure, $e$ is the controller gain, $a$ is the governor lead time constant, $c$ is the governor lag time constant and $d$ is a constant representing the governor mode. 


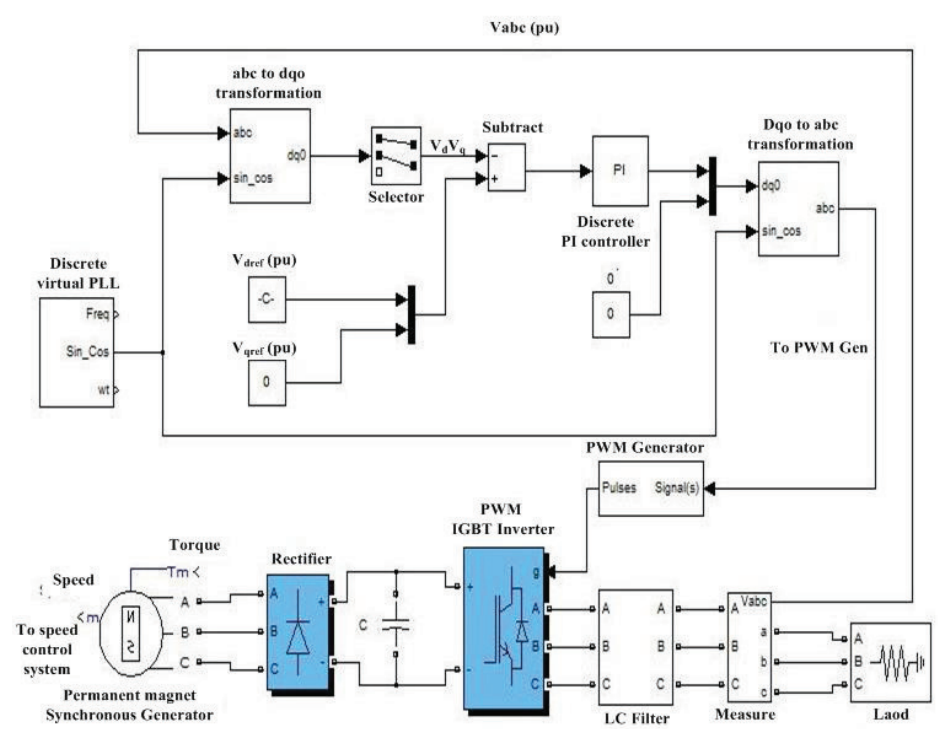

Fig. 2.7. permanent magnet generator, power conditioning system and voltage control scheme in simulink

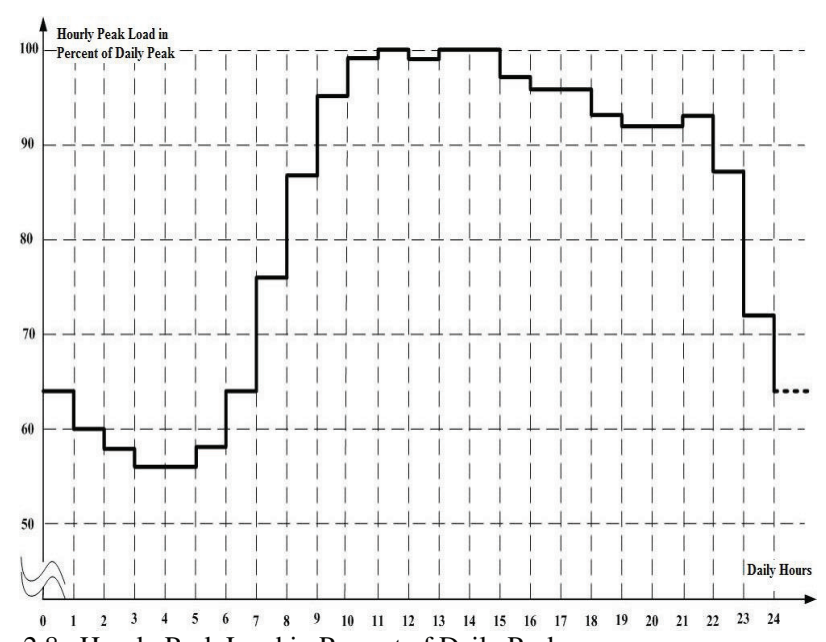

Fig. 2.8. Hourly Peak Load in Percent of Daily Peak

\section{2) acceleration control}

Acceleration control duty is to limit the rate of the rotor acceleration during turbine start up and reaching operating speed. This control system could be eliminated if the operation speed of the system is near its rated speed. This control system is presented in Fig. 2.4.

\section{3) Temperature control}

This control system limits the gas turbine output at a certain firing temperature with no regard to ambient temperature or fuel specification. The thermocouple measures the temperature of the exhaust gas of the turbine resulted from burning fuel flow in the combustor, and then compares it with a reference temperature. If the measured temperature exceeds the reference, the difference will become negative and it will start lowering the temperature control output. In the low value select part, when the temperature control output becomes the lowest compare to the governor output and acceleration control, the value will pass through and temperature control will run to limit the output. As a result, it reduces the fuel quantity to the combustor so reduces the exhaust temperature. When one of the speed governor output or acceleration control output becomes the least in the low level selection part, its output passes through and the unit gain operate on it. The temperature control system is presented in Fig. 2.5.

4) Fuel flow system

The fuel flow from the fuel system results from the inertia of the fuel system actuator and of the valve positioner. The equations are given below. The value positioner transfer function is:

$$
E_{f}=\frac{k_{v}}{T_{v} s+c} F_{d}
$$

where $k_{v}$ is the valve positioner gain, $T_{v}$ is the valve positioner time constant, $c$ is a constant and $F_{d}, E_{f}$ are input and output of the valve positioner.

And the fuel system actuator transfer function is:

$$
W_{f}=\frac{k_{f}}{T_{f} s+c} E_{f}
$$

where $K_{f}$ is the fuel system actuator gain, $T_{f}$ is the fuel system actuator time constant and $W_{f}$ is the fuel demand signal in p.u. 
KESHTKAR: MODELING AND CONTROLLING A HYBRID MULTI-AGENT BASED MICROGRID IN PRESENCE OF DIFFERENT PHYSICAL AND CYBER COMPONENTS

The output of the least value select part, $V_{c e}$, assigns the least amount of fuel needed for the operating point. It is one of the inputs to the fuel system [73]. Another input to the fuel system is the turbine speed in per-unit. The per unit value of $V_{c e}$ corresponds to the mechanical power of the turbine in steady state in per-unit. The fuel flow system is shown in Fig. 2.6.

The value of $V_{c e}$ is scaled by the gain. Then delayed and offset by the minimum amount of fuel flow at no-load to ensure continuous combustion process in the combustion chamber.

\section{B Permanent Magnet Synchronous Generator (PMSG) model}

A permanent magnet synchronous machine (PMSM) uses a permanent magnet for its field. Its advantages are elimination of field copper loss, higher power density, lower rotor inertia, more robust construction of the rotor, and higher efficiency. The disadvantages are loss of flexibility of field flux control, possible demagnetization, and generally higher cost [59].

The PMSM is analyzed in dq0 axis theory. For a balanced system, the 0 -axis is eliminated. The $\mathrm{dq}$ axis equations are formulated as follows:

$\frac{d i_{q s}}{d t}=\frac{1}{L_{q s}}\left[V_{q s}-R_{s} i_{q s}-L_{d s} \omega_{e} i_{d s}-\hat{\psi}_{f} \omega_{e}\right]$

$\frac{d i_{d s}}{d t}=\frac{1}{L_{d s}}\left[V_{d s}-R_{s} i_{d s}+L_{q s} \omega_{e} i_{q s}\right]$

$T_{e}=\frac{3 P}{4}\left[\hat{\psi}_{f} i_{q s}+\left(L_{d s}-L_{q s}\right) i_{q s} i_{d s}\right]$

$\frac{d \omega_{r}}{d t}=\frac{1}{J}\left(T_{e}-T_{\text {shaft }}\right)$

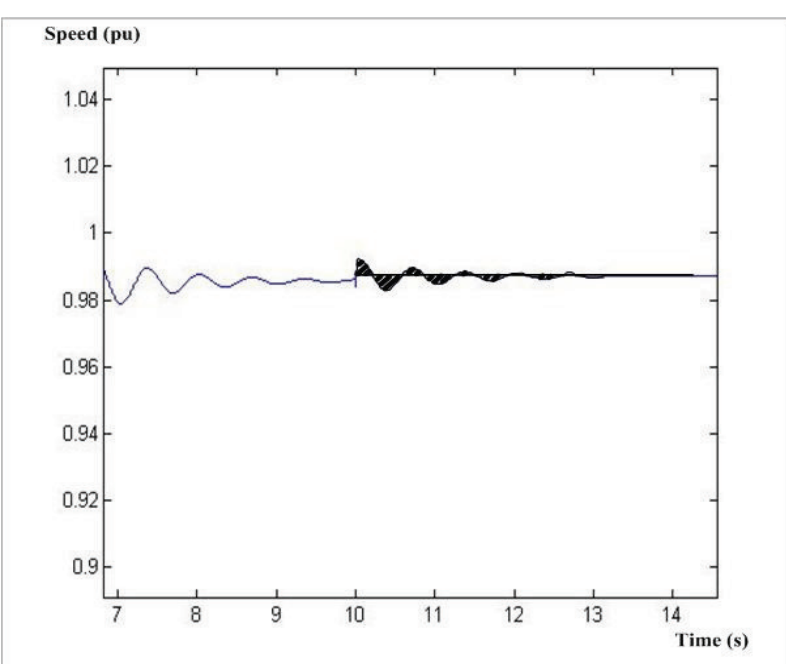

Fuel (pu)

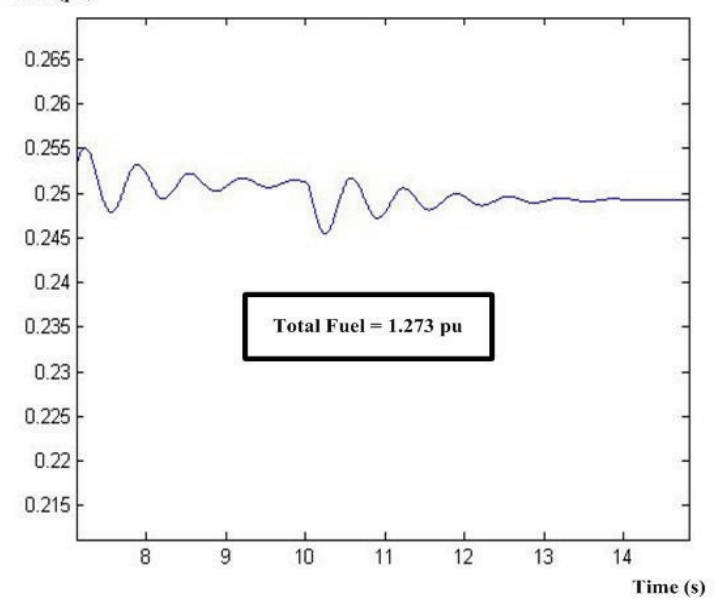

Fig. 2.9. (a) speed and (b) total fuel consumption in poor-controlled MTG system.

where $\omega_{e}, \omega_{r}$ are the electrical and mechanical angular velocities of the rotor in $\mathrm{rad} / \mathrm{sec}, V_{q s}, V_{d s}$ are the $\mathrm{q}$ and $\mathrm{d}$ axis voltage components, $I_{q s}, I_{d s}$ are the $\mathrm{q}$ and d axis current component, $L_{q s}, L_{d s}$ are the $\mathrm{q}$ and d axis inductances of the stator, $\psi_{f}$ is the flux linkage induced by the permanent magnets of the rotor in stator phases, $J$ is the inertia of the rotor $\left(\operatorname{Kgm}^{2}\right), T_{\text {shaft }}$ is the shaft torque produced by the microturbine $(\mathrm{Nm}), \mathrm{T}_{\mathrm{e}}$ is the electric torque generated by the PMSG $(\mathrm{Nm})$, and $P$ is the number of poles.

It is worth to mention that $T_{e}$ and $T_{\text {shaft }}$ are negative for generator operation and positive for motor operation.

\section{II.C Power conditioning and voltage control system}

Power electronics (rectifier-inverter) interface is used to control the frequency of output power of the MTG system. It controls the inverter output voltage quantity and angle, and maintains the frequency at a prescribed level. This is done by converting the high frequency AC power output from the generator to DC and then to $50 \mathrm{~Hz} \mathrm{AC}$.

Power conditioning unit also consists of a voltage source inverter and LC filter to reduce harmonic distortion and make the power quality criteria in better condition. 
A PI voltage regulator using abc to $\mathrm{dq}$ and $\mathrm{dq}$ to abc transformations is employed to regulate the load voltage at 480 volts. The output of the voltage regulator is used by the PWM generator to generate the gate pulses.

The permanent magnet generator, power conditioning system and voltage control scheme in simulink are shown in Fig. 2.7.

\subsection{Problem formulation}

The two most important output parameters of a microturbine system are fuel and speed. The importance factors that are considered about these two outputs are different. It is more applicable to consider total consumption of fuel as a quality criterion of fuel usage in microturbine system. The objective is to reduce the quantity of fuel using in a MTG system.

The quality of speed is related to its transient state. It is important to have suitable transient respond of speed against load changing. The minimum possible overshot and time of oscillations are usually considered to evaluate the speed transient respond. Since the speed specification is one of the factors that influence the transient quality of the fuel, by optimizing the speed governor system, the fuel suitable transient specification is achieved.

Furthermore, the issue of the stability of the MTG system is also considered in this objective. It will appear in this sort that if the system goes through the instability condition, the quality of this objective will reduce intensively and the results will be automatically evaluated as undesirable.

In this part, differential evolution (DE) algorithm has been proposed to optimize the control system of the speed governor. The control system has been shown in Fig. 2.10.

This method should be used in a practical case. Thus hourly peak load in percent of daily peek for a typical and standard summer week is considered as the load changes during the day and is presented in Fig. 2.8 [60].

The objective is to achieve the least amount of fuel consumption and the best transient quality of speed, when one steady state is switched to another. The new steady state is a function of the load variations. So the outputs in transient time between the steady states are considered to study. As there is the ability in modern control devices to make changes in the parameters of the controllers during the operation, it is possible to prepare a look-up table and implement the proposed control method for 24-hour load changes.

The load change at $12 \mathrm{am}$ is considered. With assuming the nominal load as $\mathrm{P}_{0}$, load would change from $0.72 \mathrm{P}_{0}$ to $0.64 \mathrm{P}_{0}$ based on Fig. 2.8. The speed and the fuel output would have low qualities without suitable controller. In other words, as shown in Fig. 2.9, the speed output has unacceptable transient respond to load change and oscillates a lot, and fuel consumption is high when the speed governor system is not adjusted appropriately.

In the proposed method, at first weighting factor should be defined for the considered objectives. The first objective is total fuel consumption and the second is speed transient response after load change. The better condition in one objective results worse in other. So a trade-off should be implemented between them.

The fitness testing criteria for the objectives are the total fuel consumption after load changing, and the total speed deviation of the final speed quantity in steady state after load changing.

So the total fuel consumption after load changing is defined as follows:

$$
x_{1}=\int_{t_{0}}^{t_{0}+T} f(t) \cdot d t
$$

and the total speed deviation of the speed after load changing is defined as follows: 
KESHTKAR: MODELING AND CONTROLLING A HYBRID MULTI-AGENT BASED MICROGRID IN PRESENCE OF DIFFERENT PHYSICAL AND CYBER COMPONENTS

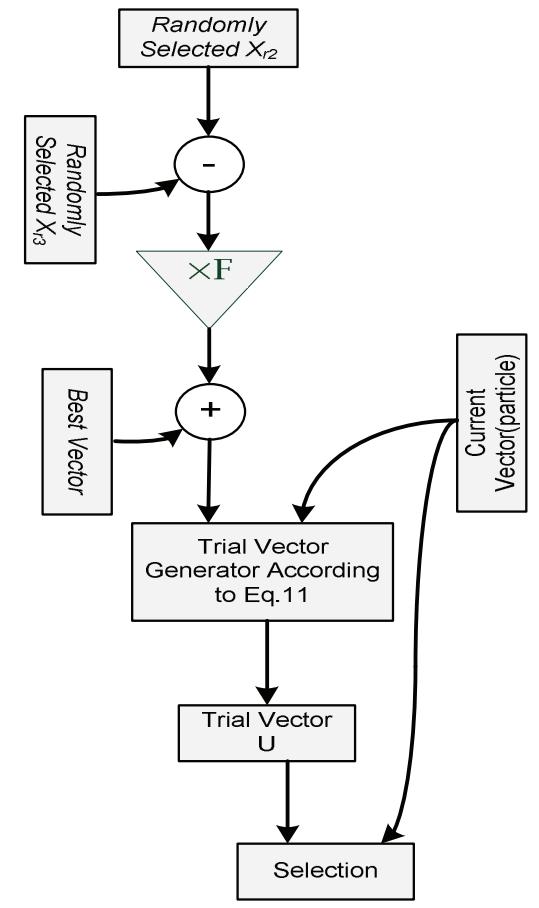

Fig. 2.10. Main idea of DE improvement attempts.

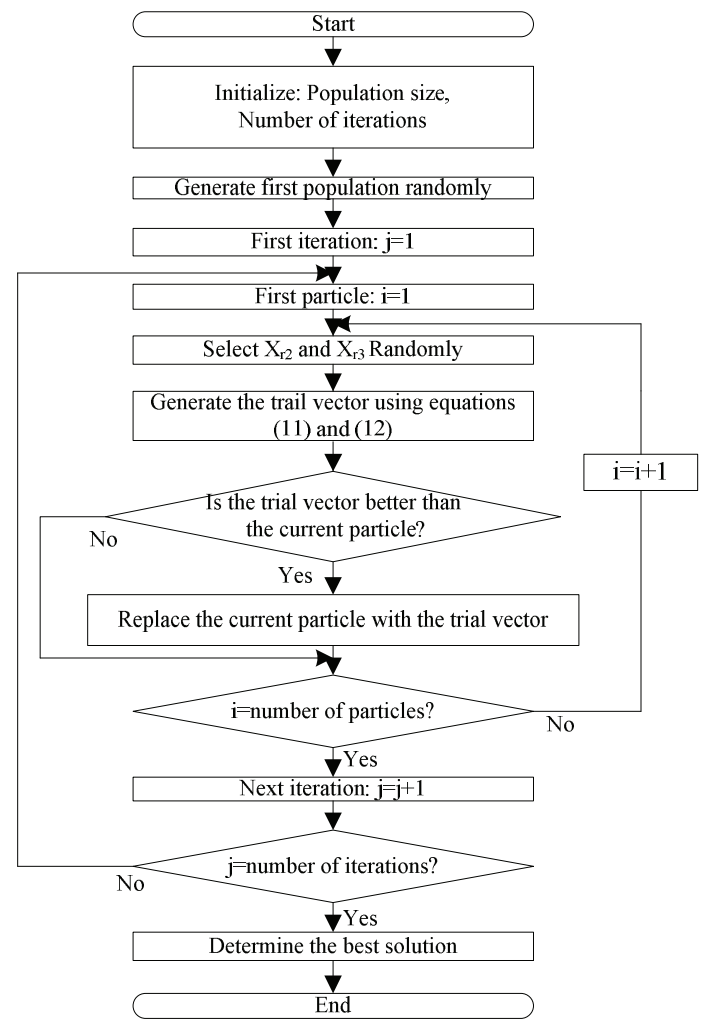

Fig. 2.11. Flowchart of DE.

$$
x_{2}=\int_{t_{0}}^{t_{0}+T}\left|\omega(t)-\omega_{T}\right| \cdot d t
$$

where $t_{0}$ is the time of load changing, $T$ is the steady state time after load changing, $x_{1}$ is the criteria quantity of first objective, $x_{2}$ is the criteria quantity of second objective, $f(t)$ is the fuel consumption function, $\omega(t)$ is the speed function and $\omega_{T}$ is the final speed in steady state. The later objective is defined on the concept of minimizing the colored area in Fig. 2.9(a).

The fitness testing criteria for the two objectives is given in Eq.(2.14),(2.15). For an example in Fig. 2.9, $t_{0}$ is 10 s and $T$ is 5 seconds after $t_{0}$. Thus, the criteria can be calculated. Note that Fig. 2.9(b) shows the amount of fuel consumed at the moment and the value in the box is the total consumption in the specified period of time.

\subsection{Multi-objective optimization handling}

\section{A. Implementation of Differential Evolutionary in Speed Governor Design Problem}

In this part, Differential Evolutionary (DE) Algorithm and its implementation for speed governor control problem is presented [73]. DE is a simple population based, stochastic parallel search evolutionary algorithm for global optimization and is capable of handling non-differentiable, nonlinear and multi-modal objective functions [61] that attacks the starting point problem by evaluating the objective function at multiple random initial points. A population composed of NP individuals evolves over several generations to reach an optimal solution. The main idea of the improved DE optimization is illustrated in Fig. 2.10. It includes several steps that are explained in this section. 


\section{1) Initialization}

The first generation consists of a population of NP vector with D dimension, while D is the number of control variables. In the proposed approach the population individual's vector will contain the parameters in speed governor system:

$$
x_{i}=[a, c, d, e]
$$

where $a, c, d$ are the parameters of the speed control system and $e$ is the gain of speed governor system.

In the initialization process, a lower bound $x_{j, \text { min }}$ for each variable as well as an upper bound $x_{j, \text { max }}$ are determined as will be described in part B. Once initialization bounds have been specified, a random number generator assigns parameter of every vector a value within the pre-specified range. For example, the initial value of the $\mathrm{j}^{\text {th }}$ variable of the $\mathrm{i}^{\text {th }}$ vector is:

$$
x_{i, j}=\operatorname{rand}(0,1) \cdot\left(x_{j, \max }-x_{j, \min }\right)+x_{j, \min }
$$

\section{2) Fitness Evaluation}

The goal of optimization is to minimize the objective function as discussed in section 2.2. However this optimization is along with satisfaction of several constraints. Since the constraints of this problem are implied on particle variables, one usual way to meet the constraints is to consider them during the process. This is described in part 6.

\section{3) Determining the Global Best Individual}

In each iteration of DE algorithm, a comparison between individuals is made to determine the best particle $x_{\text {best }}$. To specify the $x_{\text {best }}$, all the members of the current generation need to compete with each other. For the first generation, $x_{\text {best }}$ is the best one in the initial population [73]. For the $\mathrm{k}_{\mathrm{th}}$ generation, $x_{\text {best }, k}$ is obtained and compared with the last global best individual $x_{b e s t}$. If the $x_{b e s, k}$ is better than $x_{\text {best }}$, it takes $x_{\text {best }}$ place; otherwise $x_{\text {best }}$ remains unchanged.

\section{4) Mutation}

In DE, a mutant vector is produced from each individual. This step can be described as follows:

$$
\mathrm{V}_{i, K+1}=X_{r 1, K}+F \cdot\left(X_{r 2, K}-X_{r 3, K}\right)
$$

where $X_{r 2, K}$ and $X_{r 3, K}$ are randomly chosen vectors (particles) among the population in the generation $\mathrm{K}, F$ is a constant within $(0$, 2), and $V_{i, K+l}$ is the trial vector. If $X_{r l, K}$ is replaced by $X_{b e s t, K}$, another form of DE called BDE will be formed. Here, BDE is employed due to better results than conventional DE and $\mathrm{F}$ is set to 0.5 .

\section{5) Cross Over}

In the next step called the crossover step, the following equation is used to determine the trial vector that may replace the current vector in the next population with the probability of crossover constant (CR) which is between 0 and 1 [62]:

$$
U_{i, j, k+1}= \begin{cases}V_{i, j, k+1} & \text { if rand }(0,1) \leq C R \text { or } j=i \\ X_{i, j, k+1} & \text { otherwise }\end{cases}
$$

where rand $(0,1)$ is a random number generated within the range 0 and 1 and $C R$ is the crossover rate and set to 0.5.

\section{6) Handling the Constraints}

To ensure that all the variables of trial vector are within their limits, the following procedure is performed:

$$
\begin{array}{ll}
\text { If } U_{i, j, k+1}>x_{j, \text { max }} & \text { then } U_{i, j, k+1}=x_{j, \text { max }} \\
\text { If } U_{i, j, k+1}<x_{j, \text { min }} & \text { then } U_{i, j, k+1}=x_{j, \text { min }}
\end{array}
$$




\section{KESHTKAR: MODELING AND CONTROLLING A HYBRID MULTI-AGENT BASED MICROGRID IN PRESENCE}

(

Speed (pu)

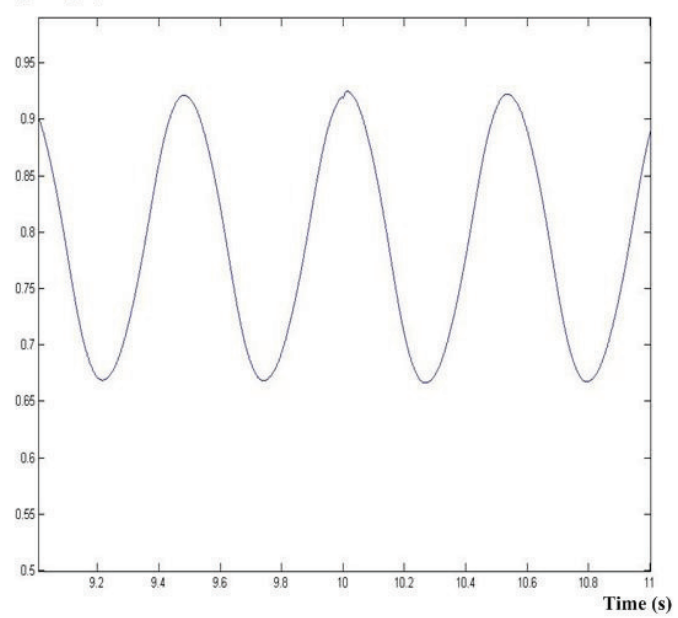

Fuel (pu)

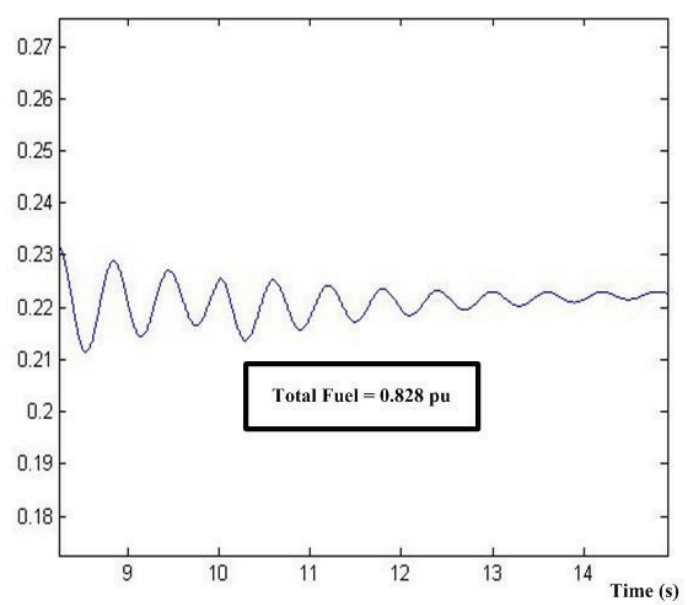

Fig. 2.12. (a) speed and (b) total fuel consumption for total fuel weighted optimal control design.
[S
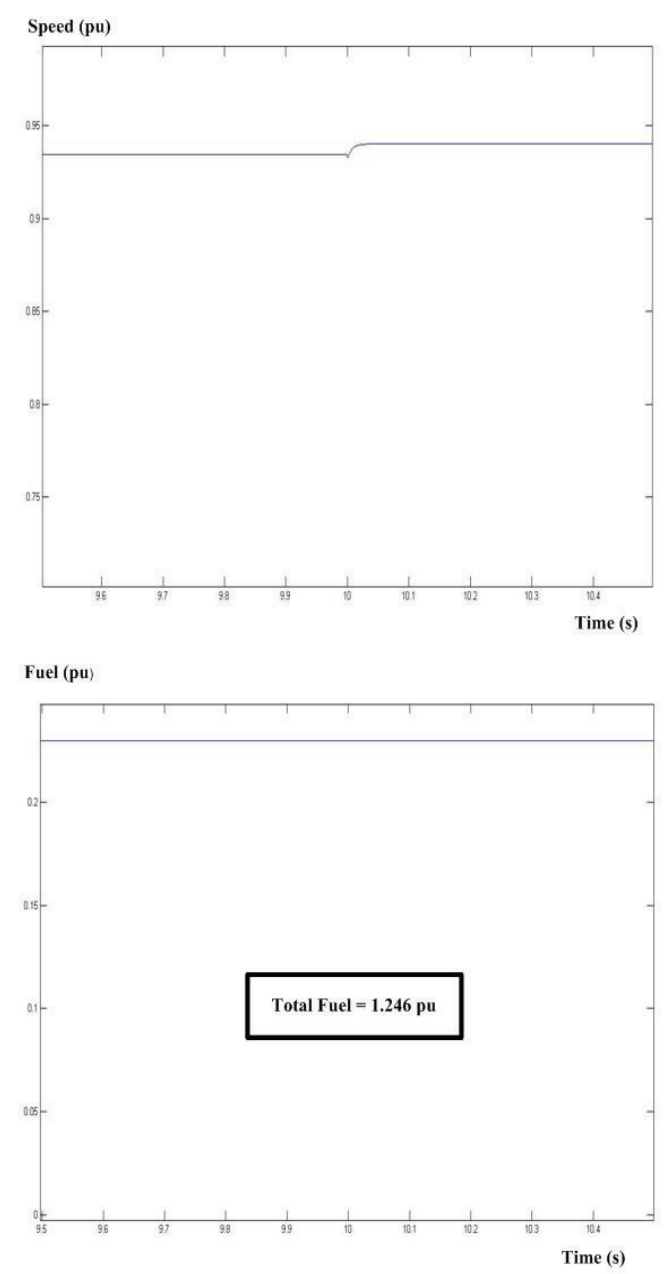

Fig. 2.13. (a) speed and (b) total fuel consumption for speed weighted optimal control design.

\section{7) Selection}

Finally, like other evolutionary algorithms, the selection phase is performed and the generated vector is tested comparing the best vector of prior iteration. If the trial vector resulted in a better fitness function, than the current particle, it will take the current particle's place. If not, the current one stays.

In $\mathrm{BDE}$, these steps are repeated in times of a defined number of iterations and the algorithm is terminated if the stop circumstances are confirmed. Fig. 2.11 shows the flowchart of DE algorithm.

\section{B. Implementation of Normalizing Technique in Speed Governor Design Problem}

The four steps which are taken to handle the multi-objective problem are described in this part. The expression which is considered to be optimized is:

$$
f=\sum_{i=1}^{n} c_{i} \cdot \frac{x_{i}-x_{i_{\min }}}{x_{i_{\max }}-x_{i_{\min }}}
$$



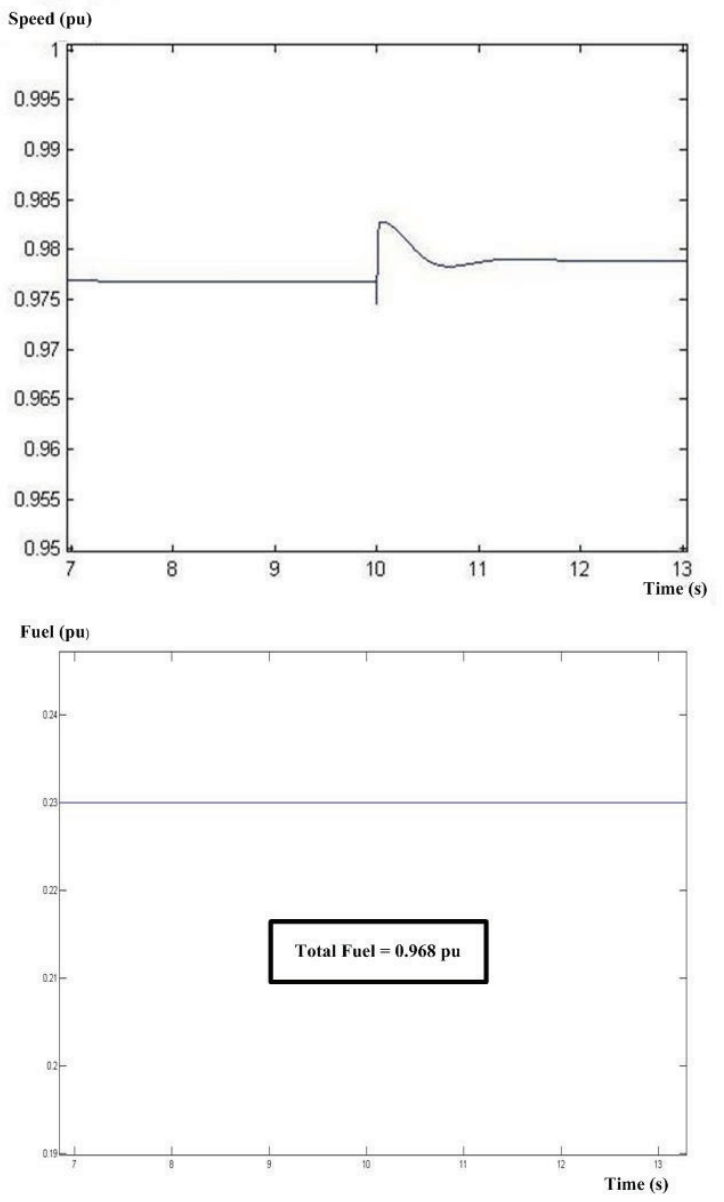

Fig. 2.14. (a) speed and (b) total fuel consumption for the suitableweighted speed and total fuel optimal control design. where $n=2$ is the number of objectives, $c_{i}$ is the weighting factor of objective $\mathrm{i}, x_{i}$ is the quantity of objective $\mathrm{i}, x_{i_{\min }}, x_{i_{\max }}$ are the minimum and maximum quantity of objective $i$. The four steps to find optimized parameters of the speed governor system $(a, c, d, e)$ are mentioned below.

Step1:

At first, the weighting factor for total fuel consumption is considered to be equal to 1 versus 0 for speed. So the optimization is run for the first objective, which is minimizing fuel consumption as shown in Eq. (2.14), and it will result the best condition for this objective.

Step2:

The weighting factor for speed is considered to be equal to 1 versus 0 for total fuel. So the optimization is applied for another objective, which is minimizing speed deviation as sown in Eq. (2.15), and the best condition for this objective will be obtained.

Step3:

In this step, the maximum quantities for both objectives are obtained.

Step4:

In this step, the weighting factors for both objectives are considered to be equal to the best quantity related to the best total responds. Thus the

best control design of speed governor for both objectives will be obtained.

In BDE, these steps are repeated in times of a defined number of iterations and the algorithm is terminated if the stop circumstances are confirmed. Fig. 2.11 shows the flowchart of DE algorithm.

At first, step1 should be implemented. So the optimization will result the best condition for total fuel. As shown in Fig. 2.12, the total fuel consumption at its least possible quantity. Secondly, step2 is done and the optimization will result the best condition for speed. As shown in Fig. 2.13, the speed output is suitable and almost without variations. It is resulted that these two objectives are in contrast with each other. In step3, suitable importance is allocated for both total fuel and speed. Finally, the best control design for both objectives will be obtained in step4. As shown in Fig. 2.14, the both objectives of total fuel and speed deviation are in their best possible condition, simultaneously.

\section{PHEV PENETRATION IN MICROGRID}

\subsection{Problem Formulation and System Modeling}

In this part, configuration of the model system in the presence of PHEVs is introduced and the control problem for frequency control in this system is described. Then, different stochastic behavior of PHEV is modeled in the system. In this part, integral control and intelligent control methods have been used to optimize the parameters of speed governor lead-lag controller in conventional LFC methods. 


\section{KESHTKAR: MODELING AND CONTROLLING A HYBRID MULTI-AGENT BASED MICROGRID IN PRESENCE OF DIFFERENT PHYSICAL AND CYBER COMPONENTS \\ A. System Configuration and Simulation Model}

System considered in this part is the hybrid power generation which introduced previously. Power supplied to the load $\mathrm{P}_{\mathrm{s}}$ is the sum of output power from wind turbine generators $\mathrm{P}_{\mathrm{w}}$, diesel generators $\mathrm{P}_{\mathrm{g}}$, photovoltaic generation $\mathrm{P}_{\mathrm{pv}}$, total loss power $\mathrm{P}_{\mathrm{Loss}}$ and output of PHEV $\mathrm{P}_{\text {phev }}$ given by

$$
P_{s}=P_{w}+P_{g}+P_{p v}+P_{l o s s} \pm P_{p h e v}
$$

LFC system in this part uses this power flow balance equation for adjusting the frequency of the microgrid. Modeling of different parts of the system is discussed in different sections.

\section{1) Modeling of Wind Turbine Generator}

The wind turbine is characterized by non-dimensional curves of power coefficient $\mathrm{Cp}$ as a function of both tip speed ratio $\lambda$ and blade pitch angle $\beta$. The tip speed ratio, which is defined as the ratio of the speed at the blade tip to the wind speed, can be expressed by

$$
\lambda=\frac{R_{\text {blade }} \omega_{\text {blade }}}{V_{W}}
$$

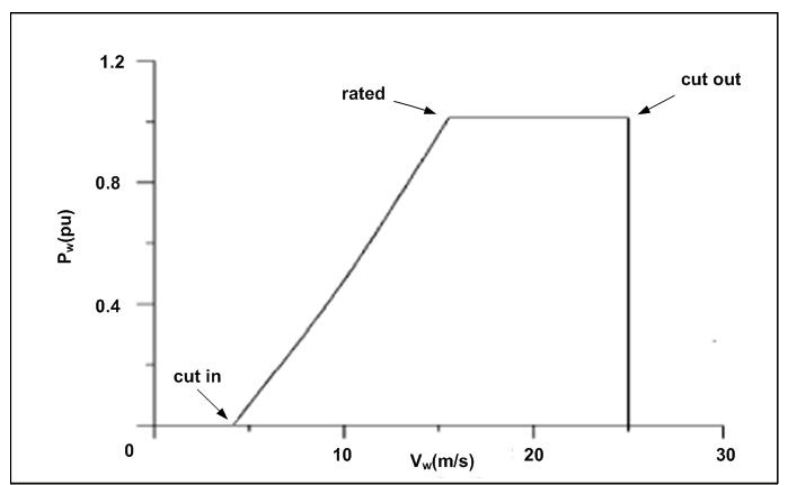

Fig. 2.15.Characteristic curve of output mechanical power versus wind speed of the studied WTGs.

Where $\mathrm{R}_{\text {blade }}(=23.5 \mathrm{~m})$ is the radius of blades and $\omega_{\text {blade }}(=3.14 \mathrm{rad} / \mathrm{s})$ is the rotational speed of blades. The expression for approximating $C_{p}$ as a function of $\lambda$ and $\beta$ is given by

$$
C_{p}=(0.44-0.0167 \beta) \sin \left[\frac{\pi(\lambda-3)}{15-0.3 \beta}\right]-0.0184(\lambda-3) \beta
$$

The output mechanical power of the studied WTGs is

$$
P_{W}=\frac{1}{2} \rho A_{r} C_{p} V_{W}^{3}
$$

where $\rho\left(=1.25 \mathrm{~kg} / \mathrm{m}^{3}\right)$ is the air density and $\mathrm{A}_{\mathrm{r}}\left(=1735 \mathrm{~m}^{2}\right)$ is the swept area of blades. The characteristic curve of output mechanical power versus wind speed of the studied WTGs in this section is shown in Fig. 2.15.

\section{Distribution}

Power system

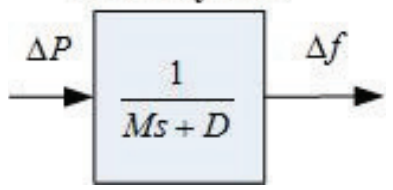

Fig. 2.16. Model of distribution power system 


\section{Device i}

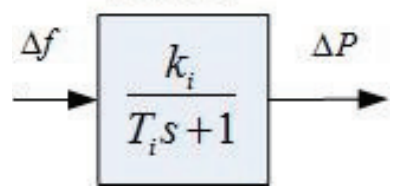

Fig. 2.17. Model of generation subsystem devices

\section{2) Modeling of Distributed Power System and Various Generation Subsystems}

The distributed power system and different generation subsystems are modeled as shown in Fig. 2.16 and Fig. 2.17, respectively. The parameters of the distribution power system and different generating units such as Photovoltaic (PV), Wind Turbine Generator (WTG), Diesel Engine Generator (DEG), are presented in Table 2.II.

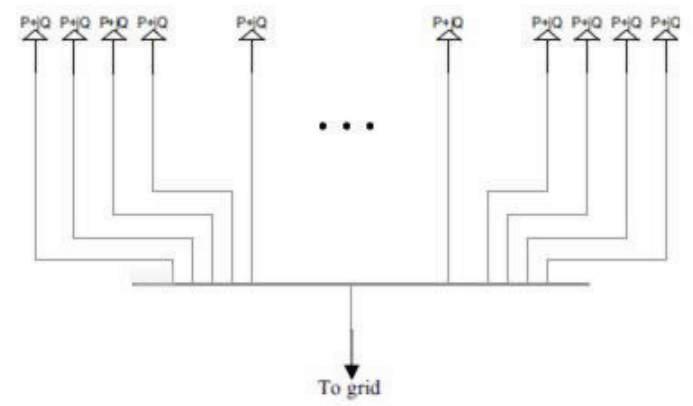

Fig. 2.18. PHEVs connection to the microgrid

\section{3) Modeling of PHEV}

The PHEV in this work is modeled as a load. Fig. 2.18 shows how PHEVs are connected to the grid. In this case, effect of different PHEV penetration into the microgrid can be studied. The number of PHEVs in the system can depend on the amount of load that we have. But the maximum number is not more than $18 \%$ of total loads. Other than PHEVs function as load it can have a function as energy storage of the system in case of disturbances in the frequency output of the system. Fig. 2.19 shows PHEVs model comprise of mechanical and electrical parts. It includes power conditioning system to convert DC power of batteries and single phase power to three phase power for generator and motor systems. The mechanical system does not get involved in charging/discharging the battery for frequency control problem.

Table 2.II. Parameters of the studied system

\begin{tabular}{|l|l|l|l|l|l|l|l|}
\hline $\begin{array}{l}\text { Distribution } \\
\text { Power system }\end{array}$ & \multicolumn{2}{|l|}{ PV } & \multicolumn{2}{l|}{ WTG } & \multicolumn{2}{l|}{ DEG } \\
\hline$M$ & $D$ & Kpv & Tpv & Kwtg & Twtg & Kdeg & Tdeg \\
\hline 10 & 1 & 1 & 1.8 & 1 & 1.5 & 1 & 2 \\
\hline
\end{tabular}

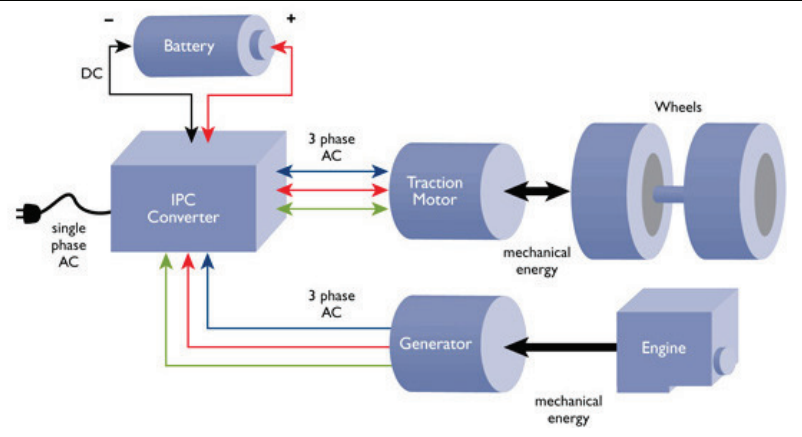

Fig. 2.19. PHEV Model 
KESHTKAR: MODELING AND CONTROLLING A HYBRID MULTI-AGENT BASED MICROGRID IN PRESENCE

OF DIFFERENT PHYSICAL AND CYBER COMPONENTS

3.2 Overview of Particle Swarm Optimization (PSO)

The control parameters of the generator systems and PHEV in the studied power microgrid system are optimized by Particle Swarm Optimization (PSO) Algorithm. Therefore, this algorithm is discussed in this part. This algorithm was also described in part 2.3 of chapter one for optimal PV placement. However, its application for the problem discussed in this section is needed.

PSO is a multi-agent search technique, which traces its evolution to the emergent motion of a flock of birds searching for food [73]. It uses a number of particles that constitute a swarm. Each particle traverses the search space looking for the global minimum (or maximum). In a PSO system, particles fly within a multidimensional search space. During flight, each particle adjusts its position according to its own experience as well as the experience of neighboring particles, making use of the best position encountered by itself and its neighbors. Similarly, the swarm direction of a particle is determined by the history experience obtained by itself a set of its neighboring particles.

Let us denote $p$ and $s$ as particle coordinates (position) and its corresponding flight speed (velocity) in a search space, respectively. The best previous position of a particle is recorded and represented as $P_{\text {best }}$. The index of the best particle among all the particles in the group is represented as $G_{b e s t}$. To ensure convergence of PSO, Eberhart indicates that use of a constriction function may be necessary. At last, the modified velocity and position of each particle can be calculated as shown in (2.26) and (2.27):

$$
\begin{aligned}
& s_{d+1}=k *\left(\gamma ^ { * } v _ { d } + a c _ { 1 } \text { rand } \left(0 *\left(P_{b e s t}-P_{d}\right)+a c_{2} * \operatorname{rand}\left(0 *\left(G_{\text {best }}-P_{d}\right)\right)\right.\right. \\
& P_{d+1}=P_{d}+s_{d+1}
\end{aligned}
$$

where $\mathrm{d}$ is the index of iteration, $P_{d}$ is the current position of particle at the d-th iteration, $s_{d}$ is the velocity of particle at d-th iteration, $\gamma$ is inertia weight factor, $a c_{1}$ and $a c_{2}$ are acceleration constants, rand() is a uniform random value in the range [0,1] [75], and $\mathrm{k}$ is the constriction factor which is a function of $a c_{1}$ and $a c_{2}$ according to (2.28):

$$
k=\frac{2}{\mid 2-a c-\sqrt{a c_{2}-4 a c \mid}}
$$

Where $a c=a c_{1}+a c_{2}$ and $a c>4$. Appropriate selection of inertia weight, $\gamma$, provides a balance between global and local explorations. In general, $\gamma$ is set according to (2.29):

$$
\gamma=\gamma_{\max }-\frac{\gamma_{\text {max }}-\gamma_{\text {min }}}{\text { iter }_{\text {max }}} \times \text { iter }
$$

Where iter $_{\max }$ the maximum number of iterations and iter is the number of the iterations up to current stage.

Inertia weight $(\gamma)$ can be selected as constant or reducing with time. In case of variable inertia weight, $\gamma$ is firstly selected in range of [0.8-1.2] to weight the global search and then it is decreased to favor the local search. Several studies propose different values for these parameters that are considered adequate for some of the usual benchmark functions in literatures [15].

By defining a limit for increasing the velocity of particles $\left(s^{\max }\right)$ the region in which the particles can search for obtaining the best fitness will be specified. By utilizing this limit, the effect of changes in learning procedure will be considered and local search

ability of the algorithm will be improved. $s^{\max }$ is usually selected based on the dynamic of optimization variables and can take different values. These values affect the ability of the algorithm in finding the optimal solution. For instance, assigning large $s$ max 
may lead the algorithm to lose the best solution, and selecting small $s^{\max }$ may result in finding local minimum instead of the global one. In literatures, $s^{\max }$ is usually set at $0.1-0.2$ of the dynamic range of the variable on each dimension [74].

This optimization is going to be used for minimizing the frequency deviation of the system by adjusting the controller parameters of the generating units and PHEVs.

\subsection{Case Study}

Fig. 2.20 shows the microgrid system considered in this part. It can be seen that number of PHEVs in the system can be defined. This hybrid system comprises of different renewable energy sources such as WTG and PV, DEG as a distributed generation unit, PHEVs and loads.

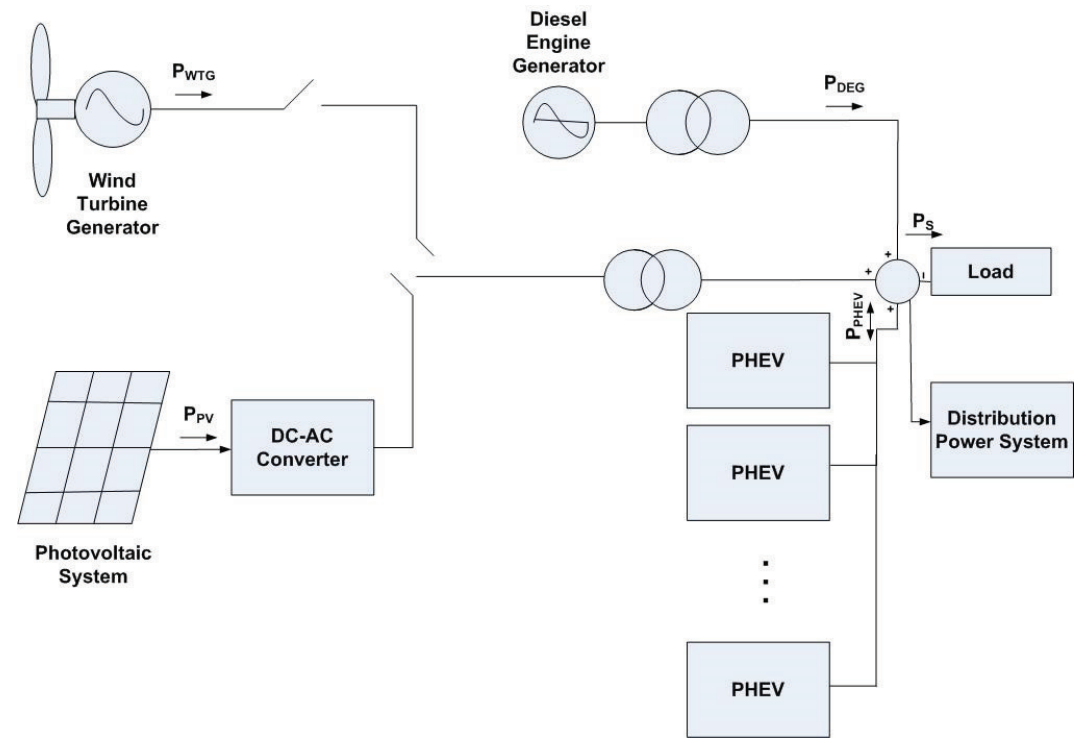

Fig. 2.20. Modeled microgrid with PHEV penetration

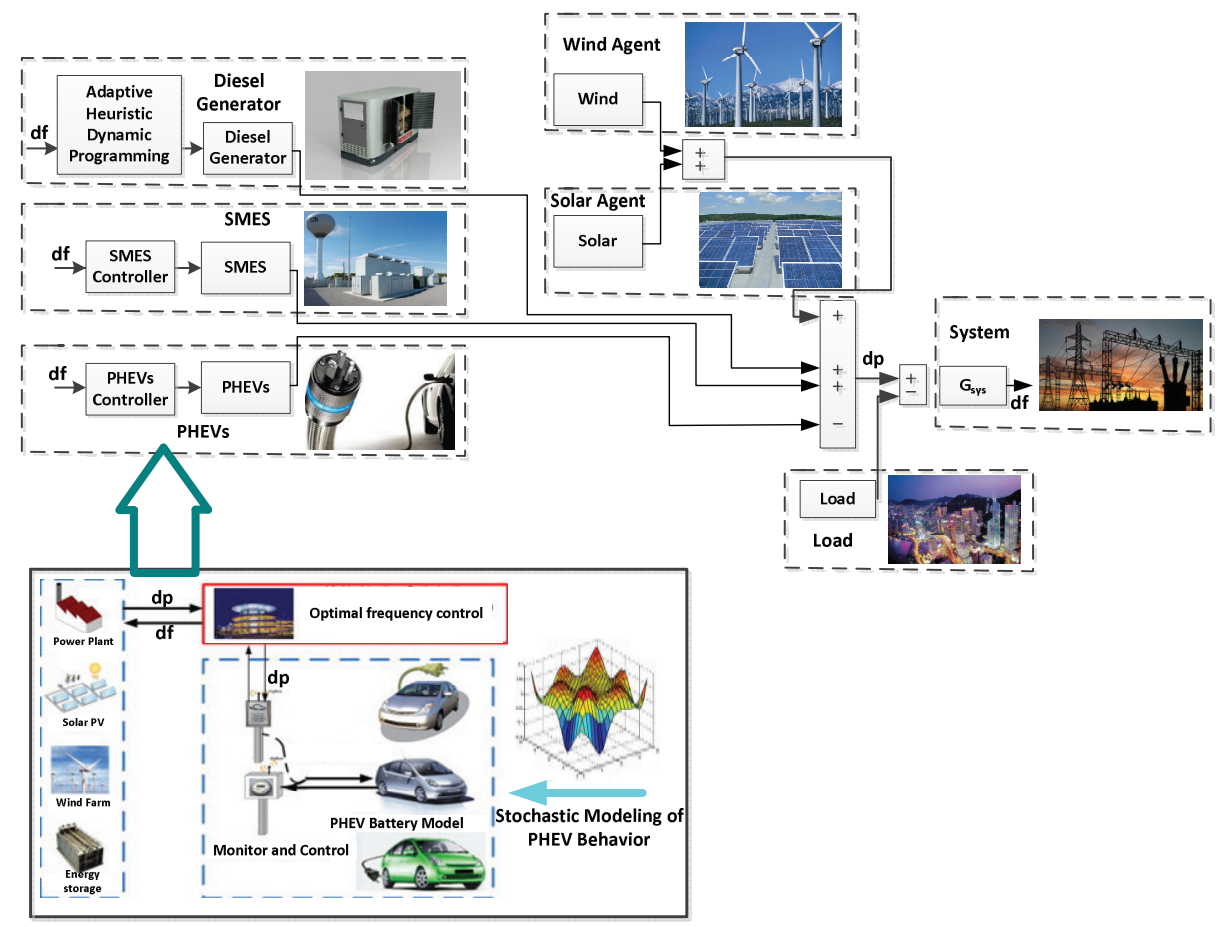

Fig. 2.21.Simulation model of the studied Microgrid 
KESHTKAR: MODELING AND CONTROLLING A HYBRID MULTI-AGENT BASED MICROGRID IN PRESENCE OF DIFFERENT PHYSICAL AND CYBER COMPONENTS

\subsection{Stochastic Behavior of PHEV}

In high penetration of PHEVs the stochastic behavior of these devices starts to affect the frequency control procedure. In this part these behaviors are discussed and modeled for PHEVs. The Simulation model of the modeled microgrid besides stochastic and controller modeling is shown in Fig. 2.21.

\section{A. Stochastic behavior Modeling}

Four different stochastic processes for modeling PHEV behavior are introduced and shown in this part.

\section{Brownian motion}

It is one of the second type random distributions which is very close to the PHEV pattern of charging and discharging in the microgrid. This is the continuous analog of symmetric random walk distributions, each increment $\mathrm{W}(\mathrm{s}+\mathrm{t})-\mathrm{W}(\mathrm{s})$ is $\mathrm{Gaussian}$ with distribution $\mathrm{N}(0, \mathrm{t})$ and increments over disjoint intervals are independent [48]. It is typically simulated as an approximating random walk in discrete time. In this study, charging and discharging of $300 \mathrm{PHEV}$ have been modeled by Brownian motion with sigma of 3 and it is shown in Fig. 2.23.a.

This disturbance is added to the wind and load deviation in the microgrid which are shown in Fig. 2.22.
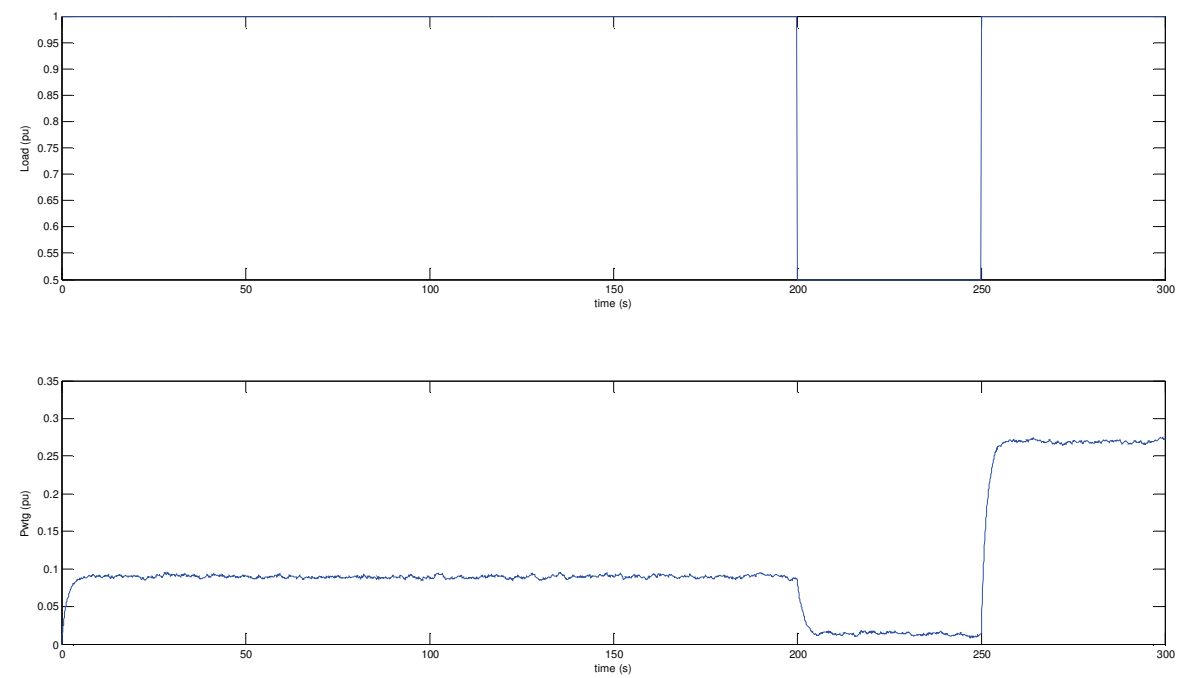

Fig. 2.22. Load and wind perturbation in the microgrid

Simulation result for frequency deviation before and after proposed control is shown in the simulation section.

\section{Poisson process}

It is another one of the second type random distributions which is very close to the PHEV pattern of arrival time for charging and discharging in the microgrid. It generate random events so that: (i) arrival occur independent of each other, (ii) two or more arrival cannot occur at the same time point, (iii) the arrival occur with constant intensity. Number of arrival N(t) that occur from the time zero up to time $\mathrm{t}$ is Poisson distributed with expected value lambda*t. The counting process $\mathrm{N}(\mathrm{t})$ is a Poisson process. The successive times between arrivals are Exponential (lambda) distribution. In this study, arrivals of 300 PHEV have been modeled by Poisson process with lambda of 3 and the total number of them in the microgridis shown in Fig. 2.23.b. The starting time of the arrival has been chosen by random walk distribution which is about $148 \mathrm{~s}$. 
This disturbance is added to the wind and load deviation in the microgrid which are shown in Fig. 2.22 and simulation results for frequency deviation before and after proposed control is shown in the simulation section.

\section{Brownian process $3 D$}

It is another one of the second type random distributions. In order to consider more than one type of the PHEV patterns of behavior higher dimensional random distributions is recommended. Brownian process 3D is appropriate to model the PHEV pattern of arrival time (number of PHEVs), time of charging/ discharging, and amount of charging/discharging in the microgrid. In this study, behavior of 300 PHEV have been modeled by 3D Brownian process with sigma of 2 and arrival time-time of chargingamount of charging of them in the microgrid is shown in Fig. 2.23.c.

The SOC is calculated based on Fig. 2.23.c and the resulted disturbance is added to the wind and load deviation in the microgrid which are shown in Fig. 2.22 and simulation results for frequency deviation before and after proposed control is shown in the simulation section.

\section{The $M / M / 1$ model}

It is one of the third type random distributions (Markov process) in the category of Queuing systems. This process is very close to the PHEV pattern of arrival time (number of PHEVs) for charging and discharging in the microgrid when PHEVs are charging / discharging at stations.

Suppose time is discrete and PHEV arrive to a service center according to an independent sequence a(1), a (2) ..., where $\mathrm{a}(\mathrm{k})$ is the number of arrivals during time slot number $\mathrm{k}$. One PHEV can be charged/discharged per slot (single server system). Additional PHEV wait in a line until service available. Then the number of PHEVs in the system at time $\mathrm{k}$ is given by $\mathrm{n}(\mathrm{k})=\mathrm{n}(\mathrm{k}-$ $1)+\mathrm{a}(\mathrm{k})-\mathrm{I}\{\mathrm{n}(\mathrm{k}-1)+\mathrm{a}(\mathrm{k})>=1\}, \mathrm{k}>=2$. With initial condition $\mathrm{n}(1)=0$. This recursion defines a Markov chain $\mathrm{n}(\mathrm{k}), \mathrm{k}>=1$.

$\mathrm{M} / \mathrm{M} / 1$ is a single server buffer model in continuous time. Arrivals to the system are determined by a Poisson process with intensity lambda. The server needs an exponentially distributed random time for each PHEV, mean service time $1 / \mathrm{mu}$. The resulting system size $\mathrm{N}(\mathrm{t}), \mathrm{t}>=0$, is a Markov process in continuous time which evolves as follows. Start from $\mathrm{N}(0)=\mathrm{n} \_0$ [52]. Wait an exponential time with intensity lambda+mu (intensity lambda if $\mathbf{n}_{-} 0=0$ ), then charge with probability lambda/(lambda+mu) and discharge with probability $\mathrm{mu} /(\mathrm{lambda}+\mathrm{mu})$.

In this study, behavior of 500 PHEVs has been modeled by M/M/1 model with lambda of 1.5 and mu of 0.8 . The total number of PHEVs in the microgrid is shown in Fig. 2.23.d.

The disturbance related to charging/discharging of these PHEVs is added to the wind and load deviation in the microgrid which are shown in Fig. 2.22 and simulation results for frequency deviation before and after proposed control is shown in the simulation section. 


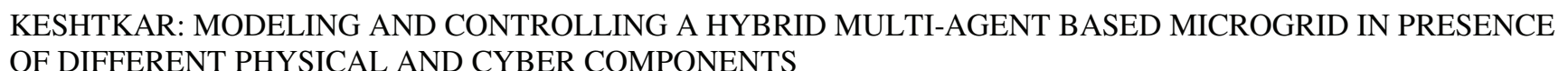
OF DIFFERENT PHYSICAL AND CYBER COMPONENTS
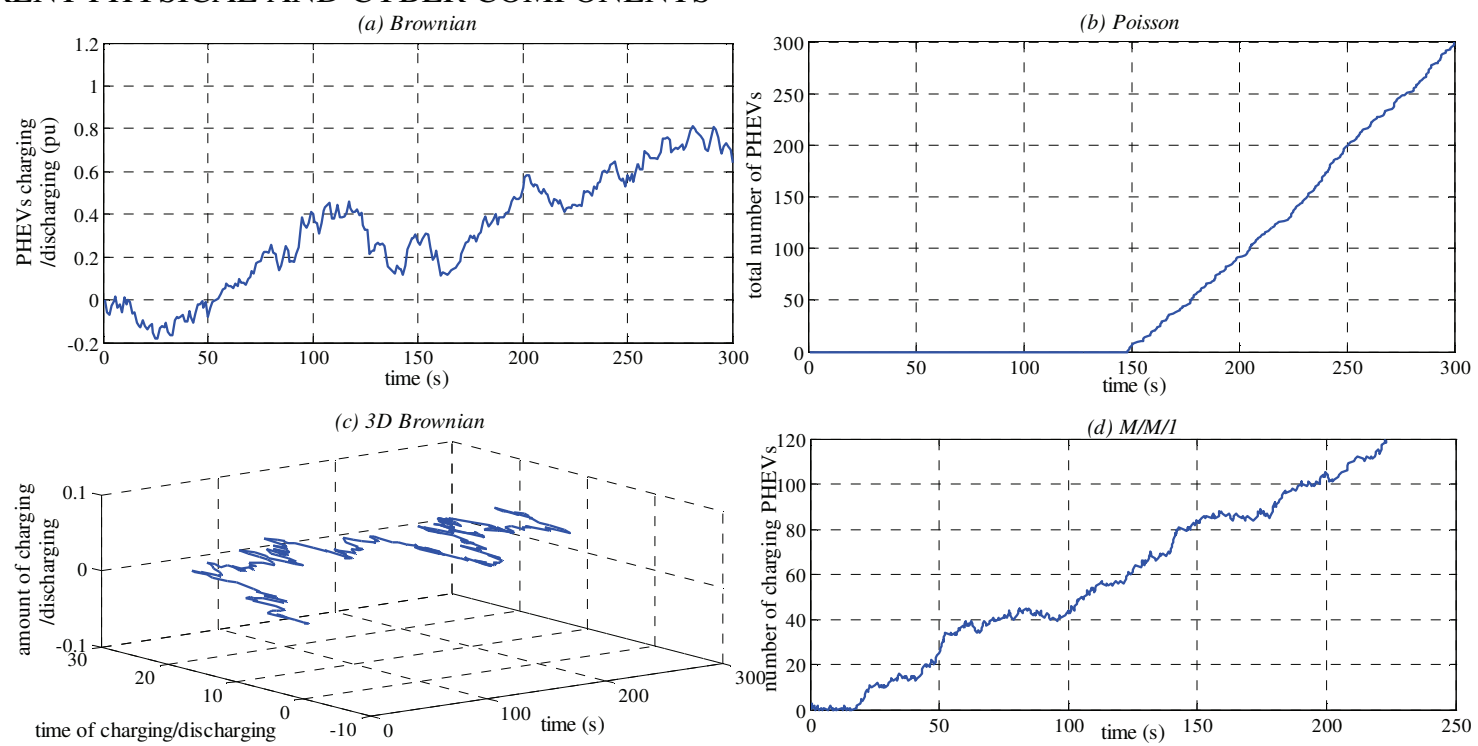

Fig. 2.23. Stochastic PHEV behavior modeling by; (a) brownian, (b) poission, (c) 3D Brownian, (d) M/M/1

\section{MULTI-AGENT BASED LFC FOR MICROGRID}

In this part, we have considered a 100\% self-sufficient isolated microgrid consisting of wind turbine generator (300 kW), solar power source $(300 \mathrm{~kW})$, diesel engine generator $(400 \mathrm{~kW})$, fuel cell $(200 \mathrm{~kW})$ and aqua-electrolyser $(100 \mathrm{~kW})$ for implementing a multi-agent system for frequency control. The total generation from renewable sources and from the controllable sources is equal to or more than total load which is $600 \mathrm{~kW}$. The scheme of the microgrid is shown in Fig. 2.24.

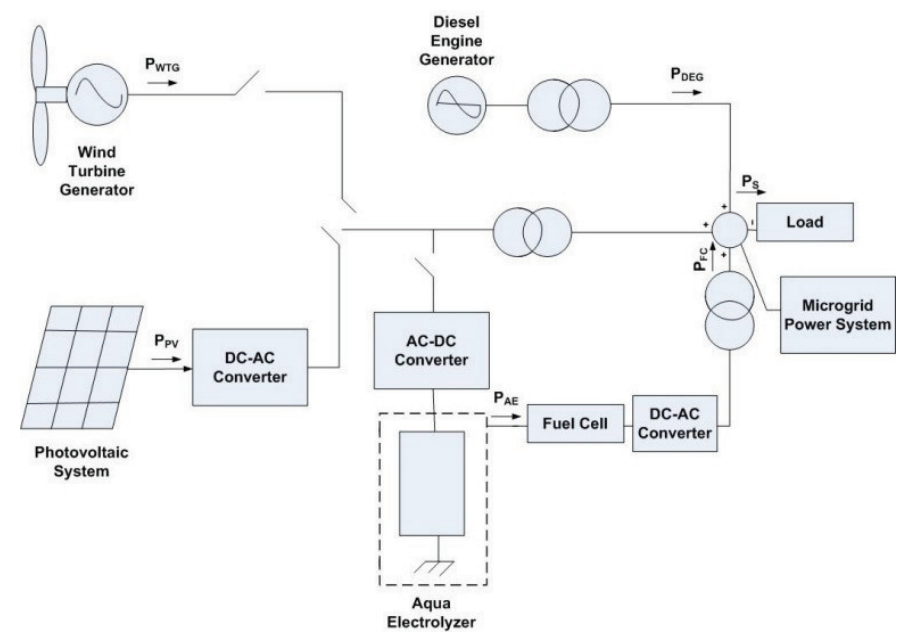

Fig. 2.24. The scheme of the modeled microgrid.

\subsection{Modeling of Microgrid and Problem Formulation}

\section{A. Multi Agent System}

Multi Agent Reinforcement Learning (MARL) is one of the adaptive and intelligent control techniques that have found little attentions in the LFC design. As it is based on learning, it can learn each kind of environment disturbances, and can be easily scalable for large scale systems [63-66]. Eventually, the multi-agent system (MAS) was proposed and recognized as one of the effective solutions for coordinating the distributed units in islanded microgrids [67-68]. 
The load-frequency control (LFC) problem has been one of the major subjects in a power system. In practice, LFC systems use proportional-integral (PI) controllers. However since these controllers are designed using a linear model, the non-linearities of the system are not accounted for and they are incapable of gaining good dynamical performance for a wide range of operating conditions in a microgrid power system. A strategy for solving this problem because of the distributed nature of a microgrid power system is to use a multi-agent reinforcement learning approach [13].

There are two main types of MASs: centralized and decentralized multi-agent system (CMAS and DMAS). If only CMAS structure is used, a central control agent is required to handle huge amounts of data, which is expensive and easily suffer cyber security risks. In addition, the CMAS, alone, lacks the adaptability to meet the plug-and-play operation requirements of microgrids. Furthermore taking uncertainty of intermittent renewable energy resources into consideration, generation fluctuation may result in unintentional structure changes and will further increase the burden to centralized schemes [68]. However, the DMAS along with CMAS can overcome the above mentioned shortcomings. Advantages of the DMAS including decentralized data processing and decision making will be added to the controller system, which leads to efficient task distribution, eventually causing faster operation and decision making process. A centralized multi-agent structure which is improved with adding some aspects of distributed one is used and will be explained in this section.

\section{A.1. Proposed approaches and algorithms}

A multi-agent based control architecture using reinforcement learning is proposed to enhance microgrid stability. It consists of a layer of local agents and a global agent that coordinates the behavior of the local agents.

The controllers are decentralized controllers whose objectives are to satisfy a local performance index or a local Lyapounov function. A coordinator and central controller communicates with the local controllers to satisfy a global performance index or a total energy function. Each local agent should also be able to handle the control parameters of its area in case of any contingency on communications toward the global agent.

\section{A.2. Configuration and Modeling}

Frequency control architecture for microgrid system is shown in Fig. 2.25. This multi-agent based configuration includes the power system model, measurements, the microgrid controller and the communication links. It illustrates how the frequency measurement signals are transmitted through communication links to the central controller agents in order to assign the proper frequency control actions for the actuators.

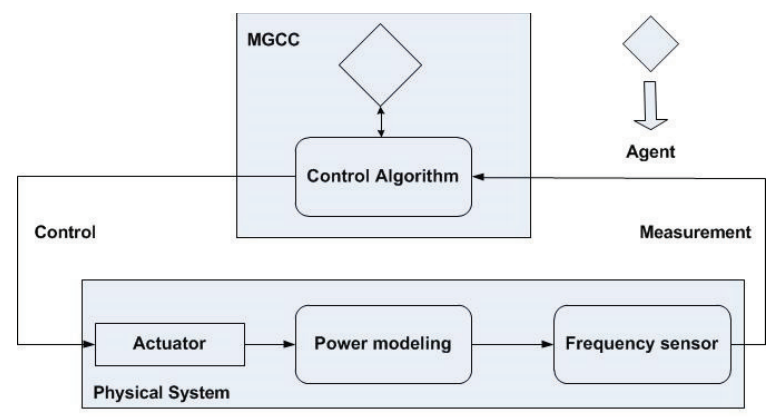

Fig. 2.25. Configuration of the proposed multi agent system for LFC in microgrid

The modeling of the multi-agent microgrid with more detail is shown in Fig. 2.26. It is shown that each generation unit and load node has been assigned with a local agent which is communicating with a global agent. The global agent contains a central controller unit which is described in the next part and is responsible for coordinating the local agents in order to optimize a global cost function. Each agent is also capable of analyzing the situation and making decision in case of contingencies and loss of 
KESHTKAR: MODELING AND CONTROLLING A HYBRID MULTI-AGENT BASED MICROGRID IN PRESENCE OF DIFFERENT PHYSICAL AND CYBER COMPONENTS

communication with the global agent. All local agents are also able to communicate with each other. This configuration in microgrid is called distributed multi-agent structure. The WTG and PV local agents in this figure are uncontrollable sources which are supplying the system based on the available renewable energy source. The rest of the source local agents are controllable sources which are mostly used for frequency control purposes.

\section{B. Microgrid Central Controller (MGCC)}

A central controller is designed in the global agent to manage the resources in the system based on the status of the loads and sources. Each load and source is considered as an agent and the status is obtained with the help of multi-agent concept communication over User Datagram Protocol/Internet Protocol (UDP/IP) as shown in Fig. 2.27. The decisions are sent to the controllable sources to adjust their power output for damping of frequency deviations following a disturbance.

As shown in Fig. 2.27 the status of the generator and load agents is obtained and transmitted to MGCC. On the basis of the received information, MGCC will take decision and give on/off signals via UDP/IP to the circuit breakers. The MGCC produces the decision signals based on the following rules:

Rule 1. If the contribution of RESs (wind + solar) is higher than the load $\left(\mathrm{P}_{\mathrm{L} 0}\right)$ in steady state, the fuel cell and diesel engine generator should not contribute any power to the microgrid however the aqua-electrolyser will function. This is because there is excess power from the renewable sources in the microgrid and should be stored in the form of hydrogen.

Rule 2. If the contribution of RESs (wind + solar) is lower than the load in steady state, the aqua-electrolyser should not function. The fuel cell and diesel engine generator should start generating power and add to the microgrid.

Rule 3. If frequency deviation signal is not zero, and wind is generating more than $50 \%$ of its capacity, wind output should be reduced and SMES should inject its stored power to the system.

Rule 4. If frequency deviation signal is not zero, and PV is generating more than $30 \%$ of its capacity, its output should be reduced and SMES should inject stored power to the system.

Rule 5. In case of any cyber intrusion detection, the defense strategy and the proposed control algorithms, which will be described in the following sections, should be employed.

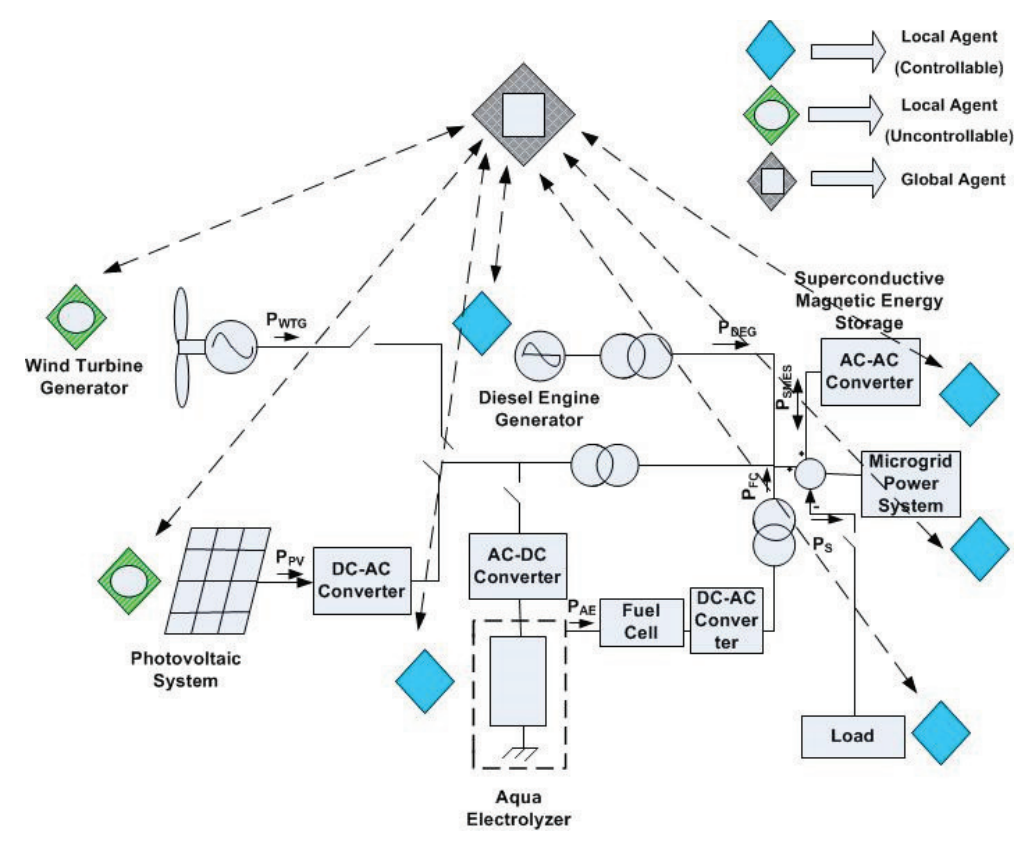

Fig. 2.26. Multi agent microgrid system for LFC. 


\section{State feedback LQR control design}

One of the approaches that is used in MGCC of Fig. 2.27 for tuning the control parameters of the controllable sources is LQR. $\mathrm{LQR}$ is a control scheme that gives the best possible performance with respect to a given performance criterion. For using this method we have to come up with the state space model of the system. State space model of the microgrid is shown in Eq. (2.30)

$$
\begin{aligned}
& \dot{X}=A X+B U+\Gamma P_{d} \\
& Y=C X
\end{aligned}
$$

where $\mathrm{X}, \mathrm{U}, \mathrm{P}_{\mathrm{d}}$ and $\mathrm{Y}$ are the state, control, disturbance and output vectors respectively. The system matrices: A (state matrix), $\mathrm{B}$ (input matrix), $\Gamma$ (disturbance matrix), and $\mathrm{C}$ (output matrix) are derived based on the microgrid parameters.

The state vector is

$$
X=\left[\Delta f \Delta P_{d g g} \Delta P_{d g t} \Delta P_{f c} \Delta P_{a e} \int \Delta f d t\right]^{T}
$$

The control vector is:

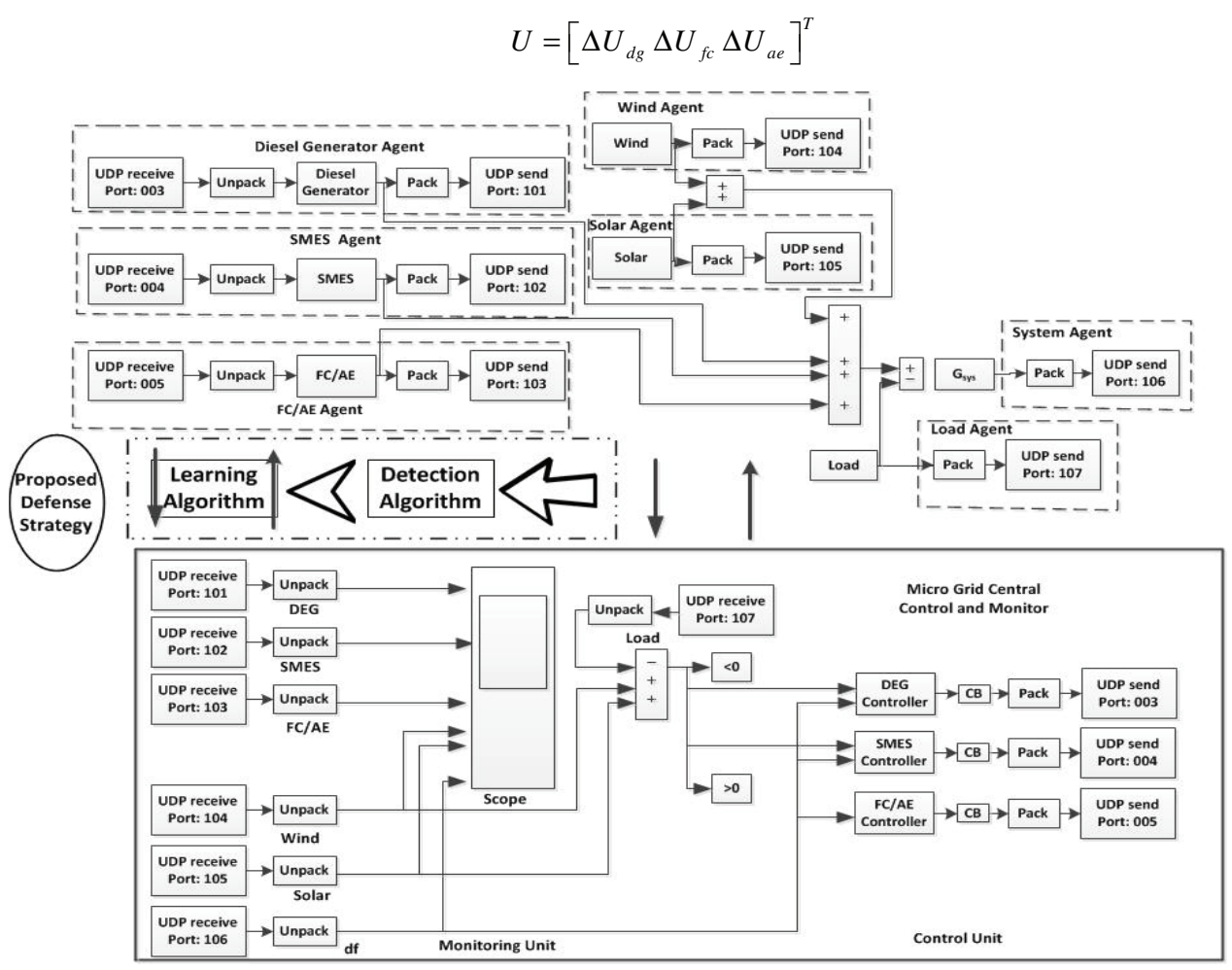

Fig. 2.32. Microgrid Central Control (MGCC) scheme.

To remove the disturbance vector we redefine the state variable set and control variable set as $\tilde{X}=\dot{X}, \tilde{U}=\dot{U}$. Therefore, we can find the optimal control strategy such that the cost function below is minimized.

$$
J_{L Q R}=\frac{1}{2} \int_{0}^{\infty}\left(\dot{X}^{T} Q \dot{X}+\dot{U}^{T} R \dot{U}\right)
$$

where

$$
\begin{gathered}
U=-K X \\
K=-R^{-1} B^{T} P
\end{gathered}
$$


KESHTKAR: MODELING AND CONTROLLING A HYBRID MULTI-AGENT BASED MICROGRID IN PRESENCE OF DIFFERENT PHYSICAL AND CYBER COMPONENTS

For finding P, Algebraic Riccuti Equation should be solved:

$$
A^{T} P+P A-P B R^{-1} B^{T} P+Q=0
$$

As it can be seen, different set of $\mathrm{Q}$ and $\mathrm{R}$ will results in different control design. Therefore, there is not a unique solution for this problem. In order to find the best set of $\mathrm{Q}$ and $\mathrm{R}$, the optimization is done to design $\mathrm{Q}$ and $\mathrm{R}$. Then LQR with this set of parameters is used to minimize the frequency deviation due to the load and generation changes in the microgrid system. The best $\mathrm{Q}$ and $\mathrm{R}$ based on the PSO algorithm in one sample situation are:

$$
\begin{aligned}
\mathrm{Q} & =\left[\begin{array}{cccccc}
4699 & 0 & 0 & 0 & 0 & 0 \\
0 & 1000 & 0 & 0 & 0 & 0 \\
0 & 0 & 1000 & 0 & 0 & 0 \\
0 & 0 & 0 & 1200 & 0 & 0 \\
0 & 0 & 0 & 0 & 7000 & 0 \\
0 & 0 & 0 & 0 & 0 & 1000
\end{array}\right] \\
R & =\left[\begin{array}{cccc}
0.09094 * 10^{3} & 0 & 0 \\
0 & 0.80066 * 10^{3} & 0 \\
0 & 0 & 1.50035 * 10^{3}
\end{array}\right]
\end{aligned}
$$

\section{Dual Heuristic Dynamic Programming (DHP)}

Another proposed approach which is used by controllable sources in MGCC of Fig. 2.27 is DHP which classified in ADP family. The Adaptive Dynamic Programming method is a type of Adaptive Critic Design (ACD) technique which consists of three neural networks: a model network, an action network, and a critic network. The model network can identify the time-varying dynamics of the nonlinear power system. The critic network, which approximates the control objective, trains the nonlinear action network to create an optimal control mapping between the plant feedbacks and control actions. ACDs methods have been successfully employed in different areas such as aircraft landing control [69], vibration isolation control [70], inverted pendulum system [71], etc. In this section, the objective function of the control methods is minimizing the deviation of frequency due to load and wind power variations as well as cyber attacks.

\section{D.1. Recurrent Neural Networks for Adaptive Critic Designs (ACDs)}

In this section, the Dual Heuristic dynamic Programming (DHP), which is a technique in the ACD family, is used for the frequency control purpose. Fig. 2.28 shows the schematic diagram of DHP where the critic network directly estimates $\lambda(\mathrm{k})$, the gradient of $\mathrm{J}(\mathrm{k})$ (the cost-to-go control objective) with respect to the plant outputs.

It should be noticed that in all materials of part $\mathrm{D}, \mathrm{u}(\mathrm{k})$ is the power deviation of the controllable sources $\left(\Delta P_{c}\right)$ as the input of the power plant, $\mathrm{y}(\mathrm{k})$ is the frequency deviation $(\Delta f)$ is the output of the power system model, and $\mathrm{J}(\mathrm{k})$ is the Area Control Error (ACE), which is used as the control objective ( $\beta$ is a weighting factor).

$$
A C E=\Delta f+\beta \Delta P_{c}
$$

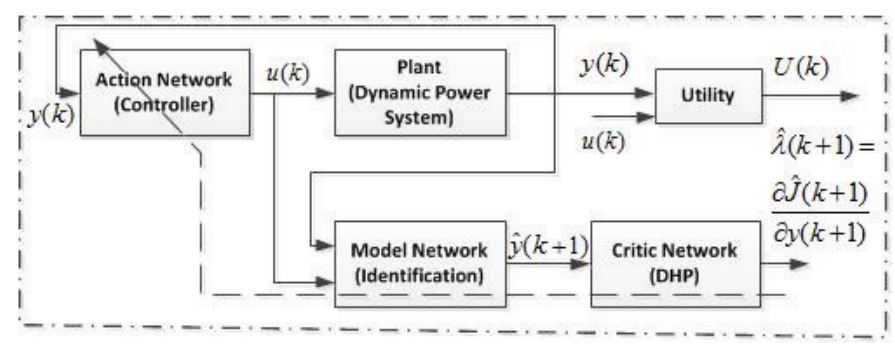

Fig. 2.28. Schematic diagram of DHP neurocontrol design with RNNs 
Fig. 2.29 shows the implementation of DHP in frequency control of the MGCC in the modeled Microgrid.

\section{D.2. Training of a DHP-based Neurocontroller using Recurrent Neural Networks (RNNs)}

The training of a DHP-based neurocontroller is done in two steps in this section: 1) offline training of the model network; and 2) online training of all three networks [71]. In the following section, $\mathrm{W}_{\mathrm{im}}, \mathrm{W}_{\mathrm{sm}}$, and $\mathrm{W}_{\mathrm{om}}$ are weights for the model network, $\mathrm{W}_{\mathrm{ic}}$, $\mathrm{W}_{\mathrm{sc}}$, and $\mathrm{W}_{\mathrm{oc}}$ are weights for the critic network, $\mathrm{W}_{\mathrm{ia}}, \mathrm{W}_{\mathrm{sa}}$, and $\mathrm{W}_{\mathrm{oa}}$ are weights for the action network.

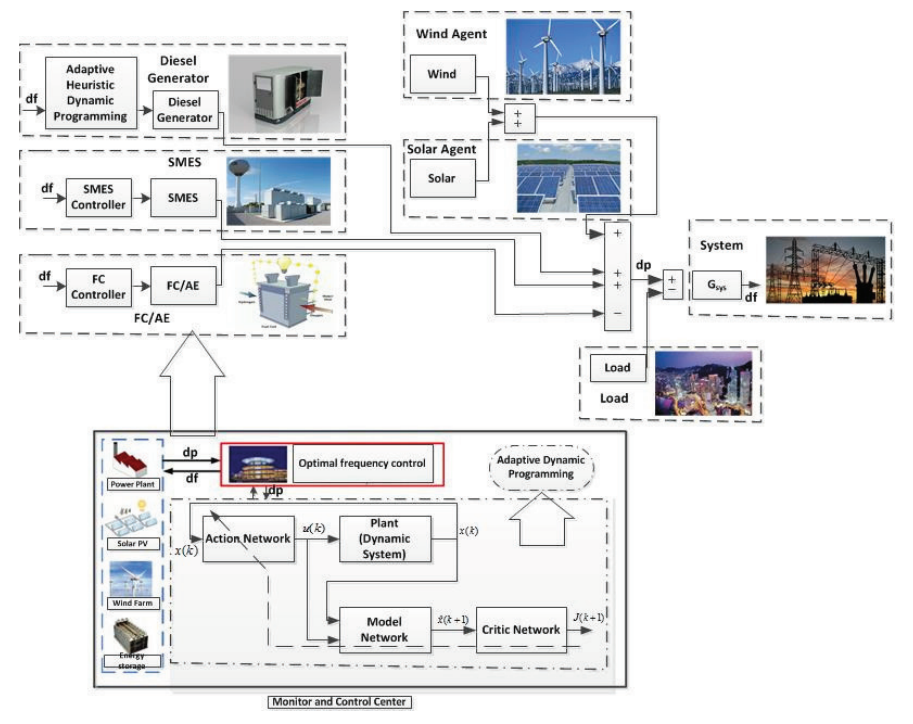

Fig. 2.29. Simulation model of the studied Microgrid

\section{D.3. Offline Training of Model Network}

The plant is first operated in an open-loop action network. Random signals are injected into the plant inputs, $\mathrm{u}(\mathrm{k})$, over a wide operating range. The plant responses, $\mathrm{y}(\mathrm{k})$, are recorded along with $\mathrm{u}(\mathrm{k})$. Having values of the recorded $\mathrm{u}(\mathrm{k})$ and $\mathrm{y}(\mathrm{k})$ at different operating points, the model network is trained offline to minimize the following mean squared error (MSE):

$$
\begin{gathered}
E_{m}(k)=\left\|e_{m}(k)\right\|^{2}=\|y(k)-\hat{y}(k)\|^{2} \\
M S E=\text { average }\left[\sum_{\substack{\text { operating } \\
\text { points }}} \sum_{k} E_{m}(k)\right]
\end{gathered}
$$

where $\hat{y}(k)$ is the one-step delay of the model network output. In other words, the model network is trained to identify the plant dynamics.

The model network is initialized with small random weights. During the training step, the one-step model prediction error and error gradients are obtained based on the below equations,

$$
\begin{aligned}
& \frac{\partial E(k)}{\partial W_{o}}=[\nabla g] \cdot \frac{\partial E(k)}{\partial o(k)} \cdot s(k)^{\prime} \\
& \frac{\partial E(k)}{\partial W_{s}}=[\nabla f] W_{o}^{\prime} \cdot[\nabla g] \cdot \frac{\partial E(k)}{\partial o(k)} \cdot s(k-1)^{\prime} \\
& \frac{\partial E(k)}{\partial W_{i}}=[\nabla f] W_{o}^{\prime} \cdot[\nabla g] \cdot \frac{\partial E(k)}{\partial o(k)} \cdot i(k)^{\prime}
\end{aligned}
$$

where $\mathrm{i}(\mathrm{k})$ is the input vector, $\mathrm{o}(\mathrm{k})$ is the output vector, $\mathrm{s}(\mathrm{k})$ is the $\mathrm{RNN}$ internal state vector, $f(\cdot)$ and $g(\cdot)$ are activation functions. $\mathrm{E}(\mathrm{k})$ is an error measurement related the RNN outputs. More details on calculating this error for the model, critic and action 
KESHTKAR: MODELING AND CONTROLLING A HYBRID MULTI-AGENT BASED MICROGRID IN PRESENCE OF DIFFERENT PHYSICAL AND CYBER COMPONENTS

networks are described in the rest of this section. $[\nabla g]$ and $[\nabla f]$ are diagonal matrices containing the gradients of $g(\cdot)$ and $f(\cdot)$, respectively.

The model weights are then updated based on the below equations. The model training continues until the MSE calculated in the testing step drops below a specific value.

$$
\begin{aligned}
& W_{o}(k+1)=W_{o}(k)-r_{o} \frac{\partial E(k)}{W_{o}} \\
& W_{s}(k+1)=W_{s}(k)-r_{s} \frac{\partial E(k)}{W_{s}} \\
& W_{i}(k+1)=W_{i}(k)-r_{i} \frac{\partial E(k)}{W_{i}}
\end{aligned}
$$

\section{D.4. Online Training of Model, Critic, and Action Networks}

After the offline training, the model network is used to provide system-wide cross-coupling sensitivity signals, $\partial \mathrm{y}(\mathrm{k}+1) / \partial \mathrm{u}(\mathrm{k})$, over a wide operating range. When the critic and action networks are trained, the model weights are continuously updated with a small learning rate to ensure tracking of new operating conditions [72].

An RNN DHP critic network is trained online to approximate $\lambda(k+1)$, the partial derivative of $\mathrm{J}(\mathrm{k}+1)$ with respect to $\mathrm{y}(\mathrm{k}+1)$, by minimizing the following error:

$$
\begin{aligned}
E_{c}(k)= & \left\|e_{c}(k)\right\|^{2} \\
e_{c}(k)= & \hat{\lambda}(k)-\left\{\frac{\partial U(k)}{\partial y(k)}+\left[\frac{\partial u(k)}{\partial y(k)}\right] \frac{\partial U(k)}{\partial u(k)}\right. \\
& \left.+\gamma\left[\frac{\partial \hat{y}(k+1)}{\partial y(k)}+\frac{\partial \hat{y}(k+1)}{\partial u(k)} \frac{\partial u(k)}{\partial y(k)}\right] \hat{\lambda}(k+1)\right\}
\end{aligned}
$$

The training of the critic network is done online along with the action network in the loop. The training starts with a small discount factor $\gamma$. As the critic weights converge, the discount factor is gradually increased.

The optimal control objective of the controller is to minimize $\mathrm{J}(\mathrm{k}$ ) (or ACE) at every time step k, which is accomplished by training an RNN action network. The action network is trained to approximate the optimal control law by minimizing (2.44)

$$
\begin{aligned}
& E_{a}(k)=\left\|\frac{\partial \hat{J}(k)}{\partial u(k)}\right\|^{2} \\
& \frac{\partial \hat{J}(k)}{\partial u(k)}=\frac{\partial U(k)}{\partial u(k)}+\gamma\left[\frac{\partial \hat{y}(k+1)}{\partial u(k)}\right]^{\prime} \hat{\lambda}(k+1)
\end{aligned}
$$

During the training, when $\mathrm{E}_{\mathrm{a}}(\mathrm{k})$ becomes zero, $\mathrm{u}(\mathrm{k})$ is the optimal control action that minimizes $\mathrm{J}(\mathrm{k})$ in the local region. Global optimal is obtained by exposing the RNN action network to different system conditions.

At sampling step k, the output-to-input derivatives of the model, critic, and action networks are given by

$$
\frac{\partial o(k)}{\partial i(k)}=[\nabla g] W_{o} \cdot[\nabla f] W_{i}
$$

The model prediction error is given by (2.39), and the model error gradients are given by (2.41). The utility derivatives can be obtained based on the utility formulation, which is usually a function of the plant outputs and the control effort. Assuming a linear output activation function for the critic network, the DHP critic network output at time k is calculated by

$$
\hat{\lambda}(k+1)=W_{o c} S_{c}(k)=W_{o c} f_{c}\left[W_{i c} \hat{y}(k+1)+W_{s c} S_{c}(k-1)\right]
$$


where $s_{c}(k)$ is the internal state vector of the critic network, and $f_{c}(\cdot)$ is the hidden layer activation function of the critic network. When calculating the critic error, given by (2.43), and it should be based on the same set of critic weights. With a truncated depth of one, the critic error gradient with respect to $\mathrm{W}_{\text {oc }}$ is given by

$$
\frac{\partial E_{c}(k)}{\partial W_{o c}}=2 \cdot e_{c}(k)^{\prime} \cdot \frac{\partial \hat{\lambda}(k)}{\partial W_{o c}}-2 \gamma e_{c}(k)^{\prime}\left[\frac{\partial \hat{y}(k+1)}{\partial y(k)}\right] \cdot \frac{\partial \hat{\lambda}(k+1)}{\partial W_{o c}}
$$

Where

$$
\frac{\partial \hat{y}(k+1)}{\partial y(k)}=\frac{\partial \hat{y}(k+1)}{\partial y(k)}+\frac{\partial \hat{y}(k+1)}{\partial u(k)} \frac{\partial u(k)}{\partial y(k)}
$$

The critic error gradient with respect to $\mathrm{W}_{\mathrm{sc}}$ is given by

$$
\frac{\partial E_{c}(k)}{\partial W_{s c}}=2 \cdot e_{c}(k)^{\prime} \cdot \frac{\partial \hat{\lambda}(k)}{\partial W_{s c}}-2 \gamma e_{c}(k)^{\prime}\left[\frac{\partial \hat{y}(k+1)}{\partial y(k)}\right] \cdot \frac{\partial \hat{\lambda}(k+1)}{\partial W_{s c}}
$$

The critic error gradient with respect to $\mathrm{W}_{\text {ic }}$ is given by

$$
\frac{\partial E_{c}(k)}{\partial W_{i c}}=2 \cdot e_{c}(k)^{\prime} \cdot \frac{\partial \hat{\lambda}(k)}{\partial W_{i c}}-2 \gamma e_{c}(k)^{\prime}\left[\frac{\partial \hat{y}(k+1)}{\partial y(k)}\right] \cdot \frac{\partial \hat{\lambda}(k+1)}{\partial W_{i c}}
$$

After obtaining the model output-to-input derivatives and critic outputs, the action error is given by (2.51).

$$
\begin{aligned}
& E_{a}(k)=\left\|\frac{\partial \hat{J}(k)}{\partial u(k)}\right\|^{2} \\
& \frac{\partial \hat{J}(k)}{\partial u(k)}=\frac{\partial U(k)}{\partial u(k)}+\gamma\left[\frac{\partial \hat{y}(k+1)}{\partial u(k)}\right] \hat{\lambda}(k+1)
\end{aligned}
$$

With a truncated depth of one, the action error gradients with respect to its weights can be obtained using (2.41).

During each sampling step, the weights of both the action and critic networks are updated based on (2.42). The online training continues until both the critic and action errors converge to zero.

\subsection{Overview of Particle Swarm Optimization (PSO)}

The control parameters of the generator systems in PI mode and Q and R in LQR control mode of the studied power microgrid system are optimized by Particle Swarm Optimization (PSO) Algorithm to minimized the frequency oscillations and make the comparisons reasonable. Therefore, this algorithm is discussed in this section. This algorithm was discussed in previous sections for other problems, it is needed to be formulated for the particular problem of this part.

PSO is a multi-agent search approach, which traces its evolution to the motion of a flock of birds searching for food [73]. It uses a number of particles that are called a swarm. Each particle traverses the search space searching for the global minimum (or maximum). In a PSO system, particles fly within a multi-dimensional search space. During flight, each particle sets its position based on its own experience and the experience of neighboring particles. Hence, it makes use of the best position encountered by itself and its neighbors. Similarly, the swarm direction and speed of a particle is determined by the history experience obtained by itself as well as a set of its neighboring particles [15].

Each particle has different control variables such as generators and load power output affecting frequency deviation in each iteration. Let us consider $p$ and $s$ as particle position and its flight speed in a search space, respectively. The best position of a particle in each step is recorded and represented as $\mathrm{P}_{\text {best. }}$ The best particle's index among all the particles in the group is considered 
KESHTKAR: MODELING AND CONTROLLING A HYBRID MULTI-AGENT BASED MICROGRID IN PRESENCE OF DIFFERENT PHYSICAL AND CYBER COMPONENTS

as $\mathrm{G}_{\text {best. }}$ The convergence of PSO is ensured by use of a constriction function. Finally, the modified velocity and position of each particle can be calculated as shown in (2.52) and (2.53):

$$
\begin{gathered}
s_{d+1}=k *\left(\gamma^{*} v_{d}+a c_{1} \cdot \operatorname{rand}() *\left(P_{b e s t}-P_{d}\right)+a c_{2} * \operatorname{rand}() *\left(G_{b e s t}-P_{d}\right)\right) \\
P_{d+1}=P_{d}+s_{d+1}
\end{gathered}
$$

where $\mathrm{d}$ is the index of iteration, $P_{d}$ is the current particle's position at the d-th iteration, $s_{d}$ is the particle's speed of at d-th iteration, $\gamma$ is inertia weight factor, $a c_{1}$ and $a c_{2}$ are acceleration constants, rand() is a uniform random value in the range [0,1] [75], and $\mathrm{k}$ is the constriction factor which is a function of $a c_{1}$ and $a c_{2}$ according to (2.54):

$$
k=\frac{2}{\mid 2-a c-\sqrt{a c_{2}-4 a c \mid}}
$$

where $a c=a c_{1}+a c_{1}$ and $a c \succ 4$. Appropriate choice of inertia weight, $\gamma$, makes a balance between global and local explorations. In general, $\gamma$ is calculated according to (2.55):

$$
\gamma=\gamma_{\max }-\frac{\gamma_{\max }-\gamma_{\min }}{\text { iter }_{\max }} \times \text { iter }
$$

Where iter $r_{\max }$ is the maximum number of iterations, and iter is the number of the iterations up to current stage.

In the above procedures, the particle speed is bounded by a maximum value, $\mathrm{S}^{\max }$. This value defines the fitness by which regions are to be searched between the present and the target position. This limit increases the local exploration of the problem space. It also realistically simulates the changes of human learning. If $\mathrm{S}^{\max }$ is too big, particles may ignore good solutions. On the other hand, a small $S^{\max }$ may cause particles not to sufficiently explore beyond local solutions. The latter may leads the PSO to fall into local minimum. In many optimizations with PSO, $\mathrm{S}^{\max }$ was often set at $10 \%-20 \%$ of the dynamic range of the variable on each dimension [15].

As it was mentioned, the proposed control approaches are going to be tested in presence of cyber attacks on the measurement and communication signals of the modeled multi-agent based microgrid. Therefore, detection and learning algorithm which is used to detect the intrusion and implementing the defense strategy based on the proposed control approaches is discussed in the next part.

\subsection{Detection and Learning Algorithm}

The resilience of the microgrid power system becomes susceptible as the power grid turns out to be vulnerable to the cyber attacks than the internal errors of operations [76]. Thus, it is crucial to carry out the analysis of vulnerability incurred by the cyber attacks on the multi-agent based microgrid power system and defense against the impacts due to the attacks. In this part, detection and learning algorithms shown in Fig. 2.27 which are used in this work in case of cyber attacks in the microgrid system are discussed.

\section{A. Detection method}

Based on the characteristics of the sensitive signals, attack detection strategies are developed [70]. Frequency signal of the power system can be oscillated due to power changes in the system. These oscillations are usually due to power mismatch in the system and the controllers try to damp them in a reasonable period of time. Basically, frequency deviation due to normal changes 
in the system has a level of smoothness. However, when there is a kind of attack on the frequency control system, this smoothness can be deteriorated. Therefore, by checking the continuousness and rate of the slope changes of the frequency response of the system, the happening of a cyber attack can be detected.

$$
\begin{aligned}
& \alpha_{1}=\operatorname{Arctan}(\Delta f(z)-\Delta f(z-1)) \\
& \alpha_{2}=\operatorname{Arctan}(\Delta f(z-1)-\Delta f(z-2)) \\
& \alpha_{3}=\alpha_{1} \cdot \alpha_{2}
\end{aligned}
$$

where $\Delta f(z)$ is the frequency deviation at step time $\mathrm{z}$, and $\alpha_{3}$ is the input signal of detection module which decides about the integrity of the signal and existence of the attack based on pre-defined thresholds to avoid detecting normal changes in the system.

This attack detection method is a general method which is usable for most of the attacks on the modeled microgrid. However, detailed detection strategy is presented in Fig. 2.30 which is used for the kinds of intrusions (replay and data loss) which are discussed and simulated in simulation section.

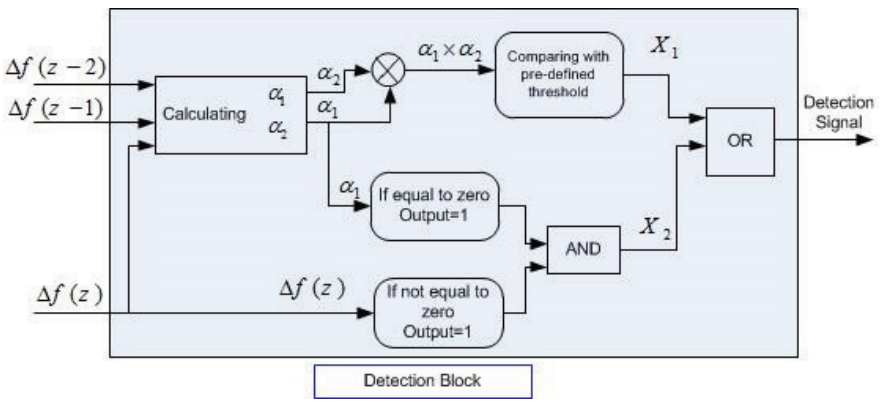

Fig. 2.30. Schematic of the proposed detection strategy

As it can be seen in Fig. 2.30, $\alpha_{1}$ and $\alpha_{2}$ are generated by using two consecutive steps of the frequency deviation signals. Then, the product of these two signals is compared to a pre-defined threshold to detect a replay attack. In case of data loss attack, the frequency measurement will be lost and the reported value will be remained constant for the period of the attack. So the steadiness of the frequency signal should be analyzed to detect the interruptions created by the attack. Therefore, if the consecutive frequency deviations signal $\left(\alpha_{1}\right)$ is zero and the frequency error $(\Delta f(z))$ has a non-zero value, that means the data of the frequency measurement output is lost. $X_{1}$ and $X_{2}$ are the command signals which are transmitted as the detection signal to the MGCC to be analyzed by the global agent for any possible attack in the system.

\section{B. Learning method}

After the occurrence of a cyber attack on frequency signal of the system, the controllers will act based on the incorrect signal formed by the attack. This may cause the frequency response to become more oscillatory and make the system go to instability. Therefore, based on different frequency oscillations, the controllers should be trained to set the control parameters to adjust the control variables such as generating and load unit output power (load and generator shedding in case of high frequency changes) in order to bring back the frequency to nominal value in case of cyber attacks. In other words, the learning algorithm is designed in MGCC to be used after each attack detection, to set the controller parameters (for example Q and R), regardless of incorrect measurement signal due to the attack.

The Reinforcement Learning (RL) algorithm is used to learn a closed-loop control law and able to damp large oscillations [77]. Since there are lots of different oscillations in frequency of the system caused by variety of sources, which is aimed to be controlled, we need to check the robustness of the control law obtained after training. Thus, after convergence of the RL algorithm for one particular oscillation, we will assess the results of the control gains on other scenarios different from the one for this learning. 
KESHTKAR: MODELING AND CONTROLLING A HYBRID MULTI-AGENT BASED MICROGRID IN PRESENCE OF DIFFERENT PHYSICAL AND CYBER COMPONENTS

The learning period is partitioned into different scenarios. Each scenario starts with the power system being at rest with no power mismatch and frequency oscillation. The load change happens at a random value and frequency starts deviating. The simulation then proceeds using the proposed controllers to damp the frequency oscillations and the controller is trained to choose the optimal control parameters, to make the system stable quickly. Several learning scenarios are generated for different oscillation scenarios to train the control parameters for different frequency oscillations. Therefore, after detecting the cyber attack MGCC uses the learning algorithm, which is explained and formulated in the next part, to come up with the best control parameters based on the proposed approaches to damp the frequency deviations and it avoids using the fake signal and deteriorating the frequency response of the system.

\section{Learning algorithm}

The objective is to maximize the sum of returned rewards over time. The return reward here is the inverse of ACE signal which is calculated based on (2.38). The expected sum of discounted rewards is defined by [77]

$$
R=\sum_{k=0}^{\infty} \gamma^{k} r_{t+k+1}
$$

where $0<\gamma<1$ is a discount factor, which gives the maximum importance to the recent rewards. The expected return (reward) when starting at state $\mathrm{x}_{\mathrm{t}}$ while following policy pi(x, a) is called value function.

$$
V^{\pi}(x)=E_{\pi}\left\{\sum_{k=0}^{\infty}\left[\gamma^{k} r_{t+k+1} \mid x_{t}=x\right]\right\}
$$

Policy is the way in which the agent maps the power system states, shown in (2.31), to the frequency control actions by the proposed controllers. The optimal policy is the one that maximizes the value function. Sometimes, the value function is replaced by action value which is the expected discounted reward while starting at state $\mathrm{x}_{\mathrm{t}}$ and taking action $\mathrm{a}_{\mathrm{t}}$

$$
Q^{\pi}(x, a)=E_{\pi}\left\{\sum_{k=0}^{\infty}\left[\gamma^{k} r_{t+k+1} \mid x_{t}=x, a_{t}=a\right]\right\}
$$

After detection of the different attack cases on the cyber parts of the multi-agent microgrid, the above learning and defense strategy is applied using the proposed control algorithms described in this Section. In the next section the analysis on the simulation results is discussed. 


\section{III- Simulation Results AND ANAlysis}

\section{MICROGRID MODELING}

Simulation results related to the configuration described in part 1.1 of section II are shown in Fig. 2.31. These results under various operating conditions are, respectively, analyzed in the following cases.

1) Base Case: During $0 \mathrm{~s}<t<200 \mathrm{~s}$, the average wind speed $V_{w}$ is around $7.5 \mathrm{~m} / \mathrm{s}$, and the total average power generated by three WTGs, $P_{W T G}$, is less than 0.3 p.u. Since low power is generated by three WTGs, the DEG starts injecting power to the system at $t$ $=0$ s to supply $P_{D E G}$ of around 0.9 p.u. $P_{p v}$ is varied correspond to solar energy. No surplus energy can be stored in the SMES due to low power generation condition and system frequency deviation $\Delta f$ is varied around zero.

2) Sudden Wind Speed and Load Drop: When $200 \mathrm{~s}<t<250 \mathrm{~s}, V_{w}$ suddenly drops to a low-wind level and $P_{s}$ suddenly drops from 1.0 to 0.5 p.u. at $t=200 \mathrm{~s}$. Accordingly, $P_{D E G}$ also drops from around 0.2 to 0 p.u. $P_{p v}$ is varied correspond to solar energy. The system frequency deviation $\Delta f$ slightly rises above zero since total power generation is slightly higher than load demand.

3) Sudden Wind Speed and Load Rise: For $250 \mathrm{~s}<t<300 \mathrm{~s}, V_{w}$ suddenly rises to a high-wind level and $P_{s}$ suddenly rises from 0.5 to 1.0 p.u. at $t=250 \mathrm{~s}$. Since enough power is generated by WTGs, the DEG produces low amount of power generation. Owing to high-power generation of WTGs, the surplus power generation can be stored in the SMES through associated power converters. $P p v$ is still varied correspond to solar energy. The response of $\Delta f$ is severely varied when it is compared with the ones under the base case and the low wind-speed condition.

In this configuration, the SMES besides wind and photovoltaic generation timely react to the load change and wind speed disturbances. In overall, due to fast-response ESS for LFC the transient response of the system is improved.

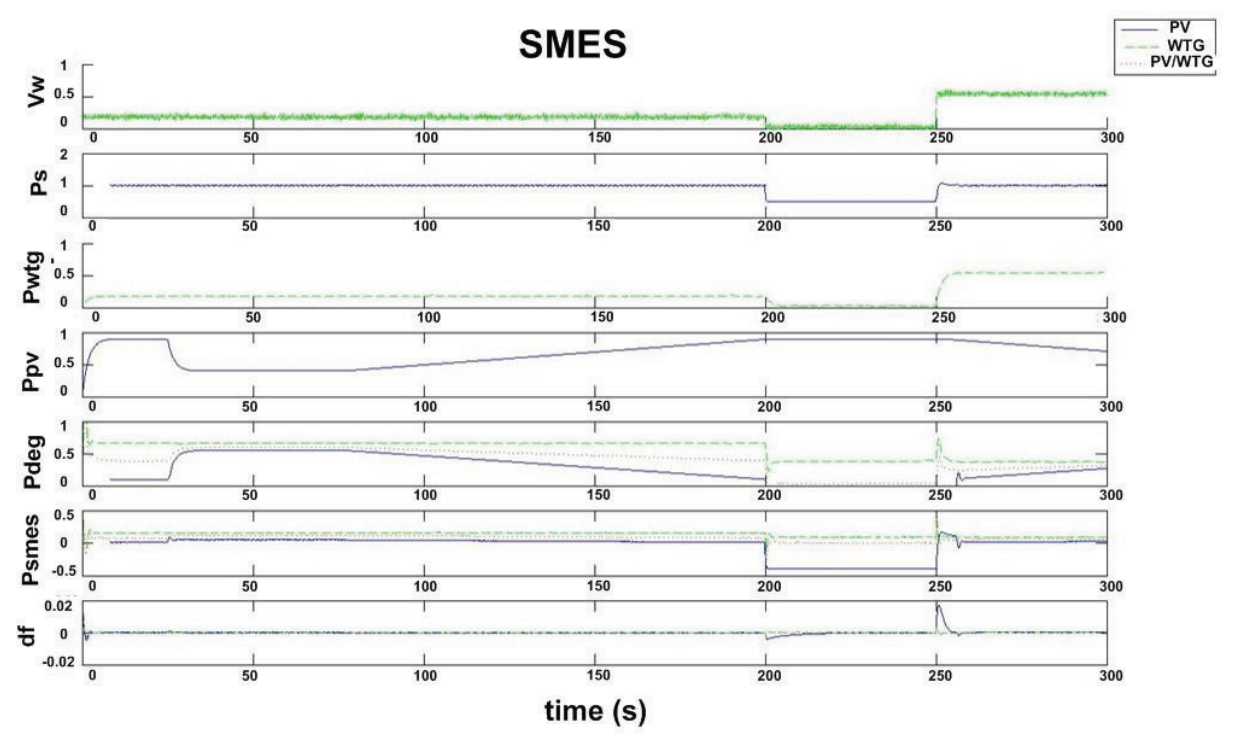

Fig. 2.31. Power flow and frequency response of the one of the configurations. 


\section{KESHTKAR: MODELING AND CONTROLLING A HYBRID MULTI-AGENT BASED MICROGRID IN PRESENCE OF DIFFERENT PHYSICAL AND CYBER COMPONENTS}

\section{MICROTURBINE IN MICROGRID}

\subsection{Case Study}

Case I

The first system that is assumed to be analyzed isolated from distribution system includes turbine and its controllers, permanent magnet synchronous machine and power conditioning models. The modeling is described in part 2.1 of section II. The parameters of different part of the system are reported in Table 2.III and 2.IV. The load change which the controller is designed for it is described in part 2.2 of section II. There is the possibility for the modern controllers with the ability of changing parameters to have a look-up table for 24-hour load changes and optimize the parameters simultaneously.

\section{Case II}

The second case study is a hybrid power generation/energy storage system shown in Fig. 2.32. This system consists of wind generators, photovoltaic, diesel generators, and superconductive magnetic energy storage. The modeling of this microgrid was presented in part 1.1. The proposed MT model is added to this system to study its application in transient control problems. Power supplied to the load $\mathrm{P}_{\mathrm{s}}$ is the sum of output power from wind turbine generators $P_{w}$, diesel generators $P_{g}$, photovoltaic (PV) generation $P_{p v}$, Microturbine $P_{m t}$, and negative input power to Superconducting Magnetic energy Storage (SMES) $\mathrm{P}_{\text {smes }}$ given by

$$
P_{s}=P_{w}+P_{g}+P_{p v}+P_{m t}-P_{s m e s}
$$

\subsection{Simulation Results}

\section{Case I}

In order to verify the proposed approach, the simulation results for every steps of implementation of DE in the microturbine speed governor model are presented in Table 2.V for daily load change at $12 \mathrm{am}$. As mentioned, the weighting factor for each objective, designate the importance of its optimization. So the step1 has resulted the best condition for fuel consumption and the step2 has resulted the best response of the speed.

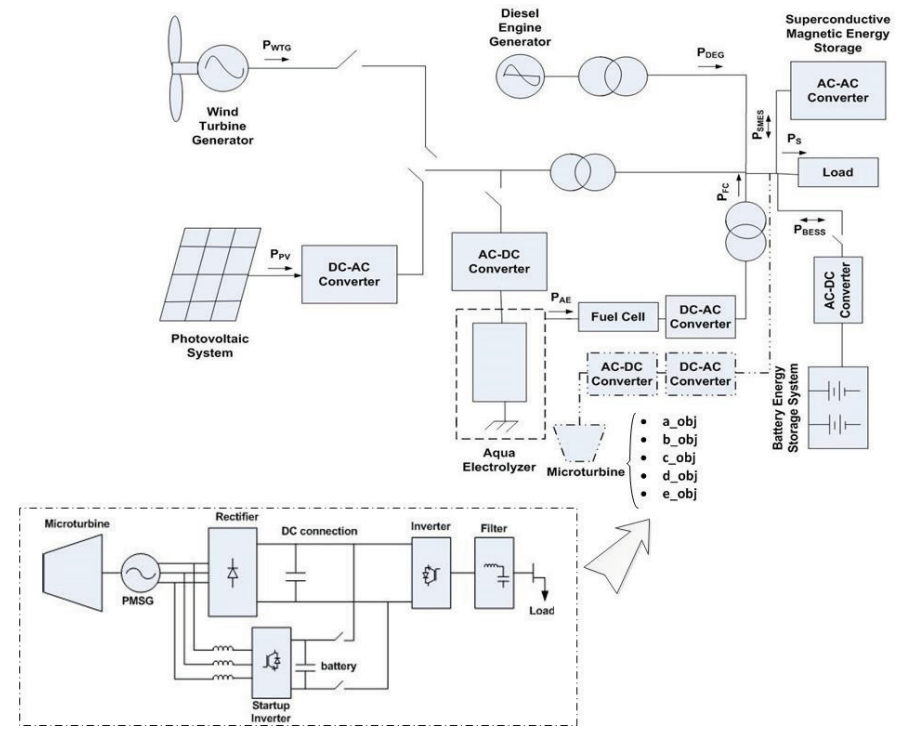

Fig. 2.32. Adding the proposed MT model to a hybrid Microgrid 
TABLE 2.III

The parameters of PMSM

In Table 2.VI, the results of the different weighting factor of the objectives is presented. The quality of the objectives are varied correspond to the weighting factors which are allocated to them. Several simulation results depict that the weighting factors shown in bold column are the most suitable ones for both objectives and will propose the optimized parameters of the speed governor. The best control parameters for this case are $\mathrm{a}=1, \mathrm{c}=1.5, \mathrm{~d}=3, \mathrm{e}=7$.

This simulation is carried out for different load changes of daily hours shown in Fig. 2.8 of part 2.1 of section II and the results are shown in Table 2.VII. The results show that bigger load changes, such as the one at $11 \mathrm{pm}$, cause larger optimum objectives and bigger weighting factor for speed deviation to get the multi-optimal solution.

\section{Case II}

\begin{tabular}{|c|c|}
\hline parameter & value \\
\hline $\mathrm{R}_{\mathrm{s}}$ & $2.875 \mathrm{ohm}$ \\
\hline No. of poles & 4 \\
\hline $\mathrm{L}_{\mathrm{d}}, \mathrm{L}_{\mathrm{q}}$ & $8.5 \mathrm{mH}$ \\
\hline$\lambda$ & $0.10396 \mathrm{wb}$ \\
\hline
\end{tabular}

TABLE 2.IV

The load parameters

\begin{tabular}{|c|c|}
\hline parameter & value \\
\hline Rated Power & $50 \mathrm{KW}$ \\
\hline Rated Voltage & $480 \mathrm{Vrms}$ \\
\hline Frequency & $50 \mathrm{~Hz}$ \\
\hline
\end{tabular}

TABLE 2.V

The simulation result of DE steps after daily load changing

\begin{tabular}{|l|c|c|}
\hline Objectives Steps & $\begin{array}{c}\text { Total fuel } \\
\text { consumption }(\mathrm{pu})\end{array}$ & $\begin{array}{c}\text { Speed variation } \\
(\mathrm{pu})\end{array}$ \\
\hline Step1 (min of 1st objective) & 0.8283 & - \\
\hline Step2 (min of 2nd objective) & - & $1.4559 * 10-6$ \\
\hline $\begin{array}{l}\text { Step3 (max of both } \\
\text { objectives) }\end{array}$ & 1.3050 & 5.7497 \\
\hline Step4 (multi-optimization) & 0.9684 & 0.0026 \\
\hline
\end{tabular}

In the second step of the simulations, the proposed model has been added to a hybrid microgrid consist of different renewable energy sources, DGs, and energy storages. Then by putting the modeled MT in the microgrid and using DE the optimal control parameters of the generating and storage units are calculated to minimize the frequency changes for the same load change which is used for MT modeling. The results of power flow and frequency are shown in Fig. 2.33 and 2.34.

TABLE 2.VI

The simulation result for different weighting factors

\begin{tabular}{|c|l|c|c|c|c|c|}
\hline \multirow{2}{*}{$\begin{array}{c}\text { Weighting } \\
\text { factor }\end{array}$} & $\begin{array}{l}\text { Total fuel } \\
\text { consumption } \\
\text { factor (C1) }\end{array}$ & 1 & 0 & 1 & $\mathbf{1}$ & 1 \\
\cline { 2 - 7 } & $\begin{array}{l}\text { Speed } \\
\text { variation } \\
\text { factor (C2) }\end{array}$ & 0 & 1 & 1 & $\mathbf{1 4}$ & 20 \\
\hline Total fuel consumption (pu) & 0.8283 & 1.2456 & 0.839 & $\mathbf{0 . 9 6 8 4}$ & 1.15 \\
\hline \multicolumn{2}{|l}{ Speed variation (pu) } & 2.539 & $1.456 * 10^{-6}$ & 0.589 & $\mathbf{0 . 0 0 2 6}$ & $2.4 * 10^{-5}$ \\
\hline
\end{tabular}
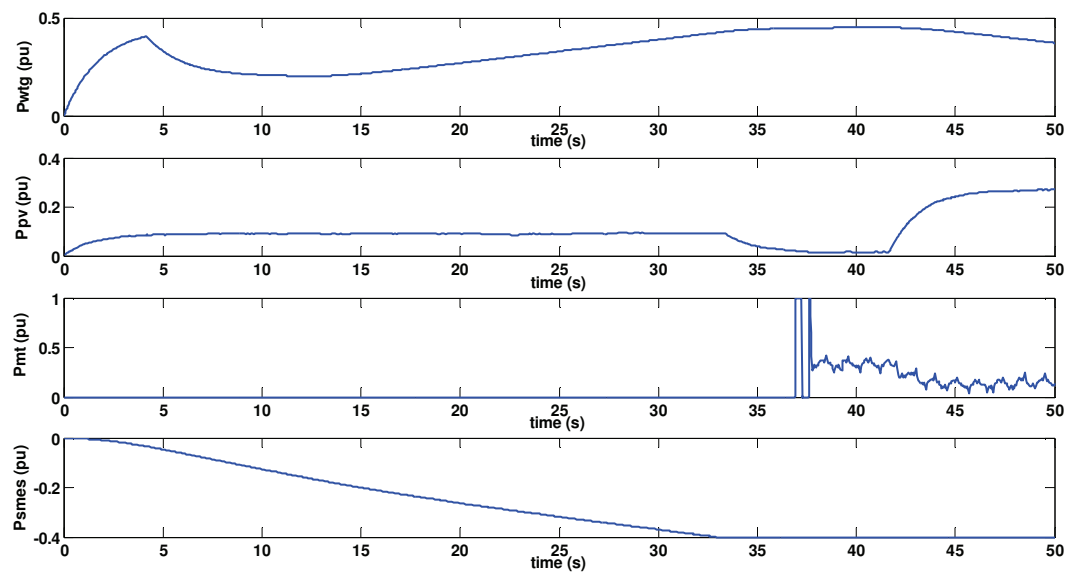

Fig. 2.33. Power flow results of the frequency control of the Microgrid 
KESHTKAR: MODELING AND CONTROLLING A HYBRID MULTI-AGENT BASED MICROGRID IN PRESENCE OF DIFFERENT PHYs

TABLE 2.VII

The simulation result for different daily hours' load change

\begin{tabular}{|c|c|c|c|c|c|c|}
\hline \multicolumn{2}{|c|}{ Daily hours } & $12 \mathrm{am}$ & $8 \mathrm{am}$ & $12 \mathrm{pm}$ & $6 \mathrm{pm}$ & $11 \mathrm{pm}$ \\
\hline $\begin{array}{c}\text { Weighting } \\
\text { factor }\end{array}$ & $\begin{array}{c}\text { Total fuel } \\
\text { factor (C1) }\end{array}$ & 1 & 1 & 1 & 1 & 1 \\
\cline { 2 - 7 } & $\begin{array}{c}\text { Speed variation } \\
\text { factor (C2) }\end{array}$ & 14 & 15 & 7 & 11 & 17 \\
\hline \multicolumn{2}{|c|}{ Total fuel consumption (pu) } & 0.9684 & 0.9864 & 0.7783 & 0.8403 & 0.9916 \\
\hline \multicolumn{2}{|c|}{ Speed variation (pu) } & 0.0026 & 0.0034 & 0.0012 & 0.0019 & 0.0044 \\
\hline
\end{tabular}

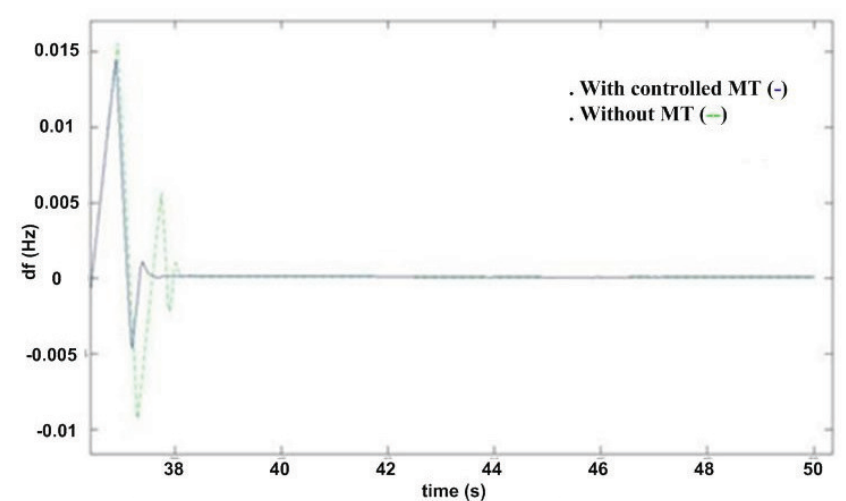

Fig. 2.34. Frequency results of the Microgrid with and without MT

As it can be seen from Fig. 2.33, Photovoltaic (PV) and Wind Turbine Generator (WTG) are working based on available renewable sources. The control center is designed in such a way that the fast-response MT works after considerable disturbance in the system. Therefore, it comes to picture after the load change. SMES absorbs the extra generated power in the system, especially renewable sources and MT. So it absorbs its maximum capacity after the load change. It can be concluded from Fig. 2.34 that frequency control is better when using MT in Microgrid due to the fast-acting characteristic of it. However, simulation time increases because of the extra time due to MT power conditioning model and also the delay added for optimal design of MT controller.

\section{PHEV Simulation Results}

\subsection{PHEV penetration}

Results are divided into different parts to analyze the effects of PHEV penetration on frequency response of the microgrid. The main two outputs are total power generating of PHEVs, and frequency deviation of the microgrid. These two outputs for a controlled PHEV system are shown in Fig. 2.35.
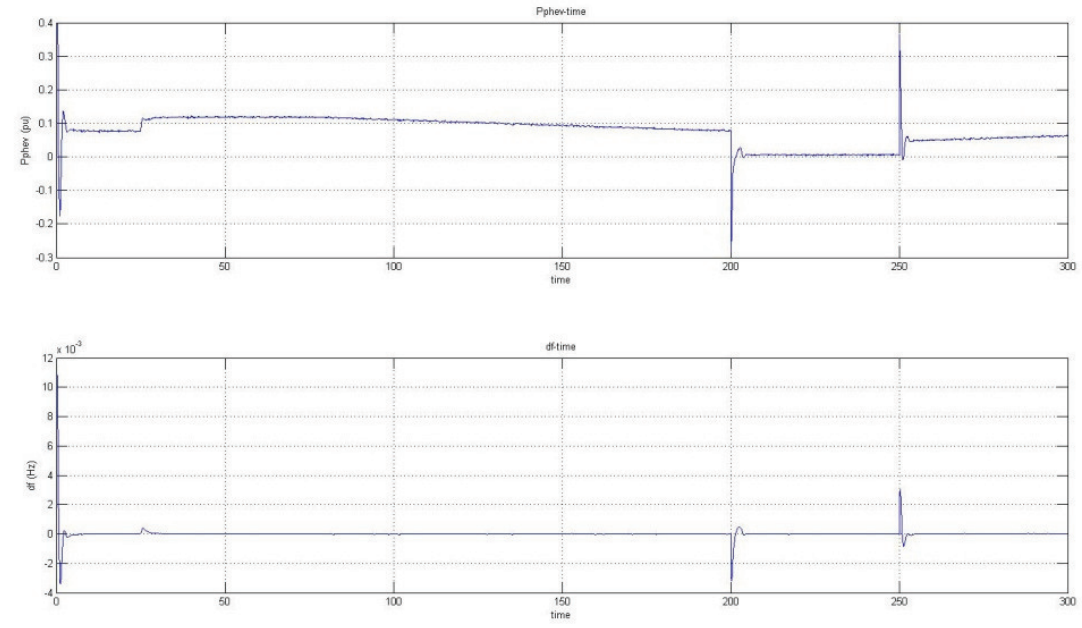

Fig. 2.35.Microgridoutputs for a controlled PHEV system 


\section{A. Effect of PHEV penetration in microgrid frequency}

PHEV used in the power system is optimally controlled and the results before and after PHEV penetration are compared with each other in Fig. 2.36. The first output is the total output power of PHEVs and the second one is frequency deviation of the microgrid due to wind and load changes. It is clear that using PHEV in case of frequency deviation of microgrid can be very useful and the frequency oscillation can be damped more effectively.
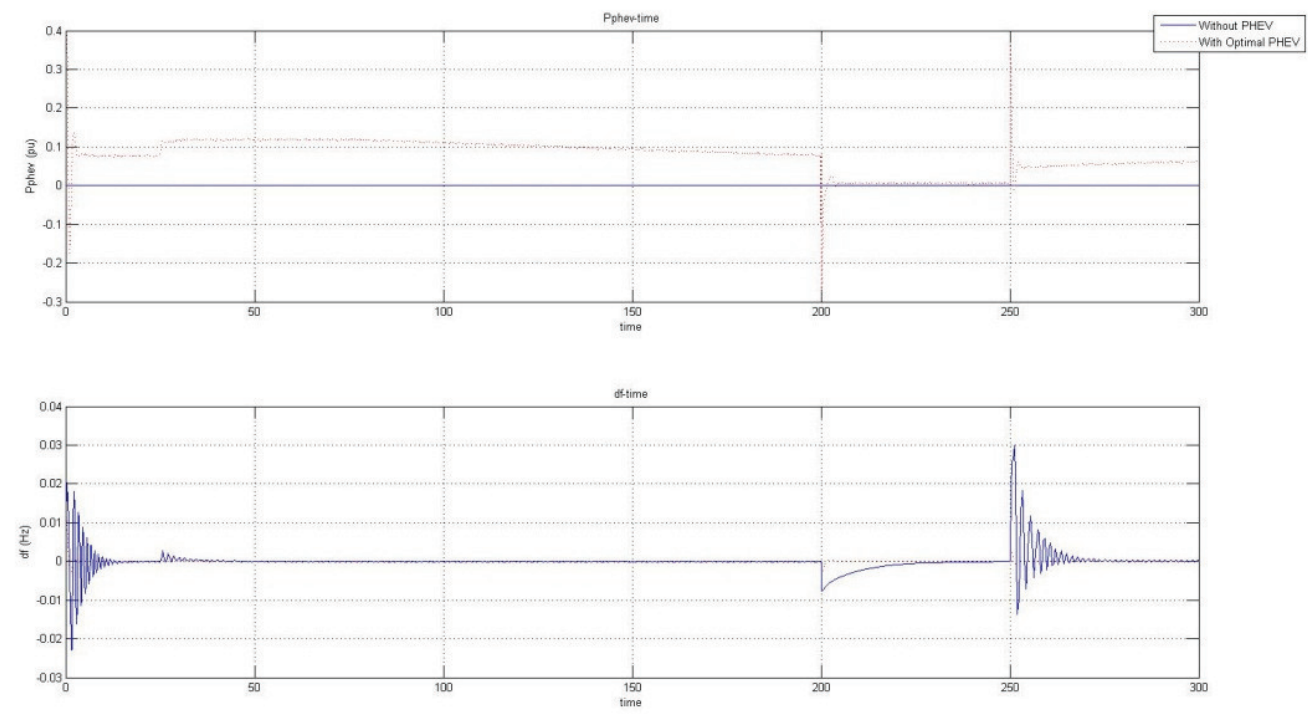

Fig. 2.36. Microgrid outputs with and without PHEV system

\section{B. Effect of controlling PHEV in microgrid frequency}

The results of using controllers for PHEVs in the system are shown in Fig. 2.37. It can be concluded that with uncontrolled PHEVs, these devices are not going to be used optimally in the microgrid system and the amount of their power output in dynamic response of the system is close to zero. However, controlled PHEV can effectively damp the frequency oscillation due to load and wind speed deviations. The frequency response shown in uncontrolled state can harmfully effect the system.
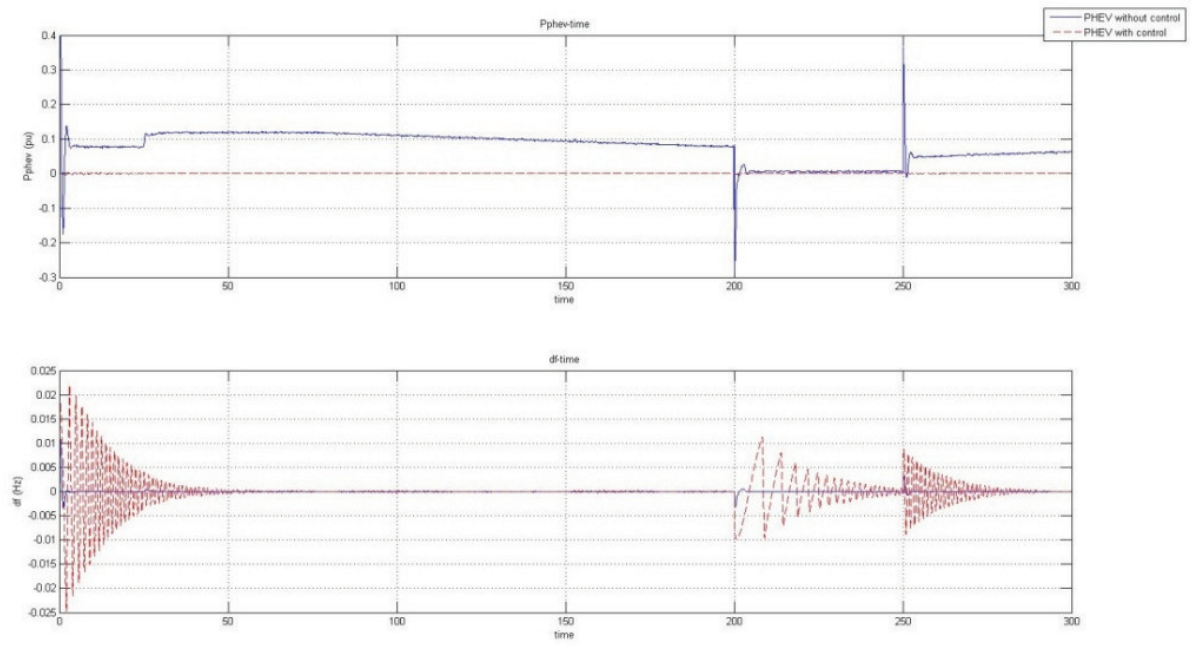

Fig. 2.37. Microgrid outputs for a controlled and uncontrolled PHEV system 
KESHTKAR: MODELING AND CONTROLLING A HYBRID MULTI-AGENT BASED MICROGRID IN PRESENCE OF DIFFERENT PHYSICAL AND CYBER COMPONENTS

C. Effect of the number of PHEVs on microgrid frequency

Different number of PHEVs are added to the system gradually and the outputs of the simulations are compared in Fig. 2.38(a). In order to magnify the results, the frequency response for the critical period of time is shown in Fig. 2.38-(b).
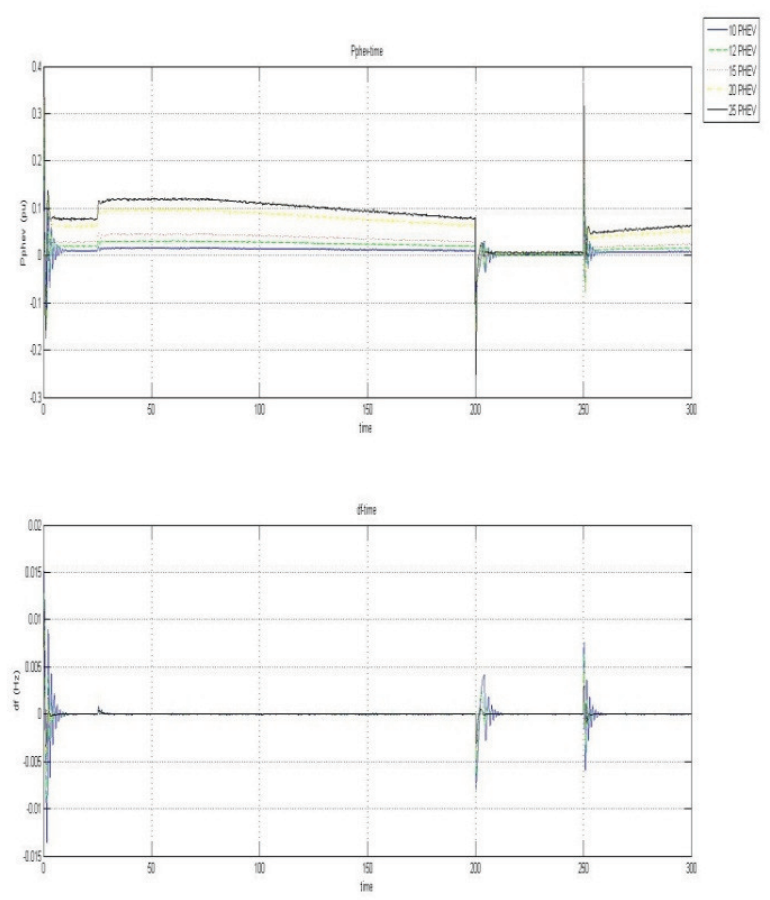

(a)

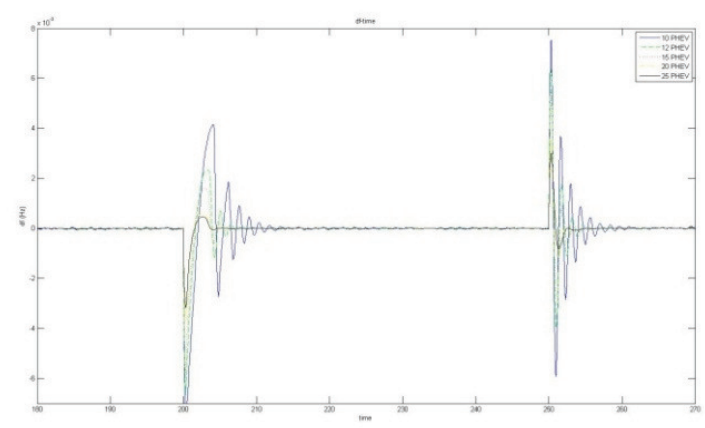

(b)

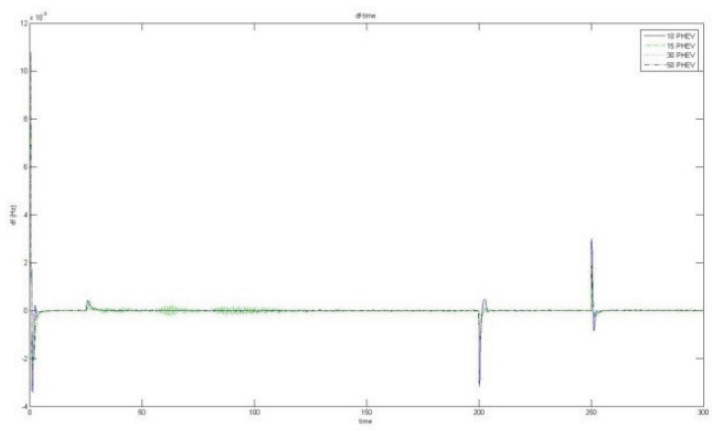

(c)

Fig. 2.38. Microgrid system outputs for different number of PHEV systems penetration. (a) PHEV power and system frequency output, (b) system frequency output, (c) frequency output in wide range of PHEV penetration 
The numbers of PHEVs shown in the results are the number of group of similar PHEVs in the system. Assume that approximately $300 \mathrm{~kW}$ contribution from PHEVs will give $1 \mathrm{~Hz}$ frequency variation. According to this approximation and knowing $\mathrm{kW}$ value of each PHEV the number of PHEVs for simulations are decided. Also, it is essential to consider that full capacity of each PHEV is not using and only 10\%-15\% capacity is used to do frequency control if individual PHEVs are charged more than $60 \%$ of their capacity.

As it can be seen, PHEVs act as fast response energy storages in the system and can inject or store power throughout the microgrid in order to damp the frequency deviation as fast as possible. Therefore, increasing the number of PHEVs in the system can improve the microgrid frequency.

\section{One of the disadvantages of using PHEV in microgrid}

The results of using different number of PHEVs in the modeled microgrid are shown in Fig. 2.38-(c). In this case large number of PHEVs is also taken into account.

It can be concluded that PHEV cannot always have positive influence on the dynamic aspects of the system. Fig. 2.38-(c) shows that with large amount of penetration of PHEVs into the system, they can act like a disturbance load for the system and can make the frequency response more oscillatory. So the amount of PHEV usage in the system should be under control.

\subsection{Stochastic PHEV}

The stochastic modelings for different PHEVs' behaviors using four stochastic processes were shown in Fig. 2.23 in part 3.4 of section II. The results of the microgrid's frequency regulation in presence of these different stochastic behaviors of PHEVs, introduced in the previous parts, are shown in Fig. 2.39.

The results for different parts are shown for a fraction of simulation time to make the comparison more suitable. The solid-line and dotted-line curves are related to without and with proposed control, respectively. Some of the stochastic process used as PHEVs behavior, shown in Fig. 2.23 in part 3.4 of section II, causes more deviation in the power outputs of these vehicles. It can be seen from Fig. 2.39 that even though stochastic behavior of PHEV makes the frequency oscillations more sever, the proposed control method is damping the deviations appropriately in transient and steady state point of view.
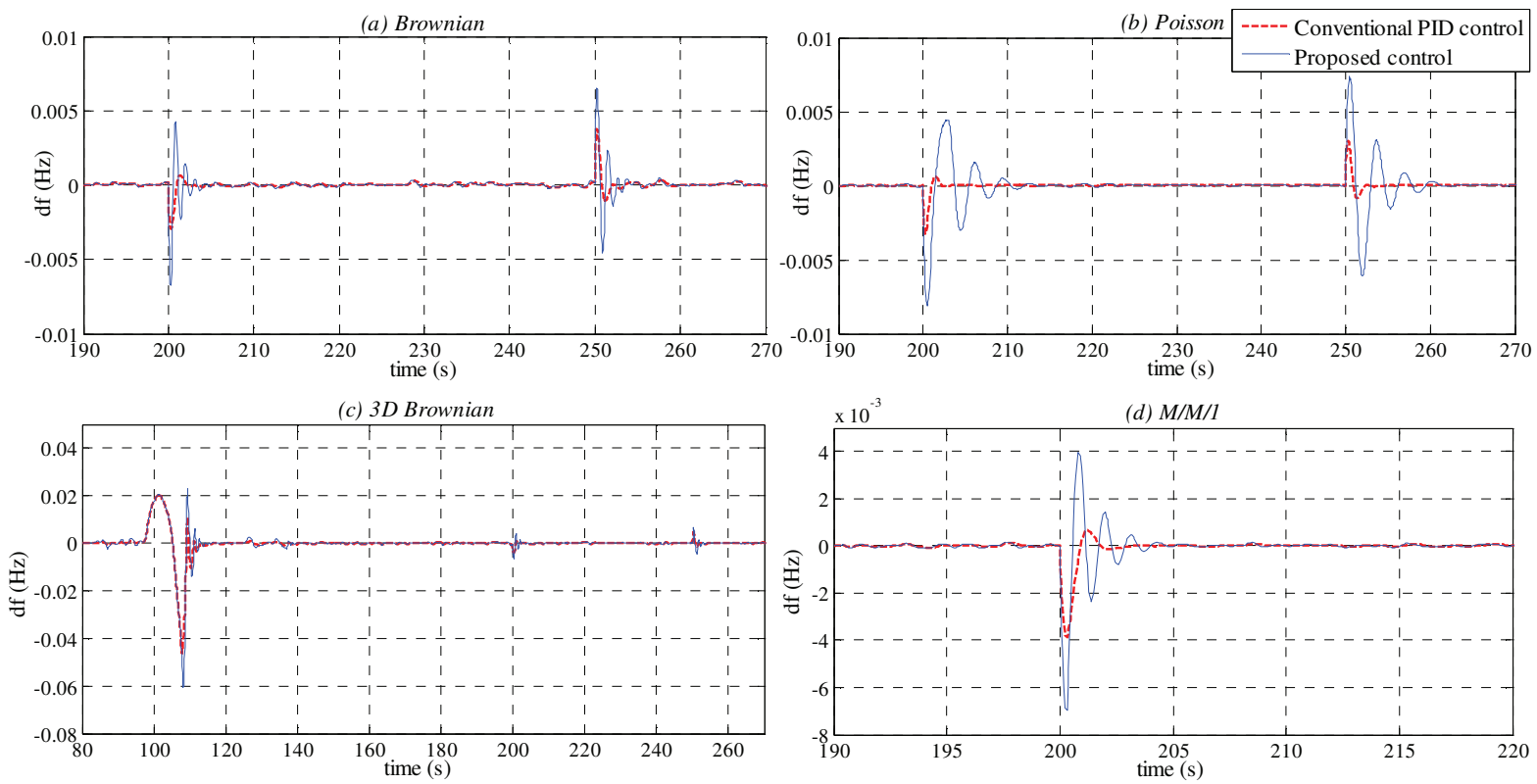

Fig. 2.39. Frequency deviation results with conventional and proposed control method; (a) brownian, (b) poission, (c) 3d Brownian, (d) M/M/1 


\section{KESHTKAR: MODELING AND CONTROLLING A HYBRID MULTI-AGENT BASED MICROGRID IN PRESENCE} OF DIFFERENT PHYSICAL AND CYBER COMPONENTS

\section{MULTI AGENT SIMULATIONS}

\subsection{Frequency oscillations due to power mismatch}

In order to evaluate the proposed control method and make a comparison between the proposed optimal LQR controller in part 4.1 section II and conventional PI controller, first part of this part is allocated to this analysis. The next part studies the effectiveness of the proposed control methods when the modeled microgrid faces cyber attacks. Implementation of the proposed detection and learning algorithm simulated in presence of cyber attacks. Three different control methods are evaluated and compared in the last part of the simulations.

In this part, considering different cases, two control algorithms are analyzed when the microgrid face frequency deviations due to sudden power mismatch between generation and demand.

\section{Case 1: Increasing in generating power}

In this case the output of wind and solar has been increased by $0.5 \%$ of initial value. Fig. 2.40 depicts the change in generating power and shows how different control approaches damp the frequency oscillation. The blue curve shows the result related to PI control, and the red one shows the result related to LQR control

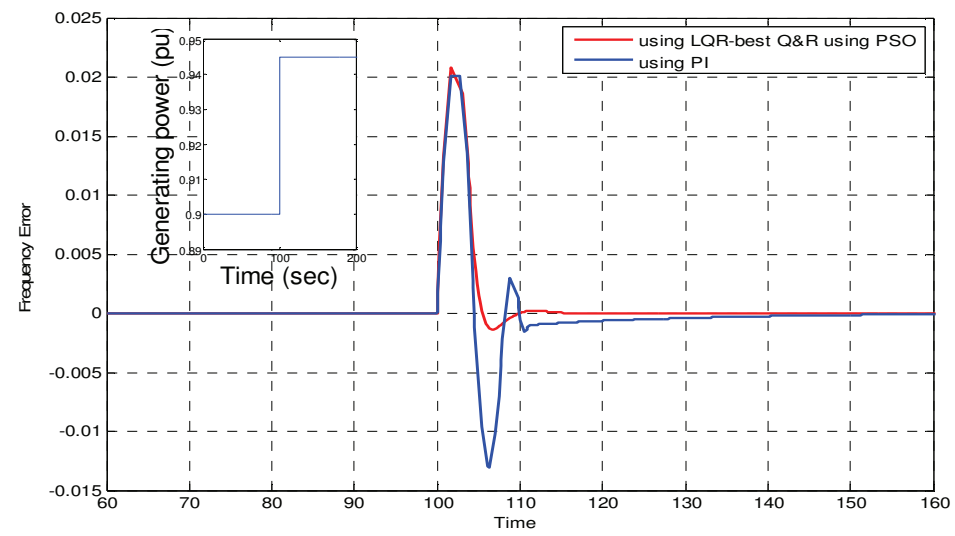

Fig. 2.40. Frequency response after increasing the generating power

In this case because the generating power is more than the demand power, the diesel generator and fuel cell do not generate power so just the aqua electrolyzer is responsible to damp the frequency oscillation. As it can be seen frequency suddenly rises due to the extra generating power comparing to demand and the controllers bring it back to nominal value. Comparison shows that the proposed control method controls the frequency response of the microgrid much better than the conventional method. Fig. 2.41 shows the controller input for aqua electrolyzer in different scheme.

It can be seen that proposed LQR control method minimizes the participation of aqua electrolyzer in damping the frequency deviation in the system. 


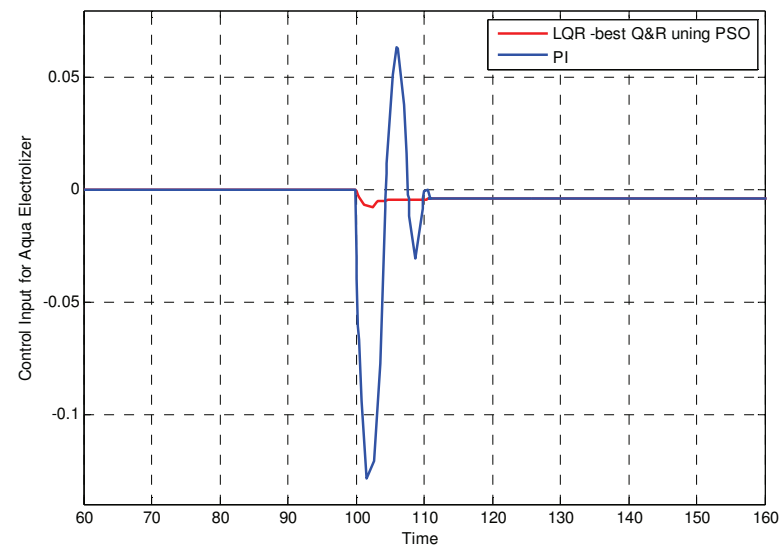

Fig. 2.41. Control input of $\mathrm{AE}$ after increasing the generating power

\section{Case 2: Decrease in generating power}

In this case the output of wind and solar has been decreased by $0.5 \%$ of initial value. Fig. 2.42 depicts the change in generating power and shows how different control approaches damp the frequency oscillation. The blue curve shows the result related to PI control and the red one shows the result related to LQR control.

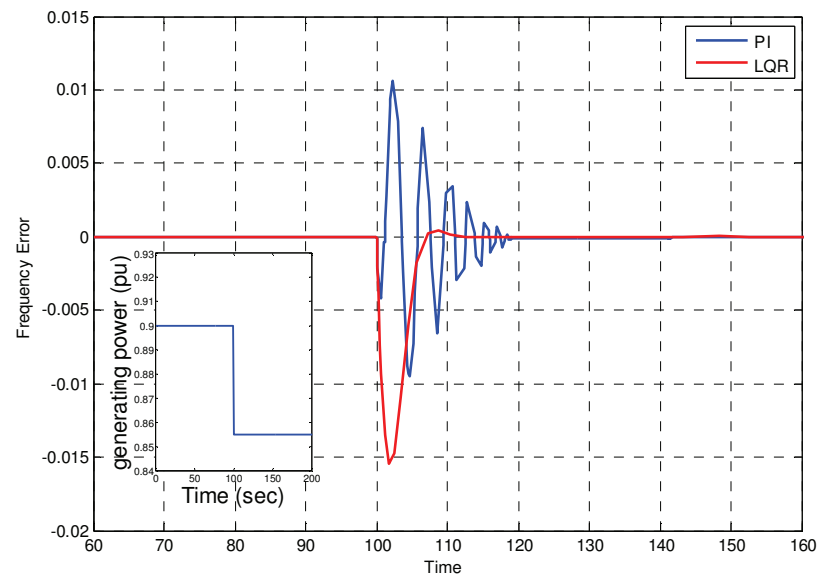

Fig. 2.42. Frequency response after decreasing the generating power

It can be seen that due to extra demand after the decrease in generating power, the frequency drops and then is brought back to nominal value by the controllers. Fig. 2.42 shows the effectiveness of the proposed control algorithm comparing to conventional method. In this case because the generating power is less than the demand power, the diesel generator and fuel cell should generate power to adjust the frequency error and the aqua electrolyzer is not participating in damping the frequency oscillation. Fig. 2.43 and 2.44 show the controller input for the diesel generator and fuel cell with LQR algorithm.

These figures show how these Distributed Generators (DGs) contributed in frequency regulation of the microgrid after a disturbance happens in the system. 


\section{KESHTKAR: MODELING AND CONTROLLING A HYBRID MULTI-AGENT BASED MICROGRID IN PRESENCE OF DIFFERENT PHYSICAL AND CYBER COMPONENTS}

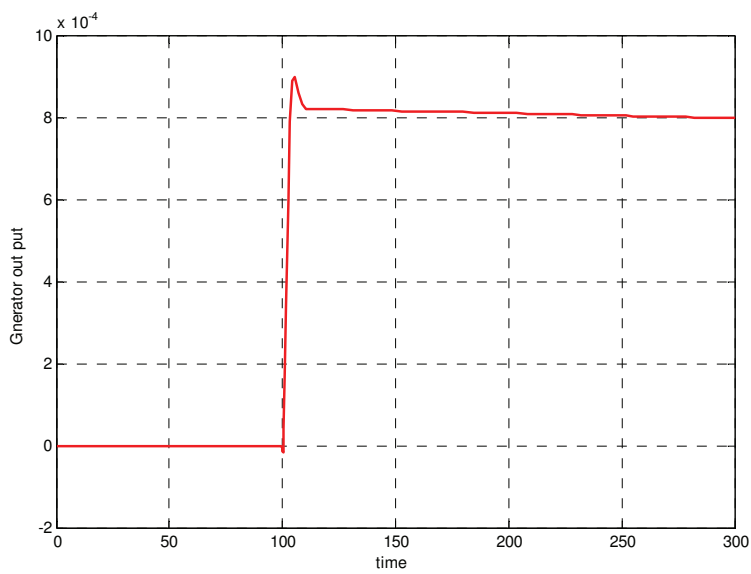

Fig. 2.43. Controller input for the diesel generator

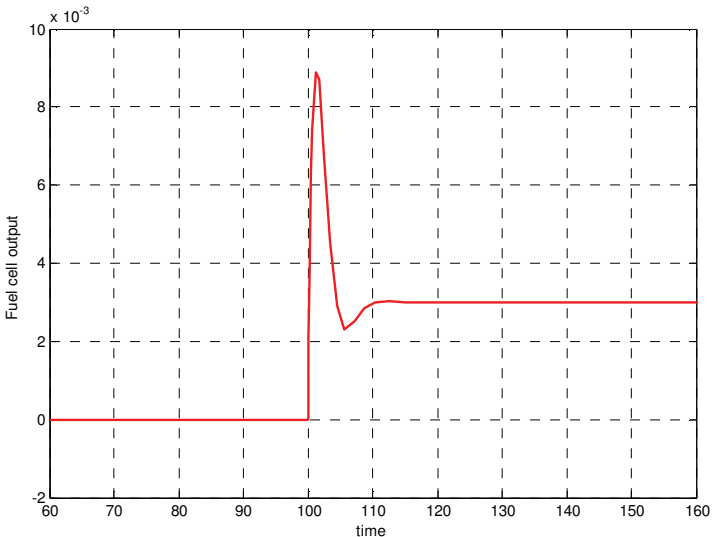

Fig. 2.44. Controller input for the fuel cell

\subsection{Frequency oscillations due to cyber attacks}

In this part, the robustness of the proposed controllers has been examined in presence of different kinds of cyber attacks on the frequency measurement signals with different characteristics. Firstly, DHP control approach which is explained in part 4.1 of section II along with the proposed defense strategy, is employed for attack mitigation and frequency control purpose in presence of different intrusions. Then, the performance of DHP is compared with LQR and conventional PI control methods.

\section{Case 1: Moderate Replay attack}

Replay attacks are cyber attacks which break the integrity or more precisely the freshness of measurements data. In other words, we consider defense against replay attack where an adversary records a sequence of sensor measurements and replays the sequence afterwards. This kind of attack has been simulated in the studied microgrid. It is implemented on the frequency signal which goes toward the controllers in the MGCC of the modeled microgrid.

Fig. 2.45.a shows the frequency response of the system having the proposed controller due to a sudden load rise in the power microgrid. It can be seen that the control algorithm reacts appropriately to the frequency perturbation of the system and damped this disturbance in the system. 
A moderate replay attack is modeled on the frequency signal which transmits to Microgrid Central Control (MGCC) to be used as input for the controller. The resulted frequency signal after facing cyber attack is shown in Fig. 2.45.b It can be seen that the frequency signal between 100s and 120s has been retransmitted in 500s (red dashed curve), when the system is supposed to be stable and in steady state.

It can be seen from blue solid curve that the proposed algorithm quickly act against the frequency oscillations due to the replay attack at 500 s and damped the deviations in proper time.
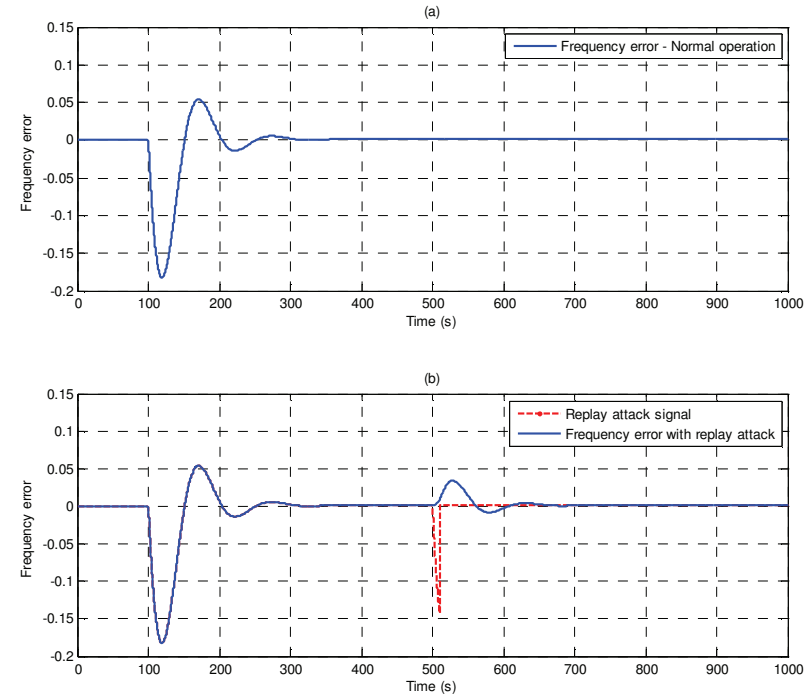

Fig. 2.45. Frequency response of the system; (a) without attack; (b) after moderate replay attack

\section{Case 2: Sever Replay attack}

In this case, as it is shown in Fig. 2.46.a, the same replay attack happens at time $150 \mathrm{~s}$, when the frequency is still in transient time after the sudden load change (red dashed curve). This case is more severe because the frequency deviation signal at this time is supposed to be increased positively but due to the replay attack it is transmitted as a negatively increasing signal. This causes the controller act wrong at the very first moment and worsens the frequency oscillations. However, the controller shows robustness against this attack and damps the frequency deviations appropriately.
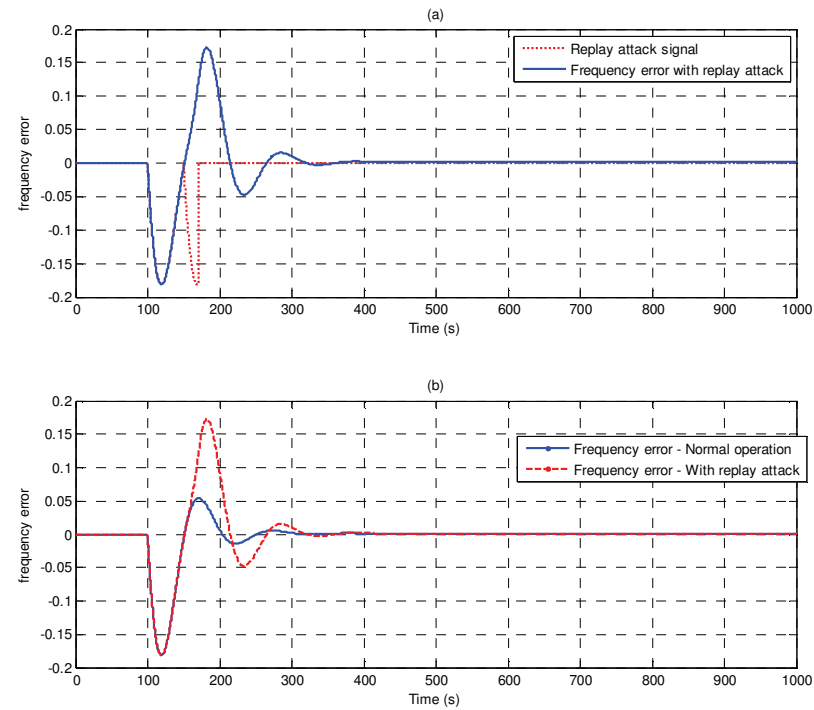

Fig. 2.46. Frequency response of the system; (a) after severe replay attack; (b) with and without severe replay attack

Fig. 2.46.b shows the frequency response of the microgrid with and without severe replay attack. 
KESHTKAR: MODELING AND CONTROLLING A HYBRID MULTI-AGENT BASED MICROGRID IN PRESENCE OF DIFFERENT PHYSICAL AND CYBER COMPONENTS

It can be seen that the time of the replay attack really affects the severness of the consequence in the frequency response of the system. Additionally, the proposed control method shows appropriate robustness against this kind of attack with different time of happening.

\section{Case 3: Short time Data loss}

Second kind of attack which has been modeled in this work is data loss. Generally, the packet loss condition may occur in UDP/IP system. So, the effect of information loss has been studied in this case. It is assumed that the attack has happened on the frequency signal which is the input of the controller and play an important role in the stability of the system. As it can be seen form Fig. 2.47.a, the data loss has happened at $120 \mathrm{~s}$. MGCC is designed to use the last value for the period of time that the data has been lost. The red dashed curve in Fig. 2.47.a shows the period of attack time and the value of frequency signal which has been used during the attack period. Fig. 2.47.b shows the frequency response with and without happening of data loss in the frequency response of the system.
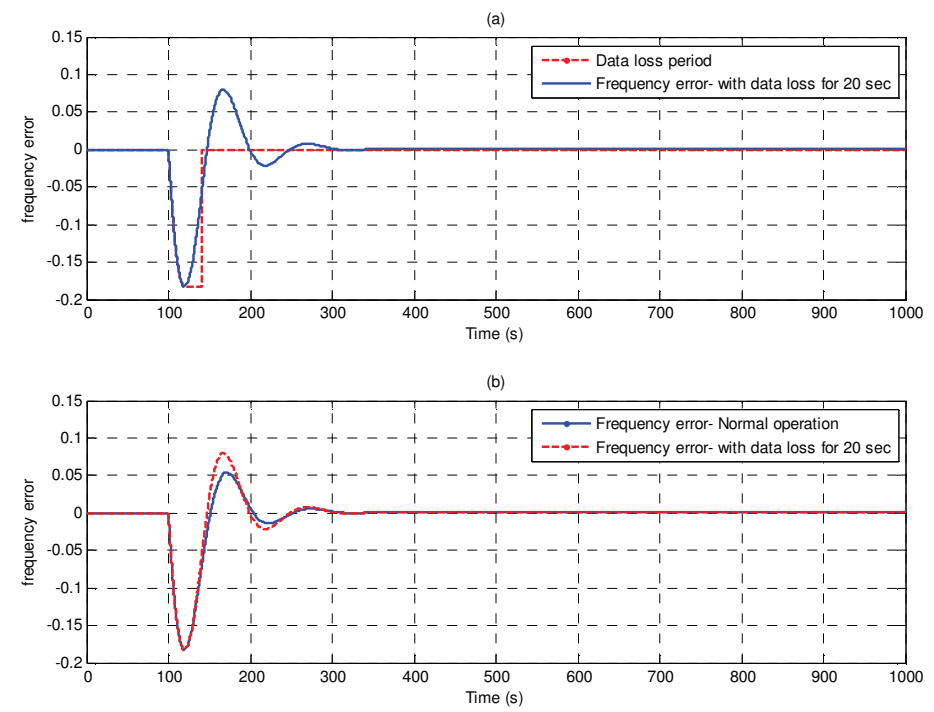

Fig. 2.47. (a) Frequency response and attack model with short time data loss; (b) Frequency response with and without short time data loss

It can be seen from Fig. 2.47.b that data loss can increase the intensity of the frequency oscillations in the power system (red dashed curve) and how the proposed control algorithm can handle this perturbation in the system.

\section{Case 4: Longer time Data loss}

In this case, the duration of cyber attack has been increased and data loss of the frequency signal lasts longer than previous case. As it can be seen from the red dashed curve in Fig. 2.48.a, the data loss lasts 50 seconds which can be very critical for the transient responses of the system.

Additionally, the difference between the real value of frequency signal and the transmitted signal is a lot more than the previous case. Fig. 2.48.b shows the performance of controller with/without this data loss attack.

It can be seen from Fig. 2.48.b that frequency deviates a lot from the nominal value and make it in a potential situation of instability. This is because of the longer duration of data loss and the difference between the actual signal and transmitted one at the time of attack. Furthermore, the effectiveness of the proposed control approach can be concluded from Fig. 2.48.b. 

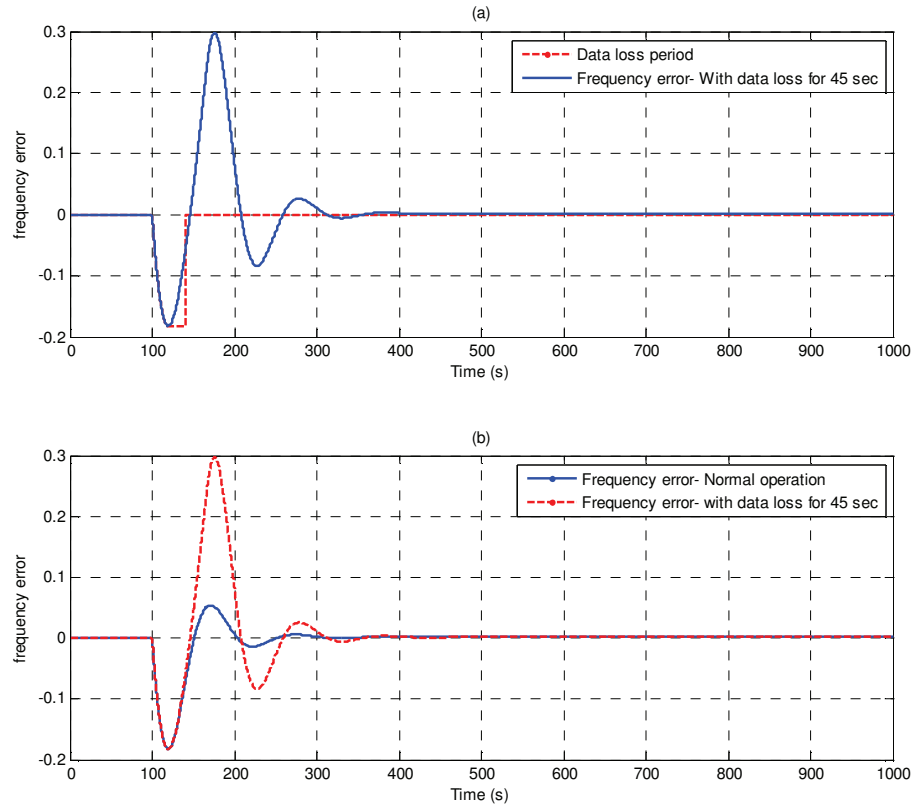

Fig. 2.48. (a) Frequency and attack model of the system with longer time data loss; (b) Frequency response with and without longer time data loss

\subsection{Implementation of detection and learning methods}

In this case, detection method is used to recognize the happening of an attack and inform the controllers of the starting and ending time of the attacks. The frequency signal in the period of a replay attack (dashed red curve) and the detection signal (solid blue curve) are shown in Fig. 2.49.a. As it can be seen, the proposed detection method is able to recognize the starting and ending time of the attack and gives alarm signal at time 150s and 200s. It should be noticed that the signal at time 100s (which is due to a normal load change) is not higher than pre-defined threshold to be detected.
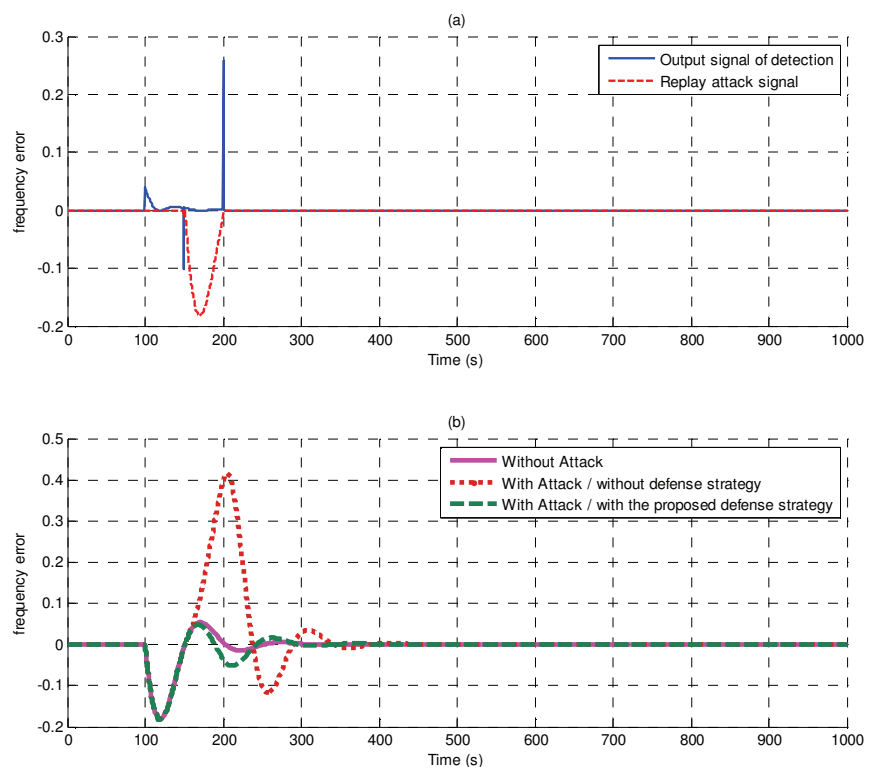

Fig. 2.49. (a) Detection algorithm output in case of a replay cyber attack; (b) Frequency response with/without detection and learning (defense) algorithm

As soon as receiving the attack alarm, The MGCC starts using the frequency signal providing by learning algorithm. Therefore, the controller parameters are immediately set based on the value obtained from training in this particular frequency oscillation. As it can be seen from Fig. 2.49.b, the frequency response of the system improved considerably comparing to the case without the proposed detection and learning algorithms. 
KESHTKAR: MODELING AND CONTROLLING A HYBRID MULTI-AGENT BASED MICROGRID IN PRESENCE OF DIFFERENT PHYSICAL AND CYBER COMPONENTS

\subsection{Comparing the control methods}

In this case, the application of conventional and proposed methods which are described in in part 4.1 of section II are compared in the case of a replay cyber attack which is modeled in this part. The attack occurs on the frequency measurement signal in the modeled multi-agent microgrid. Then the detection and learning algorithm as the proposed defense strategy along with three different control approaches including PI, LQR, and DHP are used in the MGCC of the system to control the disturbance caused by the attack in the frequency response of the system. The optimized parameters of PI method are shown in Table 2.VIII.

Table 2.VIII. PI Parameters of the studied system

\begin{tabular}{|c|c|c|c|c|c|}
\hline \multicolumn{2}{|c|}{ DEG } & \multicolumn{2}{c|}{ FC/AE } & \multicolumn{2}{c|}{ SMES } \\
\hline$P_{\text {deg }}$ & $I_{\text {deg }}$ & $P_{\text {fc/ae }}$ & $I_{f_{c} / a e}$ & $P_{\text {smes }}$ & $I_{\text {smes }}$ \\
\hline 492.3 & 227.3 & 312.1 & 101.9 & 487.6 & 499.7 \\
\hline
\end{tabular}

It is concluded that DHP method as a fast acting approach is able to damp the frequency deviation more appropriate than the other two methods. In addition, it can be concluded that conventional PI controller is the worst option to be selected in these case studies comparing to DHP and LQR, and can cause instability against more sever attack situations.

\section{IV- CONClusions OF CHAPTER 2}

In the first part of this chapter, this dissertation studies the LFC problem in a microgrid power system and the effect of renewable energy sources, DG, and ESS, as well as the effect PHEV penetration on microgrid stability. The objective function used in the control method is the deviation of frequency which may appear or increase due to disturbances imposed to the microgrid. Simulation results exhibit efficient and robust performance of the proposed methods to protect the system against possible instabilities related to load and wind power variations in microgrid. ESSs, especially fast-acting ones can participate in improving the stability and transient specification of microgrid frequency.

Then, a multi-objective optimization based on differential evolutionary has been applied to the speed governor of a microturbine system to find the best controller parameters in order to have the most suitable specification for both speed deviation and fuel consumption, simultaneously after load changing during the daily demand. The results showed that the parameters related to the same-weighting factor optimization are definitely better than those which obtained in speed or fuel weighted factor optimization. Therefore, the most suitable speed governor system has been designed, and the least variation of speed and total fuel consumption against daily load changing has been obtained. The proposed model is added to a Microgrid in order to be participated in frequency control problem. The same load change for the MT modeling has been considered as disturbance in this problem. The results show that this modeled Microgrid has the best transient response due to fast-acting MT participation as well as the most economic design due to its minimum fuel consumption.

In the next part, based on the results of PHEV modeling, in dynamic point of view, it is seen that they can have both advantages and disadvantages for the system which they are using for. As an advantage, PHEVs can perform as energy storage for the system that their output can be useful in the case of deviation in system response. However, uncontrolled use of PHEVs can act as a load disturbance for the system and make the outputs more oscillatory. Additionally, effect of the amount of PHEV penetration and stochastic behavior of them to the system is also studied by increasing the number of stochastic PHEVs in the microgrid. It can be concluded that existing reasonable number of controlled PHEVs in microgrid cane make us able to take advantage of its good 
effect on frequency response of the system and to avoid its inappropriate characteristics for dynamic of the distribution power system.

In the last part of this chapter, a study has been done in order to analyze the application of optimal state feedback LQR control and ADP (DHP) which is proposed in this chapter for a smart microgrid. The optimization algorithm which is used for designing the $\mathrm{Q}$ and $\mathrm{R}$ in $\mathrm{LQR}$ and also PI control parameters to minimize the frequency oscillations is Particle Swarm Optimization (PSO) which is fast enough for transient analysis. This algorithm has been discussed in a separate section. The modeling of the microgrid and the central control of it has been discussed in this chapter. A comparison has been made between conventional PI controller and the proposed optimal state feedback LQR and ADP control performance in the simulation results section. The effectiveness of the proposed LQR and ADP control has been concluded from the observations. In this part the robustness of the proposed controller has been tested against different cyber attacks with different timing and severity. Simulation results show that the proposed method is fast enough and act appropriately and accordingly when facing different kinds of cyber attacks on the critical signals of the system. A detection and learning algorithm is proposed as a defense strategy against cyber attacks for improving the resiliency of the system in case of these attacks. When a cyber attack occurs on the frequency measurement signal of the multiagent based microgrid, this detection and defense strategy along with the control approaches are used in the MGCC to damp the frequency oscillations. The results show the effectiveness of the proposed algorithm in case of cyber intrusions.

\section{V- REFERENCES FOR CHAPTER 2}

[1] S. Mishra, G. Mallesham, and P. C. Sekhar, "Biogeography Based Optimal State Feedback Controller for Frequency Regulation of a Smart Microgrid ", IEEE Transaction on Smart Grid, vol. 4, no. 1, March 2013.

[2] I. J. Balaguer, Q. Lei, S. Yang, U. Supatti, and F. Z. Peng, "Control for grid-connected and intentional islanding operations of distributed power generation," IEEE Trans. Ind. Electron., vol. 58, no. 1, Jan. 2011.

[3] R.Majumder, B. Chaudhuri, A. Ghosh, R.Majumder, G. Ledwich, and F. Zare, "Improvement of stability and load sharing in an autonomous microgrid using supplementary droop control loop," IEEE Trans. Power Syst., vol. 25, no. 2, pp. 796-808, May 2010.

[4] F. Valenciaga, P. F. Puleston, and P. E. Battaiotto, "Power control of a solar/wind generation system without wind measurement: A passivity/ sliding mode approach,"IEEE Trans. Energy Convers., vol. 18, no. 4.

[5] S. Bhowmik, K. Tomsovic, and A. Bose, "Communication models for third party load frequency control," IEEE Trans. Power Syst., vol. 19, no. 1, pp. 543548, Feb. 2004.

[6] T. S. Bhatti and D. P. Kothari, "Variable structure load-frequency control of isolated wind-diesel-microhydro hybrid power systems," J. Inst. Eng., vol. 83, pp. 52-56, 2002.

[7] B. Kroposki, T. Basso, and R. DeBlasio,” Microgrid Standards and Technologies”, Power and Energy Society General Meeting, IEEE, pp. 1-4, July 2008 .

[8] A. A. El-Emary and M. A. El-Shibina, “Application of static VAR compensation for load frequency control,” Elect. Machines Power Syst., vol. 25, no. 9, pp. 1009-1022, Nov. 1997.

[9] H. Asano, K. Yajima, and Y. Kaya, "Influence of photovoltaic power generation on required capacity for load frequency control," IEEE Trans. Energy Convers., vol. 11, no. 1, pp. 188-193, Mar. 1996.

[10] S. Bhowmik, K. Tomsovic, and A. Bose, "Communication models for third party load frequency control," IEEE Trans. Power Syst., vol. 19, no. 1, pp. 543548, Feb. 2004.

[11] T. S. Bhatti and D. P. Kothari, "Variable structure load-frequency control of isolated wind-diesel-microhydro hybrid power systems," J. Inst. Eng., vol. 83, pp. 52-56, 2002

[12] S. Y.M. Atwa, E.F. El-Saadany, M.M.A. Salama, R. Seethapathy, " Optimal Renewable Resources Mix for Distribution System Energy Loss Minimization", IEEE Trans on Power Systems, vol. 25, no. 1, 2010.

[13] S. Mishra, G. Mallesham, and P. C. Sekhar, "Biogeography Based Optimal State Feedback Controller for Frequency Regulation of a Smart Microgrid ", IEEE Transaction on Smart Grid, vol. 4, no. 1, March 2013.

[14] I. J. Balaguer, Q. Lei, S. Yang, U. Supatti, and F. Z. Peng, "Control for grid-connected and intentional islanding operations of distributed power generation," IEEE Trans. Ind. Electron., vol. 58, no. 1, Jan. 2011.

[15] M. A. Hassan, M. A. Abido, “Optimal design of microgrids in autonomous and grid-connected modes using PSO,”IEEE Trans. Power Electron., vol. 26, no. 3, pp. 755-769, Mar. 2011.

[16] S. Kazemlou, S. Mehraeen,, "Stability of multi-generator power system with penetration of renewable energy sources", Power and Energy Society General Meeting, IEEE 2012.

[17] S. Y.M.Atwa, E.F. El-Saadany, M.M.A.Salama, R.Seethapathy, "Optimal Renewable Resources Mix for Distribution System Energy Loss Minimization", IEEE Trans on Power Systems, vol. 25, no. 1, 2010. 
KESHTKAR: MODELING AND CONTROLLING A HYBRID MULTI-AGENT BASED MICROGRID IN PRESENCE OF DIFFERENT PHYSICAL AND CYBER COMPONENTS

[18] Dong-Jing Lee; Li Wang, "Small-Signal Stability Analysis of an Autonomous Hybrid Renewable Energy Power Generation/Energy Storage System Part I: Time-Domain Simulations," in IEEE Transactions on Energy Conversion, , vol.23, no.1, pp.311-320, March 2008.

[19] Das Rajesh Joseph Abraham D, AmitPatra. AGC of a hydrothermal system with thyristor controlled phase shifter in the tie-line. In: IEEE power India conference; 2006.

[20] Tripathy SC, Balasubramanian R, Chandramohanan Nair PS. Effect of superconducting magnetic energy storage on automatic generation control considering governor deadband and boiler dynamics. IEEE Trans Power Syst 1992;7(3):1266-73.

[21] Banerjee S, Chatterjee JK, Tripathy. Application of magnetic energy storage unit as load frequency stabilizer. IEEE Trans Energy Conv 1990;5(1):46-51.

[22] H. Keshtkar, J. Solanki, S. K. Solanki, "Dynamic modeling, control and stability analysis of microturbine in a microgrid," in T\&D Conference and Exposition, 2014 IEEE PES, vol., no., pp.1-9, 14-17 April 2014.

[23] F. Doostmohammadi, A. Esmaili, S.H.H. Sadeghi, A. Nasiri, H.A. Talebi, "A model predictive method for efficient power ramp rate control of wind turbines", 38th Annual Conference on IEEE Industrial Electronics Society IECON, pp. 881 -885, IEEE 2012.

[24] Sitthidet Vachirasricirikul, Issarachai Ngamroo, "Robust controller design of microturbine and electrolyzer for frequency stabilization in a microgrid system with plug-in hybrid electric vehicles”, Elsevier Journal, Electrical Power and Energy Systems 43 (2012) 804-811.

[25] A.K.Saha, S.P.Chowdhury, S.Chowdhury, P.A.Crossley and C.T.Gaunt, "Microturbine Based Distributed Generatorin Smart Grid Application", 20th International Conference on Electricity Distribution, pp.1052-1057, June 2009.

[26] Y. Lee, Y. Chang, C. Chan, Y. Ho, "Preliminary Implementation of Microgrid with Photovoltaic and Microturbine for Stand Alone Operation" IEEE Industry Applications Society Annual Meeting (IAS), 2012, pp. 1-9.

[27] Sreedhar R.Guda, C.Wang, M.H.Nehrir, “A Simulink-Based Microturbine Model for Distributed Generation Studies”, IEEE, pp.269-274, 2005.

[28] F. C. Lee, P. Barbosa, X. Peng, J. Zhang, B. Yang and F. Canales, "Topologies and design considerations for distributed power system applications", Proceedings of the IEEE, Vol. 23, No. 3, pp.1383 - 1392, Aug 2008.

[29] C. Lin and S. Lin, "Distributed Optimal Power Flow With DiscreteControl Variables of Large Distributed Power Systems", IEEE Transactions on Power Systems, Vol. 23, No. 3, pp.1383 - 1392, Aug.2008.

[30] S. Luo, "A review of distributed power systems part I: DC distributed power system”, IEEE Aerospace and Electronic Systems Magazine, Vol. 20, No. 8, Part 2, pp. 5 - 16, Aug. 2005.

[31] Sitthidet Vachirasricirikula, Issarachai Ngamroob, Somyot Kaitwanidvilaib, " Application of electrolyzer system to enhance frequency stabilization effect of microturbine in a microgrid system" International Journal of hydrogen energy (2010), pp. 7131-7142.

[32] He Wang, and Guoqing Li, ” Dynamic Performance of Microturbine and Fuel Cell in a Microgrid” International Conference on Mechatronic Science, Electric Engineering and Computer, August 19-22, 2011, pp. 122-125.

[33] C. J. Hatziadoniu, A. A. Lobo, F. Pourboghrat and M. Daneshdoost, “A simplified dynamic model of grid-connected fuel-cell generators”, IEEE Transactions on Power Delivery, Vol.17, No. 2, pp. 467.473, Apr. 2002.

[34] C. Hatziadoniu, A. Lobo, F. Pourboghrat and M. Daneshdoost, “A Simplified Dynamic Model of Grid-Connected Fuel-Cell Generators”, IEEE Power Engineering Review, Vol.21, No.11, pp. 62-62, Nov. 2001.

[35] F. Pai and S. Huang, "Design and Operation of Power Converter for Microturbine Powered Distributed Generator with Capacity Expansion Capability", IEEE Transaction on Energy Conversion, Vol. 23, No. 1, pp.110 - 118, March 2008 .

[36] K. Sedghisigarchi and A. Feliachi, "Dynamic and transient analysis of power distribution systems with fuel Cells-part II: control and stability enhancement", IEEE Transaction on Energy Conversion, Vol.19, No. 2, pp. 429 - 434, June 2004.

[37] M.D. Galus, S. Koch, and G. Andersson, "Provision of Load Frequency Control by PHEVs, Controllable Loads, and a Cogeneration Unit", IEEE Transactions on Industrial Electronics, Vol. 58, No. 10, October 2011, pp. 4567-4582.

[38] Takagi, M. ; Yamaji, K. ; Yamamoto, H., "Power system stabilization by charging power management of Plug-in Hybrid Electric Vehicles with LFC signal," IEEE Vehicle Power and Propulsion Conference, 2009. VPPC '09. pp. 822 - 826.

[39] O. Hegazy, , J. Van Mierlo, P.Lataire and M. El Baghdadi, "Integrated Power Electronics Interface for Plug-In Hybrid Electric Vehicle Applications",CIPS 2012, March, 6-8, 2012, Nuremberg/Germany.

[40] Michael Kintner-Meyer, Kevin Schneider and Robert Pratt, "Impacts Assessment of Plug-In Hybrid Vehicles on Electric Utilities and Regional U.S. Power Grids,” Technical Report Pacific Northwest National Laboratory, Atlanta GA, 17-18 November, 2008.

[41] Denholm, P., and W. Short, “An Evaluation of Utility System Impacts and Benefits of Optimally Dispatched Plug-In Hybrid Electric Vehicles," Tehcnical Report NREL/TP-620-40293, Golden CO, 2006.

[42] Curtis Roe and Jerome Meisel, "Power system level impacts of plug-in hybrid electric vehicles using simulation data," IEEE Energy2030, Atlanta GA, 1718 November, 2008. 
[43] K. Parks, P. Denholm, and T. Markel, “Charging in the Xcel Energy Colorado Service Territory,” Technical Report NREL/TP-640-41410, Golden CO, 811 May, 2007.

[44] The Smart Grid: An Introduction. DOE Smart Grid Book, 2008.

[45] S. Wirasingha, N. Schofield, and A. Emadi, "Plug-in hybrid electric vehicle developments in the us: Trends, barriers, and economic feasibility," in Vehicle Power and Propulsion Conference. IEEE, 2008, pp. 1-8.

[46] Ebrahim, M.A. ; Mostafa, H. E. ; Gawish, S.A. ; Bendary, F.M. "Design of Decentralized Load Frequency Based-PID Controller Using Stochastic Particle Swarm Optimization Technique ", International ConferenceonElectric Power and Energy Conversion Systems,EPECS '09, pp.1-6.

[47] Mostafa, O.M, "Handling dynamic uncertainties for electric power system modeling and control ", IEEE Conference on Decision and Control including the 15th Symposium on Adaptive Processes, 1976, pp. 267-271.

[48] Liang, J. ; Molina, D. D. ; Venayagamoorthy, G. K. ; Harley, R. G, "Two-Level Dynamic Stochastic Optimal Power Flow Control for Power Systems With Intermittent Renewable Generation", IEEE Transactions on Power System, Issues. 9. pp. 1-9, 2013.

[49] F. Cohen, "Simulating Cyber Attacks, Defenses, and Consequences" Computers \& Security, 18 (1999), pp. 479-518,1999.

[50] W. Wang, Z. Lu, “Cyber security in the Smart Grid: Survey and challenges” Computer Networks, 57, pp. 1344-1371, 2013.

[51] M. Burmester, E. Magkos, V. Chrissikopoulos, "Modeling security in cyber-physical systems",International Journal of Critical Infrastructure Protection, pp. 118-126, 2012.

[52] E. Yuksel, H. Zhu, H. Nielson, H. Huang, and F. Nielson, "Modeling and Analysis of Smart Grid A Stochastic Model Checking Case Study",IEEE Sixth International Symposium on Theoretical Aspects of Software Engineering, pp.25-32, 2012.

[53] H.Yi , Li Husheng, K.A.Campbell, Han Zhu , "Defending false data injection attack on smart grid network using adaptive CUSUM test", Conference on Information Sciences and Systems (CISS), IEEE 2011.

[54] C. Ten, G. Manimaran, C. Liu, "Cyber security for Critical Infrastructures: Attack and Defense Modeling", IEEE Transactions on Systems, Man and Cybernetics, Part A: Systems and Humans, vol. 40, no. 4, pp. 853 - 865, July 2010.

[55] J. Kim, L. Tong, "On topology attack of a smart grid”, PES Innovative Smart Grid Technologies (ISGT), IEEE 2013.

[56] M H. Khurana, "Moving beyond defense-in-depth to strategic resilience for critical control systems", PES General Meeting, pp. 1-3, IEEE 2011.

[57] S.Han, S. Han, and K. Sezaki, "Development of an Optimal Vehicle-to-Grid Aggregator for Frequency Regulation ", IEEE Transactions on Smart Grid, Vol. 1, No. 1, June 2010, pp.65-72.

[58] M. A. Hassan, M. A. Abido, "Optimal design of microgrids in autonomous and grid-connected modes using PSO,"IEEE Trans. Power Electron., vol. 26, no. 3, pp. 755-769, Mar. 2011.

[59] S.R. Guda, C. Wang, M.H. Nehrir, "A Simulink-based microturbine model for distributed generation studies," in Power Symposium, 2005. Proceedings of the 37th Annual North American, vol., no., pp.269-274, 23-25 Oct. 2005.

[60] Reliability Test System Task Force, “The IEEE reliability test system 1996,” IEEE Trans. Power Syst., vol. 14, no. 3, pp. 1010-1020, Aug.1999.

[61] Elbeltagi, T. Hegazy, and D. Grierson, "Comparison among five evolutionary-based optimization algorithms, " Advanced Engineering Informatics, vol. 19, no. 1, pp. 43-53, 2005.

[62] A. Darvishi, A. Alimardani, B. Vahidi, S. H. Hosseinian, "Bacterial Foraging-based Algorithm Optimization based on Fuzzy Multi-objective Technique for Optimal Power Flow Dispatch", Science International, vol. 26, pp.1057-1064, 2014.

[63] W. Gu, C. Shen, Z. Wu, "Multi-Agent Based Frequency Control of Islanded Microgrid," International Review oj Electrical Engineering, vol. 6, no. 7, pp. 3134-3141, Nov. 2011.

[64] Y. Xu, and W. Liu, "Novel Multi agent Based Load Restoration Algorithm for Microgrids," IEEE Trans. Smart Grid, vol. 2, no. I, pp. 152-161, Mar. 2011.

[65] BUSONIU L., BABUSKA R., DE SCHUTTER B.: 'A comprehensive survey of multi-agent reinforcement learning', IEEE Trans Syst. Man. Cybern. C: Appl. Rev., 2008, 38, (2), pp. 156-172.

[66] WEISS G. (ED.): 'Multi-agent systems: a modern approach to distributed artificial intelligence' (MIT Press, Cambridge, MA, 1999).

[67] W. Ren and R. W. Beard, "Consensus seeking in multi-agent systems under dynamically changing interaction topologies," IEEE Trans. Autom. Control, vol. 50, no. 5, pp. 655-661, May 2005.

[68] W. Liu, W. Gu, B. Zhao, " A Noval Multi-Agent based Control Approach for Frequency Stabilization of Islanded Microgrids," 2013 4th IEEE PES Innovative Smart Grid Technologies Europe (ISGT Europe), pp. 1-5, Oct 2013.

[69] Gaurav. S. and Balakrishnan. S., "Adaptive critic based neurocontroller for autolanding of aircrafts", Proceedings of the American Control Conference, Vol 2, pp. 1081-1085, 1997.

[70] Ma. J., Yang. T., Hou. Z. G., "Dual Heuristic Programming Based Neurocontroller for Vibration Isolation Control”, IEEE Proceeding of International Conference on Networking, Sensing and Control, Sanya, pp. $874-879,2008$.

[71] Liu D. R., Xiong X., Zhang Y., “Action-dependent adaptive critic designs”, Proceedings of the INNS- IEEE International Joint Conference on Neural Networks, pp. 990-995, 2001. 
KESHTKAR: MODELING AND CONTROLLING A HYBRID MULTI-AGENT BASED MICROGRID IN PRESENCE OF DIFFERENT PHYSICAL AND CYBER COMPONENTS

[72] L. Jiaqi R.G. Harley, G.K. Venayagamoorthy, "Adaptive critic design based dynamic optimal power flow controller for a smart grid," Intelligence Applications in Computational In Smart Grid (CIASG), 2011 IEEE Symposium on, vol., no., pp.1-8, 11-15 April 2011

[73] A. Alimardani, H. Keshtkar, B. Abdi, “Optimization of rotor speed variation in Microturbines", Energy Procedia, Proceedings of International Conference on Smart Grid and Clean Energy Technologies (ICSGCE 2011), Vol.12, pp. 789-798, 2011.

[74] B. Vahidi, E. Nasr Azadani, P. Hassanpor Divshali, A. H. Hessaminia, S. H. Hosseinian, "Novel Approach for Determination of Worst Loading Direction and Fast Prediction of Stability Margin in Power Systems", Simulation, Vol. 86, No. 1, 2010.

[75] P. Hasanpor Divshali, S. H. Hosseinian, E. Nasr Azadani, M. Abedi, "Application of bifurcation theory in dynamic security constrained optimal dispatch in deregulated power system", Springer, Electrical Engineering, Vol. 93, Issue 3, pp 157-166, 2011.

[76] D. Kundur, X. Feng, S. Liu, T. Zourntos, and K. Butler-Purry, "Towards a framework for cyber attack impact analysis of the electric smart grid," in Proc. of IEEE Smart Grid Comm, Gaithersburg, MD, Oct. 2010.

[77] D. E.rnst, "Reinforcement Learning applied to Power System Oscillations Damping", Proceedings of the 40th IEEE Conference on Decision and Control Orlando, Florida USA, December 2001. 


\section{Chapter 3}

\section{Hierarchical Multi-Agent based Voltage and}

Frequency Control for a Microgrid Power System 
KESHTKAR: MODELING AND CONTROLLING A HYBRID MULTI-AGENT BASED MICROGRID IN PRESENCE OF DIFFERENT PHYSICAL AND CYBER COMPONENTS

In previous chapter modeling and frequency control of an isolated smart microgrid in a centralized multi-agent based infrastructure was studied. In this chapter, the modeled microgrid system is taken to another level of multi-agent modeling and control for frequency and node voltages. Each DG unit is considered as an agent and is modeled as an inverter based generation unit. Firstly, the modeling of the microgrid components including the inverter based DG units is presented. The stability issues of the modeled microgrid increases with wide penetraion of wind generation in addition to the rapid load changes in the system. Therefore, the stability of the modeled microgrid is investigated through small signal stability analysis. Secondly, the control algorithms used in a hierarchical multi-agent infrastructure are described. For example, Particle Swarm Optimization and Adaptive Dynamic Programing are introduced as tools to calculate the control parameters. The proposed control approaches and multi-agent structure are applied in the modeled inverter based microgrid and is tested against oscillations in the system. The results show improvement in control performance of the microgrid system using the proposed multi-agent based control method.

\section{I- INTRODUCTION}

Microgrids are small-scale power systems that includes distributed generators (DG) as energy sources and can operate in both on-grid and off-grid modes [1-5]. While the microgrid is operating in grid connected mode, the frequency of the system is dictated by the nominal frequency of the main grid. However, the main microgrid frequency problem is raised when it is working in islanded mode. Islanding process results in active power unbalance between generation and consumption units which, in turn, may cause frequency instability and requires control techniques. The voltage deviation from nominal value is also more significant while the microgrid is working in islanded mode and it requires researches about implementing control methods [5-6].

There are two main control processes that maintain the stability of the system in case of deviations, primary and secondary control. The primary control shares the active and reactive power among DGs based on their ratings. It is used to maintain the stability of the system [7-9]. However, there can still be small deviation from nominal value in steady state after applying primary control.

Therefore, secondary control is employed in order to restore the DG voltages and frequencies to their nominal value [10-11], [12-15]. The conventional secondary controls for microgrids assume a centralized structure that requires a complex communication network and results in reduction in the reliability of the system [10-13].

In order to utilize communication structure to imrpove the microgrid control implementation, multi agent method is proposed. Multi Agent is one of the adaptive and intelligent control techniques that had not found much attention in the control design of microgrids [16-19]. Recently, the multi-agent system was proposed and recognized as one of the effective solutions for coordinating the distributed units in islanded microgrids [20-22].

In this chapter, MAS based controllers are designed to do local control and central control. The agents are software abstractions that utilize advanced algorithms such as PSO for device controllers that utilize PI control or ADP method to find the controller parameters. The developed controls are tested on a microgrid. Firstly, small-signal stability of the inverter based microgrids is analyzed. For this purpose the microgrid system needs to be linearized and presented by state space model. Since the dynamics of DGs in microgrids are nonlinear and non-identical, input-output feedback linearization is used to transform the nonlinear heterogeneous dynamics of DGs to linear dynamics. Then, in order to propose the new multi-agent based control technique in the modeled microgrid, each DG is considered as an agent and DG outputs are communicated among their controllers. Non-linear dynamics of DGs are used in the modeling of multi-agent microgrid for accurate stability analysis of the proposed control approach. 
This chapter is organized as follows. Section II models different part of the inverter based microgrid and studies the stability of the system through small signal analysis. Multi Agent System (MAS) along with the proposed control method for this microgrid are discussed in section III. Particle Swarm Optimization (PSO) and Adaptive Dynamic Programing (ADP) algorithm for tuning control parameters are also discussed in this section. Case studies and simulation results and analysis are shown in section IV. The chapter is concluded in section $\mathrm{V}$.

\section{II- SMALL SIGNAL MODELING AND STABILITY ANALYSIS OF THE INVERTER-BASED MICROGRID}

The analysis of the small-signal stability of conventional power systems is well established, however the transient stability analysis and control of the inverter-based microgrid against different oscillations remains to be studied [23-24]. This section develops the modeling and analysis of autonomous operation of inverter-based microgrids.

Using a common reference frame, steady-state model of each part of the microgrid system is obtained and combined to form the complete model of the system. The switching effects of the inverters have been neglected and their control loops are modeled in details. Instead of algebraic impedance model, full dynamic modeling has been utilized for modeling the network due to high frequency modes existed in inverters. For small signal stability purpose, the microgrid model is linearized and the eigenvalues (modes) of the state-space system matrix are studied in order to imrpove the transient stability of the system by designing feedback controllers on appropriate signals. In the next section, nonlinear equations are utilized for dynamic modeling of the microgrid system.

\subsection{Modeling for Small Signal Analysis}

The modeling method utilized in this section divides the microgrid system into three different sub-system including DG, network and loads (Fig. 3.1). The DG inverter dynamic model consist of the power sharing component, LC filter, output inductor and voltage and current controller. Voltage and current controllers have high-frequency dynamics which are more significant during large disturbances. Network dynamics can be neglected in small-signal analysis of conventional power systems due to the small time constants of network comparing to those of rotating machines and their controls. However, microgrid model has inverter-based DGs whose response times are small so network dynamics can affect system stability.

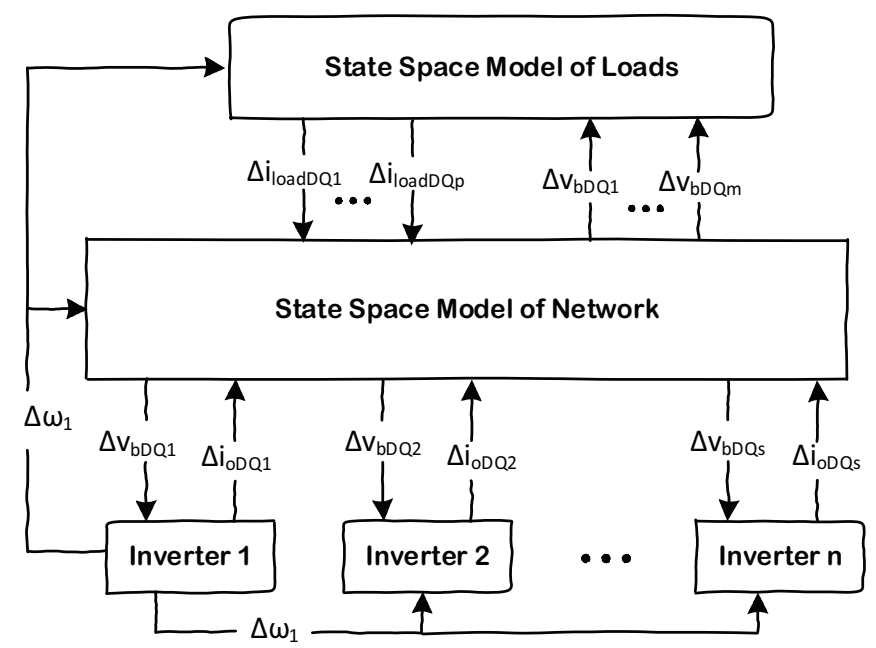

Fig. 3.1 Block diagram of complete small-signal state-space model of a microgrid. 
KESHTKAR: MODELING AND CONTROLLING A HYBRID MULTI-AGENT BASED MICROGRID IN PRESENCE OF DIFFERENT PHYSICAL AND CYBER COMPONENTS

\section{A. Model of a Voltage Source Inverter DGs}

The modeling of DG units has been changed in this chapter in order to implement voltage control along with frequency regulation. Conventionally, as it was discussed in previous section, the control parameters of the synchronous generator model are designed inside AGC, governor, and etc. But in the inverter based DG modeling which is going to be presented in this chapter the power controller, voltage controller, and current controller are performing inside the control block of the inverter to set the voltage and frequency reference of the three-leg inverter.

An average VSI model is mostly used as an interfacing module for each DG unit connecting to the network. Fig. 3.2 shows the block diagram of a VSI DG connected to a microgrid. A three-leg VSI with an LC filter and a coupling inductor form the power circuit, whereas three control loops form the control structure. DC bus dynamics can be neglected by assuming an ideal source from the DG side. The switching actions of the inverter can also be neglected in the modeling due to the high switching frequencies (about $10 \mathrm{kHz}$ ). A power controller is utilized in DG modeling to produce the magnitude and frequency of the inverter output voltage based on the droop characteristic and emulating the operation of a conventional synchronous generator. Voltage controller is utilized in DG model in order to use the reference filter-inductor current vector for generating the reference current signals and current controller utilizes these input signals to generate the command voltage signal to be used by a pulse-width-modulation (PWM) module [25]. Voltage and current control loops are performing to create enough damping for the output LC filter and coupling inductor. The role of output coupling inductor is to define the output impedance of the inverter in order to minimize the active-reactive power coupling.

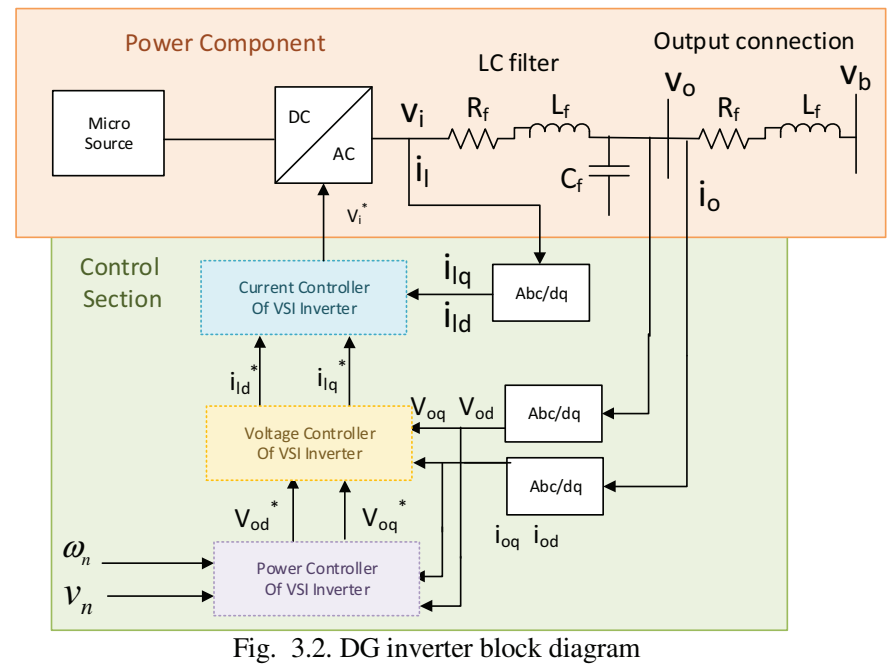

The current and voltage dynamic equations of the DG inverter model can be transferred to d-q frame which rotates synchronously with the inverter output voltage angular speed $\omega_{\mathrm{o}}$. Therefore, voltage and current dynamic equations are obtained as follows [25]:

$$
\begin{gathered}
\frac{d i_{l d}}{d t}=\frac{-r_{f}}{L_{f}} i_{l d}+\omega i_{l q}+\frac{1}{L_{f}} v_{i d}-\frac{1}{L_{f}} v_{o d} \\
\frac{d i_{l q}}{d t}=\frac{-r_{f}}{L_{f}} i_{l q}-\omega i_{l d}+\frac{1}{L_{f}} v_{i q}-\frac{1}{L_{f}} v_{o q} \\
\frac{d v_{o d}}{d t}=\omega v_{o q}+\frac{1}{C_{f}} i_{l d}-\frac{1}{C_{f}} i_{o d}
\end{gathered}
$$




$$
\begin{aligned}
& \frac{d v_{o q}}{d t}=-\omega v_{o d}+\frac{1}{C_{f}} i_{l q}-\frac{1}{C_{f}} i_{o q} \\
& \frac{d i_{o d}}{d t}=\frac{-r_{c}}{L_{c}} i_{o d}+\omega i_{o q}+\frac{1}{L_{c}} v_{o d}-\frac{1}{L_{c}} v_{b d} \\
& \frac{d i_{o q}}{d t}=\frac{-r_{c}}{L_{c}} i_{o q}-\omega i_{o d}+\frac{1}{L_{c}} v_{o q}-\frac{1}{L_{c}} v_{b q}
\end{aligned}
$$

$v_{\text {id }}, v_{\text {iq }}, i_{l d}$, and $i_{\text {lq }}$ are the DG inverter's voltages and currents; $v_{\text {od }}, v_{\text {oq }}, i_{\text {od }}$, and $i_{\text {oq }}$ are the DG output voltages and currents; $\mathrm{V}_{\mathrm{bd}}$ and $\mathrm{V}_{\mathrm{bq}}$ are the $\mathrm{d}$ - and $\mathrm{q}$-axis bus voltages, $\mathrm{r}_{\mathrm{f}}, \mathrm{L}_{\mathrm{f}}$, and $\mathrm{C}_{\mathrm{f}}$ are the per-phase resistance, inductance, and capacitance of the $\mathrm{LC}$ filter, respectively; and $r_{c}$ and $L_{c}$ are the per-phase resistance and inductance of the coupling inductor, respectively.

As shown in Fig. 3.3, utilizing the two-axis theory, the injected instantaneous active and reactive power components, p and q, are given by $p=v_{o d} i_{o d}+v_{o q} i_{o q}$ and $q=v_{o d} i_{o q}-v_{o q} i_{o d}$.

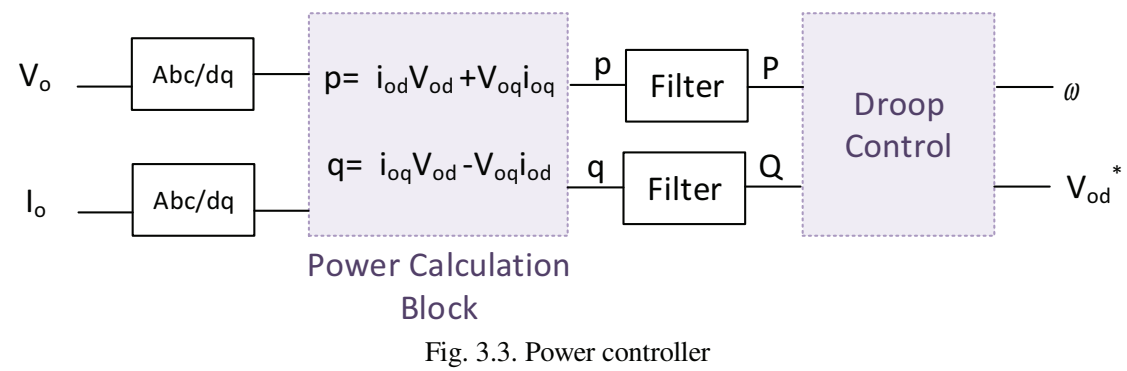

In order to obtain high power quality injection and also to create enough time-scale separation between the power and current controller loops, control actions are applied to the average active and reactive powers corresponding to the fundamental components which are obtained by filtering through $P=\frac{\omega_{c}}{s+\omega_{c}} p$ and $Q=\frac{\omega_{c}}{s+\omega_{c}} q \cdot \omega_{c}$ is the filter cutoff frequency of the filter.

Conventional droop characteristics are usually modeled in paralleled inverter systems to introduce the following droops in the output voltage and frequency of the DG:

$$
\begin{aligned}
& \omega_{o}=\omega^{*}-m P \\
& v_{o d}^{*}=V^{*}-n Q
\end{aligned}
$$

In (3.7) and (3.8), $\omega^{*}$ and $\mathrm{V}^{*}$ are the nominal frequency and voltage set points, respectively, and $\mathrm{m}$ and $\mathrm{n}$ are the droop gains, which are calculated based on the range of frequency and voltage changes using $m=\frac{\omega_{\max }-\omega_{\min }}{P_{\max }}$ and $n=\frac{V_{o d \max }-V_{o d \min }}{Q_{\max }}$.

$\omega^{*}$ and $\mathrm{V}^{*}$ in the above equations perform as virtual communication agents for different DG inverters for autonomous operation. Also, since q-component of the output voltage is zero, $v_{\text {od }}$ is utilized in (3.8) for voltage-oriented control and the reference of the output voltage magnitude is aligned with the d-axis of the DG reference frame.

For voltage regulation purposes, DG output voltage control is implemented which is shown in Fig. 3.4. The proportionalintegral (PI) controller is used along with feedforward and decoupling control loops to provide the reference current. The dynamics of the voltage controller can be presented by

$$
\begin{gathered}
i_{l d}^{*}=F i_{o d}-\omega_{n} C_{f} v_{o q}+K_{p v}\left(v_{o d}^{*}-v_{o d}\right)+K_{i v} \phi_{d} \\
i_{l q}^{*}=F i_{o q}+\omega_{n} C_{f} v_{o d}+K_{p v}\left(v_{o q}^{*}-v_{o q}\right)+K_{i v} \phi_{q}
\end{gathered}
$$

In (3.9) and (3.10) $\mathrm{K}_{\mathrm{pv}}, \mathrm{K}_{\mathrm{iv}}$ are the proportional and integral gains, respectively, and $\mathrm{C}_{\mathrm{f}}$ is the filter capacitance. 


\section{KESHTKAR: MODELING AND CONTROLLING A HYBRID MULTI-AGENT BASED MICROGRID IN PRESENCE OF DIFFERENT PHYSICAL AND CYBER COMPONENTS}

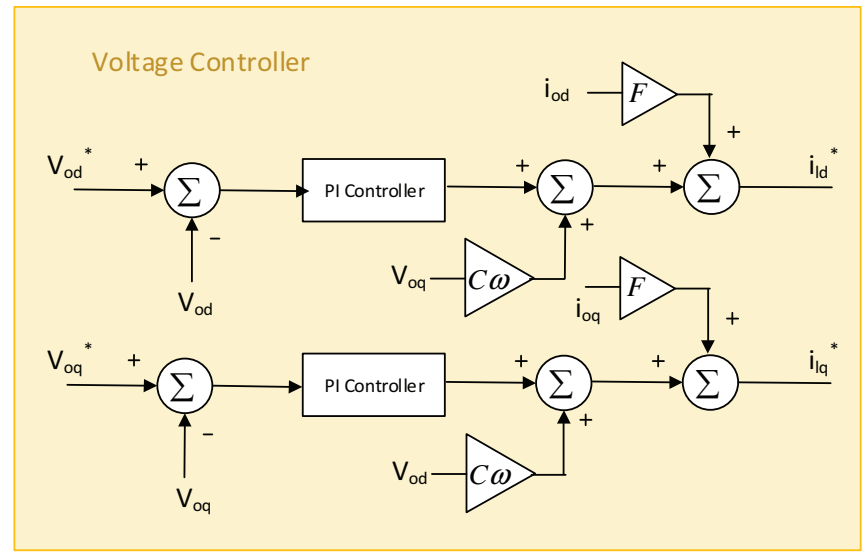

Fig. 3.4. Voltage controller

In order to create the voltage across the filter inductor, a current controller is required to minimize the current error. Fig. 3.5 shows the current controller structure. A PI regulator with feedforward and decoupling control loops is utilized for current regulation purpose. The dynamics of the current controller is obtained by

$$
\begin{gathered}
v_{i d}^{*}=-\omega_{n} L_{f} i_{l q}+K_{p c}\left(i_{l d}^{*}-i_{l d}\right)+K_{i c} \gamma_{d} \\
v^{*}{ }_{i q}=\omega_{n} L_{f} i_{l d}+K_{p c}\left(i_{l q}^{*}-i_{l q}\right)+K_{i c} \gamma_{q}
\end{gathered}
$$

In (3.11) and (3.12) $\mathrm{K}_{\mathrm{pc}}, \mathrm{K}_{\mathrm{ic}}$ are the proportional and integral gains of the PI regulator, respectively.

It is worth to mention that more advanced current and voltage controls can be implemented in the DG model [26]; however, conventional PI-based voltage and current controls are applied in this section, where the main scope is small signal modeling and stability analysis.

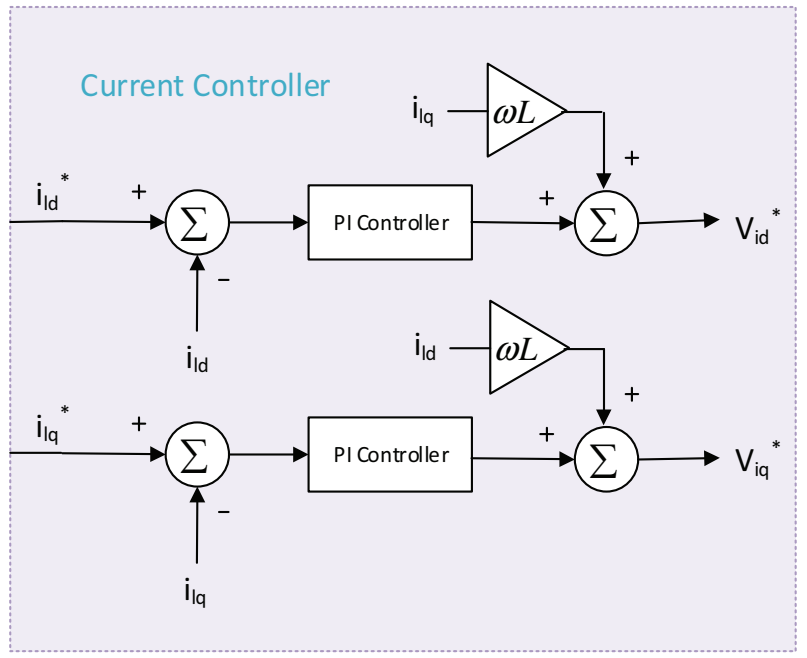

Fig. 3.5. Current controller

Equations (3.1)-(3.12) models the dynamic behavior of a single inverter-based DG unit with the conventional droop controller. Small signal analysis is utilized as a useful tool for analyzing the dynamic performance and implementing the control system [27]; the aforementioned equations can be linearized and the following state-space model of a single inverter-based DG unit can be calculated by (3.13) and (3.14).

$$
\Delta \dot{x}_{D G}=A_{D G} \Delta x_{D G}+B_{D G} \Delta v_{s}
$$




$$
\Delta x_{D G}=\left[\begin{array}{llllllll}
\Delta \delta & \Delta P & \Delta Q & \Delta C_{V} & \Delta C_{C} & \Delta i_{d q} & \Delta v_{o d q} & \Delta i_{o d q}
\end{array}\right]^{T}
$$

In the state space model given by (3.13) and (3.14), $\delta$ represents the angle between the inverter-based DG reference frame and a common reference frame; $\mathrm{C}_{\mathrm{V}}$ and $\mathrm{C}_{\mathrm{C}}$ are the integrator states of the voltage and current controllers, respectively; $\mathrm{v}_{\mathrm{s}}$ is the supply voltage at the connection point to the rest of the microgrid which is transferred to a common reference frame; and $A_{D G}$ and $B_{D G}$ are the state and input matrices, and they are given as presented in (3.15). All the initial values of state variables before any disturbance in the system are set to zero.

\section{B. Combined Model of All the Inverters}

Previously, the small-signal modelling of an individual DG inverter on a common reference frame was discussed. In a microgrid there can be several inverters acting as sources and connected remotely from each other. The modelling approach in this section is to form a sub-model of all the individual DG inverters and combine them with the network and individual load models.

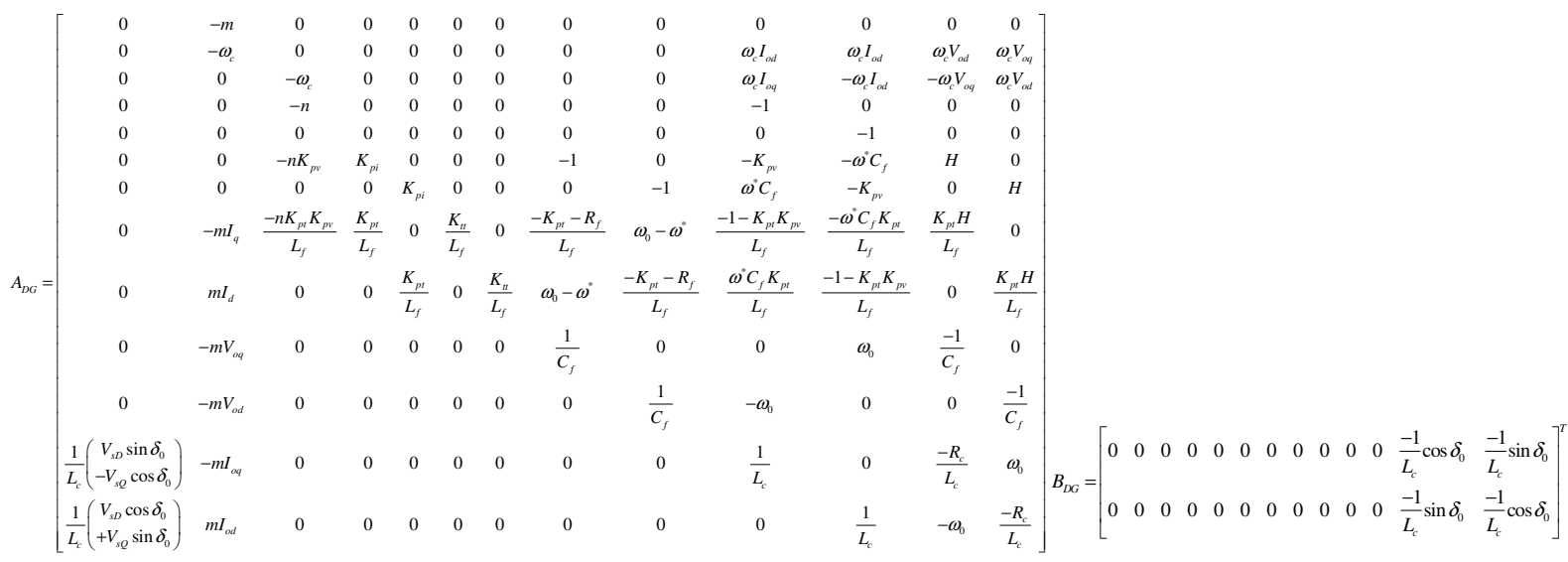

Let us consider a system with "s" number of DG inverters where the reference frame of inverter number 1 is taken as the common reference frame. Then, from previous section, a combined small-signal model of all the inverter units together is obtained, as shown in (3.16)-(3.18).

$$
\begin{aligned}
& {\left[\Delta \dot{x}_{I N V}\right]=A_{I N V}\left[\Delta x_{I N V}\right]+B_{I N V}\left[\Delta v_{b D Q}\right]} \\
& {\left[\Delta i_{o D Q}\right]=C_{I N V}\left[\Delta x_{I N V}\right]}
\end{aligned}
$$

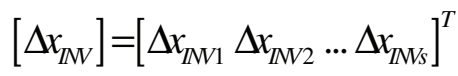

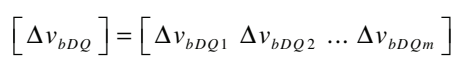

The state vector $\left[\Delta x_{i n v}\right]$ is shown in (3.17) and the input vector $\left[\Delta v_{b D Q}\right]$ is shown in (3.18). The state space matrices, $\mathrm{A}_{\mathrm{INV}}$, $\mathrm{B}_{\mathrm{INV}}$, and $\mathrm{C}_{\mathrm{INV}}$ are given in Appendix section.

\section{Network Model}

An example network of $\mathrm{n}$ lines and $\mathrm{m}$ nodes with $\mathrm{s}$ inverters and $\mathrm{p}$ load points is shown in Fig. 3.6. On a common reference frame the state equations of line current of $\mathrm{i}^{\text {th }}$ line connected between nodes $\mathrm{j}$ and $\mathrm{k}$ are 
KESHTKAR: MODELING AND CONTROLLING A HYBRID MULTI-AGENT BASED MICROGRID IN PRESENCE OF DIFFERENT PHYSICAL AND CYBER COMPONENTS

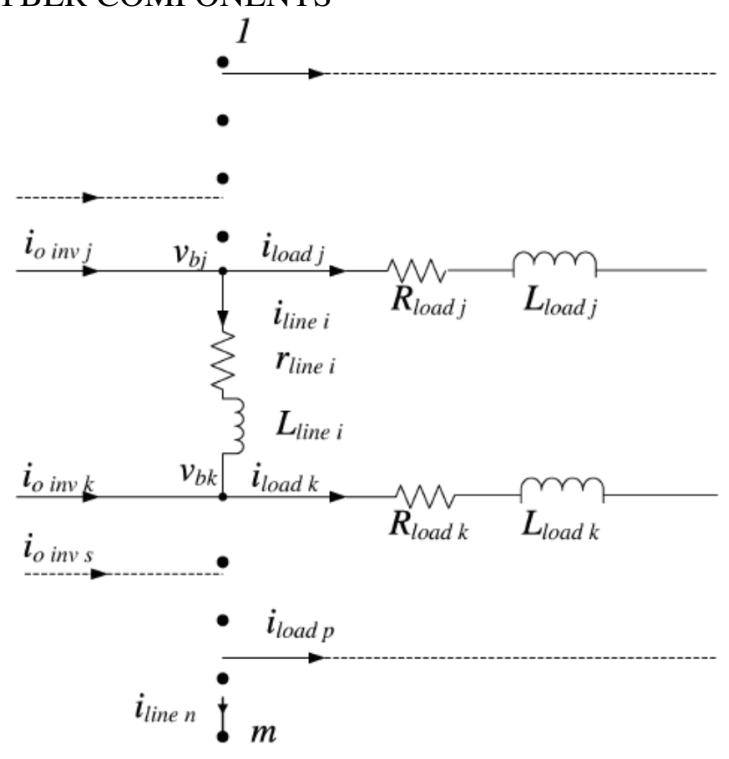

Fig. 3.6. Network representation

$$
\begin{aligned}
& \frac{d i_{\text {line } D i}}{d t}=\frac{-r_{\text {linei }}}{L_{\text {linei }}} i_{\text {line Di }}+\omega i_{\text {line } Q i}+\frac{1}{L_{\text {linei }}} v_{b D j}-\frac{1}{L_{\text {linei }}} v_{b D k} \\
& \frac{d i_{\text {line } Q}}{d t}=\frac{-r_{\text {linei }}}{L_{\text {linei }}} i_{\text {line } Q i}-\omega i_{\text {lineDi } i}+\frac{1}{L_{\text {linei }}} v_{b Q j}-\frac{1}{L_{\text {linei }}} v_{b Q k}
\end{aligned}
$$

Hence, the small-signal state-space model of a network with lines is given by (3.21)-(3.23).

$$
\begin{gathered}
{\left[\Delta i_{\text {lineDQ }}^{\cdot}\right]=A_{N E T}\left[\Delta i_{\text {lineDQ }}\right]+B_{1 N E T}\left[\Delta v_{b D Q}\right]+B_{2 N E T} \Delta \omega} \\
{\left[\Delta i_{\text {line } D Q}\right]=\left[\Delta i_{\text {line } D Q 1} \Delta i_{\text {line } D Q 2} \ldots \Delta i_{\text {line } D Q n}\right]^{T}} \\
{\left[\Delta v_{b D Q}\right]=\left[\begin{array}{lll}
\Delta v_{b D Q 1} & \left.\Delta v_{b D Q 2} \ldots \Delta v_{b D Q m}\right]^{T}
\end{array}\right.}
\end{gathered}
$$

$\Delta \omega$ in (3.21) is $\Delta \omega_{c o m}$ and the state space matrices, $\mathrm{A}_{\mathrm{NET}}, \mathrm{B}_{1 \mathrm{NET}}$, and $\mathrm{B}_{2 \mathrm{NET}}$ are given in Appendix section.

\section{Load Model}

Although, many types of load can exist in microgrids, a general RL load is considered in this section. The state equations of the RL load connected at $\mathrm{i}^{\text {th }}$ node are

$$
\begin{aligned}
& \frac{d i_{\text {laodDi }}}{d t}=\frac{-R_{\text {loadi }}}{L_{\text {loadi }}} i_{\text {loadDi }}+\omega i_{\text {loadQi }}+\frac{1}{L_{\text {loadi }}} v_{b D i} \\
& \frac{d i_{\text {laodQi }}}{d t}=\frac{-R_{\text {loadi }}}{L_{\text {loadi }}} i_{\text {loadQi }}+\omega i_{\text {loadDi }}+\frac{1}{L_{\text {loadi }}} v_{b Q i}
\end{aligned}
$$

Hence, for a network with load points the small-signal state space model of loads is given by (3.26)

$$
\left[\Delta i_{\text {loadDQ }}^{\cdot}\right]=A_{\text {load }}\left[\Delta i_{\text {loadDQ }}\right]+B_{1 L O A D}\left[\Delta v_{b D Q}\right]+B_{2 L O A D} \Delta \omega
$$

The state space matrices in (3.26), $\mathrm{A}_{\text {load }}, \mathrm{B}_{\text {lload, }}$ and $\mathrm{B}_{2 \text { load }}$ are given in Appendix section. 


\section{E. Complete Microgrid Model}

In order to make sure that the node voltage is well defined (and that the numerical solution well-conditioned) a virtual resistor $r_{N}$ is assumed between each node and ground. The value of virtual resistor is chosen sufficiently large so it would have minimum influence on the dynamic stability of the system. Hence, the voltage of the node is given by

$$
\begin{aligned}
& v_{b D i}=r_{N}\left(i_{o D i}-i_{\text {load } D i}+i_{\text {lineDi,j }}\right) \\
& v_{b Q i}=r_{N}\left(i_{o Q i}-i_{\text {load } Q i}+i_{\text {line } Q i, j}\right)
\end{aligned}
$$

In symbolic form, for a network with m nodes

$$
\left[\Delta v_{b D Q}\right]=R_{N}\left(M_{I N V}\left[\Delta i_{o D Q}\right]+M_{\text {load }}\left[\Delta i_{\text {load } Q}\right]+M_{N E T}\left[\Delta i_{\text {lineDQ }}\right]\right)
$$

In (3.29), $R_{N}$ is a $2 m * 2 m$ matrix with diagonal elements equal to $r_{N}$. $M_{I N V}$ is $2 m * 2 s$ mapping matrix, which relates the inverter connection points to network nodes. For instance, if the inverter $\mathrm{j}$ is connected at the node $\mathrm{i}$, the element $\mathrm{M}_{\mathrm{INV}}(\mathrm{j}, \mathrm{i})$ will be 1 and all the other elements in row $\mathrm{j}$ will be equal to 0 . Similarly, $\mathrm{M}_{\text {load }}$ is $2 \mathrm{~m} * 2 \mathrm{p}$ matrix that maps load connection points onto the network nodes with -1 . Matrix $\mathrm{M}_{\mathrm{NET}}$ is a $2 \mathrm{~m} * 2 \mathrm{n}$ matrix which maps the connecting lines onto the network nodes. Either +1 or -1 is chosen based on whether the given line current is entering or leaving the node, respectively. Now, the complete microgrid smallsignal state-space model and hence the system state matrix [as given in (3.30)] can be calculated by using the individual subsystem models given by (3.16), (3.21), and (3.26)

$$
\left[\begin{array}{c}
\Delta \dot{x}_{I N V} \\
\Delta i_{\text {lineDQ }} \\
\Delta i_{\text {load } Q}
\end{array}\right]=A_{m g}\left[\begin{array}{c}
\Delta x_{I N V} \\
\Delta i_{\text {lineDQ }} \\
\Delta \dot{l}_{\text {loadDQ }}
\end{array}\right]
$$

The complete system state matrix $\mathrm{A}_{\mathrm{mg}}$ is given in (3.31). The small-signal flow among all sub-modules is presented in Fig. 3.1.

$$
A_{m g}=\left[\begin{array}{ccc}
A_{I N V}+B_{I N V} R_{N} M_{I N V} C_{I N V C} & B_{I N V} R_{N} M_{N E T} & B_{I N V} R_{N} M_{\text {load }} \\
B_{\text {INET }} R_{N} M_{I N V} C_{I N V C}+B_{2 N E T} C_{I N V \omega} & A_{N E T}+B_{1 N E T} R_{N} M_{N E T} & B_{1 N E T} R_{N} M_{\text {load }} \\
B_{1 L O A D} R_{N} M_{I N V} C_{I N V C}+B_{2 L O A D} C_{I N V \omega} & B_{1 L O A D} R_{N} M_{N E T} & A_{\text {load }}+B_{1 L O A D} R_{N} M_{\text {load }}
\end{array}\right]
$$

The difference between the steady space modeling of the microgrid which is presented in this part and the model of a distribution or transmission power system is based on the difference between their DG modeling. In fact, the network and load modeling process are similar. Furthermore, having less inertia, integration of distributed generation, and ability to work in islanded mode from the main grid are the other differences in modeling microgrid and larger power systems.

\subsection{Small Signal Stability Analysis}

The linearized model described earlier is used for an inverter based microgrid. The small signal stability analysis and design of a simple load frequency control is discussed in this part.

\section{A. Case study}

The microgrid case study for developing the state space model is shown in Fig. 3.7. 


\section{KESHTKAR: MODELING AND CONTROLLING A HYBRID MULTI-AGENT BASED MICROGRID IN PRESENCE} OF DIFFERENT PHYSICAL AND CYBER COMPONENTS

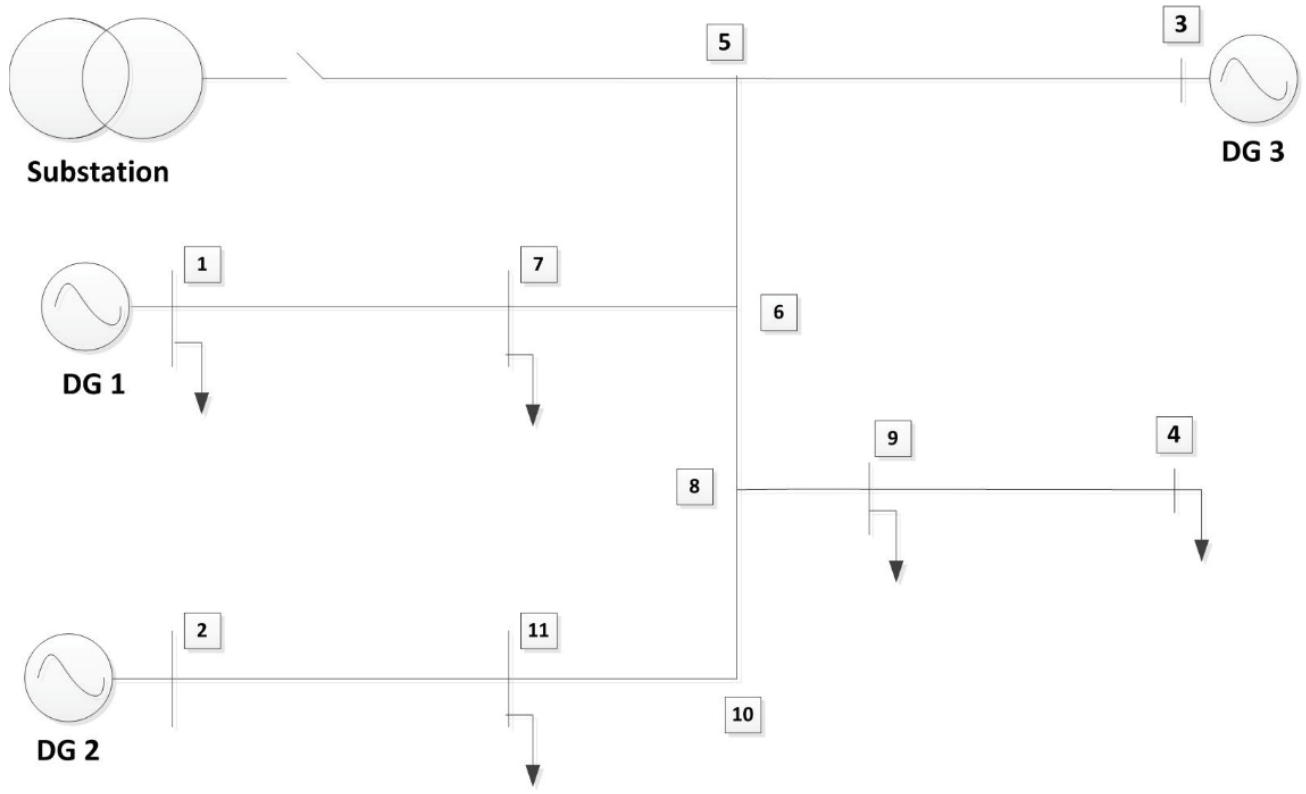

Fig. 3.7. Microgrid case study

The line data and parameters are obtained from IEEE 13 node system and total generation and loads are presented in the modeled microgrid in [28].

\section{B. Analysis}

The eigenvalues of the final matrix of the whole microgrid $\left(\mathrm{A}_{\mathrm{mg}}\right)$ are shown in Fig. 3.8. It can be seen that the eigenvalues are located on the left-hand side of the imaginary axis that confirm the stable dynamics of the system. Small eigenvalues have more influence on the transient behavior of the system and will be relocated by feedback controllers to improve the stability of the system. Therefore, based on the results, the best feedback signals for frequency control design in part B.3 are selected. Here, with applying a load change in the system the response of the system is observed.

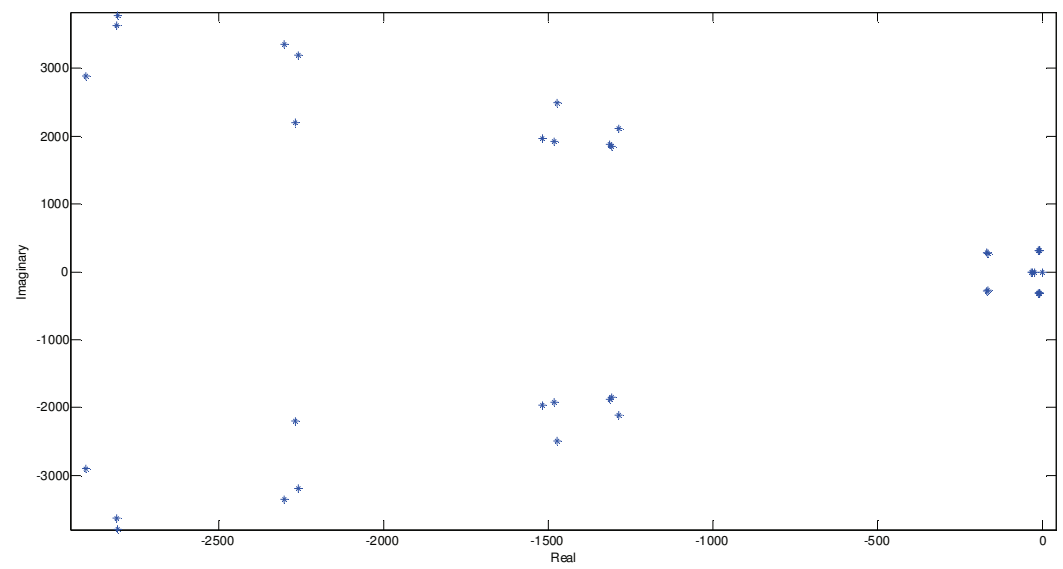

Fig. 3.8. The eigenvalues of the test system

$10 \%$ load change is applied on node 10 at $\mathrm{t}=0 \mathrm{~s}$ and the inverter currents and frequency (rotational speed) deviation, and node voltages (d\&q axis) deviation are monitored and presented in Fig. 3.9-3.12. 


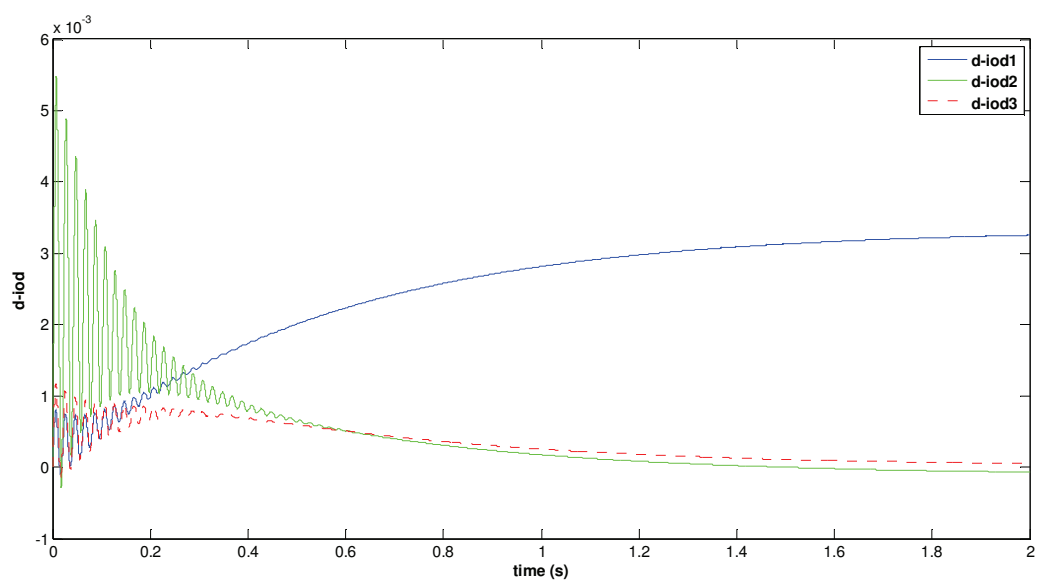

Fig. 3.9. Inverters output $d$ currents deviations (after load change)

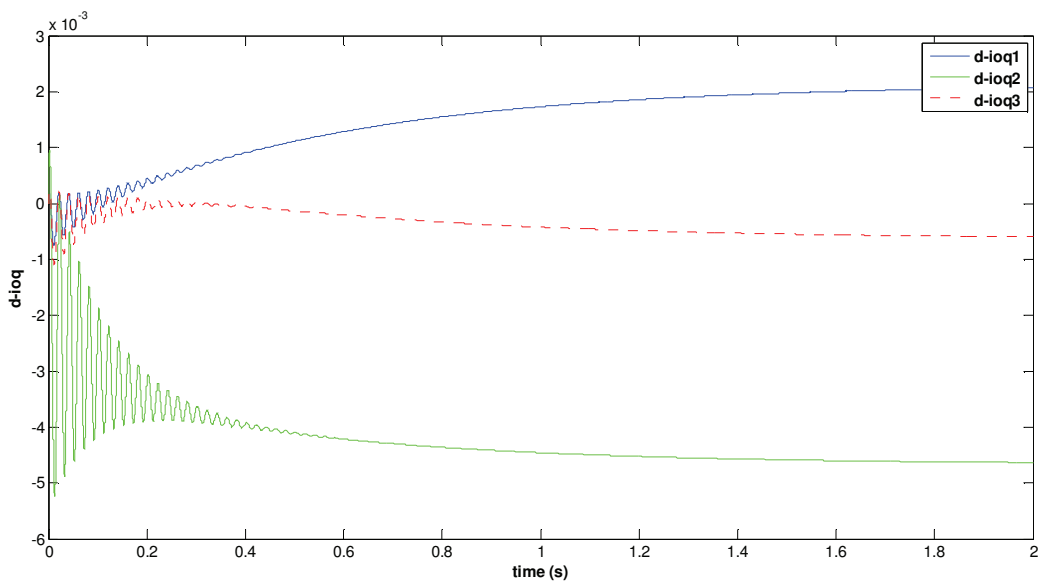

Fig. 3.10. Inverters output q currents deviations (after load change)

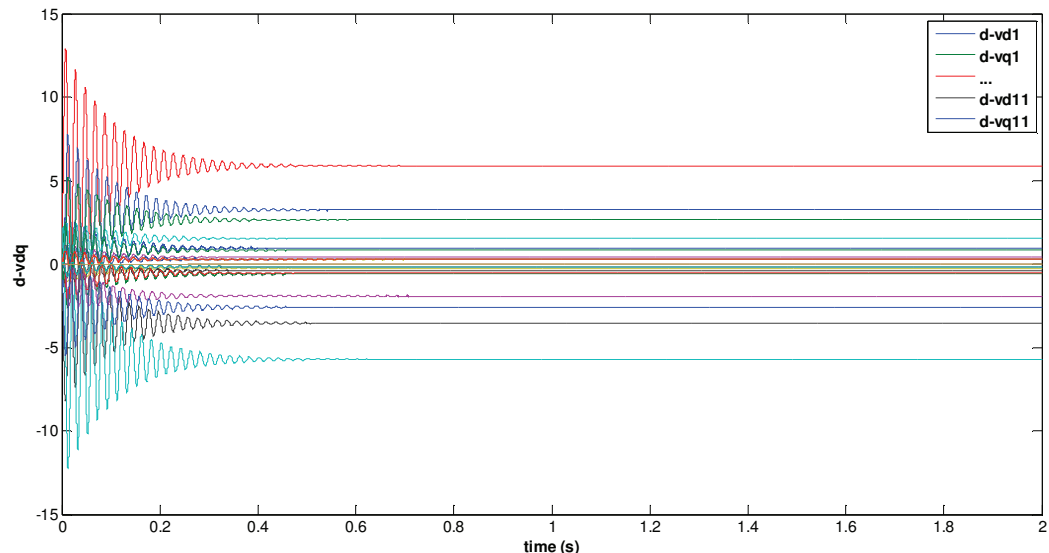

Fig. 3.11. Nodes d \& q voltages deviation (after load change) 


\section{KESHTKAR: MODELING AND CONTROLLING A HYBRID MULTI-AGENT BASED MICROGRID IN PRESENCE OF DIFFERENT PHYSICAL AND CYBER COMPONENTS}

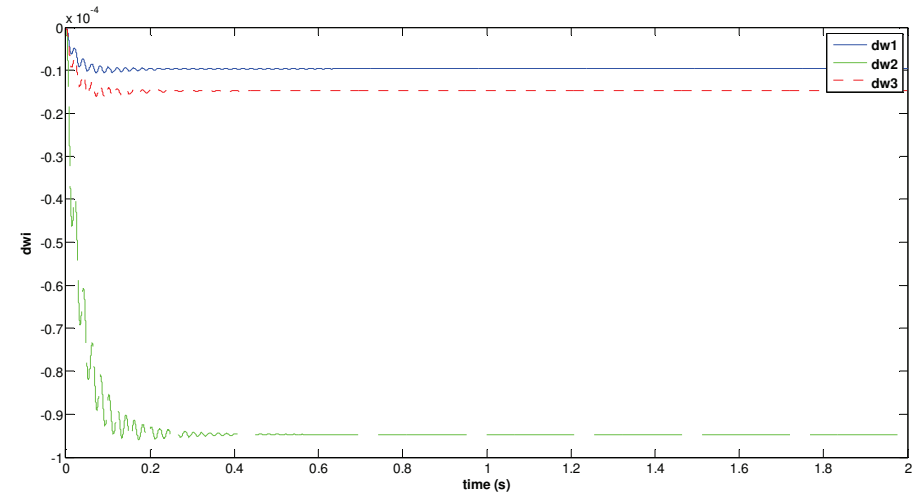

Fig. 3.12 Inverters frequencies (rotational speed) deviation (after load change)

It can be seen from Fig. 3.9, 3.10 and 3.12 that the frequency and currents of the second inverter, which is related to the closest DG (Wind Generation) unit (at node 2) with respect to the load change location at node 10 (see Fig. 3.7), deviates more than the other inverter frequencies. This fact can also be seen in the voltages of the closer nodes to the fault locations in Fig. 3.11. In the next step of this section a simple controller is added to the system to restore the frequency of the system to the nominal value.

\section{LFC design}

Droop method is conventionally used as a robust control strategy against p-f oscillations [37]. However, restoring the deviated frequency to nominal value after load changes needs secondary load-frequency control [34]. One method for implementation of this method is to utilize a central control unit which communicate with all the DGs to provide the reference control signal. The other method is to design a local controller for each DG of the microgrid system [37]. This distributed approach requires less communication links since the controllers only need the local measurements. In this part, decentralized method is implemented through designing PI controller for each DG. The dynamic aspects of the DG units are neglected in the small signal stability analysis due to the steady-state implementation of droop control technique. In this case, 5\% droop and integral gain of 1 is utilized for each DG ( $\mathrm{Ri}=0.05 \& \mathrm{Ki}=1)$. The frequency (rotational speed) deviation responses for three DGs are shown in Fig. 3.13.

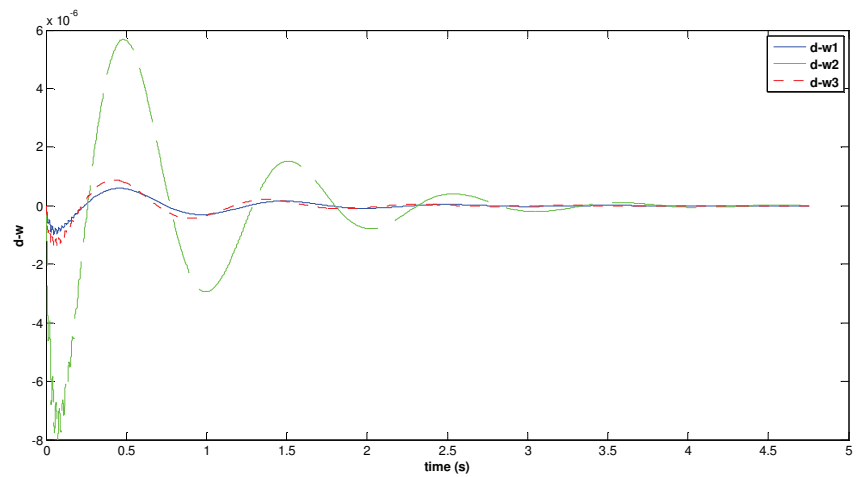

Fig. 3.13. Inverters frequencies (rotational speed) deviations (after LFC)

Reference frequencies of DGs are provided as nominal frequency of the system and the deviation from this value is shown in Fig. 3.13. The frequencies have overshoots and deviations due to the load change at the initial transient, however, it is restored to the nominal value by the implemented LFC. These oscillations can also be reduced by tuning the parameters of the controllers by optimization algorithms, which is going to be discussed in the next section. It can be seen that the frequency of the second inverter, which is related to the closest DG (Wind Generation) unit (at node 2) with respect to the load change location at node 10 (see Fig. 
3.7), deviates more than the other inverter frequencies. Therefore, the designed LFC is capable of damping the frequency oscillations due to the disturbances in the microgrid. Also, it can be observed from the currents and voltages curves that these signals take different steady-state values for each node based on the new load condition. And similar to frequency, the voltages and currents of the nodes which ate closer to the load change node have more deviation from their nominal values.

\section{III- MODELING OF MULTI AGENT BASED CONTROL OF MICROGRID}

The linear modeling of the physical layer of the microgrid was described in the previous section for stability analysis purpose. This section discusses the nonlinear dynamic model of the microgrid and then the multi-agent based control algorithm which is used is implemented in the cyber layer of the microgrid.

\subsection{Dynamic Model of Inverter-based Microgrid}

The proposed secondary voltage control which is presented in the rest of this section is designed based on nonlinear model of the microgrid. Therefore, the nonlinear dynamical model of the DG is discussed here. The block diagram of an inverter-based DG was shown in Fig. 3.1. It contains an inverter bridge, connected to a primary dc power source (e.g., photovoltaic panels or fuel cells). The control loops, including the power, voltage, and current controllers, adjust the output voltage and frequency of the inverter bridge [28]-[30]. Given the relatively high switching frequency of the inverter bridge, the switching artifacts can be safely neglected via average-value modeling. As stated in [28], dc-bus dynamics can be safely neglected, assuming an ideal source from the DG side.

It should be noted that the nonlinear dynamics of each DG are formulated in its own d-q (direct-quadrature) reference frame [38]. It is assumed that the reference frame of the ith DG is rotating at the frequency of $\omega_{\mathrm{i}}$. The reference frame of one DG is considered as the common reference frame with the rotating frequency of $\omega_{\text {com. }}$. The angle of the ith DG reference frame, with respect to the common reference frame, is denoted as $\delta_{\mathrm{i}}$ and satisfies the following differential equation:

$$
\dot{\delta}=\omega_{l}-\omega_{c o m}
$$

Although different angular frequencies are considered for reference frames, all of the reference frames rotate synchronously at a common angular frequency due to the presence of the frequency-droop characteristic in (3.7).

The power controller block, shown in Fig. 3.3, contains the droop technique in (3.7) and provides the voltage references $v_{\text {odi }}^{*}$ and $v_{o q i}^{*}$ for the voltage controller, as well as the operating frequency $\omega_{\mathrm{i}}$ for the inverter bridge. Two low-pass filters with the cutoff frequency of $\omega_{\mathrm{ci}}$ are used to extract the fundamental component of the output active and reactive powers denoted as $P_{\mathrm{i}}$ and $\mathrm{Q}_{\mathrm{i}}$, respectively. The differential equations of the power controller can be written as

$$
\begin{aligned}
& \dot{P}_{i}=-\omega_{c i} P_{i}+\omega_{c i}\left(v_{o d i} i_{o d i}+v_{o q i} i_{o q i}\right) \\
& \dot{Q}_{i}=-\omega_{c i} Q_{i}+\omega_{c i}\left(v_{o q i} i_{o d i}-v_{o d i} i_{o q i}\right)
\end{aligned}
$$

Where $v_{\text {odi }}, v_{o q i}, i_{o d i}$, and $i_{o q i}$ are the direct and quadrature components of $v_{o i}$ and $i_{o i}$ in Fig. 3.3. As seen in Fig. 3.4, the primary voltage control strategy for each DG aligns the output voltage magnitude on the d-axis of the corresponding reference frame. Therefore, $v_{o d i}^{*}=V_{n i}-n_{Q i} Q_{i}$ and $v_{o q i}^{*}=0$.

The block diagram of the voltage controller is shown in Fig. 3.4 [38]. The differential algebraic equations of the voltage controller are written as (3.35)-(3.38).

$$
\dot{\phi}_{d i}=v_{o d i}^{*}-v_{o d i}
$$


KESHTKAR: MODELING AND CONTROLLING A HYBRID MULTI-AGENT BASED MICROGRID IN PRESENCE OF DIFFERENT PHYSICAL AND CYBER COMPONENTS

$$
\begin{gathered}
\dot{\phi}_{q i}=v_{o q i}^{*}-v_{o q i} \\
i_{l d i}^{*}=F_{i} i_{o d i}-\omega_{b} C_{f i} v_{o q i}+K_{P V i}\left(v_{o d i}^{*}-v_{o d i}\right)+K_{I V i} \phi_{d i} \\
i_{l q i}^{*}=F_{i} i_{o q i}+\omega_{b} C_{f i} v_{o d i}+K_{P V i}\left(v_{o q i}^{*}-v_{o q i}\right)+K_{I V i} \phi_{q i}
\end{gathered}
$$

In the above equations $\phi_{\mathrm{di}}$ and $\phi_{\mathrm{qi}}$ are the auxiliary state variables defined for PI controllers in Fig. 3.4. $\omega_{\mathrm{b}}$ is the nominal angular frequency. Other parameters are shown in Fig. 3.2 and 3.4 and discusses in section II.A.1.

The block diagram of the current controller is shown in Fig. 3.5 [38]. The differential algebraic equations of the current controller are written as

$$
\begin{gathered}
\dot{\gamma}_{d i}=i_{l d i}^{*}-i_{l d i} \\
\dot{\gamma}_{q i}=i_{l q i}^{*}-i_{l q i} \\
v_{i d i}^{*}=-\omega_{b} L_{f i} i_{l q i}+K_{P C i}\left(i_{l d i}^{*}-i_{l d i}\right)+K_{I C i} \gamma_{d i} \\
v_{i q i}^{*}=\omega_{b} L_{f i} i_{l d i}+K_{P C i}\left(i_{l q i}^{*}-i_{l q i}\right)+K_{I C i} \gamma_{q i}
\end{gathered}
$$

In the above equations $\gamma_{\mathrm{di}}$ and $\gamma_{\mathrm{qi}}$ are the auxiliary state variables defined for the PI controllers in Fig. 3.5 and $i_{\text {ldi }}$ and $i_{\text {lqi }}$ are the direct and quadrature components of $\mathrm{i}_{\mathrm{li}}$ in Fig. 3.3. Other parameters are shown in Figs. 3.2 and 3.5 and discusses in section II.A.1.

The differential equations for the output LC filter and output connector are the same as (3.1)-(3.6). These equations form the large-signal dynamical model of the $\mathrm{i}^{\text {th }} \mathrm{DG}$. The large-signal dynamical model can be written in a compact form as (3.43) and (3.44).

$$
\begin{aligned}
& \left\{\begin{array}{l}
\dot{X}_{i}=f_{i}\left(X_{i}\right)+k_{i}\left(X_{i}\right) D_{i}+g_{i}\left(X_{i}\right) u_{i} \\
y_{i}=h_{i}\left(X_{i}\right)
\end{array}\right. \\
& X_{i}=\left[\begin{array}{lllllllllllll}
\delta_{i} & P_{i} & Q_{i} & \phi_{d i} & \phi_{q i} & \gamma_{d i} & \gamma_{q i} & i_{l d i} & i_{l q i} & v_{o d i} & v_{o q i} & i_{o d i} & i_{o q i}
\end{array}\right]^{T}
\end{aligned}
$$

The term $D_{i}=\left[\omega_{c o m} v_{b d i} v_{b q i}\right]^{T}$ is considered as a known disturbance. The detailed expressions for $f_{i}\left(X_{i}\right), g_{i}\left(X_{i}\right), k_{i}\left(X_{i}\right)$, and $h_{i}\left(X_{i}\right)$ can be extracted from above equations.

The secondary voltage control selects $\mathrm{V}_{\mathrm{ni}}$ in (3.8) such that the terminal voltage amplitude of each DG approaches its nominal value, i.e. $v_{o, m a g i} \rightarrow v_{\text {ref }}$. Since the amplitude of the DG output voltage is

$$
v_{o, \text { magi }}=\sqrt{v_{\text {odi }}^{2}+v_{o q i}^{2}}
$$

the synchronization of the voltage amplitude can be achieved by choosing the control input $\mathrm{V}_{\text {ni }}$ such that $v_{\text {odi }} \rightarrow v_{\text {ref }}$. Therefore, for the secondary voltage control, the output and control input are set to $\mathrm{y}_{\mathrm{i}}=\mathrm{v}_{\text {odi }}$ and $\mathrm{u}_{\mathrm{i}}=\mathrm{V}_{\mathrm{ni}}$, respectively.

\subsection{Proposed multi-agent Configuration}

Hierarchical multi-agent control architecture for microgrid system is shown in Fig. 3.14. This multi-agent based configuration includes the power system model, controller agents, the microgrid central controller and the communication links. It illustrates how the measurement signals are transmitted from physical layer to the cyber layer and how the control signals are transmitted among the agents and MGCC through communication links in order to assign the proper control actions for the actuators of each DG. The physical layer has the same configuration as the microgrid case study in section 2 shown in Fig. 3.7. 


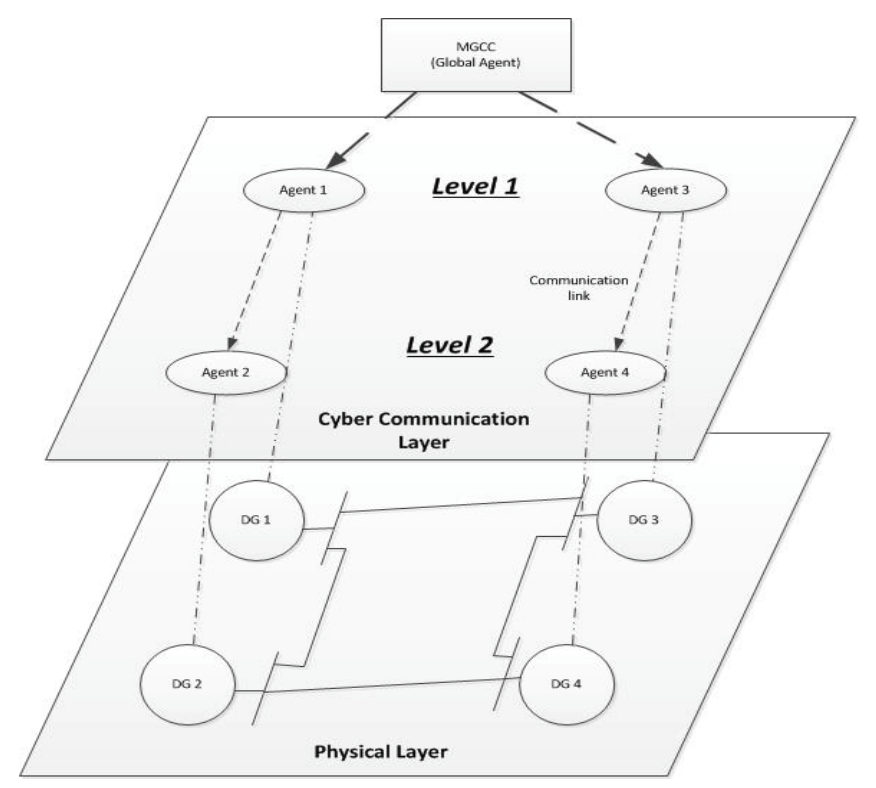

Fig. 3.14. Configuration of the proposed multi agent system for microgrid control

Each generation unit has been assigned with an agent which is communicating with its neighbors and probably the global agent. The global agent contains a central controller unit, which is responsible for coordinating the first level local agents in order to optimize a global cost function. There are two levels of local agent, which are communicating, based on Fig. 3.15 structure. Firstlevel agents are communicating with the MGCC (or global agent) in order to receive the control signal as the reference input of their controller based on Eq. (3.46). Then, the second-level local agents will communicate with those agents with higher level (first-level local agent) to incorporate their measurements in forming the agents' control signals based on Eq. (3.47).

$$
U_{c i}=U_{i}-U_{r e f} \quad i \in 1^{s t} \text { level }
$$

In (3.46) $\mathrm{U}_{\mathrm{ci}}$, and $\mathrm{U}_{\mathrm{i}}$ are the control and measurement signals of the agent $\mathrm{i}$ in the first-level agents, respectively. $\mathrm{U}_{\text {ref }}$ is the reference signal that is provided by global agent. This value could be equal to nominal voltage for voltage control algorithm.

$$
U_{c j}=U_{j}-U_{i} \quad i \in 1^{s t} \text { level \& } j \in 2^{\text {nd }} \text { level }
$$

In (3.47) $\mathrm{U}_{\mathrm{cj}}$, and $\mathrm{U}_{\mathrm{j}}$ are the control and measurement signals of the agent $\mathrm{j}$ in the second-level agents, respectively.

Based on Eq. (3.46) and (3.47), the first-level local agents are communicating with global agent and the second-level local agents are communicating with the first-level local agents in its neighborhood for obtaining its reference signal. This hierarchical structure improves the reliability of the system comparing to the centralized multi-agent method and reduces the burden in the communication links. Also, it is more accurate than decentralized or distributed multi-agent methods since in contrast with decentralized solution, it uses the information of neighbor agents as well as local data. Comparing to the distributed method, it has faster response to the disturbances due to the less number of levels between agents. In fact, there could be $3^{\text {rd }}$ and higher level of agents in distributed solution based on the network configuration.

In this configuration, each local agent is also capable of analyzing the situation and making decision in case of contingencies and loss of communication with the neighbor agent. This configuration in microgrid is called hierarchical multi-agent structure. The fuel cell and DEG are first-level local agents and WTG and PV along with their batteries are second-level local agents in this microgrid.

The proposed hierarchical structure of multi-agent is compared with centralized and distributed multi-agent structure with more details in Fig. 3.15. 
KESHTKAR: MODELING AND CONTROLLING A HYBRID MULTI-AGENT BASED MICROGRID IN PRESENCE OF DIFFERENT PHYSICAL AND CYBER COMPONENTS

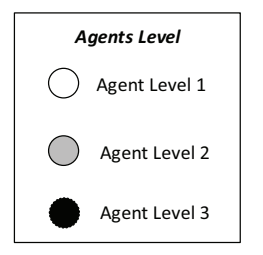

Hierarchal Solution

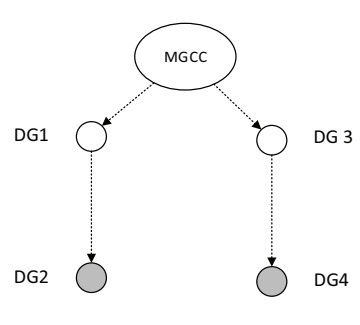

DG1
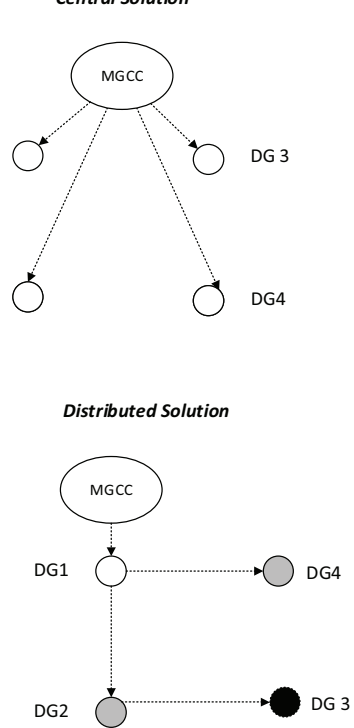

Fig. 3.15. Multi-agent microgrid in different structures.

It can be seen that, reliability of the system decreases when the system is more centralized due to the presence of critical point of failure which is the global agent. Although the complication of the communication system is increased by decentralization, implementation of hierarchal solution is more practical than the centralized one. In centralized solution the entire agents should connect to the global agent, which in large system is not possible and even if it were possible it would be expensive. On the other hand, going towards the distributed strategy, agent's levels increase which means the reference signal will be received by the lowest level agent with more time delay compared to the hierarchal one. This makes a trade-off which results in choosing hierarchal strategy for voltage and frequency control of microgrid. The controller performance using these three structures is discussed in the simulation results section.

\subsection{Control process}

Hierarchical multi-agent based structure proposed in the previous part is used to implement the secondary control of microgrids. The proposed control synchronizes the voltage and frequency of distributed generators (DG) buses to the nominal values and shares the active and reactive power among DGs based on their ratings. This control is implemented through a communication network among the agents, the first-level agents receive the reference value directly by communicating to the global agent and the other DGs only require their own information and the information of its neighbors on the communication network (higher-level neighbor). Due to the hierarchical structure of the communication network, the requirements for a central controller and complex communication network are limited to the first-level local agents, and the system reliability is improved. Simulation results verify the effectiveness of the proposed secondary control for a microgrid test system.

\section{A. Control Algorithm Statement}

Microgrid is considered as a multi-agent system based on the structure in Fig. 3.14, where each DG is an agent. Nonlinear dynamics of the inverter based DGs, presented in section II, are considered for modeling the physical layer of the microgrid. In this section, hierarchical control protocol is derived for each DG that synchronizes the DG voltages and frequencies to the nominal value and allocates the active and reactive power of DGs based on their power ratings. The proposed secondary control is 
implemented through the communication network. Each controller agent receives the measured voltage and frequency signals from the physical layer and minimizes the difference between measured value and their specified reference as defined in Eq. (3.46) and (3.47).

In the cyber layer each DG only requires its own information and the information of its higher-level neighbors, therefore it is more reliable than centralized secondary controls.

\section{B. Control Algorithm Formulation}

The primary control is implemented in the local controller at each DG by the droop technique. Droop technique prescribes a desired relation between the frequency and the active power, and between the voltage amplitude and the reactive power shown in (3.48) and (3.49) (similar to (3.7) and (3.8) for each DG).

$$
\begin{gathered}
\omega_{o i}=\omega_{n i}^{*}-m P_{i} \\
v_{\text {odi }}^{*}=V_{i}^{*}-n Q_{i}
\end{gathered}
$$

In (3.49) $V_{i}^{*}$ and $\omega_{n i}^{*}$ are the primary voltage and frequency control reference and $\mathrm{m}$ and $\mathrm{n}$ are the frequency-active power and voltage-reactive power droop coefficient.

The secondary frequency control chooses $\omega_{n i}$ such that the angular frequency of each DG synchronizes to its nominal value, i.e. $\omega_{i} \rightarrow \omega_{r e f}$. It should be noted that once the secondary frequency control is applied, the DG output powers are allocated according to the same pattern used for primary control [31]-[32]. After applying the primary control, the DG output powers satisfy the following equality

$$
\begin{aligned}
& m_{P 1} P_{1}=\ldots=m_{P N} P_{N} \\
& n_{Q 1} Q_{1}=\ldots=n_{Q N} Q_{N}
\end{aligned}
$$

Since the droop coefficients $\mathrm{m}$ and $\mathrm{n}$ are chosen based on the power rating of $\mathrm{DGs}, \mathrm{P}_{\operatorname{maxi}}$ and $\mathrm{Q}_{\operatorname{maxi}}$, above equations are equivalent to

$$
\begin{gathered}
\frac{P_{1}}{P_{\max 1}}=\ldots=\frac{P_{N}}{P_{\max N}} \\
\frac{Q_{1}}{Q_{\max 1}}=\ldots=\frac{Q_{N}}{Q_{\max N}}
\end{gathered}
$$

Therefore, the secondary frequency control must also satisfy above equations. For the secondary frequency control, the outputs and inputs are $y_{i}=\omega_{i} \&$ vi and $u_{i}=\omega_{n i}^{*} \& V_{i}^{*}$, respectively.

Conventionally, the secondary control is implemented by using a centralized controller for the whole microgrid having the proportional-plus-integral (PI) structure [28]. In a centralized control structure, the central controller communicates with all DGs in the microgrid through a star communication network. A centralized control structure deteriorates the system reliability [31]. Hence, the hierarchical multi-agent structure is proposed for implementing the optimal secondary control of the microgrid.

The control parameters are optimized by Particle Swarm Optimization (PSO). PSO is a multi-agent search approach, which traces its evolution to the motion of a flock of birds searching for food. It uses a number of particles that are called a swarm. Each particle traverses the search space searching for the global minimum (or maximum). In a PSO system, particles fly within a multidimensional search space. During flight, each particle sets its position based on its own experience and the experience of neighboring particles. Hence, it makes use of the best position encountered by itself and its neighbors. Similarly, the swarm 
KESHTKAR: MODELING AND CONTROLLING A HYBRID MULTI-AGENT BASED MICROGRID IN PRESENCE OF DIFFERENT PHYSICAL AND CYBER COMPONENTS

direction and speed of a particle is determined by the history experience obtained by itself as well as a set of its neighboring particles [33]. The objective function which is minimized by using PSO in this chapter is formulated as $J=U_{i}-U^{*}$. $\mathrm{U}_{\mathrm{i}}$ is the voltage (frequency) signal and $\mathrm{U}^{*}$ is the reference voltage (frequency) of each DG based on (3.46) and (3.47) for voltage (frequency) control system. Further descriptions about the implementation of PSO in the modeled microgrid can be found in [34].

\section{Dual Heuristic Dynamic Programming (DHP)}

Another proposed approach which is utilized to replace PI controller is DHP which classified in ADP family. This algorithm was described in part 2.3 of chapter 2 and is reformulated in this section. ADP is a type of Adaptive Critic Design (ACD) technique which consists of three neural networks: model action, and critic networks. The model network can identify the time-varying dynamics of the nonlinear power system. The critic network, which approximates the control objective, trains the nonlinear action network to create an optimal control mapping between the plant feedbacks and control actions [35]. In this chapter, the objective function of the control methods is the deviation of frequency and voltage due to load and wind power variations.

\section{C.1 Recurrent Neural Networks for Adaptive Critic Designs (ACDs)}

In this chapter, the Dual Heuristic dynamic Programming (DHP), which is a technique in the ACD family, is used for the frequency and voltage control purpose. Fig. 3.16 shows the schematic diagram of DHP where the critic network directly estimates $\lambda(\mathrm{k})$, the gradient of $\mathrm{J}(\mathrm{k})$ (the cost-to-go control objective) with respect to the plant outputs.

It should be noticed that in all materials of this part, $\mathrm{u}(\mathrm{k})$ as input of the power plant is the power deviation of the controllable sources $\left(\Delta P_{c} \& \Delta Q_{c}\right), \mathrm{y}(\mathrm{k})$ is the frequency and voltage deviation ( $\left.\Delta f \& \Delta V\right)$ as the output of the power system model, and $\mathrm{J}(\mathrm{k})$ is the control error signal which is the difference between the frequency and voltage signal and their reference value based on (3.46) and (3.47).

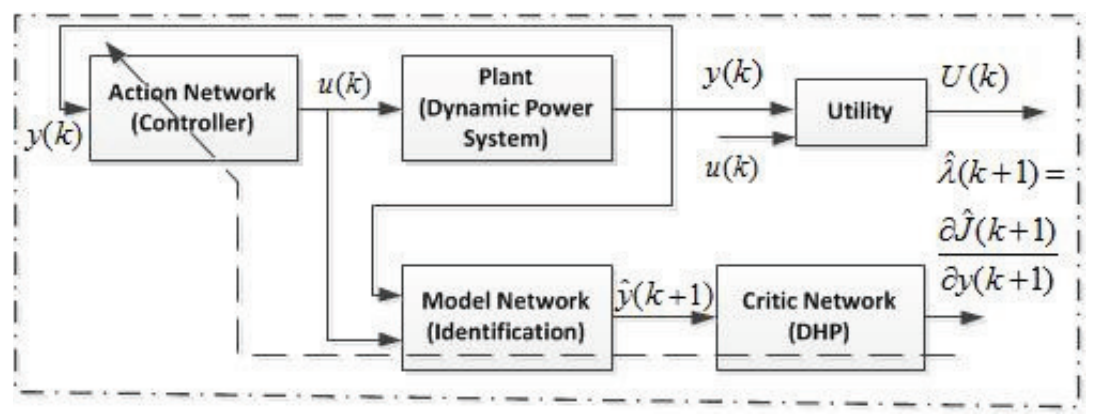

Fig. 3.16. Schematic diagram of DHP neurocontrol design with RNNs

\section{C.2 Training of a DHP-based Neurocontroller using Recurrent Neural Networks (RNNs)}

The training of a DHP-based neurocontroller is done in two steps in this chapter: 1) offline training of the model network; and 2) online training of all three networks [36]. In the following section, $W_{\mathrm{im}}, \mathrm{W}_{\mathrm{sm}}$, and $\mathrm{W}_{\text {om }}$ are weights for the model network, $\mathrm{W}_{\mathrm{ic}}, \mathrm{W}_{\mathrm{sc}}$, and $\mathrm{W}_{\mathrm{oc}}$ are weights for the critic network, $\mathrm{W}_{\mathrm{ia}}, \mathrm{W}_{\mathrm{sa}}$, and $\mathrm{W}_{\mathrm{oa}}$ are weights for the action network.

\section{C.3 Offline Training of Model Network}

The plant is first operated in an open-loop action network. Random signals are injected into the plant inputs, $\mathrm{u}(\mathrm{k})$, over a wide operating range. The plant responses, $\mathrm{y}(\mathrm{k})$, are recorded along with $\mathrm{u}(\mathrm{k})$. Having values of the recorded $\mathrm{u}(\mathrm{k})$ and $\mathrm{y}(\mathrm{k})$ at different operating points, the model network is trained offline to minimize the following mean squared error (MSE): 


$$
\begin{aligned}
& E_{m}(k)=\left\|e_{m}(k)\right\|^{2}=\|y(k)-\hat{y}(k)\|^{2} \\
& M S E=\text { average }\left[\sum_{\substack{\text { operating } \\
\text { points }}} \sum_{k} E_{m}(k)\right]
\end{aligned}
$$

In $(3.54) \hat{y}(k)$ is the one-step delay of the model network output. In other words, the model network is trained to identify the plant dynamics.

The model network is initialized with small random weights. During the training step, the one-step model prediction error and error gradients are obtained as follows,

$$
\begin{aligned}
& \frac{\partial E(k)}{\partial W_{o}}=[\nabla g] \cdot \frac{\partial E(k)}{\partial o(k)} \cdot s(k)^{\prime} \\
& \frac{\partial E(k)}{\partial W_{s}}=[\nabla f] W_{o}^{\prime} \cdot[\nabla g] \cdot \frac{\partial E(k)}{\partial o(k)} \cdot s(k-1)^{\prime} \\
& \frac{\partial E(k)}{\partial W_{i}}=[\nabla f] W_{o}^{\prime} \cdot[\nabla g] \cdot \frac{\partial E(k)}{\partial o(k)} \cdot i(k)^{\prime}
\end{aligned}
$$

In (3.56) $\mathrm{i}(\mathrm{k})$ is the input vector, o(k) is the output vector, $\mathrm{s}(\mathrm{k})$ is the $\mathrm{RNN}$ internal state vector, $f(\cdot)$ and $g(\cdot)$ are activation functions. $\mathrm{E}(\mathrm{k})$ is an error measurement related the RNN outputs. More details on calculating this error for the model, critic and action networks are described in the rest of this section. $[\nabla g]$ and $[\nabla f]$ are diagonal matrices containing the gradients of $g(\cdot)$ and $f(\cdot)$, respectively.

The model weights are then updated based on the (3.57). The model training continues until the MSE calculated in the testing step drops below a specific value.

$$
\begin{aligned}
& W_{o}(k+1)=W_{o}(k)-r_{o} \frac{\partial E(k)}{W_{o}} \\
& W_{s}(k+1)=W_{s}(k)-r_{s} \frac{\partial E(k)}{W_{s}} \\
& W_{i}(k+1)=W_{i}(k)-r_{i} \frac{\partial E(k)}{W_{i}}
\end{aligned}
$$

\section{C.4 Online Training of Model, Critic, and Action Networks}

After the offline training, the model network is used to provide system-wide cross-coupling sensitivity signals, $\partial \mathrm{y}(\mathrm{k}+1) / \partial \mathrm{u}(\mathrm{k})$, over a wide operating range. When the critic and action networks are trained, the model weights are continuously updated with a small learning rate to ensure tracking of new operating conditions.

An RNN DHP critic network is trained online to approximate $\lambda(\mathrm{k}+1)$, the partial derivative of $\mathrm{J}(\mathrm{k}+1)$ with respect to $\mathrm{y}(\mathrm{k}+1)$, by minimizing the error in (3.58).

$$
\begin{aligned}
E_{c}(k)= & \left\|e_{c}(k)\right\|^{2} \\
e_{c}(k)= & \hat{\lambda}(k)-\left\{\frac{\partial U(k)}{\partial y(k)}+\left[\frac{\partial u(k)}{\partial y(k)}\right] \frac{\partial U(k)}{\partial u(k)}\right. \\
& \left.+\gamma\left[\frac{\partial \hat{y}(k+1)}{\partial y(k)}+\frac{\partial \hat{y}(k+1)}{\partial u(k)} \frac{\partial u(k)}{\partial y(k)}\right] \hat{\lambda}(k+1)\right\}
\end{aligned}
$$

The training of the critic network is done online along with the action network in the loop. The training starts with a small discount factor $\gamma$. As the critic weights converge, the discount factor is gradually increased.

The optimal control objective of the controller is to minimize $\mathrm{J}(\mathrm{k})$ at every time step $\mathrm{k}$, which is accomplished by training an RNN action network. The action network is trained to approximate the optimal control law by minimizing (3.59). 
KESHTKAR: MODELING AND CONTROLLING A HYBRID MULTI-AGENT BASED MICROGRID IN PRESENCE OF DIFFERENT PHYSICAL AND CYBER COMPONENTS

$$
\begin{aligned}
& E_{a}(k)=\left\|\frac{\partial \hat{J}(k)}{\partial u(k)}\right\|^{2} \\
& \frac{\partial \hat{J}(k)}{\partial u(k)}=\frac{\partial U(k)}{\partial u(k)}+\gamma\left[\frac{\partial \hat{y}(k+1)}{\partial u(k)}\right] \hat{\lambda}(k+1)
\end{aligned}
$$

During the training, when $\mathrm{E}_{\mathrm{a}}(\mathrm{k})$ becomes zero, $\mathrm{u}(\mathrm{k})$ is the optimal control action that minimizes $\mathrm{J}(\mathrm{k})$ in the local region. Global optimal is obtained by exposing the RNN action network to different system conditions.

Assuming a linear output activation function for the critic network, the critic network output at time k is calculated by

$$
\hat{\lambda}(k+1)=W_{o c} S_{c}(k)=W_{o c} f_{c}\left[W_{i c} \hat{y}(k+1)+W_{s c} S_{c}(k-1)\right]
$$

where $s_{c}(k)$ is the internal state vector of the critic network, and $f_{c}(\cdot)$ is the hidden layer activation function of the critic network. With a truncated depth of one, the action error (which is obtained by (3.59)) gradients with respect to its weights can be obtained using (3.56). During each sampling step, the weights of both the action and critic networks are updated based on (3.57). The online training continues until both the critic and action errors converge to zero.

\section{IV- Simulation Results}

Implementation of proposed secondary control and multi-agent based control algorithm are tested and analyzed in this section.

\subsection{Proposed secondary control}

The inverter-based microgrid is modeled based on the nonlinear equations described in section III. The stability of the linearized model of this microgrid was tested in section II. Implementation of multi-agent control algorithm is tested in this section.

Fig. 3.17 shows the frequency response of each DG after a load change at $\mathrm{t}=0$ and $1 \mathrm{~s}$ using the proposed secondary control. It can be seen that the frequency deviation is damped very quickly and the steady state error is also eliminated. In these results PSO algorithm is used for tuning the control parameters of each agent to minimize the frequency deviations. It also can be seen that using PSO, the frequency deviation is alleviated appropriately.

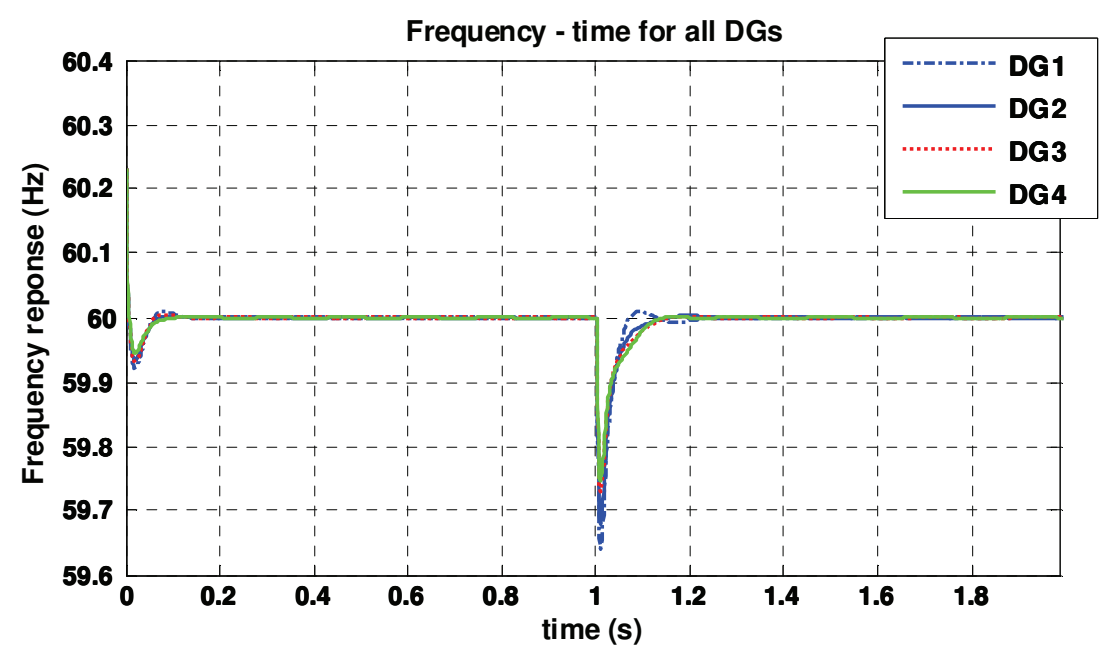

Fig. 3.17. Frequency response of the microgrid using proposed secondary control 


\subsection{Proposed multi-agent control}

The PSO algorithm is used for optimizing the controller actions in all the case studies in order to compare the effect of multiagent structure on the responses. First of all, centralized configuration for the proposed multi-agent control system is employed and the results of frequency, current, and voltage of each DG due to a sudden load change at 0s and 360s are shown in Fig. 3.18. The results show that the proposed secondary controllers are able to damp the frequency oscillations and voltage error in the centralized multi-agent system.

Voltage variations after using centralized, hierarchical and distributed multi-agent system for the same perturbation in the microgrid are shown in Fig. 3.19. The results show the reference control signal of each agent is provided by its neighbor agent at the higher level. For example, in distributed solution shown in Fig. 3.19, DG3 which is at the third level from the global agent has the slowest response to the frequency oscillations which results in higher overshoot and settling time values. The reason behind that is the delay in receiving the reference signal which is transmitted from higher level (DG1 and DG2) to DG3. In the proposed hierarchical solution in Fig. 3.19, it can be seen that the voltage responses of second level agents are following the signals of first level agents that creates a small delay in the settling time. However, the system is stabilized in proper time and the reliability of the system is increased with this new structure.

For comparison purposes, voltage responses of all DGs using different multi-agent structures are shown together in Fig. 3.20 to Fig. 3.22. It can be seen that the agents in the same level act similarly in different structures of multi-agent. However, the lower level agents in distributed and hierarchical structures, act a little slower. That drawback is compensated through being more practical in implementation, higher reliability, and less volume of communication burden. In overall, by considering the advantages, the proposed hierarchical multi-agent perform better than the other two methods.

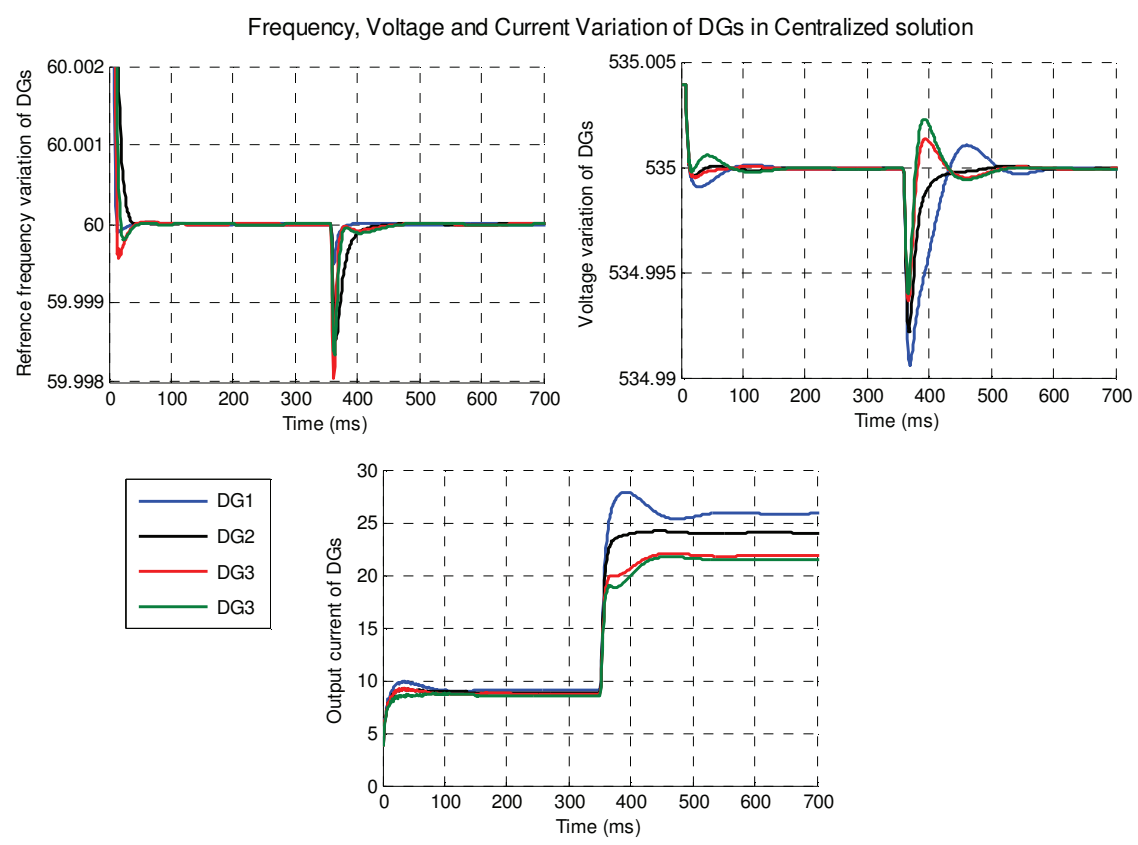

Fig. 3.18. Frequency, voltage, and current response of the centralized multi-agent based microgrid 


\section{KESHTKAR: MODELING AND CONTROLLING A HYBRID MULTI-AGENT BASED MICROGRID IN PRESENCE OF DIFFERENT PHYSICAL AND CYBER COMPONENTS}
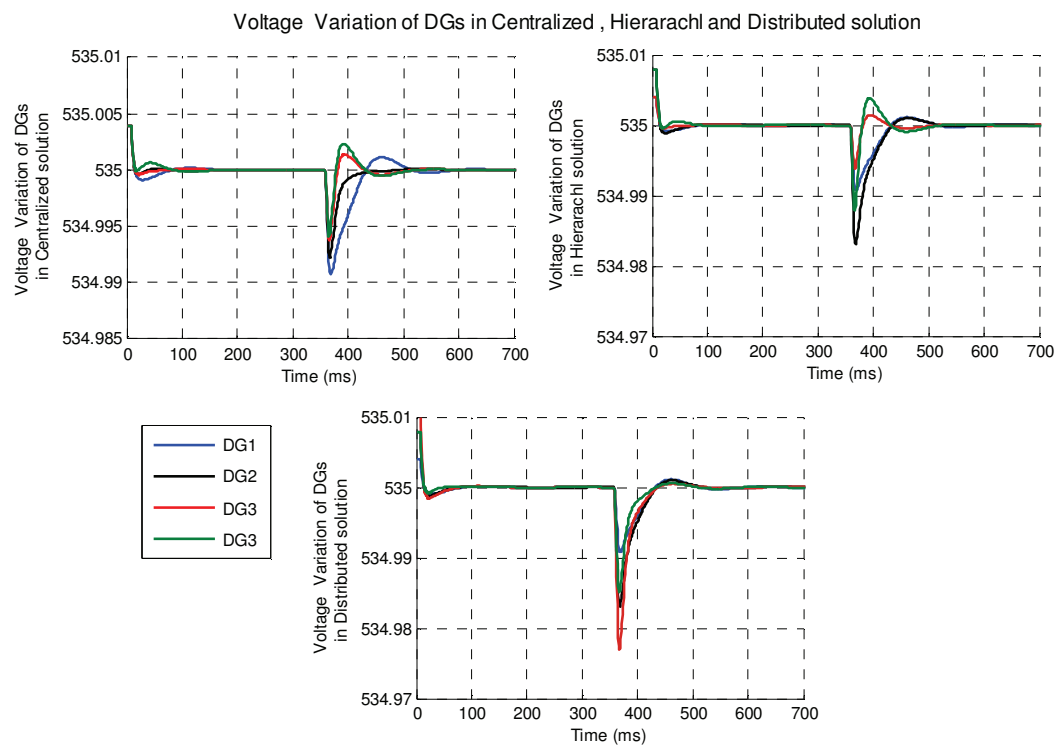

Fig. 3.19. Voltage response of the centralized, hierarchical, and distributed multi-agent based microgrid

One of the purposes of this chapter is learning about the effect of agent's level on the voltage response to the control set-point. Agent level can be defined based on the number of nodes that is used to pass the control data to a specific agent. In fact in centralized control all the agents can be considered as agent level one because all of them are in communication with the central controller directly to receive the control reference set-point. The level of agents in centralized solution along with the other two solutions namely, hierarchal and distributed, can be seen in Fig. 3.15. In hierarchal solution there are 2 levels of agents which means only a part of agents are receiving voltage set-point directly, and the remaining part are communicating with first level agents. In distributed solution only one agent is receiving the voltage set-point from control center and it would pass it through other agents until all of them receive this set-point. This solution obviously has more than 2 levels of agents which are shown in the Fig. 3.15.
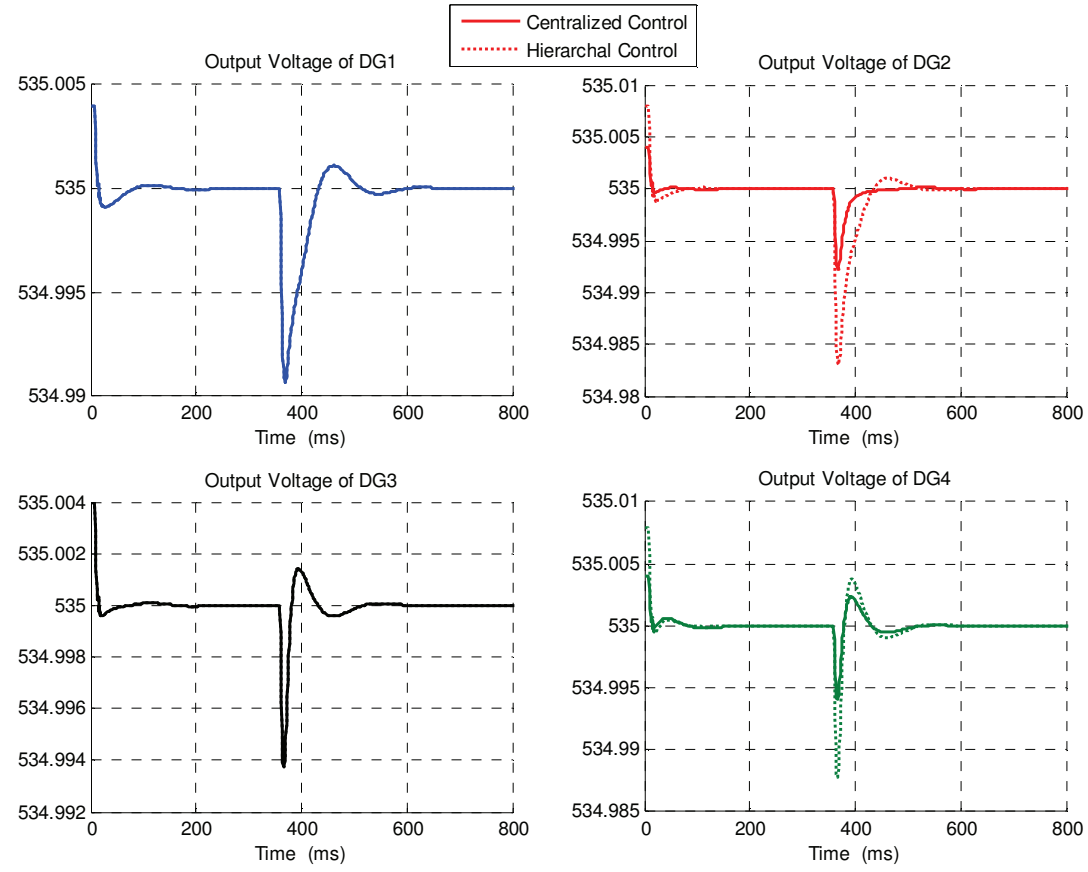

Fig. 3.20 Voltage response of DGs using centralized and hierarchical multi-agent based control 

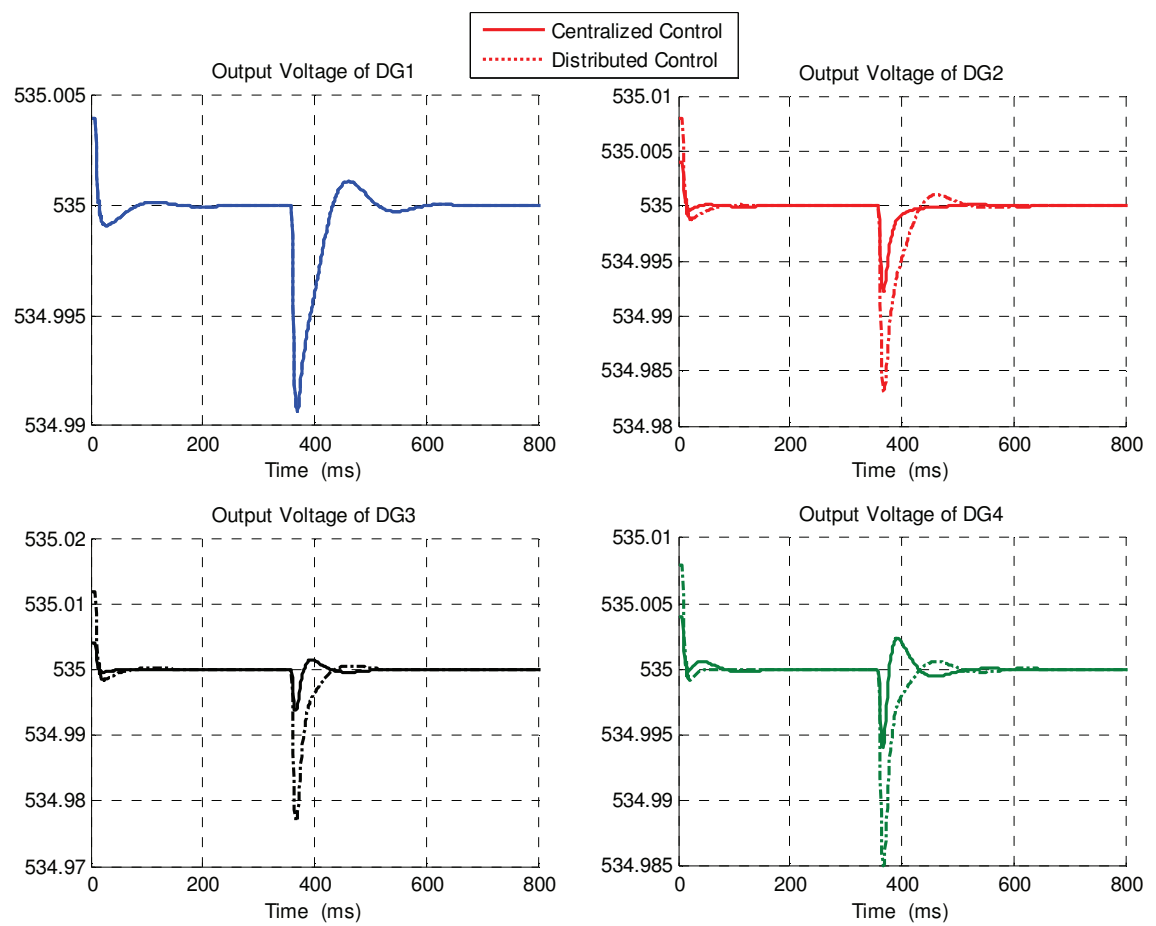

Fig. 3.21 Voltage response of DGs using centralized and distributed multi-agent based control

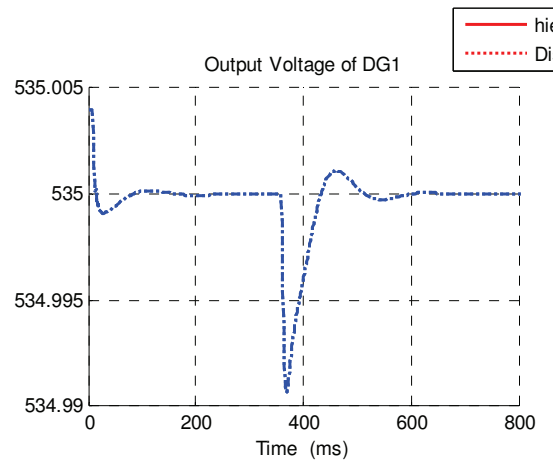

hierarchal Control
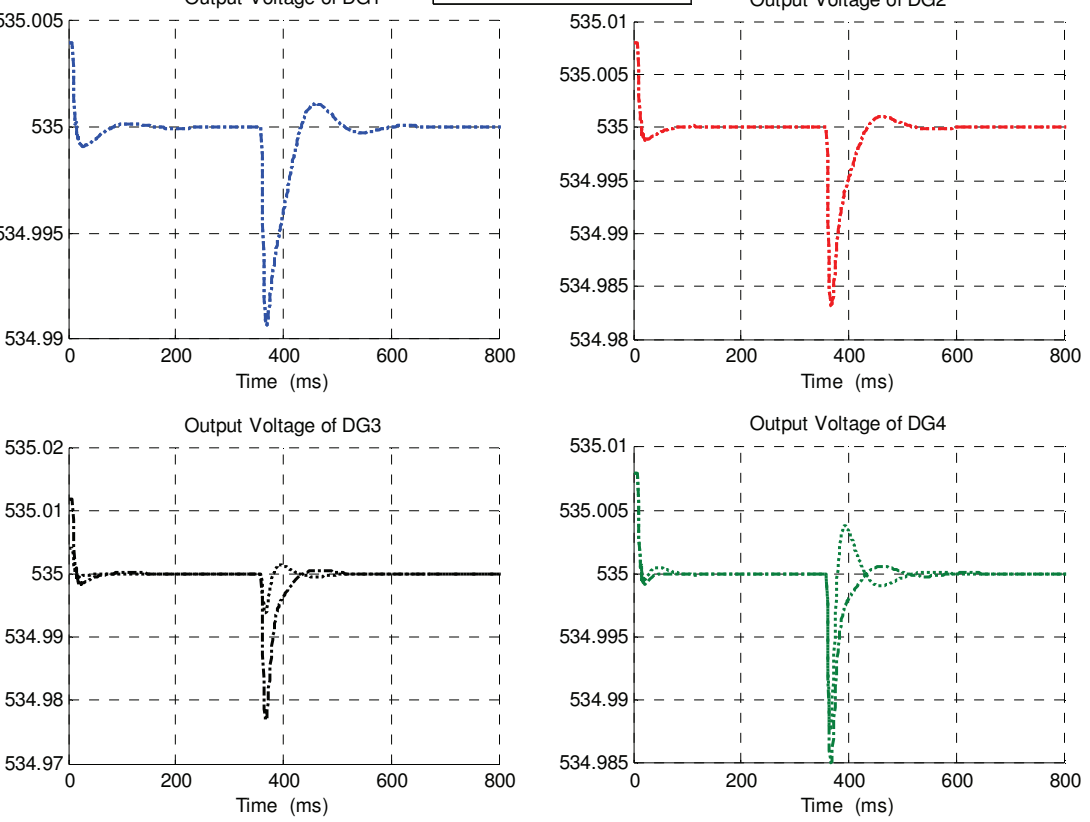

Fig. 3.22. Voltage response of DGs using hierarchical and distributed multi-agent based control

\subsection{Analysis on Stochastic Disturbance}

As it was discussed in chapter 2, there are some uncertainties related to wind, PV, and load in real microgrid power system that need to be taken into account. In previous simulation results, normal load changes were applied to the modeled microgrid in order to analyze the controller performance. In this part, stochastic load disturbances are applied to the nonlinear model of the system with hierarchical multi-agent controller in order to analyze the effect of stochasticity on the performance of the hierarchal secondary control. To create the load uncertainty stochastically, the model is obtained from part 2 of chapter 2 . The voltage response of the system are presented in Fig. 3. 23 and Fig. 3. 24. 


\section{KESHTKAR: MODELING AND CONTROLLING A HYBRID MULTI-AGENT BASED MICROGRID IN PRESENCE OF DIFFERENT PHYSICAL AND CYBER COMPONENTS}

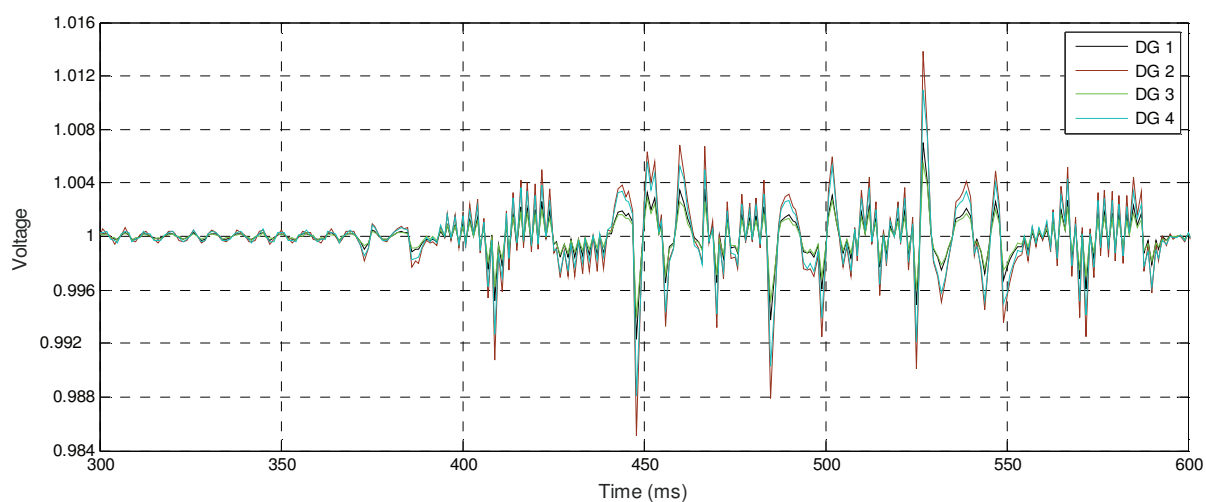

Fig. 3.23. Voltage response of the hierarchical multi-agent based microgrid with stochastic load

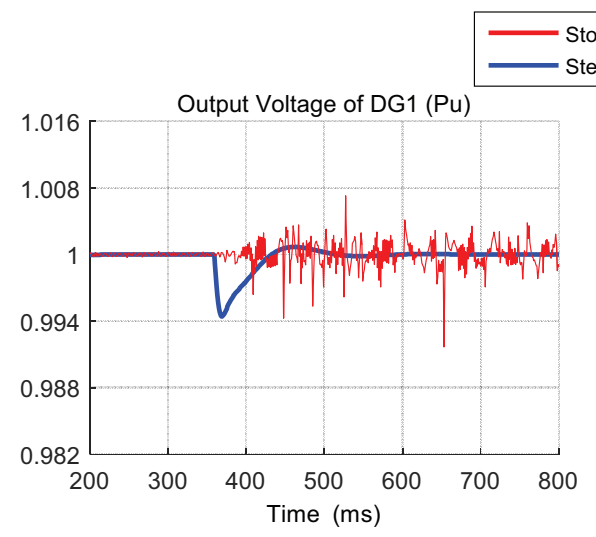
Stochastic Load at $350 \mathrm{~ms}$
Step Load change at $350 \mathrm{~ms}$
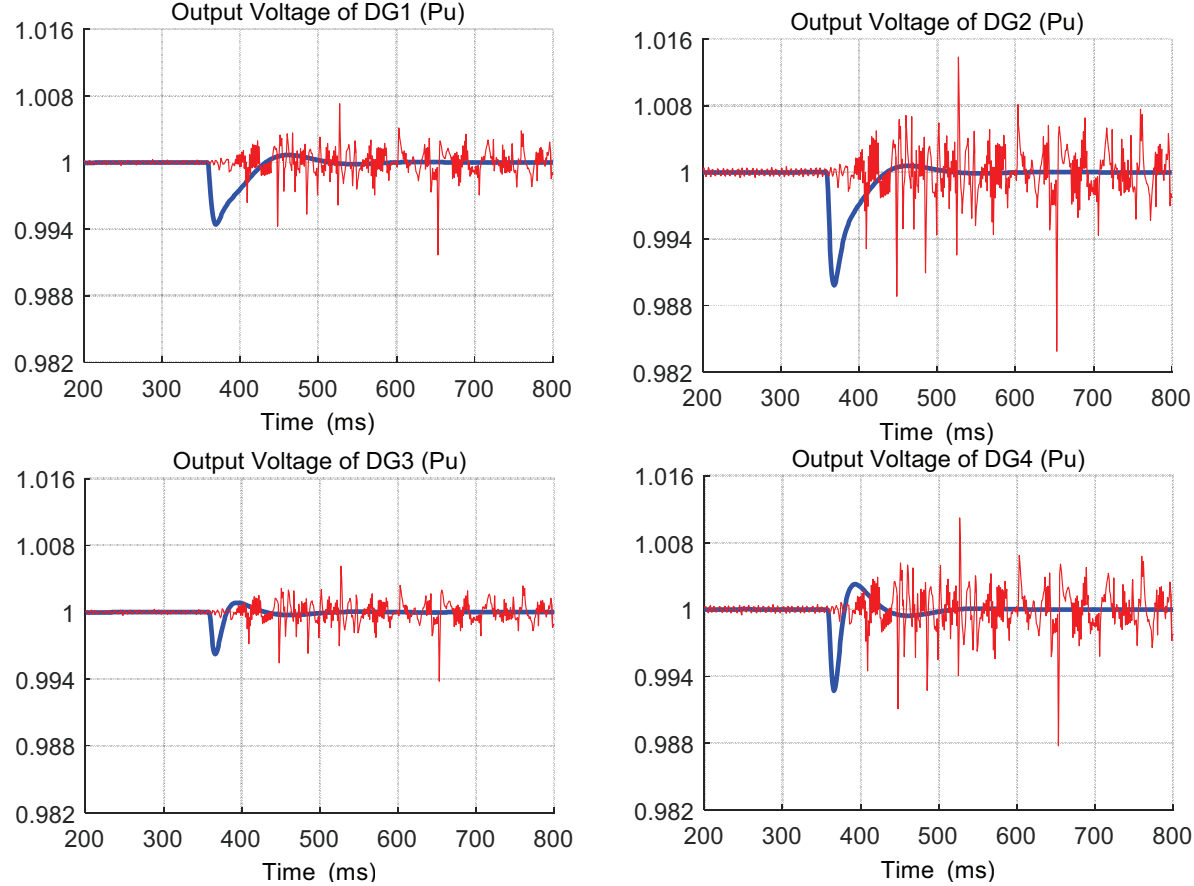

Fig. 3.24. Voltage response of the hierarchical multi-agent based microgrid with normal and stochastic load change

Fig. 3. 23 shows the voltage response of the system during the stochastic load changes applied to the system. In spite of large voltage disturbances due to stochastic load, the cooperative hierarchal controllers are able to decrease the overshoots value and stabilize the voltage deviations. Fig. 3. 24 compares the voltage of the DGs in presence of normal load change and stochastic disturbances. The stochastic and normal load perturbations starts at $\mathrm{t}=350 \mathrm{~ms}$ and it causes the voltage of the system to deviate from nominal value. It is observed that the controller acts at the time of deviations where the global agent coordinates and provide the reference control input signal to other local agent controllers to damp the oscillations within stability margin. Also, It can be seen that the stochastic behavior of the load causes more deviations in the output signals of the system comparing to normal load change, however the proposed control method is able to damp this kind of oscillations and the system remains stable. 


\subsection{Distribution Line Mutual Coupling}

Three-phase unbalance modeling in a practical distribution feeder is different because of the mutual coupling between phase conductors and the grounded neutral line due to load currents of the distribution feeder. This part will investigate the effect of mutual coupling between phase conductors on the simulation results. MATLAB three-phase mutual inductance toolbox is used for modeling mutual coupling of all the lines in the microgrid model. The parameters of mutual coupling are shown in Table 3.I. Considering mutual coupling in distribution line model of the network and reproducing the results using the hierarchical multiagent structure of microgrid, frequency and voltage outputs are compared with the case using existing network model presented in section II of this chapter.

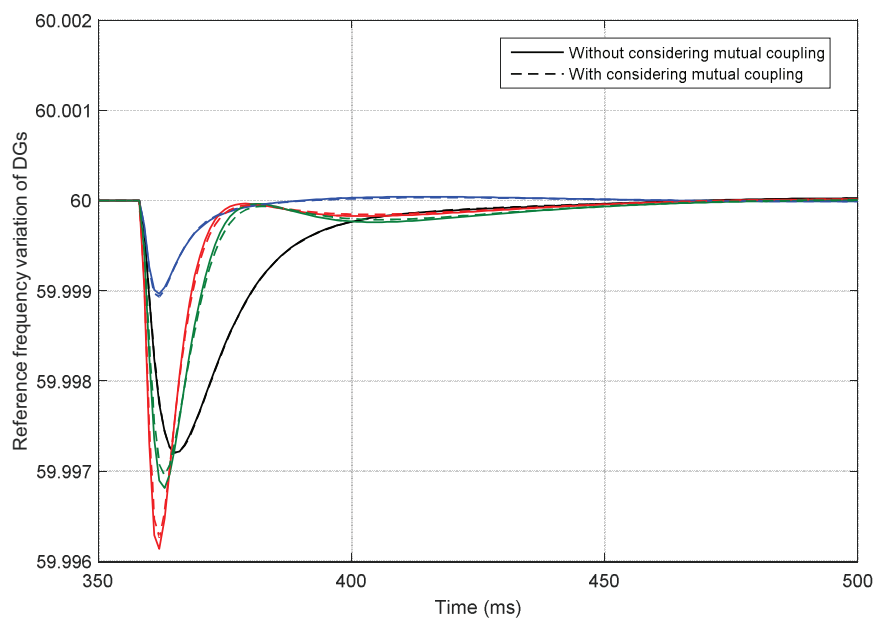

Fig. 3.25. Frequency response of the hierarchical multi-agent based microgrid with and without line mutual coupling

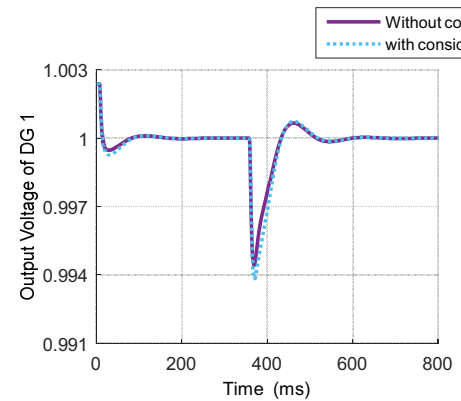

With considering mutua
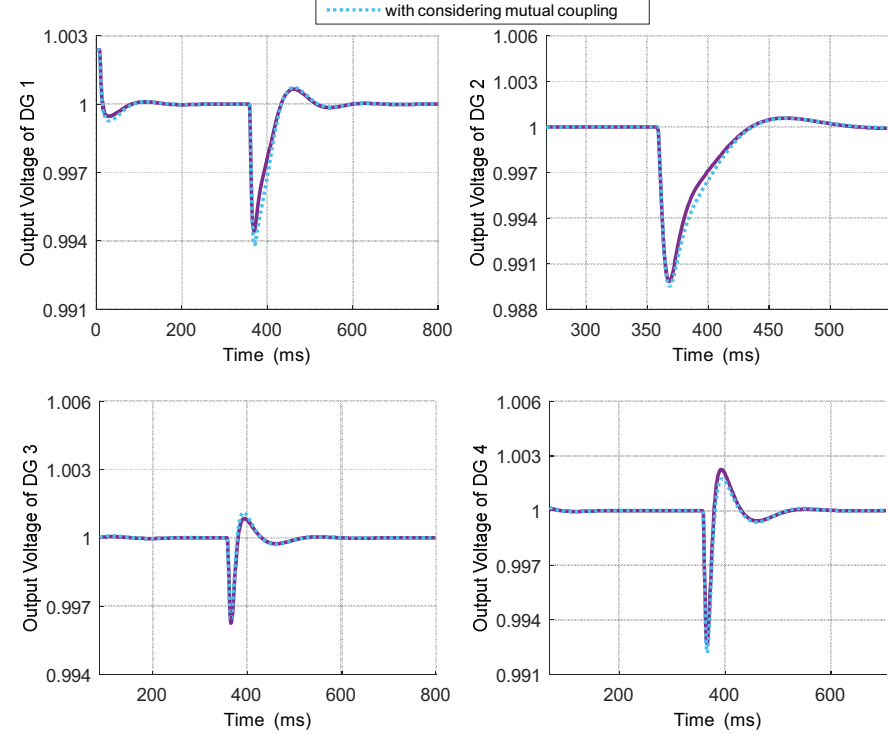

Fig. 3.26. Voltage response of the hierarchical multi-agent based microgrid with and without line mutual coupling

Table 3.I. parameters of mutual coupling

\begin{tabular}{|c|c|c|c|}
\hline \multicolumn{2}{|c|}{ Positive sequence parameters } & \multicolumn{2}{|c|}{ Zero sequence parameters } \\
\hline $\mathrm{R}_{1}(\mathrm{Ohms})$ & $\mathrm{L}_{1}(\mathrm{H})$ & $\mathrm{R}_{0}(\mathrm{Ohms})$ & $\mathrm{L}_{0}(\mathrm{H})$ \\
\hline 0.4 & 0.001 & 0.8 & 0.002 \\
\hline
\end{tabular}


KESHTKAR: MODELING AND CONTROLLING A HYBRID MULTI-AGENT BASED MICROGRID IN PRESENCE OF DIFFERENT PHYSICAL AND CYBER COMPONENTS

Fig. 3. 25 and Fig. 3. 26 present the changes in the frequency and voltage response due to considering the mutual coupling of line model. The line models are updated with new modeling consist of mutual coupling effect and the voltage results due to a load change are compared to the case without mutual coupling model. Dotted curves show the results of integrating mutual coupling in the line model and solid curves represent the results without mutual coupling effect. The results confirms sufficient accuracy of the existing network model in representing the aspects of distribution line.

\subsection{Evaluating the results with existing published method}

In this part, the contribution of this section is evaluated through different simulations using the existing published literatures in this area. Firstly, the same microgrid configuration in paper [38] is used in order to evaluate the correctness of simulation results related to this part. Then, different scenarios have been utilized to compare the proposed multi-agent structure and control algorithm with paper [38] by the same authors as in [38].

\section{A. Evaluation}

In order to evaluate the simulation of this section with [38] two sets of results are compared in Fig. 3.27 and Fig. 3.28. Microgrid configuration and parameters as well as primary control algorithm are applied to the system based on [38]. Fig. 3.27 shows the response of applying primary voltage control on the model microgrid system while the disturbance is applied to microgird at similar severity as in [38]. In the second scenario the same disturbance is applied to microgird, while the secondary control is active. Fig. 3.28 shows the DG terminal voltage amplitudes when the reference voltage value is set to 1 , 0.95 , and 1.05 p.u., respectively.

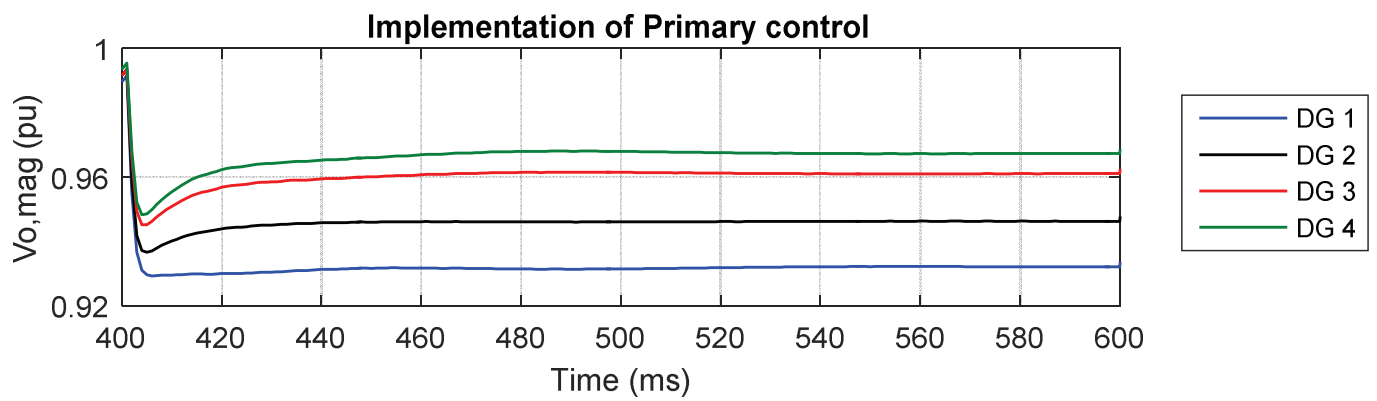

Fig. 3.27. DG output voltages magnitude applying primary control only.

Based on the results of the first scenario in Fig. 3.27, DG terminal voltages obtained from primary control matches the results presented in Fig. 9 of [38] and confirms the performance of the primary control of this section. It can be seen from Fig. 3.28 that the results of applying secondary control in these two literatures are very similar (see Fig. 8 in [38]). The small difference is reasonable due to the different secondary loop used in [38] for voltage control purpose. After evaluating the proposed modeling and method of this section through simulation results, the rest of this part discusses about the contribution of this section on multiagent structure. This analysis is carried out through discussion on the differences between the proposed method in this dissertation and the one in [39]. 

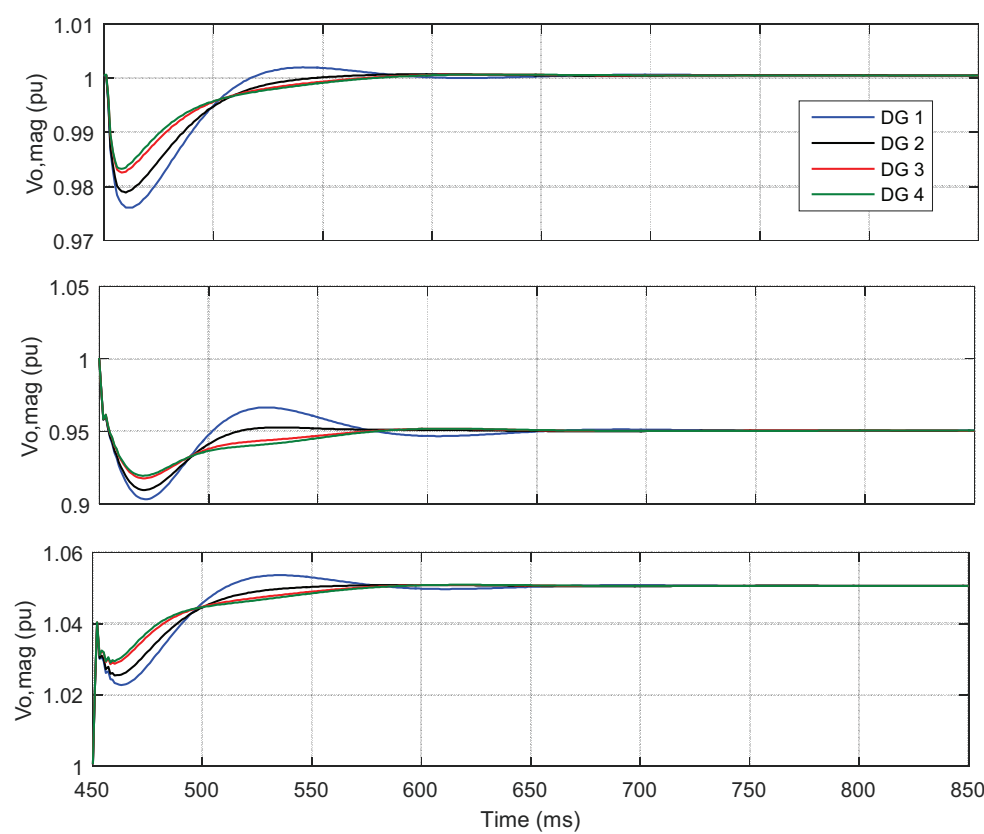

Fig. 3.28. DG output voltages magnitude applying secondary control for (a) $\mathrm{v}_{\text {ref }}=1 \mathrm{pu}$, (b) $\mathrm{v}_{\text {ref }}=0.95 \mathrm{pu}$, and (c) $\mathrm{v}_{\text {ref }}=1.05 \mathrm{pu}$.

\section{B. Contribution comparison}

In order to discuss the contribution of this chapter compared to available literature, the results of applying existing published method in [39] is compared with the proposed strategy. The simulation results include comparison of different scenarios including outage of a DG for analyzing the robustness and control performance of the system in extreme cases.

Bidram et.al. in [39] introduces a distributed cooperative control of multi agent systems inside the secondary voltage control of microgrids. By considering DGs as agents in a microgrid, it represents the secondary control of microgrids as a tracking synchronization problem, where all DGs try to synchronize their terminal voltage amplitude to a pre-specified reference value. For implementation of this control algorithm a distributed multi-agent structure is used in which each DG needs to communicate only with its neighbors. In order to apply the secondary control, the algorithm should choose an appropriate value for the control input (reference voltage). Therefore, the author has added another term to the conventional control input signal so the reactive power sharing as well as voltage would be considered in calculation of control error signal. Fig. 3. 29 shows the implemented secondary voltage control in [39]. The error formula used for the controllers of this dissertation and [39] are also shown in (3.61) and (3.62), respectively.

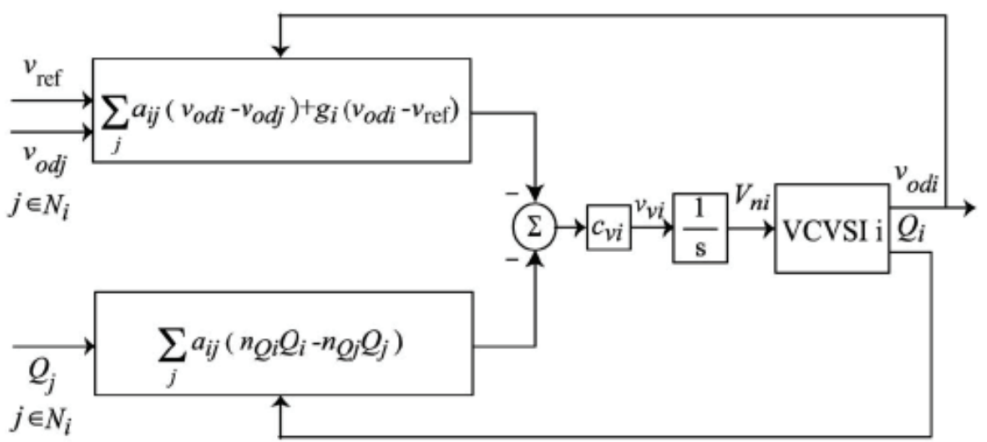

Fig. 3. 29. Block diagram of the distributed secondary voltage control of VSI inverter in [38]

$$
e_{i}=\sum_{j \in N_{i}} a_{i j}\left(v_{o d i}-v_{o d j}\right)+g_{i}\left(v_{o d i}-v_{r e f}\right)
$$


KESHTKAR: MODELING AND CONTROLLING A HYBRID MULTI-AGENT BASED MICROGRID IN PRESENCE OF DIFFERENT PHYSICAL AND CYBER COMPONENTS

$$
e_{i}=\sum_{j \in N_{i}} a_{i j}\left(v_{\text {odi }}-v_{\text {odj }}\right)+g_{i}\left(v_{\text {odi }}-v_{\text {ref }}\right)+\sum_{j \in N_{i}} a_{i j}\left(n_{Q_{i}} Q_{i}-n_{Q_{j}} Q_{j}\right)
$$

In these equations $\mathrm{g}_{\mathrm{i}}$ and $\mathrm{a}_{\mathrm{ij}}$ are the components of communication graph matrix which equal to 1 when there is a connection between node $\mathrm{i}$ and $\mathrm{j}$ and 0 when node $\mathrm{i}$ and $\mathrm{j}$ are not connected. $\mathrm{N}_{\mathrm{i}}$ represent the set of neighbors for node $\mathrm{i}$. Controllers in both cases are implemented through communication network with one-way communication link. These two methods are applied in the modeled microgrid for different scenarios and the results are compared in the following parts.

\section{B.1. DG outage}

In the first set of results an extreme change in the system which is DG outage due to an internal fault has been modeled to compare the robustness of the proposed and [39] methods. In Fig. 3.30, DG bus voltage and frequency responses of the proposed method are presented when DG 4 is removed from the system at $\mathrm{t}=350 \mathrm{~ms}$. The significance of voltage variation after this large change can be observed from these results. However, the proposed method is able to compensate the voltage drop caused by DG 4's outage. Fig. 3.31 compares the performance and robustness of the proposed and [39] methods. It confirms the robustness of both control algorithms in case of large changes such as DG outage. However, since the multi-agent structure in paper [39] is distributed, the time delay existed in transferring the reference control signal from global agent to the local DGs makes the node voltage responses of the further DGs more oscillatory comparing to the responses of proposed hierarchical control method. For instance, the average overshoot in the responses of proposed method is decreased by 5\% comparing with [39]. Therefore, the importance of employing appropriate structure for multi-agent is investigated in this part.
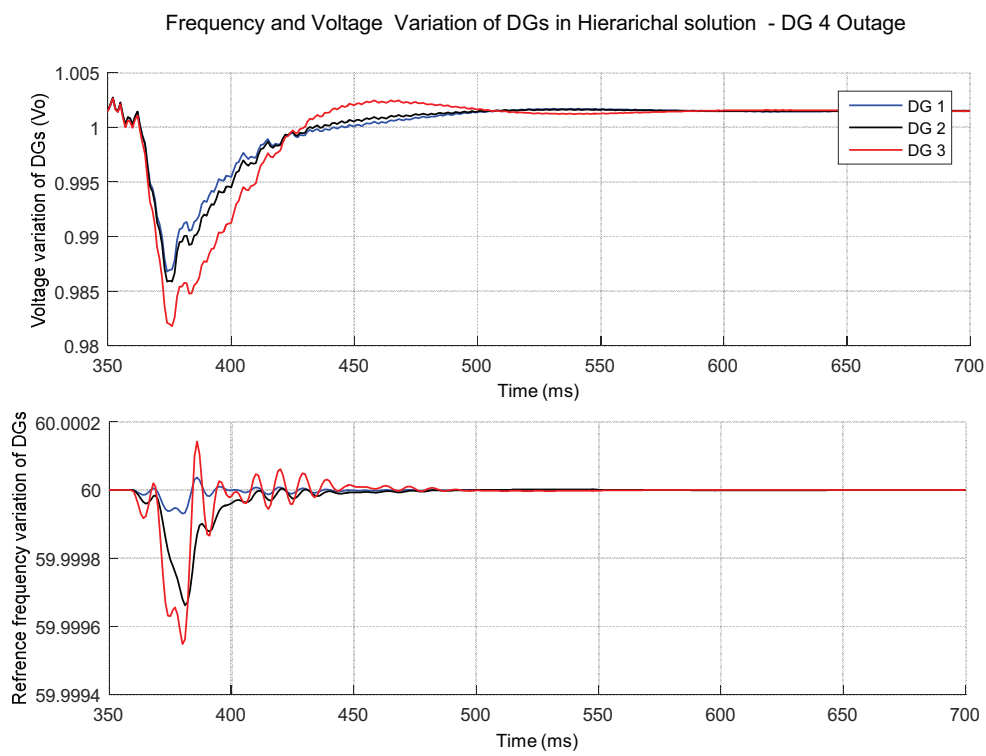

Fig. 3. 30. DGs Voltage and frequency responses of the hierarchical multi-agent based microgrid in case of DG outage 

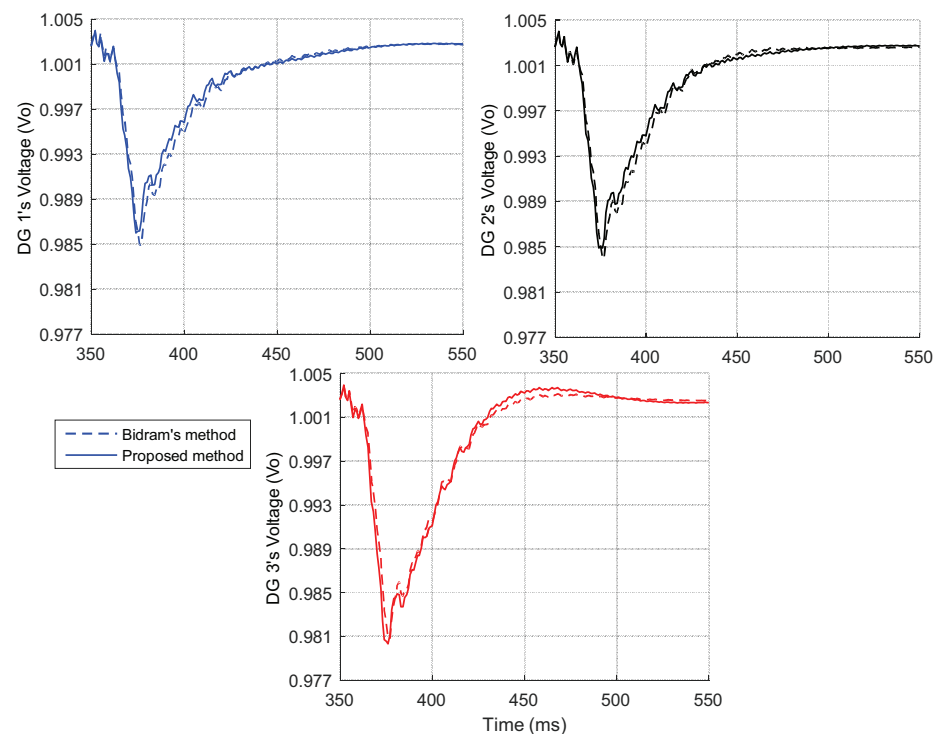

Fig. 3. 31. DG Voltage responses of the proposed hierarchical multi-agent and [38] method in case of DG outage

\section{B.2. Load change}

In Fig. 3.32, the DG voltage responses using both methods are compared for a load change applied to the system. As it can be seen in the simulation results of this case, in spite of slower multi-agent structure, the reference control input proposed by Bidram is more appropriate for voltage control purpose. For instance, the average overshoot in the responses of proposed method is increased by 7\% comparing with [39]. This fact is resulted from using improved input control signal in [39] which was discussed in (3.62).
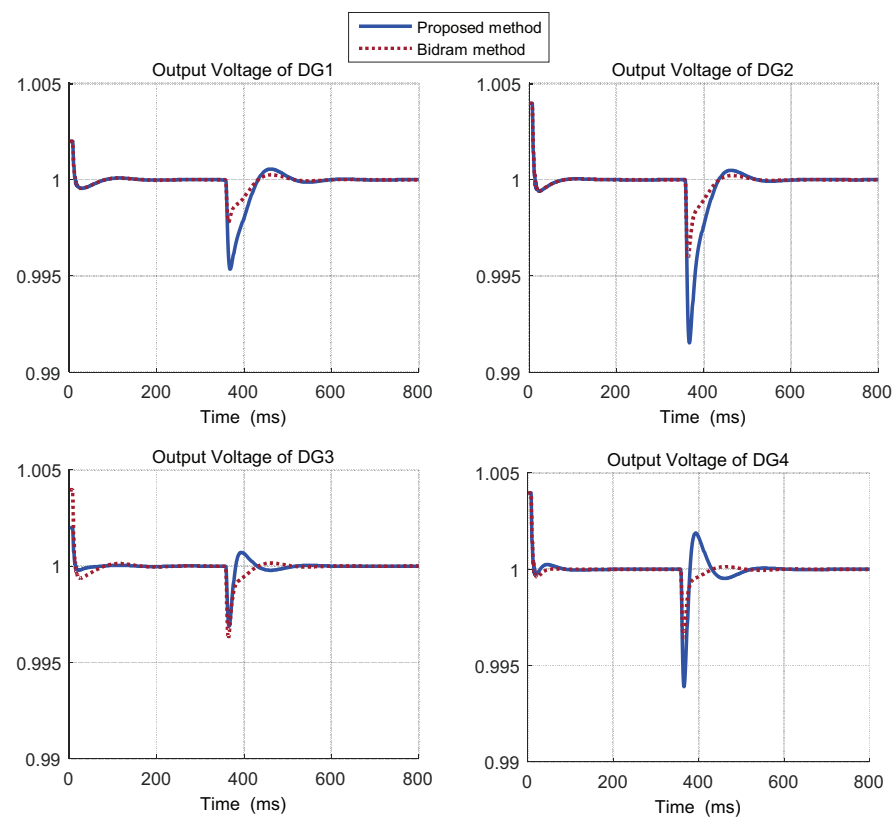

Fig. 3. 32. DG Voltage responses of the proposed hierarchical multi-agent and [38] method in case of load change

\section{V- CONCLUSIONS FOR CHAPTER 3}

This study was undertaken in order to analyze the application of optimal secondary control and multi-agent based control, which are proposed in this chapter for a smart microgrid [40]. The optimization algorithm that is used for tuning the control parameters to minimize oscillations is Particle Swarm Optimization (PSO), which is fast enough for transient analysis. ADP is also presented 


\section{KESHTKAR: MODELING AND CONTROLLING A HYBRID MULTI-AGENT BASED MICROGRID IN PRESENCE OF DIFFERENT PHYSICAL AND CYBER COMPONENTS}

and utilized in order to compare with the optimal controller. The linear dynamic modeling of the inverter based microgrid is discussed and utilized for small signal stability analysis purpose. Then nonlinear model of the microgrid is presented in order to investigate the effect of proposed hierarchical structure of multi-agent based control. First, the performances of the proposed control methods have been tested through simulations and observations. Then, the reliability and effectiveness of the proposed multi-agent structure have been evaluated with existing published works and analyzed against different perturbations in the system such as stochastic and DG outages. Simulation results show that the proposed control method is fast enough and acts appropriately and effectively when facing load changes. Also, the hierarchical multi-agent based control is proved to be more practical and effective than distributed and centralized structures.

\section{VI- REFERENCES FOR CHAPTER 3}

[1] S. Bhowmik, K. Tomsovic, and A. Bose, "Communication models for third party load frequency control," IEEE Trans. Power Syst., vol. 19, no. 1, pp. 543548, Feb. 2004.

[2] T. Bhatti and D. Kothari, "Variable structure LFC of isolated wind-diesel-microhydro hybrid power systems," J. Inst. Eng., vol. 83, pp. 52-56, 2002.

[3] S. Y.M. Atwa, E.F. El-Saadany, M.M.A. Salama, R. Seethapathy, " Optimal Renewable Resources Mix for Distribution System Energy Loss Minimization", IEEE Trans on Power Systems, vol. 25, no. 1, 2010.

[4] S. Mishra, G. Mallesham, and P. C. Sekhar, "Biogeography Based Optimal State Feedback Controller for Frequency Regulation of a Smart Microgrid ", IEEE Transaction on Smart Grid, vol. 4, no. 1, March 2013.

[5] I. J. Balaguer, Q. Lei, S. Yang, U. Supatti, and F. Z. Peng, "Control for grid-connected and intentional islanding operations of distributed power generation," IEEE Trans. Ind. Electron., vol. 58, no. 1, Jan. 2011.

[6] R.Majumder, B. Chaudhuri, A. Ghosh, R.Majumder, G. Ledwich, and F. Zare, "Improvement of stability and load sharing in an autonomousmicrogrid using supplementary droop control loop,” IEEE Trans. Power Syst., vol. 25, no. 2, pp. 796-808, May 2010.

[7] Z. Junxiong, L. Fei, C. Ze-xiang, Y. Jianzhong, "Coordinated Control Strategies between Unit and Grid in Islanded Power System", 4th International Conference on Electric Utility (DRPT), pp. 1454-1458, 2011.

[8] H. Bevrani, P. Ranjbar Daneshmand, "Fuzzy Logic-Based Load-Frequency Control Concerning High Penetration of Wind Turbines", IEEE System Journal, vol. 6, no. 1, pp. 173-180, March 2012.

[9] H. Bevrani, P. Ranjbar Daneshmand, P. Babahajyani, Y. Mitani, T. Hiyama, "Intelligent LFC Concerning High Penetration of Wind Power: Synthesis and Real-Time Application”, IEEE Transactions on Sustainable Energy, vol. 5, no. 2, pp. 655-662, April 2013.

[10] J. M. Guerrero, J. C. Vásquez, J. Matas, M. Castilla, L. G. d. Vicuña, and M. Castilla, "Hierarchical control of droop-controlled AC and DC microgrids-A general approach toward standardization," IEEE Trans. Ind. Electron., vol. 58, pp. 158-172, Jan. 2011.

[11] A. Bidram and A. Davoudi, "Hierarchical structure of microgrids control system," IEEE Trans. Smart Grid, vol. 3, pp. 1963-1976, Dec. 2012.

[12] M. D. Ilic and S. X. Liu, Hierarchical power systems control: Its value in a changing industry. London, U.K.: Springer, 1996.

[13] A. Mehrizi-Sani and R. Iravani, "Potential-function based control of a microgrid in islanded and grid-connected models," IEEE Trans. Power Syst., vol. 25, pp. 1883-1891, Nov. 2010.

[14] F. Katiraei, M. R. Iravani, and P. W. Lehn, "Microgrid autonomous operation during and subsequent to islanding process," IEEE Trans. Power Del., vol. 20, pp. 248-257, Jan. 2005.

[15] M. Savaghebi, A. Jalilian, J. Vasquez, and J. Guerrero, "Secondary control scheme for voltage unbalance compensation in an islanded droop-controlled microgrid,” IEEE Trans. Smart Grid, vol. 3, pp. 797- 807, June 2012.

[16] W. Gu, C. Shen, Z. Wu, "Multi-Agent Based Frequency Control of Islanded Microgrid," IREE, vol. 6, no. 7, pp. 3134-3141, Nov. 2011.

[17] Y. Xu, and W. Liu, "Novel Multi agent Based Load Restoration Algorithm for Microgrids," IEEE Trans. Smart Grid, vol. 2, no. I, pp. 152-161, Mar. 2011.

[18] BUSONIU L., BABUSKA R., DE SCHUTTER B.: 'A comprehensive survey of multi-agent reinforcement learning', IEEE Trans Syst. Man. Cybern. C: Appl. Rev., 2008, 38, (2), pp. 156-172.

[19] WEISS G. (ED.): 'Multi-agent systems: a modern approach to distributed artificial intelligence' (MIT Press, Cambridge, MA, 1999.

[20] W. Ren and R. W. Beard, "Consensus seeking in multi-agent systems under dynamically changing interaction topologies," IEEE Trans. Autom. Control, vol. 50, no. 5, pp. 655-661, May 2005. 
[21] W. Liu, Gu, Zhao, "A Noval Multi-Agent based Control Approach for Frequency Stabilization of Islanded Microgrids," 4th IEEE Innovative Smart Grid Technologies Europe (ISGT Europe), pp. 1-5, Oct 2013.

[22] D. E.rnst, "Reinforcement Learning applied to Power System Oscillations Damping", Proceedings of the 40th IEEE Conference on Decision and Control Orlando, Florida USA, December 2001.

[23] N. Pogaku, M. Prodanovic, T. Green, " Modeling, Analysis and Testing of Autonomous Operation of an Inverter-Based Microgrid", IEEE Transactions on Power Electronics, vol. 22, no. 2, pp. 613-625, March 2007.

[24] J. Lee, L. Wang "Small-Signal Stability Analysis of an Autonomous Hybrid Renewable Energy Power Generation/Energy Storage System Part I: TimeDomain Simulations", IEEE Transactions on Energy Conversion, vol. 23, no. 1, pp. 311-320, March 2008.

[25] Mohamed, Y.A.-R.I.; El-Saadany, E.F., "Adaptive Decentralized Droop Controller to Preserve Power Sharing Stability of Paralleled Inverters in Distributed Generation Microgrids," in Power Electronics, IEEE Transactions on, vol.23, no.6, pp.2806-2816, Nov. 2008

[26] Y.A.-R.I. Mohamed and E. F. El-Saadany "Hybrid variable-structure control with evolutionary optimum-tuning for fast grid-voltage regulation using inverter-based distributed generation", IEEE Trans. Power Electron., vol. 23, no. 3, pp.1334-1341 2008.

[27] F. Katiraei and M. R. Iravani "Power management strategies for a microgird with multiple distributed generation units", IEEE Trans. Power Syst., vol. 21, no. 4, pp.1821-18312006

[28] N. Pogaku, M. Prodanovic, and T. C. Green, "Modeling, analysis and testing of autonomous operation of an inverter-based microgrid," IEEE Trans. Power Electron., vol. 22, no. 2, pp. 613-625, Mar. 2007.

[29] M. N. Marwali and A. Keyhani, "Control of distributed generation systems part I: Voltage and currents control," IEEE Trans. Power Electron., vol. 19, no. 6, pp. 1541-1550, Nov. 2004.

[30] A. Keyhani, M. N. Marwali, and M. Dai, Integration of Green and Renewable Energy in Electric Power Systems. Hoboken, NJ, USA: Wiley, 2010.

[31] .Bidram, A.; Lewis, F.L.; Davoudi, A.; Zhihua Qu, "Frequency control of electric power microgrids using distributed cooperative control of multi-agent systems," in 2013 IEEE 3rd Annual International Conference on Cyber Technology in Automation, Control and Intelligent Systems (CYBER), , vol., no., pp.223-228, 26-29 May 2013

[32] G. Diaz, C. Gonzalez-Moran, J. Gomez-Aleixandre, and A. Diez, "Scheduling of droop coefficients for frequency and voltage regulation in isolated microgrids," IEEE Trans. Power Syst., vol. 25, no. 1, pp. 489-496, Feb. 2010.

[33] T.K. Das, G.K. Venayagamoorthy, "Optimal Design of Power System Stabilizers Using a Small Population Based PSO", PES General Meeting, IEEE, 2006.

[34] H. Keshtkar, J. Solanki, S.K. Solanki, "Application of PHEV in load frequency problem of a hybrid microgrid", North American Power Symposium (NAPS), 2012, IEEE, pp. 1-6.

[35] Gaurav. S. and Balakrishnan. S., "Adaptive critic based neurocontroller for autolanding of aircrafts", Proceedings of the American Control Conference, Vol 2, pp. 1081-1085, 1997.

[36] Ma. J., Yang. T., Hou. Z. G., "Dual Heuristic Programming Based Neurocontroller for Vibration Isolation Control", IEEE Proceeding of International Conference on Networking, Sensing and Control, Sanya, pp. 874 - 879, 2008.

[37] S. J Ahn, J. H. Choi, "Power Sharing and Frequency Control of an Autonomous Microgrid Considering the Dynamic Characteristics of Distributed Generations", Journal of International Council on Electrical Engineering, Volume 2, Issue 1, 2012

[38] A. Bidram, A. Davoudi, F.L Lewis, J.M. Guerrero, "Distributed Cooperative Secondary Control of Microgrids Using Feedback Linearization," in IEEE Transactions on Power Systems, vol.28, no.3, pp.3462-3470, Aug. 2013.

[39] A. Bidram, A. Davoudi, F. L. Lewis, "A Multiobjective Distributed Control Framework for Islanded AC Microgrids,", IEEE Transactions on Industrial Informatics, vol.10, no.3, pp.1785-1798, Aug. 2014.

[40] Some parts of this chapter have not been published yet and are copyrighted works.

\section{APPENDIX}

- State space matrices for each inverter model:

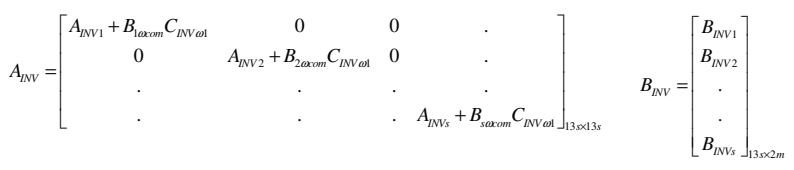

$C_{I N V}=\left[\begin{array}{cccc}{\left[C_{I N N c}\right]} & 0 & 0 & \cdot \\ 0 & {\left[C_{I N C 2}\right]} & 0 & \cdot \\ \cdot & \cdot & \cdot & \cdot \\ \cdot & \cdot & \cdot & {\left[C_{I N G s}\right]}\end{array}\right]_{2 \times \times 3 s}$ 
KESHTKAR: MODELING AND CONTROLLING A HYBRID MULTI-AGENT BASED MICROGRID IN PRESENCE OF DIFFERENT PHYSICAL AND CYBER COMPONENTS

- State space matrices for network model:

$A_{N E T}=\left[\begin{array}{cccc}A_{N E T 1} & 0 & 0 & \cdot \\ 0 & A_{N E T 2} & 0 & \cdot \\ \cdot & \cdot & \cdot & \cdot \\ \cdot & \cdot & \cdot & A_{N E T n}\end{array}\right]_{2 n \times 2 n} \quad B_{1 N E T}=\left[\begin{array}{c}B_{1 N E T_{1}} \\ B_{1 N E T_{2}} \\ \cdot \\ \cdot \\ B_{1 N E T_{n}}\end{array}\right]_{2 n \times 2 m} B_{2 N E T}=\left[\begin{array}{c}B_{2 N E T_{1}} \\ B_{2 N E T_{2}} \\ \cdot \\ \cdot \\ B_{2 N E T_{n}}\end{array}\right]_{2 n \times 1}$
$A_{N E T i}=\left[\begin{array}{cc}\frac{-r_{\text {linei }}}{L_{\text {linei }}} & \omega_{0} \\ -\omega_{0} & \frac{-r_{\text {linei }}}{L_{\text {linei }}}\end{array}\right] \quad B_{2 N E T i}=\left[\begin{array}{c}I_{\text {lineQ } i} \\ -I_{\text {lineDi }}\end{array}\right]_{B_{1 N E T i}}=\left[\begin{array}{ccccccc}\ldots & \frac{1}{L_{\text {linei }}} & 0 & \ldots & \frac{-1}{L_{\text {linei }}} & 0 & \ldots \\ \ldots & 0 & \frac{1}{L_{\text {linei }}} & \ldots & 0 & \frac{-1}{L_{\text {linei }}} & \ldots\end{array}\right]_{2 \times 2 m}$

- State matrices for load model:

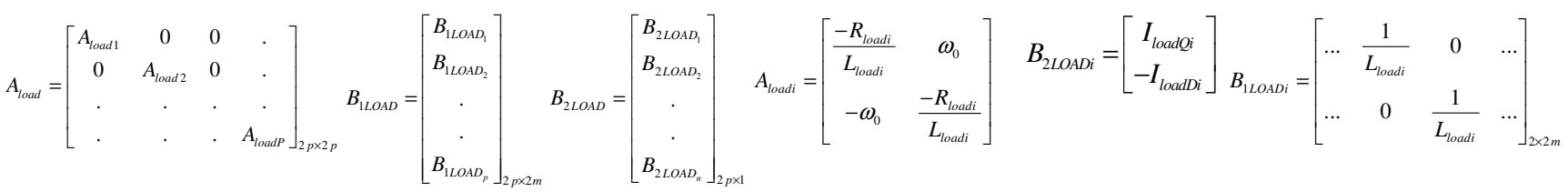

- State matrix values for DGs

$A_{D G}=\left[\begin{array}{ccccccccccccc}0 & -0.000094 & 0 & 0 & 0 & 0 & 0 & 0 & 0 & 0 & 0 & 0 & 0 \\ 0 & -31.41 & 0 & 0 & 0 & 0 & 0 & 0 & 0 & 358.074 & 12.564 & 11960 & 0 \\ 0 & 0 & -31.41 & 0 & 0 & 0 & 0 & 0 & 0 & 12.564 & -358.074 & 0 & 11960 \\ 0 & 0 & -0.0013 & 0 & 0 & 0 & 0 & 0 & 0 & -1 & 0 & 0 & 0 \\ 0 & 0 & 0 & 0 & 0 & 0 & 0 & 0 & 0 & 0 & -1 & 0 & 0 \\ 0 & 0 & -0.000065 & 390 & 0 & 0 & 0 & -1 & 0 & -0.05 & -0.0157 & 0.75 & 0 \\ 0 & 0 & 0 & 0 & 390 & 0 & 0 & 0 & -1 & 0.0157 & -0.05 & 0 & 0.75 \\ 0 & 0.000517 & -0.5055 & 3033333 & 0 & 11851851 & 0 & -7851 & 5.684 & -1129.6 & -122.1 & 5833 & 0 \\ 0 & 0.0010716 & 0 & 0 & 3033333 & 0 & 11851851 & -5.684 & -7851 & 122.1 & -1129.6 & 0 & 5833 \\ 0 & 0 & 0 & 0 & 0 & 0 & 0 & 20000 & 0 & 0 & 314 & -20000 & 0 \\ 0 & 0.0357952 & 0 & 0 & 0 & 0 & 0 & 0 & 20000 & -314 & 0 & 0 & -20000 \\ 17142.86 & -0.0000376 & 0 & 0 & 0 & 0 & 0 & 0 & 0 & 2857 & 0 & -85.714 & 314 \\ 1084286 & 0.0010716 & 0 & 0 & 0 & 0 & 0 & 0 & 0 & 0 & 2857 & -314 & -85.714\end{array}\right]_{13 s \times 13 s}$




\section{Chapter 4}

\section{CHALLENGES, CONCLUSIONS and FUTURE WORK}


KESHTKAR: MODELING AND CONTROLLING A HYBRID MULTI-AGENT BASED MICROGRID IN PRESENCE OF DIFFERENT PHYSICAL AND CYBER COMPONENTS

In this chapter challenges through providing this dissertation along with the conclusions obtained by simulation results and also recommended future works are discussed. One of the challenges during this dissertation was modeling the behavior of PHEV based on the existing stochastic and random processes. The related processes were selected based on the analysis on the pattern of different PHEV behavior, such as charging/discharging, and also the available references in this area. Another challenge was modeling the attack on the measurement signals in the microgrid control system. The consequences of different types of attacks have been modeled on control signals and worst case scenarios have been considered in designing the defense strategy. Considerable time was also spent on obtaining simulation results which have had power conditioning or optimization processes in control design such as microturbine model and secondary control for microgrid. Utilizing simplified models such as average model for inverter based DGs and linearized model for small signall stability analysis is one solution which is taken to reduce the simulation time. Also, proposing imroved mutation for optimization algorithms such as DE, has shown improvness in the speed of calculations. Communication delay was also a challenge in simulations in multi agent and cyber environments. This issue was analyzed and resolved in the last chapter by proposing new multi-agent structure.

Based on the first chapter of this dissertation, it is observed that accurate modeling of different component in a distibution power system may imrpove important aspects of the system. For example, there are different methods for approximating the integral terms in Carson's equations for calculating distribution line impedances and using these methods will result in change in power flow and short curcuit results. Hence, this variation in flows and voltages can lead to change in planning and designing aspects of protection devices such as fault clearing time of circuit breakers and impedance seen by distance relays. It is proved that the results of proposed method are closer to the exact values by numerical integration and Full Carson method than other approximate methods. Also, this method is easier to implement as it uses infinite series with less complicated functions. Similar conclusions are obtained from second part of this chapter which is related to modeling and optimal placing of DGs in a distribution power system. It shows that by optimal placing the DG systems such as PV in a distribution power system, the total daily loss will be minimized.

The distribution power system in the first chapter is equipped with small scale energy sources such as wind turbine generators, microturbines and plug-in hybrid electric vehicles to model the microgrid case study in the second chapter. Implementation of frequency control problem is proposed to mitigate the deviation of frequency due to the disturbances imposed to the microgrid such as load variations, PHEV stochastic behavior, and wind speed changes. A centralized multi agent system is implemented in the microgrid and different advanced control algorithms such as ADP, LQR, DE and PSO are utilized in the MGCC unit. Although MAS improves the effectiveness of the control algorithms, the existing communication infrastructure increases the potential of cyber attacks on the control signals. Therefore, a defense strategy through detaction and learning algorithm is proposed to be used by MGCC along with the controllers in order to regulate the frequency of the system affected by cyber intrusions. The simulation results show the resiliency of the system and the robustness of the proposed control and defense strategy against frequency oscillations due to cyber attacks with different timing and severity.

In the last chapter of the dissertation, the application of optimal secondary control and multi-agent based control in the modeled microgrid is investigated. The linear dynamic modeling of the inverter based microgrid is discussed and utilized for small signal stability analysis purpose. Based on the results, the appropriate states of the system can be identified in order to control and stabilize the microgrid system. Then nonlinear model of the microgrid is presented in order to investigate the effect of proposed hierarchical structure of multi-agent based control. Simulation results show the reliability and effectiveness of the proposed control method when facing voltage and frequency oscillations. Also, the proposed hierarchical structure is proved to be more practical 
and effective than distributed and centralized ones for the multi-agent based control due to the improved reliability and less delay existed in transmitting the control signals to MGCC unit in this structure.

Last discussion of this dissertation should be dedicated to the future plans for this work. Therefore, this part proposes the extensions that can be done on this work which take it to next level. Some of them could be a topic of new research plan.

- $\quad$ The microgrid which is modeled in this dissertation has different parts including Renewable Energy Sources (RES), such as Photovoltaic (PV) and Wind Turbine Generator (WTG), Energy Storage Systems (ESS) such as SMES, Distributed Generation (DG) resources such as Diesel Engine Generator (DEG), Fuel Cell (FC)/Aqua Electrolyzer (AE), and different type of demands such as Plug-in Hybrid Electric Vehicle (PHEV). Different simplifications have been applied in modeling of these devices. In fact, mostly the specifications which affect the transient response of the system are considered. The next step is to come up with more detailed modeling for the microgrid to analyze more realistic responses of the system.

- Modeling different parts of the distribution and micorogrid power system such as line impedances, PHEV, different DG units e.g. PV or MT system is investigated in this dissertation. This power system model can be utilized for applying important and critical analysis on the system. Further modeling for different generation and load units is recommended for future works on the modeled microgrid in order to increase the accuracy of the results and analysis.

- In order to improve the functionality of the multi-agent system a new layered multi-agent configuration can be designed to distinguish between normal and under-attack changes in the system and distribute the controller tasks in case of load and wind perturbation versus cyber intrusions. The accurate detection method plays a key role in this structure. This configuration will decrease the communication delay and improves the control aspects of the system against large frequency deviations.

- The communication infrastructure which is used in the multi-agent can be improved by using detailed communication protocols and also making interface with other software. Self-healing property is one of the useful aspects of the system against communication issue and can be studied for the modeled multi-agent system.

- $\quad$ The proposed Dynamic Heuristic Programming (DHP) method for frequency control is a new topic in the area of power system. Adding optimization and learning algorithm to the structure of DHP neural networks may result in better responses for frequency control purposes.

- In this study, cyber intrusions have been considered as a disturbance in the microgrid power system that deteriorates the frequency response of the system. In other words, the consequence of occurring a cyber attack is considered as case study for implementing control and defense strategy against disturbances in the modeled microgrid. Typically, having this model, impact analysis can be carried out for different attack scenarios to form the vulnerability index of the microgrid system. Modeling of the attack which can be applied for different system with different devices and the ability to extend the proposed method to systems with different topologies are the achievements that could be obtained by continuing this work.

- Practical implementation for the proposed hierarchical structure of multi-agent system in the last chapter is recommended in order to investigate the advantages of this method over centralized and distributed structures. The effect of delay in communication links can also be observed when the system is modeled with real agents. 